\title{
Studies of $\nu_{\mu} \rightarrow \nu_{e}$ Oscillation Appearance in the MINOS Experiment
}

\author{
A dissertation submitted by \\ Alexandre Bruno Pereira e Sousa \\ In partial fulfillment of the requirements \\ for the degree of \\ Doctor of Philosophy \\ in \\ Physics \\ TUFTS UNIVERSITY
}

February 2006

(c) 2006, Alexandre Sousa

Advisor: J. Schneps 


\section{Abstract}

The MINOS experiment uses a long baseline neutrino beam, measured $1 \mathrm{~km}$ downstream from its origin in the Near Detector at Fermilab, and $734 \mathrm{~km}$ later in the large underground Far Detector in the Soudan mine. By comparing these two measurements, MINOS can probe the atmospheric domain of the neutrino oscillation phenomenology with unprecedented precision. Besides the ability to perform a world leading determination of the $\Delta m_{23}^{2}$ and $\theta_{23}$ parameters, via $\nu_{\mu}$ flux disappearance, MINOS has the potential to make a leading measurement of $\nu_{\mu} \rightarrow \nu_{e}$ oscillations in the atmospheric sector by looking for $\nu_{e}$ appearance at the Far Detector. The observation of $\nu_{e}$ appearance, tantamount to establishing a non-zero value of the $\theta_{13}$ mixing angle, opens the way to studies of $C P$ violation in the leptonic sector, the neutrino spectral mass pattern ordering and neutrino oscillations in matter, the driving motivations of the next generation of neutrino experiments. In this thesis, we study the MINOS potential for measuring $\theta_{13}$ in the context of the MINOS Mock Data Challenge using a multivariate discriminant analysis method. We show the method's validity in the application to $\nu_{e}$ event classification and background identification, as well as in its ability to identify a $\nu_{e}$ signal in a Mock Data sample generated with undisclosed parameters. An independent shower reconstruction method based on three-dimensional hit matching and clustering was developed, providing several useful discriminator variables used in the multivariate analysis method. We also demonstrate that within 2 years of running, MINOS has the potential to improve the current best limit on $\theta_{13}$, from the CHOOZ experiment, by a factor of 2 . 
To my Parents... 


\section{Acknowledgments}

To Jay and Lucia, for unveiling new horizons of knowledge, kindness and generosity beyond any that I had previously encountered. You will be forever in my heart.

To Tony and Hugh, for their inspiring enthusiasm and love for physics and for painstakingly reviewing this work.

To the faculty members of the Physics Department, in particular Austin, Bill, Kris, Tom and Yaacov, for sharing their insightful knowledge of physics and making me feel at home while away from it.

To my fellow grad students and friends, Amir, Ang, Dan, Georgi, Hongwei, Karla, Vasilka, Vitaly, Xiangyan and Zlatko, for making these years at Tufts such an enjoyable and unforgettable experience.

To Jon and Howie, for their efficiency and professionalism in the construction of the batch processing framework and always being a pleasure to work with.

To Chris, Josh, Tingjun and Trish, fellow members of the $\nu_{e}$ appearance analysis group, for being such excellent people to work together with.

To my fellow MINOS colaborators Costas, Erik, Jeff, Simona and Rodolphe, and the E-towners Jen and Heather, for making the Northern Minnesota Winter feel so much warmer and for their continued friendship.

To Jennifer, Octavio and Victor, for many enjoyable moments together throughout these years in Boston.

To Helena, Nuno T., Nuno A. and Rui, fellow portuguese who chose an overseas path, for their constant support and friendship and many memorable moments.

To my friends in Portugal, Diogo, Filipe, Gonçalo, Kito, Né, Pedro e Tó-Zé for their perennial friendship and for always welcoming me back home so enthusiastically. 
To my undergraduate advisor, Ana Mourão, for her continued interest in my progress and unrelenting encouragement.

To the Ferreira Family, for welcoming me to Boston and treating me as one of their own.

To Massimo and Ornitorinko, for many wonderful evenings and stimulating conversations.

To Mayli, for being there every step of the way to rekindle glimmering spirits with hope, incentive and friendship. I will fondly remember our eclectic discussions over a good capuccino.

To my dear grandmother, for her loving care and tenderness and livening up my Boston home with her beautiful knittings.

To my wonderful sister and brother-in-law, for relentlessly encouraging me to pursue my dreams, and to little Alice, for being the most adorable niece.

To my parents, for their unconditional love and unwavering support. Although an ocean apart, you were there with me through all the good and not-so-good moments. You are the best parents a son could wish for.

This work was partially supported by the PRAXIS XXI grant BD-18360/98 from the Fundação para a Ciência e a Tecnologia, Lisbon, Portugal. 


\section{The MINOS Collaboration}

P. Adamson ${ }^{8,15}$, C. Andreopoulos ${ }^{21}$, R. Armstrong ${ }^{11}$, S. Avvakumov ${ }^{23}$, D.S. Ayres ${ }^{1}$, B. Baller ${ }^{8}$, B. Barish ${ }^{5}$, P.D. Barnes Jr. ${ }^{14}$, G. Barr ${ }^{18}$, W.L. Barrett ${ }^{30}$, E. Beall ${ }^{16}$, B.R. Becker ${ }^{16}$, A. Belias ${ }^{21}$, R. H. Bernstein ${ }^{8}$, D. Bhattacharya ${ }^{19}$, M. Bishai ${ }^{4}$, A. Blake ${ }^{6}$, B. Bock ${ }^{17}$, G.J. Bock ${ }^{8}$, J. Boehm ${ }^{9}$, D.J. Boehnlein ${ }^{8}$, D. Bogert ${ }^{8}$, P.M. Border ${ }^{16}$, C. Bower ${ }^{11}$, S. Boyd ${ }^{19}$, T. Brennan ${ }^{10}$, E. Buckley-Geer ${ }^{8}$, A. Cabrera ${ }^{18}$, D. Cherdack ${ }^{27}$, S.K. Chernichenko ${ }^{20}$, S. Childress ${ }^{8}$, B.C. Choudhary ${ }^{8}$, J.H. Cobb ${ }^{18}$, M. Dierckxsens ${ }^{4}$, M.V. Diwan ${ }^{4}$, M. Dorman ${ }^{15,21}$, D. Drakoulakos ${ }^{2}$, T. Durkin ${ }^{21}$, A.R. Erwin ${ }^{32}$, C.O. Escobar ${ }^{28}$, J. Evans ${ }^{18}$, E. Falk Harris ${ }^{24}$, G.J. Feldman ${ }^{9}$, T.H. Fields ${ }^{1}$, R. Ford ${ }^{8}$, M.V. Frohne ${ }^{3}$, H.R. Gallagher ${ }^{27}$, A. Godley ${ }^{22}$, J. Gogos ${ }^{16}$, M.C. Goodman ${ }^{1}$, P. Gouffon ${ }^{29}$, E. Grashorn ${ }^{17}$, N. Grossman ${ }^{8}$, K. Grzelak ${ }^{18}$, A. Habig ${ }^{17}$, D. Harris ${ }^{8}$, P.G. Harris ${ }^{24}$, J. Hartnell ${ }^{21}$, E.P. Hartouni ${ }^{14}$, R. Hatcher ${ }^{8}$, K. Heller ${ }^{16}$, C. Howcroft ${ }^{5}$, J. Hylen ${ }^{8}$, D. Indurthy ${ }^{26}$, G. Irwin ${ }^{23}$, M. Ishitsuka ${ }^{11}$, C. James ${ }^{8}$, L. Jenner ${ }^{15}$, D. Jensen ${ }^{8}$, T. Kafka ${ }^{27}$, S. Kahn ${ }^{4}$, H. Kang ${ }^{23}$, S.M.S. Kasahara ${ }^{16}$, G. Koizumi ${ }^{8}$, S. Kopp ${ }^{26}$, M. Kordosky ${ }^{15}$, D.J. Koskinen ${ }^{15}$, S.K. Kotelnikov ${ }^{13}$, A. Kreymer ${ }^{8}$, S. Kumaratunga ${ }^{16}$, K. Lang ${ }^{26}$, P.J. Litchfield ${ }^{16}$, R.P. Litchfield ${ }^{18}$, P. Lucas $^{8}$, E. Maher ${ }^{16}$, V. Makeev ${ }^{8}$, W.A. $\operatorname{Mann}^{27}$, A. Marchionni ${ }^{8}$, A.D. Marino ${ }^{8}$, M.L. Marshak ${ }^{16}$, J.R. Meier ${ }^{16}$, G.I. Merzon ${ }^{13}$, M.D. Messier ${ }^{11}$, D.G. Michael ${ }^{5 \dagger}$, W.H. Miller ${ }^{16}$, S.R. Mishra ${ }^{22}$, C. Moore ${ }^{8}$, J. Morfin ${ }^{8}$, L. Mualem ${ }^{16}$, S. Mufson ${ }^{11}$, S. Murgia ${ }^{23}$, J. Musser ${ }^{11}$, D. Naples ${ }^{19}$, C. Nelson ${ }^{8}$, J.K. Nelson ${ }^{31}$, H. Newman ${ }^{5}$, T.C. Nicholls ${ }^{21}$, J. Ochoa ${ }^{5}$, W. Oliver ${ }^{27}$, V.A. Onuchin ${ }^{20}$, T. Osiecki ${ }^{26}$, R. Ospanov ${ }^{26}$, J. Paley ${ }^{11}$, V. Paolone ${ }^{19}$, A. Para ${ }^{8}$, T. Patzak ${ }^{7}$, Z. Pavlovich ${ }^{26}$, G.F. Pearce ${ }^{21}$, C.W. Peck ${ }^{5}$, E.A. Peterson ${ }^{16}$, D.A. Petyt $^{16}$, H. Ping ${ }^{32}$, R. Piteira ${ }^{7}$, R.K Plunkett ${ }^{8}$, D. Rahman ${ }^{16}$, R.A. Rameika ${ }^{8}$, T. Raufer ${ }^{18}$, A.L. Read $^{8}$, B. Rebel ${ }^{8}$, J. Reichenbacher ${ }^{1}$, D.E. Reyna ${ }^{1}$, C. Rosenfeld ${ }^{22}$, H. Rubin ${ }^{10}$, K. Ruddick ${ }^{16}$, V.A. Ryabov ${ }^{13}$, R. Saakyan ${ }^{15}$, M.C. Sanchez ${ }^{9}$,

N. Saoulidou ${ }^{8}$, J. Schneps ${ }^{27}$, P. Schreiner ${ }^{3}$, V.K. Semenov ${ }^{20}$, P. Shanahan ${ }^{8}$, W. Smart ${ }^{8}$, V. Smirnitsky ${ }^{12}$, C. Smith ${ }^{24}$, A. Sousa ${ }^{27}$, B. Speakman ${ }^{16}$, P. Stamoulis ${ }^{2}$, P. Symes ${ }^{24}$, N. Tagg ${ }^{18}$, R.L. Talaga ${ }^{1}$, E. Tetteh-Lartey ${ }^{25}$, J. Thomas ${ }^{15}$,

M.A. Thomson ${ }^{6}$, I. Trostin ${ }^{12}$, V.A. Tsarev ${ }^{13}$, G. Tzanakos ${ }^{2}$, J. Urheim ${ }^{11}$, P. Vahle ${ }^{15}$, C. Velissaris ${ }^{32}$, V. Verebrysov ${ }^{12}$, B. Viren ${ }^{4}$, C.P. Ward $^{6}$, D.R. Ward ${ }^{6}$, M. Watabe ${ }^{25}$, A. Weber ${ }^{18,21}$, R.C. Webb ${ }^{25}$, A. Wehmann ${ }^{8}$, N. West ${ }^{18}$, C. White ${ }^{10}$, S.G. Wojcicki ${ }^{23 \dagger}$, D.M. Wright ${ }^{14}$, K. Wu ${ }^{22}$, T. Yang ${ }^{23}$, F.X. Yumiceva ${ }^{31}$, H. Zheng ${ }^{5}$, M. Zois ${ }^{2}$, and R. Zwaska ${ }^{26}$. 


\section{$\dagger$ Co-Spokespersons}

Argonne $^{1}$ - Athens $^{2}$ - Benedictine ${ }^{3}$ - Brookhaven ${ }^{4}$ - Caltech $^{5}$ Cambridge $^{6}$ - Collège de France ${ }^{7}$ - Fermilab ${ }^{8}$ - Harvard ${ }^{9}-$ IIT $^{10}$ Indiana $^{11}$ - ITEP-Moscow $^{12}$ - Lebedev ${ }^{13}$ - Livermore ${ }^{14}$ UCL-London $^{15}$ - Minnesota ${ }^{16}$ - Minnesota-Duluth ${ }^{17}$ - Oxford ${ }^{18}$ Pittsburgh $^{19}$ - Protvino ${ }^{20}$ - Rutherford ${ }^{21}$ - South Carolina ${ }^{22}$ Stanford $^{23}$ - Sussex $^{24}$ - Texas A\&M ${ }^{25}$ - Texas-Austin ${ }^{26}$ - Tufts $^{27}$ UNICAMP-Brazil $^{28}$ - USP-Brazil ${ }^{29}$ - Western Washington ${ }^{30}$ William \& Mary $^{31}$ - Wisconsin ${ }^{32}$ 


\section{Contents}

$\begin{array}{ll}\text { Introduction } & 1\end{array}$

1 Neutrino Physics 5

1.1 Neutrinos in the Standard Model . . . . . . . . . . . . . . . . 5

1.2 Neutrino Oscillations . . . . . . . . . . . . . . . . . . . 9

1.2.1 Three-Family Neutrino Mixing . . . . . . . . . . . . . 11

1.2.2 One Mass Scale Approximation . . . . . . . . . . . . . . . 13

1.2.3 CP Violation in the Leptonic Sector . . . . . . . . . . . . . . 14

1.3 Experimental Evidence of Neutrino Oscillations . . . . . . . . . . . 17

1.3.1 Solar Neutrinos . . . . . . . . . . . . . . . . . . . 17

1.3.2 Atmospheric Neutrinos . . . . . . . . . . . . . . . . . 21

1.3.3 Looking for $\theta_{13}$. . . . . . . . . . . . . . . . . . . . 25

1.3.4 Summary . . . . . . . . . . . . . . . 27

1.4 Future Neutrino Research . . . . . . . . . . . . . . . . . 27

2 The MINOS Experiment $\quad 31$

2.1 The NuMI Beam . . . . . . . . . . . . . . . . . . . . . . . . 32

2.2 The MINOS Detectors . . . . . . . . . . . . . . . . . 33

2.2.1 Detector Technology . . . . . . . . . . . . . . 34

2.2.2 The Far Detector . . . . . . . . . . . . . . . 36

2.2.3 The Near Detector . . . . . . . . . . . . . . . . . 38

2.2.4 Detector Calibration and Response . . . . . . . . . . . . . . . 40

2.3 MINOS Physics Reach . . . . . . . . . . . . . . 46

2.4 MINOS Atmospheric and Beam Neutrino Data . . . . . . . . . . 49 
2.5 MINOS Monte Carlo Data . . . . . . . . . . . . . . . . . 51

3 The Mock Data Challenge $\quad 55$

3.1 Event Samples and Generation _. . . . . . . . . . . . 56

3.2 Data Reduction . . . . . . . . . . . . . . . . . . . . 60

3.2 .1 Batch Processing . . . . . . . . . . . . . . 61

3.2 .2 Reconstruction . . . . . . . . . . . . . . . . . . 64

3.2 .3 Reconstruction ntuples . . . . . . . . . . . . . . 66

3.3 The NueAna framework . . . . . . . . . . . . . . . . . . . . 68

3.4 3D Hit Shower Reconstruction . . . . . . . . . . . . . . . . . . 69

3.4 .1 3D Hit Matching . . . . . . . . . . . . . . . 70

3.4 .2 Angular Clustering . . . . . . . . . . . . . . . . 74

3.4 .3 Shower Fitting . . . . . . . . . . . . . . 78

3.5 The Tufts Analysis Variables . . . . . . . . . . . . . . . . . 83

4 The $\nu_{\mu} \rightarrow \nu_{e}$ Appearance Oscillation Analysis $\quad 87$

4.1 Multivariate Discriminant Analysis . . . . . . . . . . . . 88

4.1.1 Principal Component Analysis . . . . . . . . . . . . . 88

4.1.2 The Fisher Discriminant Function . . . . . . . . . . . . . 88

4.1 .3 Mahalanobis Distance . . . . . . . . . . . . . . . . . 93

4.1.4 Classification Scores . . . . . . . . . . . . . . . 9 95

4.1.5 Stepwise Discriminant Analysis _ . . . . . . . . . . . . 96

4.1 .6 Implementation . . . . . . . . . . . . . . . . . . . . . 98

4.2 Background to $\nu_{\mu} \rightarrow \nu_{e}$ Appearance in MINOS $\ldots \ldots \ldots \ldots 9$

4.2 .1 Neutral Current Events . . . . . . . . . . . . . . . . . . . 101

4.2.2 Charged Current $\nu_{\mu}$ Events . . . . . . . . . . . . . . 101

4.2 .3 Charged Current $\nu_{\tau}$ Events . . . . . . . . . . . . . 103

4.2.4 Beam-induced Charged Current $\nu_{e}$ Events . . . . . . . . . 104

4.3 Analysis Samples . . . . . . . . . . . . . . . . . . . . . 105

4.4 Sample Cuts . . . . . . . . . . . . . . . . . . . . 107

4.5 Variable Selection . . . . . . . . . . . . . . . . . . . 111 
4.6 Analysis Procedure . . . . . . . . . . . . . . . . . . . . 113

4.6 .1 MDA Training . . . . . . . . . . . . . . . 114

4.6.2 MDA Threshold Determination . . . . . . . . . . . . 115

4.6.3 MDA Classification Results . . . . . . . . . . . . . . . . 119

4.6.4 MDA Performance . . . . . . . . . . . . . . . . . . . 121

5 Results and Discussion 131

5.1 Classification of the Near Sample . . . . . . . . . . . . . . 131

5.2 NEUGEN Systematics and MC Reweighting . . . . . . . . . . . 132

5.3 MINOS Sensitivity to $\nu_{e}$ Appearance . . . . . . . . . . . . . . 134

5.4 Mock Data Fitting Results . . . . . . . . . . . . . . . . . . . 135

5.5 MDC True Parameters . . . . . . . . . . . . . . . . . . . . 144

5.6 Concluding Remarks . . . . . . . . . . . . . . . . . . . . 145

$\begin{array}{ll}\text { A Mock Data Challenge Reco Scripts } & 147\end{array}$

A.1 Far Detector Monte Carlo Script . . . . . . . . . . . . . . 147

A.2 Far Detector Mock Data Script . . . . . . . . . . . . . . . . 151

A.3 Near Detector Monte Carlo Script . . . . . . . . . . . . . . . . 155

A.4 Near Detector Mock Data Script. . . . . . . . . . . . . 158

$\begin{array}{ll}\text { B AnaNue Variable Glossary } & 163\end{array}$ 


\section{List of Figures}

1.1 Neutrino scattering charged and neutral current Feynman diagrams . 8

1.2 Mass spectral patterns for three neutrinos . . . . . . . . . . . 13

1.3 Break down of the solar processes that produce neutrinos . . . . . . 18

1.4 Predicted SSM solar neutrino fluxes . . . . . . . . . . . . . . . 19

1.5 Comparison of solar neutrino experiment results with SSM . . . . . . 20

1.6 Solar neutrino oscillation contours . . . . . . . . . . . . . . 22

1.7 Early atmospheric neutrino results . . . . . . . . . . . . . 23

1.8 SuperK zenith angle distribution . . . . . . . . . . . . . . . . 24

1.9 Atmospheric neutrino oscillation contours . . . . . . . . . . 25

$1.10 \theta_{13}$ result summary . . . . . . . . . . . . . . 26

1.11 World summary for neutrino oscillation evidence . . . . . . . . . . . 28

1.12 The neutrino mass spectrum when experimental results are taken into

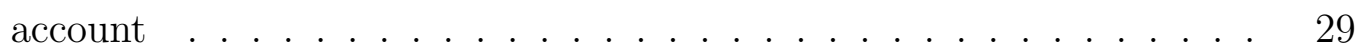

2.1 MINOS experiment overview . . . . . . . . . . . . . . . . 31

2.2 The layout of the NuMI beamline . . . . . . . . . . . . . . . 33

2.3 The NuMI beam spectra . . . . . . . . . . . . . . . . . 34

2.4 MINOS scintillator plane diagram . . . . . . . . . . . . . . 35

2.5 The MINOS scintillator strips and PMTs . . . . . . . . . . . . 36

2.6 The Far Detector . . . . . . . . . . . . . . . . . . . . . 37

2.7 The Near Detector . . . . . . . . . . . . . . . . . . . 40

2.8 ADC vs charge non-linearity for an electronics channel . . . . . . . . 41

2.9 Light injection system Diagram . . . . . . . . . . . . . . . 42

2.10 Light injection average channel gain vs time . . . . . . . . . . 43 
2.11 Effects of strip-to-strip calibration . . . . . . . . . . . 44

2.12 The Calibration Detector . . . . . . . . . . . . . . . 45

2.13 MINOS physics capabilities $-\nu_{\mu}$ spectral distortion measurement . . . 47

$2.14 \nu_{e}$ appearance sensitivity and $3 \sigma$ discovery contours $\ldots \ldots \ldots . . .48$

2.15 MINOS CPT violation allowed regions . . . . . . . . . . . 49

2.16 Cosmic ray muons $1 / \beta$ and zenith angle distributions $\ldots \ldots \ldots$

2.17 Evolution of MINOS proton intensity . . . . . . . . . . 51

3.1 Mock Data Challenge selection probability for $\nu_{\mu} \rightarrow \nu_{\tau}$ oscillation pa-

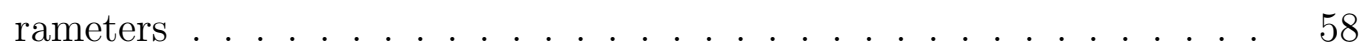

3.2 Mock Data Challenge selection probability for $\nu_{\mu} \rightarrow \nu_{e}$ and $\nu_{\mu} \rightarrow \nu_{\text {sterile }}$ oscillation parameters . . . . . . . . . . . . . . . . . 58

3.3 MINOS Batch Processing diagram . . . . . . . . . . . . 63

3.4 Daily Summary of CPU usage at the Batch Processing Farm . . . . . 64

3.5 NueAna framework structure . . . . . . . . . . . . . . . 70

3.6 The MINOS coordinate systems . . . . . . . . . . . . . . . . 72

3.7 3D hit matching algorithm . . . . . . . . . . . . . . 73

3.8 3D hit matching example application . . . . . . . . . . 74

3.9 Spherical coordinate representations of an event $\ldots \ldots \ldots \ldots$

3.10 Angular clustering algorithm summary . . . . . . . . . . . 77

3.11 Clustering and fitting example . . . . . . . . . . . . . 83

3.12 Tufts variable distributions . . . . . . . . . . . . . . . 85

4.1 Principal component analysis illustration . . . . . . . . . . . . . 89

4.2 Motivation for the introduction of Fisher's linear discriminant . . . . 89

4.3 Comparison between PCA and FLD . . . . . . . . . . . 90

$4.4 \quad$ FLD application example . . . . . . . . . . . . . . . . . . . 92

4.5 Loci of points at equal euclidian and standard distances . . . . . . . 94

4.6 Loci of points at equal Mahalanobis distance . . . . . . . . . . 95

4.7 DISCRIM procedure classifcation output example . . . . . . . . 100

4.8 An example of a background $\mathrm{NC}$ event . . . . . . . . . . . . . 101

4.9 An example of a background $\mathrm{CC} \nu_{\mu}$ event $\ldots \ldots \ldots \ldots$ 
4.10 An example of a background CC $\nu_{\tau}$ event . . . . . . . . . . . . 103

4.11 Expected flux of the $\nu_{e}$ component of the NuMI beam at the Far Detector 104

4.12 Energy spectrum comparison between signal and beam $\nu_{e}$ events . . . 105

4.13 An example of a background beam-induced CC $\nu_{e}$ event . . . . . . . 106

4.14 Example of harmonization of Near and Far variable distributions by a sample cut . . . . . . . . . . . . . . . . . . 109

4.15 Example of SAS STEPDISC variable selection output . . . . . . . . . 112

4.16 Classification scores divided by class of events (logarithmic scale) . . 116

4.17 Classification scores divided by class of events (normalized to the signal $\nu_{e}$ histogram) . . . . . . . . . . . . . . . . . 117

4.18 Event probability of belonging to the signal $\nu_{e}$ group against the other probabilities ........................ 118

4.19 Probability threshold determination . . . . . . . . . . . . . 118

4.20 MDA ROC curve . . . . . . . . . . . . . . . . . . . . . . 119

4.21 SAS STEPDISC variable selection output for the best MDA classifier 121

4.22 FOM vs. Probability Threshold for MDA's best classifier . . . . . . . 122

4.23 MDA efficiency and purity as a function of the probability threshold cut123

4.24 MDA efficiency and purity as a function of the reconstructed event energy . . . . . . . . . . . . . . . . . . . . 123

4.25 Visible energy distribution of MDA classified events . . . . . . . . . . 124

4.26 Excess $\nu_{e}$ appearance signal over expected background . . . . . . . 125

4.27 MDA true resonance code distribution . . . . . . . . . . . . 125

4.28 MDA true EM shower energy fraction and $y$ distributions . . . . . . . 126

4.29 MDA true neutrino energy and event length distributions . . . . . . . 126

4.30 Example of NC background event with low electromagnetic energy fraction . . . . . . . . . . . . . . . . . . 128

4.31 Example of NC background event with low visible energy . . . . . . . 128

4.32 Example of a $\mathrm{CC} \nu_{\mu}$ background event with low electromagnetic energy fraction . . . . . . . . . . . . . . . . . . . 129

5.1 MDA sensitivity to $\nu_{\mu} \rightarrow \nu_{e}$ appearance for two exposures $\ldots \ldots$. . 136 xvii 
5.2 Evolution of $\chi^{2}$ value with the variation of Mock Data fit parameters 138

5.3 MDC fit $\chi^{2}$ curves for two exposures . . . . . . . . . . . . . . 139

5.4 MDC fit $\Delta \chi^{2}$ curves for two exposures . . . . . . . . . . . . . . . . . 140

5.5 MDC fit visible energy spectra for two exposures . . . . . . . . . . . . 141

5.6 MDC fit 1D confidence intervals for two exposures . . . . . . . . . 143

$5.7 \nu_{\mu} \rightarrow \nu_{e}$ analysis summary . . . . . . . . . . . . 145 


\section{List of Tables}

1.1 Properties of bosons $\ldots \ldots \ldots \ldots \ldots \ldots \ldots$

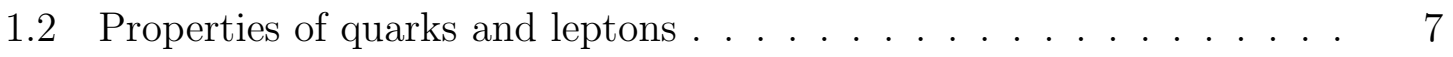

3.1 Break down of the Mock Data Challenge sample . . . . . . . . 57

3.2 Tabulated and calculated radiation length and Molière radius for relevant materials. . . . . . . . . . . . . . . . . 82

4.1 Summary of the composition of the analysis samples . . . . . . . 107

4.2 Cut summary table for the Far Test sample . . . . . . . . . . . 110

4.3 MDA classification summary table (SuperK osc., $9.25 \times 10^{20}$ POT exposure weights $\ldots \ldots \ldots \ldots \ldots$

4.4 MDA classification summary table (MDC osc., $7.4 \times 10^{20}$ POT exposure weights $\ldots \ldots \ldots \ldots \ldots \ldots \ldots$

5.1 MDA Near MC sample classification summary . . . . . . . . . . 132

5.2 NEUGEN parameters default values and nominal weights . . . . . . 134

5.3 MDA classification summary for the Near and Far Mock Data samples 136

5.4 Mock Data nominal values of fit parameters . . . . . . . . . 137

5.5 MDC best fit parameter values . . . . . . . . . . . . . . 142

5.6 MDC truth values . . . . . . . . . . . . . . . . . . . . . . . . . 144 
XX 


\section{Introduction}

Since it was first proposed by Pauli in 1930, in his famous letter [1] to the Group on Radioactivity gathered for a regional meeting in Tübingen, the neutrino has continued to bewilder the physics community with its unexpected properties.

Originally called "neutron", and proposed as a desperate remedy to prevent the collapse of the principle of conservation of energy, the particle Fermi renamed as "neutrino" was only experimentally observed in 1953, by Cowan and Reines [2].

In 1956-1958, with a remarkable experiment connecting the helicity ${ }^{1}$ of the neutrino with the observable helicity of the photon emitted in the decay of an excited state of the isotope ${ }^{152} \mathrm{Sm}$, Goldhaber, Grodzins, and Sunyar showed that all observed neutrinos display negative helicity [3].

In 1961-1962, Lederman, Schwartz, Steinberger and others used the Alternating Gradient Synchrotron (AGS) at Brookhaven National Laboratory to find evidence of a new flavor of neutrino, the muon neutrino [4]. A beam of the AGS's energetic protons would produce a shower of pi mesons, which decay into muons and neutrinos. Only the neutrinos could go through a 5 kton steel wall and be detected in a spark chamber. The interactions observed originated from muon neutrinos. A third neutrino flavor, the tau neutrino was detected only in 2000 by the DONUT experiment [5]. The DONUT collaboration includes several members of the Tufts High Energy Physics Group.

Following the unexpected discrepancies between the predicted flux of neutrinos emanating from the Sun and the flux observed in several radio-chemical experiments

\footnotetext{
${ }^{1}$ Helicity of a particle is defined as the projection of its spin vector in the direction of its momentum. A particle with negative helicity is also called left-handed, as the particle will "spin" in the same way as the curling fingers of the left hand when the thumb points along the direction of motion of the particle.
} 
during the 70s, 80s and 90s, several explanations were considered, with the most popular one based on the idea that neutrinos can undergo flavor change in a process now referred to as neutrino oscillations. This idea is an evolution of Pontecorvo's [6] 1957 suggestion of the possibility of neutrino-anti-neutrino oscillations.

In recent years, the Super-Kamiokande experiment gathered strong evidence that atmospheric neutrinos, produced by cosmic rays, do oscillate [7], a claim that has been independently verified by the Soudan 2 experiment [8]. Furthermore, in combination with the Super-Kamiokande solar neutrino data, the SNO experiment found evidence that the neutrino oscillation mechanism correctly accounts for the solar neutrino anomaly [9]. The possibility that neutrinos oscillate implies they must have a mass, albeit very small. Since neutrinos are described by the very successful Standard Model of Fundamental Particles and Interactions as massless particles, the confirmation of neutrino oscillations offers the first experimental evidence of physics beyond the Standard Model since its emergence in the 1970's.

The parameters determining the neutrino oscillatory behavior in the "atmospheric" range will be measured with unprecedented precision by the MINOS experiment, the main focus of this thesis and the first of a new generation of long baseline experiments that marks the onset of a "precision era" in experimental neutrino physics. MINOS will be able to probe the tantalizing possibility that $\nu_{\mu} \rightarrow \nu_{e}$ oscillations occur in the "atmospheric" domain by looking for appearance of electron neutrino events from a neutrino beam almost exclusively composed of muon neutrinos. The observation of $\nu_{\mu} \rightarrow \nu_{e}$ oscillations is the only known process which may probe the existence of $C P$ violation in the leptonic sector, a possibility that opens new avenues in the explanation of the matter-antimatter baryon asymmetry and has now become the driving motivation behind all of the next generation neutrino experiments, such as NOvA and $\mathrm{T} 2 \mathrm{~K}$.

The goal of the research described in this thesis consists in the exploration of the potential of the MINOS experiment to observe $\nu_{\mu} \rightarrow \nu_{e}$ oscillations. To do so, an independent shower reconstruction technique was developed and a classification mechanism based on a multivariate discriminant method was implemented in the context of the MINOS Mock Data Challenge. 
In the first Chapter of the thesis, the aspects of neutrino physics mentioned above are described in detail and the experimental evidence for neutrino oscillations is outlined. A detailed characterization of the MINOS experiment ensues in the second chapter, followed by a discussion of the aspects involved in the MINOS Mock Data Challenge implementation, as well as of the development of a cluster algorithm for shower reconstruction based on 3D Hits. The fourth chapter delineates the multivariate discriminant method used in event classification and provides an account of the analysis procedure employed, in conjunction with the achieved classification results. In the last chapter, the analysis method is applied to the challenge sample to test the existence of a $\nu_{\mu} \rightarrow \nu_{e}$ oscillation signal. The final results thus obtained are presented, followed by a concluding discussion. 


\section{Chapter 1}

\section{Neutrino Physics}

In this Chapter, a description of the neutrinos as fundamental particles of the Standard Model is given, followed by a description of neutrino oscillation phenomenology, with some focus on $\nu_{\mu} \rightarrow \nu_{e}$ oscillations and $C P$ violation in the leptonic sector. The existing experimental evidence for neutrino oscillations is summarized and in conclusion, a discussion of future neutrino experiments is presented.

\subsection{Neutrinos in the Standard Model}

Pauli's proposal of the neutrino was a solution to the observed continuous spectrum of energies in $\beta$ decay of radioactive isotopes, which appeared to violate energy conservation:

$$
{ }_{Z}^{A} X \rightarrow{ }_{Z+1}^{A} Y+e^{-} .
$$

In 1934, Fermi's theory of $\beta$ decay [10] incorporated Pauli's idea and explained the phenomenon as a process where one of the nuclear constituents, a neutron, decays into a proton, the observed electron and the "little neutral one", the neutrino. More rigorously:

$$
n \rightarrow p+e^{-}+\bar{\nu}_{e} .
$$

The theory underwent many improvements until 1956, when parity violation in $\beta$ decay was suggested by Lee and Yang [11] and confirmed experimentally by $\mathrm{Wu}$ et al. [12] in 1957. To account for this, Fermi's theory was modified by Marshak and Sudarshan and by Feynman and Gell-Mann into the V-A (Vector-Axial-Vector) 
theory of weak interactions $[13,14]$, which introduced the weak lagrangian:

$$
\mathcal{L}_{W}=\frac{G_{F}}{\sqrt{2}} J_{W}^{\mu} J_{\mu W}^{\dagger}
$$

where $G_{F} \approx 1.17 \times 10^{-5} \mathrm{GeV}^{-2}$ is the Fermi coupling and $J_{W}^{\mu}=J_{\text {lepton }}^{\mu}+J_{\text {hadron }}^{\mu}$ is the weak current. The parity violating leptonic current is given by:

$$
J_{\text {lepton }}^{\mu}=\bar{\psi}_{l} \gamma^{\mu}\left(1-\gamma^{5}\right) \psi_{\nu_{l}}
$$

where $\psi_{l}, \psi_{\nu_{l}}$ are the charged lepton field and its associated neutrino field, and $\left(1-\gamma^{5}\right)$ represents the parity violation (i.e. the helicities of the initial and final states of an interaction are different). The Goldhaber et al. measurement of the helicity of the neutrino and the fact that the theory also provided a natural explanation for the rarity of pion decay into electrons were fundamental in establishing the $\mathrm{V}$-A nature of weak interactions.

The V-A theory of weak interactions was unified with the theory of quantum electrodynamics [15] by the Glashow-Weinberg-Salam (GWS) model [16, 17, 18], which in combination with the theory of quantum chromodynamics (QCD) [19, 20, 21], forms the Standard Model of Fundamental Particles and Interactions. The Standard Model is an $S U(3)_{C} \otimes S U(2)_{L} \otimes U(1)_{Y}$ gauge theory. The fundamental particles in the Standard Model consist of the integer spin interaction mediating bosons, which follow Bose-Einstein statistics, and three families of spin $\pm 1 / 2$ leptons and quarks that obey Fermi-Dirac statistics. The properties of the Standard Model bosons are shown in Table 1.1 [22].

The properties of leptons and quarks are summarized in Table 1.2 [22].

The neutrinos can couple to the weak force carrier bosons through charged current (CC) and neutral current (NC) processes, as shown in Fig. 1.1.

In the Standard Model, the mass of the fermions results from the coupling of the left-hand doublet and the right-hand singlet fermion fields with the Higgs scalar field. The mass terms in the lagrangian are only non-zero if the left-hand and right-hand fields are simultaneously present. However, unlike the charged leptons, the neutrino does not have a right-hand singlet in the Standard Model, so it has no coupling to the Higgs field and is therefore massless. 


\begin{tabular}{|c|c|c|c|c|}
\hline Boson & $Q$ & Spin & Mass $(\mathrm{GeV})$ & Interaction \\
\hline \hline$\gamma$ & 0 & 1 & 0 & Electromagnetic \\
\hline \hline$W^{ \pm}$ & \pm 1 & 1 & 80 & Weak \\
\hline$Z^{0}$ & 0 & 1 & 91 & Weak \\
\hline \hline gluon (8) & 0 & 1 & 0 & Strong \\
\hline \hline H (Higgs) & 0 & 0 & $>115$ & - \\
\hline
\end{tabular}

Table 1.1: Properties of bosons in the Standard Model. The electromagnetic charge $Q$, spin, mass and interaction mediated is displayed for each boson. The gluons (carriers of color, which is the QCD symmetry) can occur in 8 color-anticolor combinations of "red", "green" and "blue". The Higgs boson couples every massive particle with the Higgs field. The Higgs field is not considered a force as it does not accelerate particles and it does not transfer energy. It confers a mass to a particle proportional to that particle's coupling strength with the field.

\begin{tabular}{|c|c|c|c|c|}
\hline Flavor & $Q$ & $\left(I_{3}\right)_{L}$ & $Y$ & Mass $(\mathrm{MeV})$ \\
\hline \hline$e$ & -1 & $-\frac{1}{2}$ & -1 & 0.511 \\
\hline$\nu_{e}$ & 0 & $+\frac{1}{2}$ & -1 & $<3 \times 10^{-6}$ \\
\hline \hline$\mu$ & -1 & $-\frac{1}{2}$ & -1 & 105.7 \\
\hline$\nu_{\mu}$ & 0 & $+\frac{1}{2}$ & -1 & $<0.19$ \\
\hline \hline$\tau$ & -1 & $-\frac{1}{2}$ & -1 & 1777 \\
\hline$\nu_{\tau}$ & 0 & $+\frac{1}{2}$ & -1 & $<18.2$ \\
\hline \hline $\mathrm{u}$ & $+\frac{2}{3}$ & $+\frac{1}{2}$ & $+\frac{1}{3}$ & $1.5-5$ \\
\hline $\mathrm{d}$ & $-\frac{1}{3}$ & $-\frac{1}{2}$ & $+\frac{1}{3}$ & $17-27$ \\
\hline \hline $\mathrm{c}$ & $+\frac{2}{3}$ & $+\frac{1}{2}$ & $+\frac{1}{3}$ & $1100-1400$ \\
\hline $\mathrm{s}$ & $-\frac{1}{3}$ & $-\frac{1}{2}$ & $+\frac{1}{3}$ & $60-170$ \\
\hline \hline $\mathrm{t}$ & $+\frac{2}{3}$ & $+\frac{1}{2}$ & $+\frac{1}{3}$ & 173800 \\
\hline $\mathrm{b}$ & $-\frac{1}{3}$ & $-\frac{1}{2}$ & $+\frac{1}{3}$ & $4100-4400$ \\
\hline
\end{tabular}

Table 1.2: Properties of leptons (upper half) and quarks (bottom half) in the Standard Model. $Q$ is the electromagnetic charge, $I_{3}$ is the weak isospin, with the $L$ subscript denoting that only left-handed particles or right-handed anti-particles feel the weak force, and $Y$ is the hypercharge of a particle The equivalent quantum numbers for the anti-particles are obtained by applying a multiplicative factor of -1 .

There is no fundamental symmetry in the Standard Model that predicts the neutrino mass to be zero, but an extension is necessary to accommodate massive neutrinos. That extension can be achieved by introducing an $S U(2) \operatorname{singlet} \nu_{R}$. This right-handed neutrino has no couplings with the weak force carrier bosons and is 

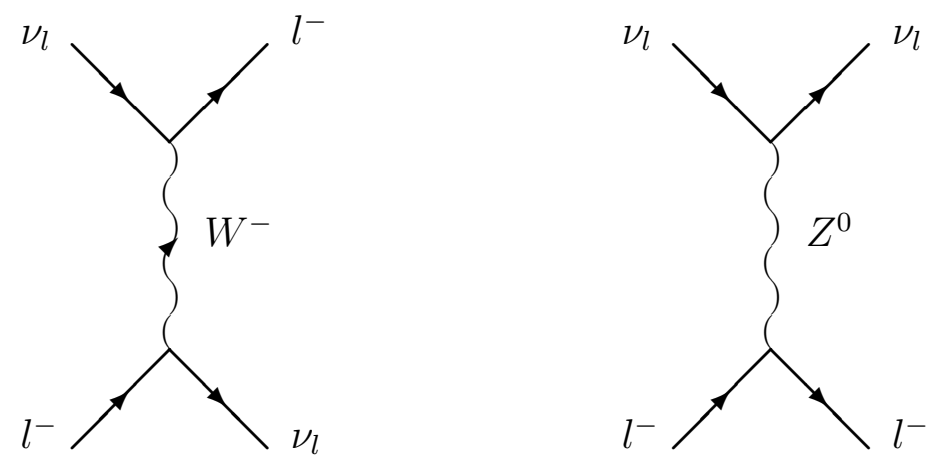

Figure 1.1: Feynman diagram representation of the charged current (left) and neutral current (right) contributions to the neutrino scattering process $\nu_{l}+l \rightarrow \nu_{l}+l$, where $l$ represents one of the three lepton families.

therefore "sterile". In this scenario, the neutrino mass can appear in two ways, as a Dirac mass term in the lagrangian, similar to the one for the charged leptons, or by introducing a Majorana mass term. The Dirac term assumes that a particle and its anti-particle are different, so that the right-handed state $\nu_{R}$ is different from $\nu_{R}^{c}$, the $C P T$ partner of the left-handed $\nu_{L}$. The Dirac mass term connecting the left and right-hand components of the same field is then:

$$
\mathcal{L}_{D}=-m_{D} \bar{\psi}_{\nu} \psi_{\nu}=m_{D}\left(\bar{\psi}_{\nu_{L}} \psi_{\nu_{R}}+\bar{\psi}_{\nu_{R}} \psi_{\nu_{L}}\right)
$$

where $\psi_{\nu}=\psi_{\nu_{R}}+\psi_{\nu_{L}}$. The Dirac mass term connects states of opposite helicity or chirality. Dirac neutrinos conserve lepton number and have four components, $\nu_{L}, \bar{\nu}_{R}$, $\nu_{R}, \bar{\nu}_{L}$, in identical fashion to the charged leptons.

In the case of Majorana neutrinos, we can construct a mass term using $\nu_{R}$ and its charge conjugate $\nu_{R}^{c}$, with $\psi^{c}=C(\bar{\psi})^{T}$ and the charge conjugation operator given by $C=i \gamma^{2} \gamma^{0}$. Because neutrinos are neutral, a neutrino can be its own anti-particle so that the CPT partner of $\nu_{L}$ is identical to the right-handed neutrino $\left(\nu_{L}\right)^{c}=\nu_{R}^{c}$. The Majorana mass term in the lagrangian is:

$$
\begin{aligned}
\mathcal{L}_{M} & =-m_{M} \bar{\psi}_{M} \psi_{M} \\
& =-m_{M}\left(\bar{\psi}_{\nu_{L}} \psi_{\nu_{R}}^{c}+\overline{\psi_{\nu_{R}}^{c}} \psi_{\nu_{L}}\right) \\
& =-m_{M}\left(\bar{\psi}_{\nu_{L}} C\left(\overline{\psi_{\nu_{L}}}\right)^{T}+C\left(\psi_{\nu_{L}}^{c}\right)^{T} \psi_{\nu_{L}}\right),
\end{aligned}
$$

where $\psi_{M}$ is a self-conjugate field such that $\psi_{M}=\psi_{\nu_{L}}+\psi_{\nu_{R}}^{c}=\psi_{M}^{c}$. An analogous mass term exists for $\nu_{R}$. The Majorana mass term connects neutrino states with 
the same chirality. When Majorana mass terms are present, lepton number is not conserved, so there is no quantum number left to distinguish a neutrino from its anti-particle and we have, for a given helicity $h, \overline{\nu_{i}}(h)=\nu_{i}(h)$. If the neutrino is a Majorana particle, neutrinoless double beta decay processes can occur. Experimental searches for these processes place an upper limit of $0.6 \mathrm{eV}$ on the mass of the electron neutrino [23].

The neutrino oscillation phenomenon offers an indirect method of probing neutrino mass, as it can provide a measure of the difference between squared mass values of the neutrino species. In the following Section, the neutrino oscillation phenomenology is reviewed.

\subsection{Neutrino Oscillations}

Neutrinos are created and observed in nature as eigenstates of the weak interaction $\left|\nu_{\alpha}\right\rangle$, where $(\alpha=e, \mu, \tau)$ corresponding to each charged lepton. Pontecorvo's idea assumes the weak eigenstates are not identical to the mass eigenstates, but are instead a linear superposition of them. Consider, for the moment, an arbitrary number $n$ of flavor states $\left|\nu_{\alpha}\right\rangle$ represented as linear superpositions of $n$ mass states $\left|\nu_{1}\right\rangle \ldots\left|\nu_{n}\right\rangle$ with masses $m_{1} \ldots m_{n}$. Disregarding time evolution, we have:

$$
\left|\nu_{\alpha}\right\rangle=\sum_{i=1}^{n} U_{\alpha i}^{*}\left|\nu_{i}\right\rangle,
$$

where $U$ is the PMNS (Pontecorvo-Maki-Nakagawa-Sakata) unitary leptonic mixing matrix of the transformation [24]. The time evolution of the state $\left|\nu_{\alpha}\right\rangle$ can be found by applying Schrödinger's equation to the $\nu_{i}$ component of $\nu_{\alpha}$ in the rest frame of that component:

$$
\left|\nu_{i}\left(\tau_{i}\right)\right\rangle=e^{-i m_{i} \tau_{i}}\left|\nu_{i}(0)\right\rangle,
$$

with $m_{i}$ the mass of $\nu_{i}$ and $\tau_{i}$ the time in the $\nu_{i}$ frame. Rewriting the Lorentz-invariant phase factor in Eq. 1.4 in terms of the time $t$ and position $L$ in the laboratory frame, we obtain:

$$
e^{-i m_{i} \tau_{i}}=e^{-i\left(E_{i} t-p_{i} L\right)},
$$


where $E_{i}$ is the energy and $p_{i}$ the momentum of $\nu_{i}$. If we now assume the $\nu_{\alpha}$ was produced with momentum $p$, shared by all of its mass eigenstate components, and furthermore that $p \gg m_{i}$, then the $\nu_{i}$ component has $E_{i}=\sqrt{p^{2}+m_{i}^{2}} \approx p+m_{i}^{2} / 2 p$. Moreover, the neutrinos are ultra-relativistic, so we consider $t \approx L$. The phase factor of Eq. 1.5 becomes thus

$$
e^{-i\left(m_{i}^{2} / 2 p\right) L}
$$

To find the evolution of state $\nu_{\alpha}$ after it propagated a distance $L$, we insert the latter result in Eq. 1.11 to find

$$
\left|\nu_{\alpha}(L)\right\rangle \approx \sum_{i=1}^{n} U_{\alpha i}^{*} e^{-i\left(m_{i}^{2} / 2 E\right) L}\left|\nu_{i}\right\rangle
$$

where $E \simeq p$ is the average energy of the mass eigenstate components of the neutrino. Using the unitarity of $U$, we can invert Eq. 1.11 and plug the result in Eq. 1.7:

$$
\left|\nu_{\alpha}(L)\right\rangle \approx \sum_{\beta=1}^{n}\left[\sum_{i=1}^{n} U_{\alpha i}^{*} e^{-i\left(m_{i}^{2} / 2 E\right) L} U_{\beta i}\right]\left|\nu_{\beta}\right\rangle
$$

After traveling a distance $L$, the $\nu_{\alpha}$ has become a superposition of all the flavors. The probability of measuring the $\nu_{\alpha}$ as a $\nu_{\beta}$ is

$$
\begin{aligned}
P\left(\nu_{\alpha} \rightarrow \nu_{\beta}\right) & =\left|\left\langle\nu_{\beta} \mid \nu_{\alpha}(L)\right\rangle\right|^{2} \\
& =\sum_{i, j} U_{\alpha i}^{*} U_{\beta i} U_{\alpha j} U_{\beta j}^{*} e^{-i \Delta m_{i j}^{2} L / 2 E} .
\end{aligned}
$$

Here, we used $\Delta m_{i j}^{2}=m_{i}^{2}-m_{j}^{2}$. If we now make the real and imaginary components explicit, we can recast Eq. 1.9 in the form:

$$
\begin{aligned}
P\left(\nu_{\alpha} \rightarrow \nu_{\beta}\right)=\delta_{\alpha \beta} & -4 \sum_{i>j} \Re\left(U_{\alpha i}^{*} U_{\beta i} U_{\alpha j} U_{\beta j}^{*}\right) \sin ^{2}\left(\frac{\Delta m_{i j}^{2} L}{4 E}\right) \\
& +2 \sum_{i>j} \Im\left(U_{\alpha i}^{*} U_{\beta i} U_{\alpha j} U_{\beta j}^{*}\right) \sin ^{2}\left(\frac{\Delta m_{i j}^{2} L}{2 E}\right),
\end{aligned}
$$

where $\delta_{\alpha \beta}$ is the Kronecker symbol and $i$ and $j$ are indices over the mass eigenstates. 


\subsubsection{Three-Family Neutrino Mixing}

Although only three neutrino flavor eigenstates have been observed in nature, along with their corresponding charged leptons, the exposition so far assumes an arbitrary number of flavor and mass eigenstates. If indeed there exists a fourth neutrino mass eigenstate, a linear combination of the four states can be written:

$$
\left|\nu_{s}\right\rangle=\sum_{i=1}^{4} U_{s i}^{*}\left|\nu_{i}\right\rangle .
$$

However, this $\nu_{s}$ neutrino does not have a charged lepton partner, so it cannot couple to the $W$ boson, and, from measurements of the width of $Z$ decays into neutrinos [22], only three definite neutrino flavors are observed. Therefore, either $\nu_{s}$ is a very massive neutrino, suppressing the $Z$ decays, or it does not couple to the weak interaction and can be seen as a "sterile" neutrino. Identical arguments can be applied for scenarios of five or more neutrino mass eigenstates. In the rest of this Chapter we assume three flavor eigenstates $\nu_{e}, \nu_{\mu}, \nu_{\tau}$ and three mass eigenstates $\nu_{1}, \nu_{2}, \nu_{3}$. In this case, the neutrino mixing matrix $U_{P M N S}$ is

$$
U_{P M N S}=\left(\begin{array}{ccc}
U_{e 1} & U_{e 2} & U_{e 3} \\
U_{\mu 1} & U_{\mu 2} & U_{\mu 3} \\
U_{\tau 1} & U_{\tau 2} & U_{\tau 3}
\end{array}\right)
$$

Following the parametrization commonly used for the Cabbibo-Kobayashi-Maskawa (CKM) mixing matrix for quarks [25, 26], we can rewrite $U$ as

$$
U=\left(\begin{array}{ccc}
c_{12} c_{13} & s_{12} c_{13} & s_{13} e^{-i \delta} \\
-s_{12} c_{23}-c_{12} s_{23} s_{13} e^{i \delta} & c_{12} c_{23}-s_{12} s_{23} s_{13} e^{i \delta} & s_{23} c_{13} \\
s_{12} s_{23}-c_{12} c_{23} s_{13} e^{i \delta} & -c_{12} s_{23}-s_{12} c_{23} s_{13} e^{i \delta} & c_{23} c_{13}
\end{array}\right),
$$

where $c_{i j}=\cos \theta_{i j}$ and $s_{i j}=\sin \theta_{i j}$. The rows of the $U$ matrix represent the neutrino flavor eigenstates in terms of mass eigenstates, whereas the columns give the mass eigenstates in terms of flavor eigenstates. The angles $\theta_{12}, \theta_{13}$ and $\theta_{23}$ can be seen as rotation angles between the flavor and mass eigenstates. $\delta$ is a complex phase and 
its value being non-zero is a necessary condition for $C P$ violation to be observed in the leptonic sector. The neutrino mixing matrix can also be expressed as a product of matrices, thus

$$
U=\left(\begin{array}{ccc}
1 & & \\
& c_{23} & s_{23} \\
& -s_{23} & c_{23}
\end{array}\right)\left(\begin{array}{cccc}
c_{13} & & s_{13} e^{-i \delta} \\
& 1 & \\
& & \\
s_{13} e^{i \delta} & & c_{13}
\end{array}\right)\left(\begin{array}{cccc}
c_{12} & s_{12} & \\
-s_{12} & c_{12} & \\
& & & \\
& & & 1
\end{array}\right)
$$

In this representation, we can associate each matrix to the experimental searches for neutrino oscillations. The first matrix depends on $\theta_{23}$, an angle measured by atmospheric and long baseline neutrino experiments; the second matrix depends on $\theta_{13}$, which can possibly be measured with reactor experiments or with long baseline experiments, as argued in this thesis; the third matrix is related to $\theta_{12}$, which can be measured by solar neutrino experiments and long baseline reactor experiments, sensitive to low energy neutrinos. These points will be further elaborated in later Sections.

Using Eq. 1.10 and Eq. 1.13, we can now explicitly calculate oscillation probabilities. In this thesis, we are mostly concerned with $\nu_{\mu} \rightarrow \nu_{e}$ oscillations. The full expression for the relevant oscillation probability is given by (cf. Ref. [27]):

$$
\begin{aligned}
& P\left(\nu_{\mu} \rightarrow \nu_{e}\right)=4 c_{13}^{2} s_{13}^{2} s_{23}^{2} \sin ^{2}\left(\frac{\Delta m_{13}^{2} L}{4 E}\right) \\
& \quad+8 c_{13}^{2} s_{12} s_{13} s_{23}\left[c_{12} c_{23} \cos (\delta)-s_{12} s_{13} s_{23}\right] \cos \left(\frac{\Delta m_{23}^{2} L}{4 E}\right) \sin \left(\frac{\Delta m_{13}^{2} L}{4 E}\right) \sin \left(\frac{\Delta m_{12}^{2} L}{4 E}\right) \\
& \quad-8 c_{13}^{2} c_{12} c_{23} s_{12} s_{13} s_{23} \sin (\delta) \sin \left(\frac{\Delta m_{23}^{2} L}{4 E}\right) \sin \left(\frac{\Delta m_{13}^{2} L}{4 E}\right) \sin \left(\frac{\Delta m_{12}^{2} L}{4 E}\right) \\
& \quad+4 s_{12}^{2} c_{13}^{2}\left[c_{13}^{2} c_{23}^{2}+s_{12}^{2} s_{23}^{2} s_{13}^{2}-2 c_{12} c_{23} s_{12} s_{23} s_{13} \cos (\delta)\right] \sin ^{2}\left(\frac{\Delta m_{12}^{2} L}{4 E}\right) .
\end{aligned}
$$

The first line of this parametrization contains the term driven by $\theta_{13}$, whereas the second and third line show the $C P$ violating terms, resulting from the imaginary component of Eq. 1.10. The last line is driven by the solar oscillation parameters. This cumbersome expression can be greatly simplified using the observational knowledge already gathered by neutrino experiments, as illustrated in the next Section. 


\subsubsection{One Mass Scale Approximation}

The neutrino observation data gathered in recent years indicates that two of the neutrino mass eigenstates are nearly degenerate in comparison with the third one, as illustrated in Fig. 1.2. Therefore, we can make the approximation $\Delta m_{12}^{2} \ll \Delta m_{23}^{2} \approx$

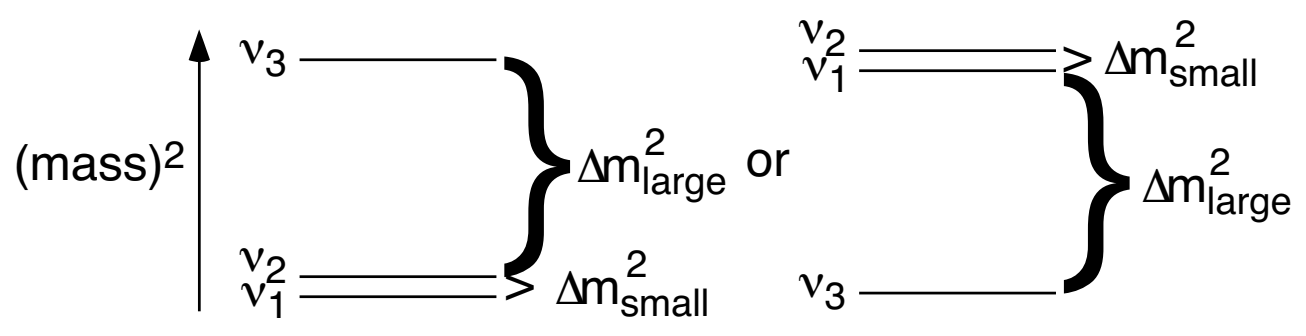

Figure 1.2: Mass spectral patterns for three neutrinos in which the $\nu_{2}-\nu_{1}$ splitting $\Delta m_{\text {small }}^{2}$ is much smaller than the splitting $\Delta m_{\text {large }}^{2}$ between $\nu_{3}$ and the $\nu_{2}-\nu_{1}$ pair. The left plot shows the spectrum for normal mass hierarchy $\left(\Delta m_{23}^{2}>0\right)$, whereas the right plot displays the spectrum for inverted hierarchy $\left(\Delta m_{23}^{2}<0\right)$.

$\Delta m_{13}^{2}=\Delta m^{2}$ [28]. If we factor in the so far omitted factors of $\hbar$ and $c$, and write $\Delta m^{2}$ in units of $\mathrm{eV}^{2}, L$ in $\mathrm{km}$ and $E$ in GeV, Eq. 1.15 becomes:

$$
P\left(\nu_{\mu} \rightarrow \nu_{e}\right)=\sin ^{2}\left(\theta_{23}\right) \sin ^{2}\left(2 \theta_{13}\right) \sin ^{2}\left(1.27 \frac{\Delta m^{2}}{E} L\right),
$$

where we used the identity $4 c_{13}^{2} s_{13}^{2}=\left[2 \sin \left(\theta_{13}\right) \cos \left(\theta_{13}\right)\right]^{2}=\sin ^{2}\left(2 \theta_{13}\right)$. Similarly, the dominant oscillation mode $P\left(\nu_{\mu} \rightarrow \nu_{\tau}\right)$ is given by

$$
P\left(\nu_{\mu} \rightarrow \nu_{\tau}\right)=\sin ^{2}\left(2 \theta_{23}\right) \cos ^{4}\left(\theta_{13}\right) \sin ^{2}\left(1.27 \frac{\Delta m^{2}}{E} L\right) .
$$

Finally, the survival probability $P\left(\nu_{\mu} \rightarrow \nu_{\mu}\right)$, important for the MINOS disappearance measurement, is simply:

$$
P\left(\nu_{\mu} \rightarrow \nu_{\mu}\right)=1-P\left(\nu_{\mu} \rightarrow \nu_{\tau}\right)-P\left(\nu_{\mu} \rightarrow \nu_{e}\right) .
$$

These expressions were used for all the necessary oscillation probability calculations carried out throughout the research work described in this thesis. 


\subsection{3 $C P$ Violation in the Leptonic Sector}

If we assume CPT conservation, we have [22]:

$$
P\left(\bar{\nu}_{\alpha} \rightarrow \bar{\nu}_{\beta}\right)=P\left(\nu_{\beta} \rightarrow \nu_{\alpha}\right)
$$

However, if we look carefully at Eq. 1.10 we may write

$$
P\left(\nu_{\beta} \rightarrow \nu_{\alpha} ; U\right)=P\left(\nu_{\alpha} \rightarrow \nu_{\beta} ; U^{*}\right)
$$

and so we find

$$
P\left(\bar{\nu}_{\alpha} \rightarrow \bar{\nu}_{\beta} ; U\right)=P\left(\nu_{\alpha} \rightarrow \nu_{\beta} ; U^{*}\right)
$$

Therefore, the equation giving the probability for oscillation of an anti-neutrino is the same as that of a neutrino, except the mixing matrix $U_{P M N S}$ is replaced by its complex conjugate. In this case, if $U_{P M N S}$ is not real, the last term in Eq. 1.10 will be different for neutrino and anti-neutrino oscillations. If $C P T$ is indeed conserved, then $C P$ violation (accompanied by a corresponding amount of $T$ violation) must occur. Let us take a more careful look into this matter.

In the general case of $n$ flavors, the mixing matrix $U_{P M N S}$ depends on $\frac{(n-1)(n-2)}{2}$ $C P$-violating complex phases [29]. In our case, $n=3$ and indeed, as we saw in Eq.1.13, we have only one $C P$-violating phase, $\delta$. If we keep the time dependency of Eq. 1.9 explicit we can write the probability of $\nu_{\alpha} \rightarrow \nu_{\beta}$ after some time $\Delta t=t-t_{0}$ as:

$$
P\left[\nu_{\alpha}\left(t_{0}\right) \rightarrow \nu_{\beta}(t)\right] \approx\left|\sum_{i} U_{\alpha i}^{*} e^{-i E\left(t-t_{0}\right)} U_{\beta i}\right|^{2} .
$$

Under $C P$ transformations, neutrinos are replaced by their anti-particles, which is equivalent to the complex conjugation of $U_{P M N S}$ :

$$
\begin{aligned}
C P: & \nu_{\alpha, \beta} \leftrightarrow \bar{\nu}_{\alpha, \beta} \\
& \Leftrightarrow U_{\alpha i}^{*} \rightarrow U_{\alpha i},(\delta \rightarrow-\delta) .
\end{aligned}
$$

The time reversal transformations $T$ interchange the initial and final evolution times $t$ and $t_{0}$ in Eq. 1.22 and can be seen as causing the evolution of the neutrino state to go "backwards in time". This is equivalent to the complex conjugation of the exponential 
factor in Eq. 1.22. However, the oscillation probability only depends on the modulus of the amplitude so the same result can be obtained by complex conjugation of the factors $U_{\alpha i}^{*}$ and $U_{\beta i}$, which in turn is equivalent to interchanging $\alpha$ and $\beta$. So, time reversal transformations can be interpreted as evolution forward in time, but between the interchanged initial and final neutrino flavors, thus:

$$
\begin{aligned}
T: t_{0} \leftrightarrow t & \Leftrightarrow \nu_{\alpha} \leftrightarrow \nu_{\beta} \\
& \Rightarrow U_{\alpha i}^{*} \rightarrow U_{\alpha i},(\delta \rightarrow-\delta) .
\end{aligned}
$$

When we combine $C P$ and $T$ transformations we then get:

$$
\begin{array}{cl}
C P T: & \nu_{\alpha, \beta} \leftrightarrow \bar{\nu}_{\alpha, \beta} \text { and } t_{0} \leftrightarrow t \quad\left(\nu_{\alpha} \leftrightarrow \nu_{\beta}\right) \\
& P\left(\nu_{\alpha} \rightarrow \nu_{\beta}\right) \rightarrow P\left(\bar{\nu}_{\beta} \rightarrow \bar{\nu}_{\alpha}\right)
\end{array}
$$

From $C P T$ invariance, it follows that $P\left(\nu_{\alpha} \rightarrow \nu_{\beta}\right)=P\left(\bar{\nu}_{\beta} \rightarrow \bar{\nu}_{\alpha}\right)$, a result essentially identical to the one in Eq. 1.19.

$C P$ and $T$ violations can be characterized by the probability differences:

$$
\begin{aligned}
\Delta P_{\alpha \beta}^{C P} & =P\left(\nu_{\alpha} \rightarrow \nu_{\beta}\right)-P\left(\bar{\nu}_{\alpha} \rightarrow \bar{\nu}_{\beta}\right) \\
\Delta P_{\alpha \beta}^{T} & =P\left(\nu_{\alpha} \rightarrow \nu_{\beta}\right)-P\left(\nu_{\beta} \rightarrow \nu_{\alpha}\right) .
\end{aligned}
$$

If $C P T$ invariance holds, the two probability differences are identical $\Delta P_{\alpha \beta}^{C P}=\Delta P_{\alpha \beta}^{T}$. Moreover, the survival probabilities will not display any $C P$ asymmetry:

$$
\Delta P_{\alpha \alpha}^{C P}=\Delta P_{\alpha \alpha}^{T}=0 \Rightarrow P\left(\nu_{\alpha} \rightarrow \nu_{\alpha}\right)=P\left(\bar{\nu}_{\alpha} \rightarrow \bar{\nu}_{\alpha}\right)
$$

This means flux disappearance measurements are completely insensitive to $C P$ violation. In the three neutrino case, where we only have one complex phase, $\delta$, we have a single probability difference

$$
\Delta P_{e \mu}^{C P}=\Delta P_{\mu \tau}^{C P}=\Delta P_{\tau e}^{C P} \equiv \Delta P
$$

where $\Delta P$ can be computed to be [29]:

$\Delta P=-4 s_{12} c_{12} s_{13} c_{13}^{2} s_{23} c_{23} \sin (\delta)\left[\sin \left(\frac{\Delta m_{12}^{2}}{2 E} L\right)+\sin \left(\frac{\Delta m_{23}^{2}}{2 E} L\right)-\sin \left(\frac{\Delta m_{13}^{2}}{2 E} L\right)\right]$.

This term vanishes when: 
i) One of the $\Delta m_{i j}^{2}$ is zero.

ii) One of the $\theta_{i j}$ is 0 or $90^{\circ}$.

iii) $\delta=0$ or $180^{\circ}$.

There is experimental evidence that $\theta_{13}$ is very small, which indicates that $C P$ violation in the leptonic sector will represent a very difficult measurement. The observation of $C P$ violation in the leptonic sector could shed new light on the problem of baryon asymmetry. The Sakharov conditions for the occurrence of the matterantimatter asymmetry [30] require the existence of $C P$ violation. However, the amount of $C P$ violation found in the hadronic sector is insufficient to account for the baryon asymmetry observed in the universe. It is argued that a mechanism of standard electroweak interactions mediated by sphalerons ${ }^{1}$ can interconvert baryons and leptons at temperatures above $1 \mathrm{TeV}$. If such mechanism exists, a net baryon asymmetry can be created by first creating a net lepton asymmetry at high temperatures through $C P$-violating processes [31].

The possibility of unveiling $C P$ violation is perhaps the main reason to study and measure $U_{e 3}=\sin \theta_{13} e^{-\delta}$. However, knowledge of $U_{e 3}$ can be interesting in many other ways. For instance, as we will illustrate later, $\theta_{13}$ is observed to be much smaller than the other two mixing angles for reasons that remain unexplained. $U_{e 3}$ is also the parameter that drives sub-dominant $\nu_{\mu} \rightarrow \nu_{e}$ neutrino oscillations and should provide the best opportunity of measuring oscillation enhancements due to matter effects, such as the coherent ones predicted by the Mikheev-Smirnov-Wolfenstein (MSW) effect $[32,33]$ or the ones resulting from parametric amplification of oscillations of corecrossing neutrinos inside the Earth [34]. The precise measurement of matter effects is the most powerful handle on determining the neutrino mass spectral ordering.

This thesis focuses on the potential of the MINOS experiment to measure $\theta_{13}$ or equivalently $\left|U_{e 3}\right|^{2}$ through observation of appearance of $\nu_{\mu} \rightarrow \nu_{e}$ oscillations at $\Delta m_{23}^{2}$. MINOS will not directly address most of the points mentioned above, but by

\footnotetext{
${ }^{1} \mathrm{~A}$ rare process predicted by the Standard Model occurring through weak interactions whereby three baryons can be converted to anti-leptons. The process violates baryon and lepton number conservation but conserves their difference (B-L). The process should be enhanced at high energies above $1 \mathrm{TeV}$.
} 
potentially performing the best measurement of or achieving the best limit on $\left|U_{e 3}\right|^{2}$, it will represent an encouraging prologue to the $U_{e 3}$ neutrino physics era.

\subsection{Experimental Evidence of Neutrino Oscillations}

Thanks to experimental results obtained during the last decade by increasingly precise neutrino measurements, the neutrino oscillation mechanism is now widely accepted as a viable explanation to deficits in neutrino flux observed by early experiments. In this Section, we summarize these results.

\subsubsection{Solar Neutrinos}

The nuclear fusion reactions within the Sun produce an intense flux of electron neutrinos. The combined effects of these reactions can be summarized in the process:

$$
4 p \rightarrow{ }^{4} \mathrm{He}+2 e^{+}+2 \nu_{e} .
$$

The average energy carried by the emerging neutrinos is $\left\langle E_{\nu}\right\rangle \sim 0.6 \mathrm{eV}$. A break down of the solar nuclear reactions that produce neutrinos is shown in the table in Fig. 1.3. The theoretical description of the Sun's structure and its evolution is commonly referred to as the Standard Solar Model (SSM). This model is found to be in excellent agreement with helio-seismological measurements. The SSM predictions for the several neutrino production channels are illustrated in Fig. 1.4.

\section{The Solar Neutrino Problem}

The first measurement of solar neutrinos was carried out by Davis et al. [35] in 1968, using a large container of tetrachloroethylene, essentially cleaning fluid. The container was located underground in the Homestake mine. The electron neutrinos are observed via the reaction:

$$
\nu_{e}+{ }^{37} \mathrm{Cl} \rightarrow e^{-}+{ }^{37} \mathrm{Ar}{ }^{*}
$$

The number of interactions is determined via a filtration and counting process carried out every few weeks. The solar neutrino flux measured by the Homestake experiment showed a deficit compared to the SSM expectation. These results were confirmed by 


\begin{tabular}{lcccc}
\hline \hline & & \multicolumn{4}{c}{$\mathrm{BP} 2000$} \\
\cline { 3 - 5 } \multicolumn{1}{c}{ Reaction } & Abbr. & Flux $\left(\mathrm{cm}^{-2} \mathrm{~s}^{-1}\right)$ & $\mathrm{Cl}\left(\mathrm{SNU}^{*}\right)$ & $\mathrm{Ga}\left(\mathrm{SNU}^{*}\right)$ \\
\hline$p p \rightarrow d e^{+} \nu$ & $p p$ & $5.95\left(1.00_{-0.01}^{+0.01}\right) \times 10^{10}$ & - & 69.7 \\
$p e^{-} p \rightarrow d \nu$ & $p e p$ & $1.40\left(1.00_{-0.015}^{+0.015}\right) \times 10^{8}$ & 0.22 & 2.8 \\
${ }^{3} \mathrm{He} p \rightarrow{ }^{4} \mathrm{He} e^{+} \nu$ & $h e p$ & $9.3 \times 10^{3}$ & 0.04 & 0.1 \\
${ }^{7} \mathrm{Be} e^{-} \rightarrow{ }^{7} \mathrm{Li} \nu+(\gamma){ }^{7} \mathrm{Be}$ & $4.77\left(1.00_{-0.10}^{+0.10}\right) \times 10^{9}$ & 1.15 & 34.2 \\
${ }^{8} \mathrm{~B} \rightarrow{ }^{8} \mathrm{Be}^{*} e^{+} \nu$ & ${ }^{8} \mathrm{~B}$ & $5.05\left(1.00_{-0.16}^{+0.20}\right) \times 10^{6}$ & 5.76 & 12.1 \\
${ }^{13} \mathrm{~N} \rightarrow{ }^{13} \mathrm{C} e^{+} \nu$ & ${ }^{13} \mathrm{~N}$ & $5.48\left(1.00_{-0.17}^{+0.21}\right) \times 10^{8}$ & 0.09 & 3.4 \\
${ }^{15} \mathrm{O} \rightarrow{ }^{15} \mathrm{~N} e^{+} \nu$ & ${ }^{15} \mathrm{O}$ & $4.80\left(1.00_{-0.19}^{+0.25}\right) \times 10^{8}$ & 0.33 & 5.5 \\
${ }^{17} \mathrm{~F} \rightarrow{ }^{17} \mathrm{O} e^{+} \nu$ & ${ }^{17} \mathrm{~F}$ & $5.63\left(1.00_{-0.25}^{+0.25}\right) \times 10^{6}$ & 0.0 & 0.1 \\
\hline \multicolumn{7}{c}{ Total } & & \multicolumn{3}{c}{$7.6_{-1.1}^{+1.3}$} & $128_{-7}^{+9}$ \\
\hline \hline
\end{tabular}

* $1 \mathrm{SNU}$ (Solar Neutrino Unit) $=10^{-36}$ captures per atom per second.

Figure 1.3: Neutrino producing reactions in the Sun and their corresponding fluxes. The predicted event rates for chlorine and gallium experiments is also shown. Plot from Ref. [22].

the two gallium experiments SAGE [36] and GALLEX [37] (GALLEX was renamed GNO for the most recent runs) and the water Čerenkov experiments Kamiokande [38] and Super-Kamiokande [39]. Given that all efforts to consistently accommodate these results within the SSM were unsuccessful, the solar neutrino anomaly was established. SAGE and GALLEX are conceptually similar to the Homestake experiment, but the use of gallium instead of chlorine allows for a lower detection energy threshold, conferring sensitivity to $p p$ neutrinos. The electron neutrinos react with gallium in the process:

$$
\nu_{e}+{ }^{71} G a \rightarrow e^{-}+{ }^{71} G e^{*} .
$$

The Homestake, SAGE and GALLEX experiments are collectively referred to as radiochemical experiments.

The Kamiokande detectors are located underground in the Kamioka mine and consist of large water tanks with the walls covered by photo multiplier tubes (PMTs). The principle of detection is based on collection of Cerenkov light emmitted as particles propagate in the water tank with speeds higher than the speed of light in the 


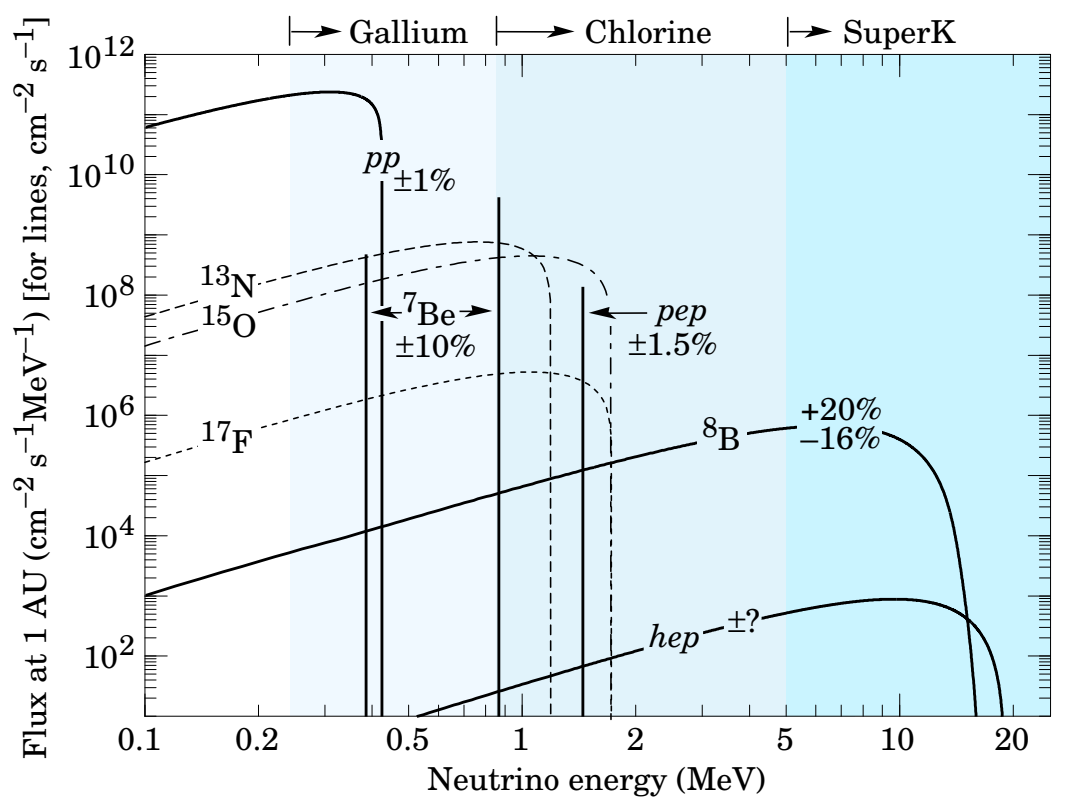

Figure 1.4: The solar neutrino energy spectrum predicted by the SSM. The energy ranges measurable by each of the main solar neutrino experiments are illustrated by the three colored energy bands. Plot from Ref. [22].

water. The Kamiokande detector has a mass of 3 kton and uses 980 PMTs. The Super-Kamiokande detector has a mass of 50 kton and employs 11000 PMTs. The Čerenkov light emission is triggered by recoil electrons from neutrino elastic scattering:

$$
\nu+e^{-} \rightarrow \nu+e^{-}
$$

The Kamiokande measurements should be dominated by electron neutrino interactions, as both $\mathrm{CC}$ and $\mathrm{NC}$ interactions contribute to the elastic scattering process. However, other neutrino flavors will also be measured via their NC interactions (but not distinguished from $\nu_{e}$-induced events).

\section{Evidence of solar neutrino oscillations}

The most recently commissioned solar neutrino experiment is the Sudbury Neutrino Observatory (SNO) [9]. The principle of detection is also based on Čerenkov light collection, but the medium used consists of heavy water $\mathrm{D}_{2} \mathrm{O}$. The detector, containing 1 kton of heavy water, is located $2000 \mathrm{~m}$ underground. The heavy water allows the 
distinction of charged current interactions, which occur for the electron neutrino only, and neutral current processes, which occur in the detector for all neutrino flavors. In regular water, the final states of a charged or neutral current interaction are the same, but in $D_{2} \mathrm{O}$ we have the reactions:

$$
\begin{gathered}
\nu_{e}+d \rightarrow e^{-}+p+p \\
\nu+d \rightarrow \nu+p+n
\end{gathered}
$$

SNO can also measure elastic scattering processes. Those measurements are used as a consistency check for the CC and NC results. SNO has the capability of measuring the total flux of all neutrino flavors combined via NC interactions, while simultaneously measuring the $\nu_{e}$ flux via the CC interactions. Given that SNO observes roughly $\phi_{\nu_{e}} / \phi_{t o t a l} \sim 1 / 3$ and we know that solar neutrinos are only of electron flavor, this result is an unambiguous proof that neutrino flavor change occurred during the $10^{11} \mathrm{~km}$ traveled by the solar neutrinos. A viewgraph summarizing the results obtained by the solar neutrino experiments compared to the SSM prediction is displayed in Fig. 1.5. Assuming the flavor change process undergone by the solar neutrinos ob-

Total Rates: Standard Model vs. Experiment Bahcall-Serenelli 2005 [BS05(OP)]

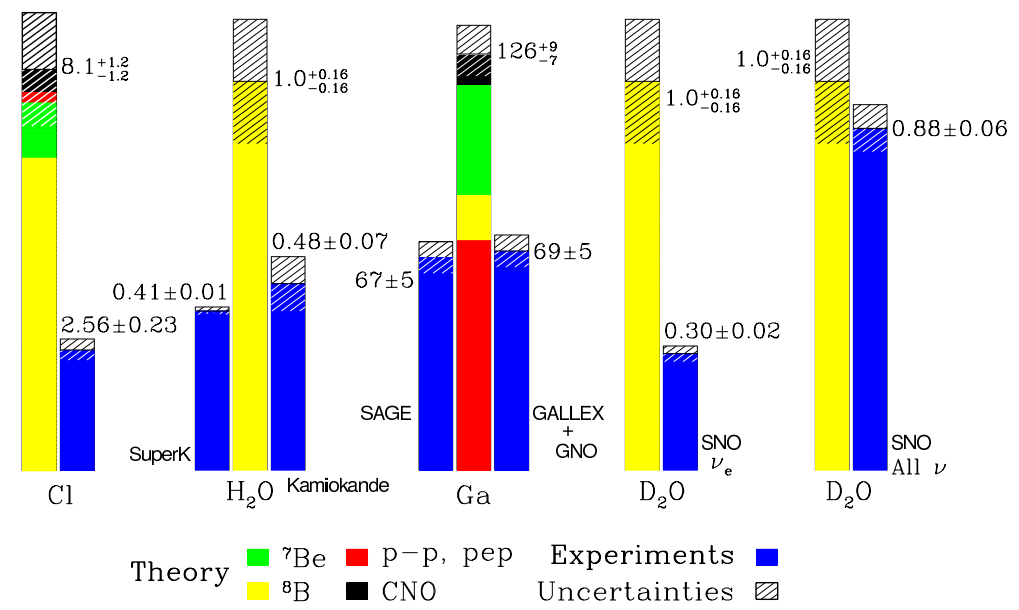

Figure 1.5: Summary of the solar neutrino flux measurement results as of 2005 compared to the SSM predictions. Plot obtained from Ref. [40]. 
served in SNO follows the neutrino oscillation phenomenology, a strong constraint is placed on the allowed range for the oscillation parameters $\theta_{12}$ and $\Delta m_{12}^{2}$, as shown in Fig. 1.6, which illustrates the combined fit of data from SNO plus all of the other solar neutrino experiments.

Albeit not purely a solar neutrino experiment, the very recent KamLAND experiment [41] is sensitive to the same range of oscillation parameters that may affect solar neutrino propagation. The KamLAND experiment is a 1 kton liquid scintillator detector located in Kamiokande's cavern. The solar neutrino program of KamLAND includes the measurement of the ${ }^{7} \mathrm{Be}$ and ${ }^{8} \mathrm{~B}$ solar neutrinos at a low energy threshold. However, the uniqueness of the experiment lies in its capability of carrying out a "ground" measurement of the solar neutrino oscillation parameters by performing long baseline (flux-weighted average distance of $180 \mathrm{~km}$ ) measurements of the flux of electron anti-neutrinos emitted by nuclear reactors. The reaction used is:

$$
\bar{\nu}_{e}+p \rightarrow e^{+}+n
$$

If CPT invariance holds and the LMA (Large Mixing Angle) solution preferred by SNO is valid, KamLAND should observe reactor $\bar{\nu}_{e}$ disappearance. In fact the first results showed a ratio $\frac{N_{o b s}-N_{B G}}{N_{N o O s c}}=0.611 \pm 0.085 \pm 0.041$, demonstrating clear evidence of the event deficit expected from neutrino oscillations. The resulting combined fit of all of the solar experiments and KamLAND [42] is displayed in Fig. 1.6.

The combined best fit point for the solar oscillation parameters was found to be:

$$
\begin{aligned}
\Delta m_{12}^{2} & =7.1_{-0.6}^{+1.2} \times 10^{-5} \mathrm{eV}^{2} \\
\theta_{12} & =32.5_{-2.3}^{+2.4} \text { degree }
\end{aligned}
$$

\subsubsection{Atmospheric Neutrinos}

The origins of the detection of the atmospheric neutrino anomaly, a deficit in the observed flux of atmospheric neutrinos when compared to the theoretically expected values, is traced back to experiments with the main goal of looking for proton decay. For these experiments, the atmospheric neutrino flux is an important source of background, so its precise measurement was a necessity but also a great contribution to neutrino physics. 

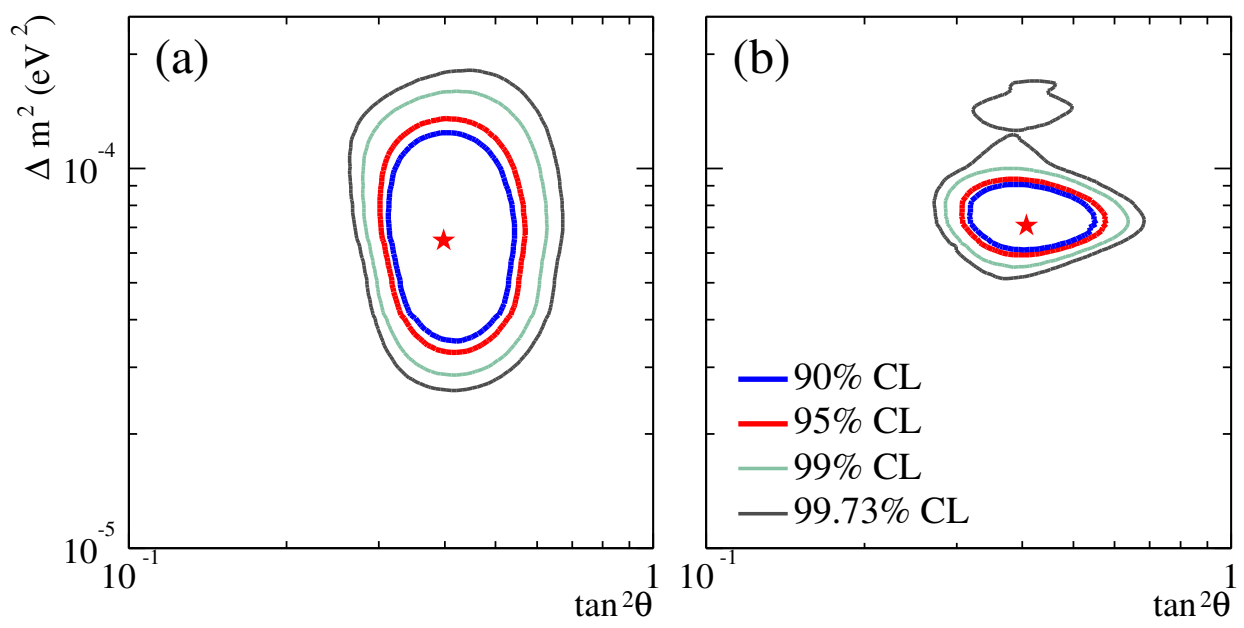

Figure 1.6: Solar neutrino oscillation allowed regions for $\theta_{12}$ and $\Delta m_{12}^{2}$. In (a), the global result obtained from the combination of the data of all solar neutrino experiments is shown, whereas in (b) the combined Solar+KamLAND result is displayed. In both cases the best fit point is identified by a star.

The atmospheric neutrinos result from the interaction of cosmic rays in the upper atmosphere (at an average of $\sim 20 \mathrm{~km}$ of altitude). These interactions produce kaons, pions and muons that give rise to neutrinos when they decay. For instance, the pion chain

$$
\begin{aligned}
p+N & \rightarrow \pi^{ \pm}+X \\
\pi^{ \pm} & \rightarrow \mu^{ \pm}+\nu_{\mu}\left(\bar{\nu}_{\mu}\right) \\
\mu^{ \pm} & \rightarrow e^{ \pm}+\nu_{e}\left(\bar{\nu}_{e}\right)+\nu_{\mu}\left(\bar{\nu}_{\mu}\right)
\end{aligned}
$$

is expected to produce neutrinos with a ratio $R=\frac{\nu_{\mu}+\bar{\nu}_{\mu}}{\nu_{e}+\bar{\nu}_{e}} \approx 2$. The IMB [43] and Kamiokande experiments were the first to observe a significant deviation from this value. Other experiments followed suit, as described in the review paper by Mann [44] and references therein. A summary plot of these early results is shown in Fig. 1.7.

The definite evidence for neutrino oscillation as the explanation for muon neutrino disappearance in atmospheric neutrinos was obtained by the Super-Kamiokande experiment (SuperK) [7], an experiment we described in the previous Section. This 


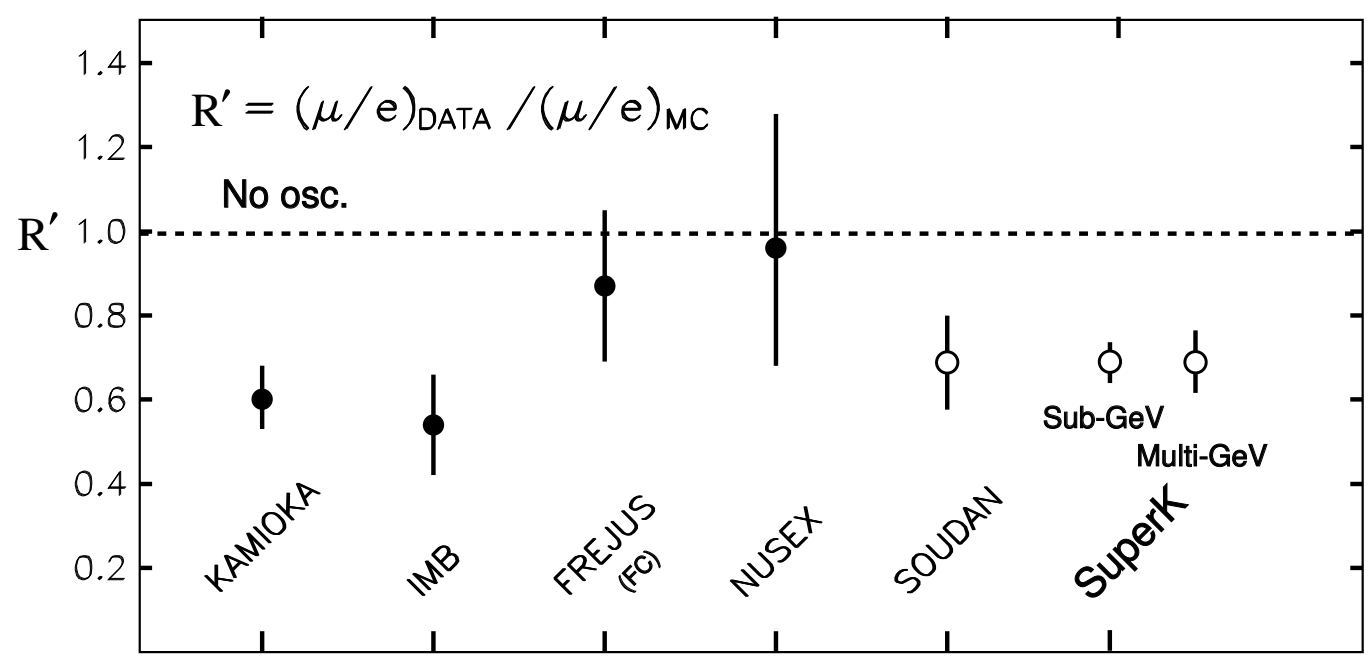

Figure 1.7: Atmospheric neutrino flux measurements from several experiments. $R^{\prime}$ is the ratio of ratios $R^{\prime}=R_{D A T A} / R_{M C}$, where $R$ corresponds to the definition given in the text. Plot from Ref. [44].

evidence is based on the angular distribution of neutrino events. Atmospheric neutrinos can have a very wide range of path lengths $L$, from $20 \mathrm{~km}$ up to $12700 \mathrm{~km}$, if they were produced on the opposite side of the Earth. SuperK sees a depletion in the latter neutrinos, called "upward-going", corresponding to incoming zenith angles below horizon, whereas the "downward-going", above horizon, observed sample was within the expected values. The electron neutrino flux is also well described by the expected atmospheric flux. These observations, summarized in Fig.1.8, indicated $\nu_{\mu} \rightarrow \nu_{\tau}$ neutrino oscillations with parameters:

$$
\begin{aligned}
1.6 \times 10^{-3} \mathrm{eV}^{2} \leqslant \Delta m_{23}^{2} & \leqslant 3.9 \times 10^{-3} \mathrm{eV}^{2} \\
\sin ^{2}\left(2 \theta_{23}\right) & >0.92 .
\end{aligned}
$$

The SuperK result has been independently confirmed by the Soudan 2 experiment [8], a 963 ton iron tracking calorimeter located underground in the Soudan mine. Evidence of this type has also been presented by MACRO [46], a large planar tracking calorimeter of coarse granularity. However, the MACRO result is limited to the measurement of upward-going muons resulting from $\nu_{\mu}$ interactions in the 

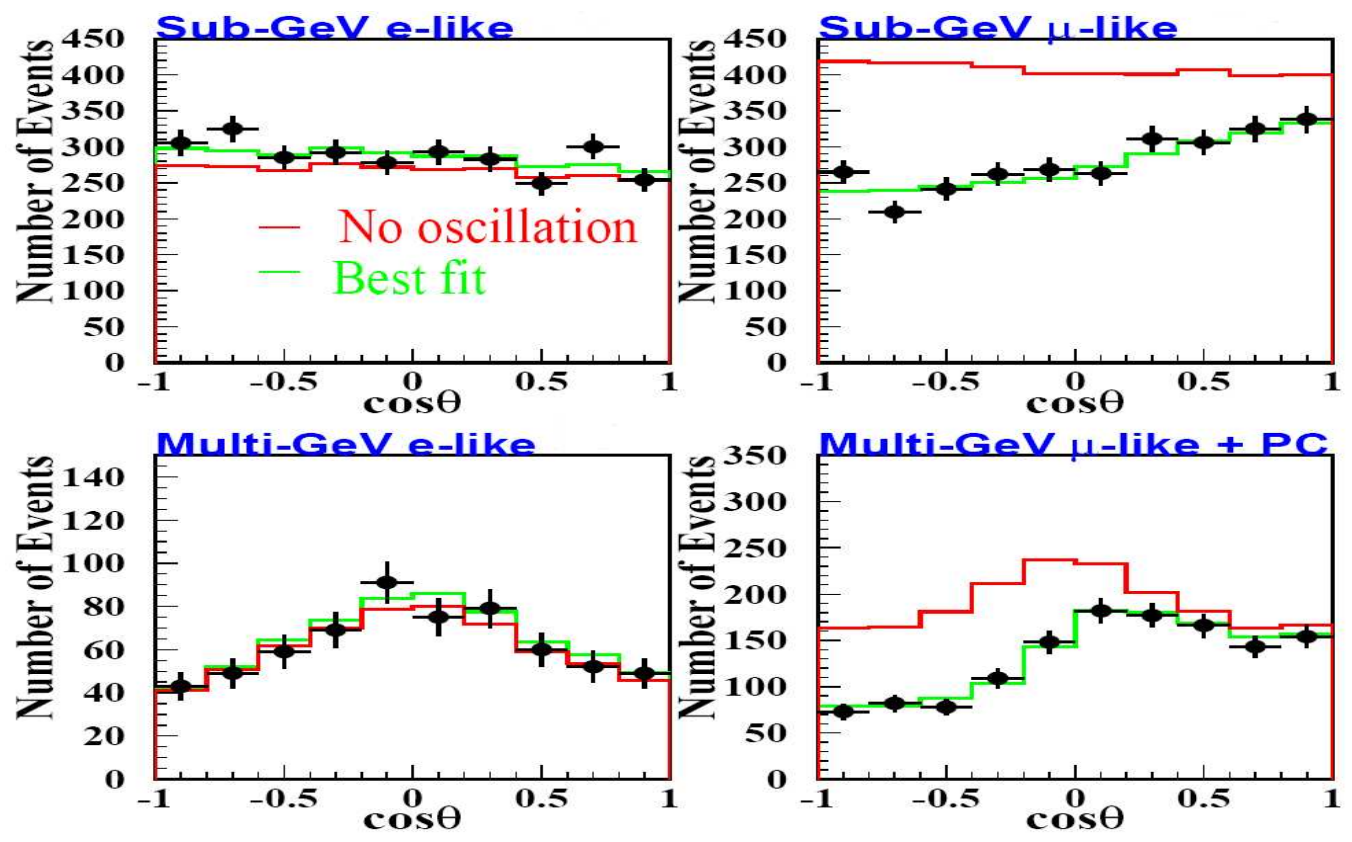

Figure 1.8: SuperK zenith angle distributions for the Sub-GeV and Multi-GeV samples of e-like and $\mu$-like events. For each plot, the black dots represent the data, the red line depicts the no-oscillation $\mathrm{MC}$ expected distribution, whereas the green line shows the MC distribution at the oscillation parameters best fit. Plot from Ref. [45].

rock surrounding the detector. The oscillation parameter allowed regions for SuperK, Soudan 2 and MACRO are displayed in Fig. 1.9.

The atmospheric oscillation parameter range can also be probed using long baseline accelerator experiments. One such experiment is K2K (KEK to Kamioka), which uses a neutrino beam, produced at the KEK accelerator and aimed at the SuperK detector, to search for flux disappearance by performing beam measurements at KEK and then at the SuperK location, after the neutrinos travel a distance of $250 \mathrm{~km}$. The results after a total beam intensity of $8.9 \times 10^{19}$ POT (protons-on-target) show a flux depletion of about 30\% [48].The allowed oscillation parameter region is consistent with the SuperK results.

The MINOS experiment follows the same concept as K2K, with a longer baseline of $735 \mathrm{~km}$ and an average beam energy of $\sim 3.5 \mathrm{GeV}$. However, a beam intensity considerably higher than that of $\mathrm{K} 2 \mathrm{~K}$ will allow for much better precision in the 


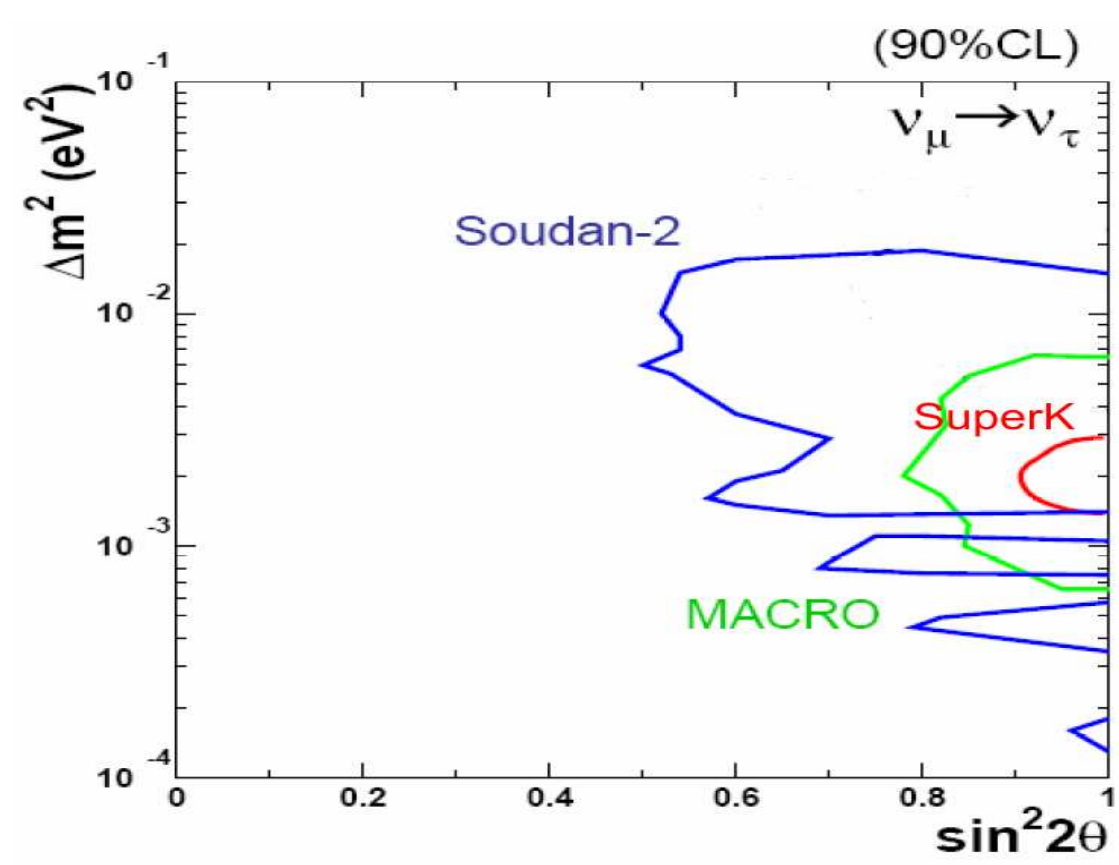

Figure 1.9: $90 \%$ contours for the atmospheric neutrino oscillation parameters $\theta_{23}$ and $\Delta m_{23}^{2}$. The SuperK allowed region is shown, along with the Soudan 2 and MACRO results for comparison. Plot from Ref. [47].

determination of the atmospheric oscillation parameters. MINOS will be discussed at length in Chapter 2.

\subsubsection{Looking for $\theta_{13}$}

The attempts to measure $\theta_{13}$ have been scarce and the existing results only provide limits on its magnitude. These limits are stringent enough to indicate that $\theta_{13}$ is very small and should therefore be particularly difficult to measure. The existing limits result from reactor experiments (see Ref. [49]). The strongest limit was set by the CHOOZ experiment [50].

The CHOOZ detector was built at the distances of $1115 \mathrm{~m}$ and $998 \mathrm{~m}$ from the two reactors of the Chooz nuclear power plant in France. The apparatus consisted of a central volume of scintillator with a mass of 5 tons, where the reactor $\bar{\nu}_{e}$ are detected. CHOOZ final results extracted from runs during 1997 are consistent with no $\bar{\nu}_{e}$ disappearance, which places an upper limit on $\theta_{13}$ that is stringent enough to 
rule out $\nu_{\mu} \rightarrow \nu_{e}$ oscillations as a significant contributor to the observed atmospheric neutrino deficit (confirmed to result from $\nu_{\mu} \rightarrow \nu_{\tau}$ by SuperK).

Another reactor experiment similar to $\mathrm{CHOOZ}$ was constructed near the Palo Verde nuclear reactor, in Arizona, USA. The Palo Verde results are completely consistent with $\mathrm{CHOOZ}$, albeit placing a weaker constraint on $\theta_{13}$. Finally, an oscillation analysis of SuperK data based on the one-mass-scale approximation previously described has been carried out [51]. A summary of the excluded oscillation parameter region by these results is presented in Fig. 1.10

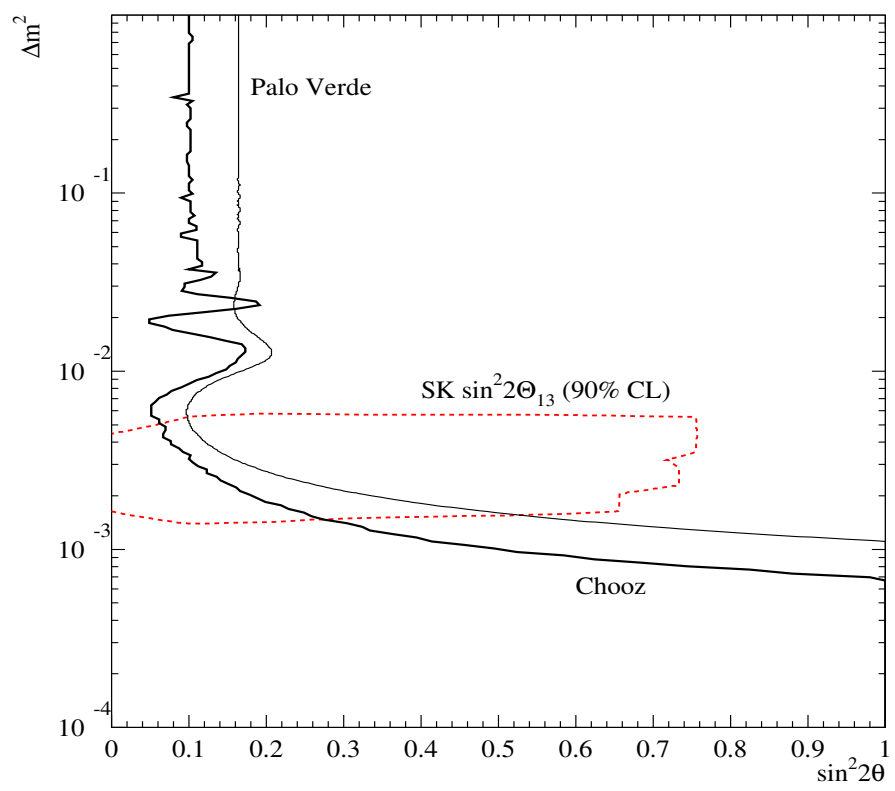

Figure 1.10: $90 \%$ excluded region of $\theta_{13}$ and $\Delta m_{23}^{2}$ for the reactor neutrino experiments (solid lines). The excluded regions are to the right of and above the $90 \%$ contours. The $90 \%$ allowed region obtained from the SuperK analysis is also shown corresponding to the region enclosed by the red dotted contour. Plot obtained from Ref. [49].

In this thesis, we will be mostly concerned with exploring the potential of MINOS in improving the $\mathrm{CHOOZ}$ limit on $\theta_{13}$. 


\subsubsection{Summary}

To complete the panorama of the evidence for neutrino oscillations referred to so far, we should briefly mention the LSND experiment [52], which looked for $\bar{\nu}_{e}$ appearance from a beam of $\bar{\nu}_{\mu}$ on a short baseline. LSND claimed to have observed a signal, which implies a $\Delta m^{2}$ value in the range of $0.2-10 \mathrm{eV}^{2}$, much larger than any value measured by other experiments to date. This result represents a third mass splitting which would require a fourth neutrino. As we have discussed in previous Sections, such a neutrino would be sterile. The LSND result is very controversial and remains unconfirmed. The Mini-Boone experiment [53] at Fermilab, Illinois, USA, will be able to confirm or rule out the LSND result very soon (2006).

The complete picture of experimental evidence of neutrino oscillations is shown in Fig. 1.11

Global fits performed by Fogli [54] to all the results available in 2004 indicate the following:

$$
\begin{aligned}
\Delta m_{\text {Small }}^{2} & =8.0_{-0.7}^{+0.8} \times 10^{-5} \mathrm{eV}^{2} \\
\Delta m_{\text {Large }}^{2} & =2.4_{-0.6}^{+0.5} \times 10^{-3} \mathrm{eV}^{2} \\
\sin ^{2} \theta_{12} & =0.29_{-0.04}^{+0.05} \\
\sin ^{2} \theta_{23} & =0.45_{-0.11}^{+0.18} \\
\sin ^{2} \theta_{13} & \leqslant 0.035 .
\end{aligned}
$$

These results can be summarized by showing their effects on constraining the neutrino mixing matrix, effects that can be sketched as in Fig. 1.12.

We have painted the present day picture of neutrino oscillation physics. In the next and final Section of this Chapter, we look at the next steps to be taken.

\subsection{Future Neutrino Research}

As discussed in Section 1.2.3, $U_{e 3}$ studies are the current driving goal of experimental neutrino physics. Measuring $U_{e 3}$ is the key to understanding $C P$ violation in the 


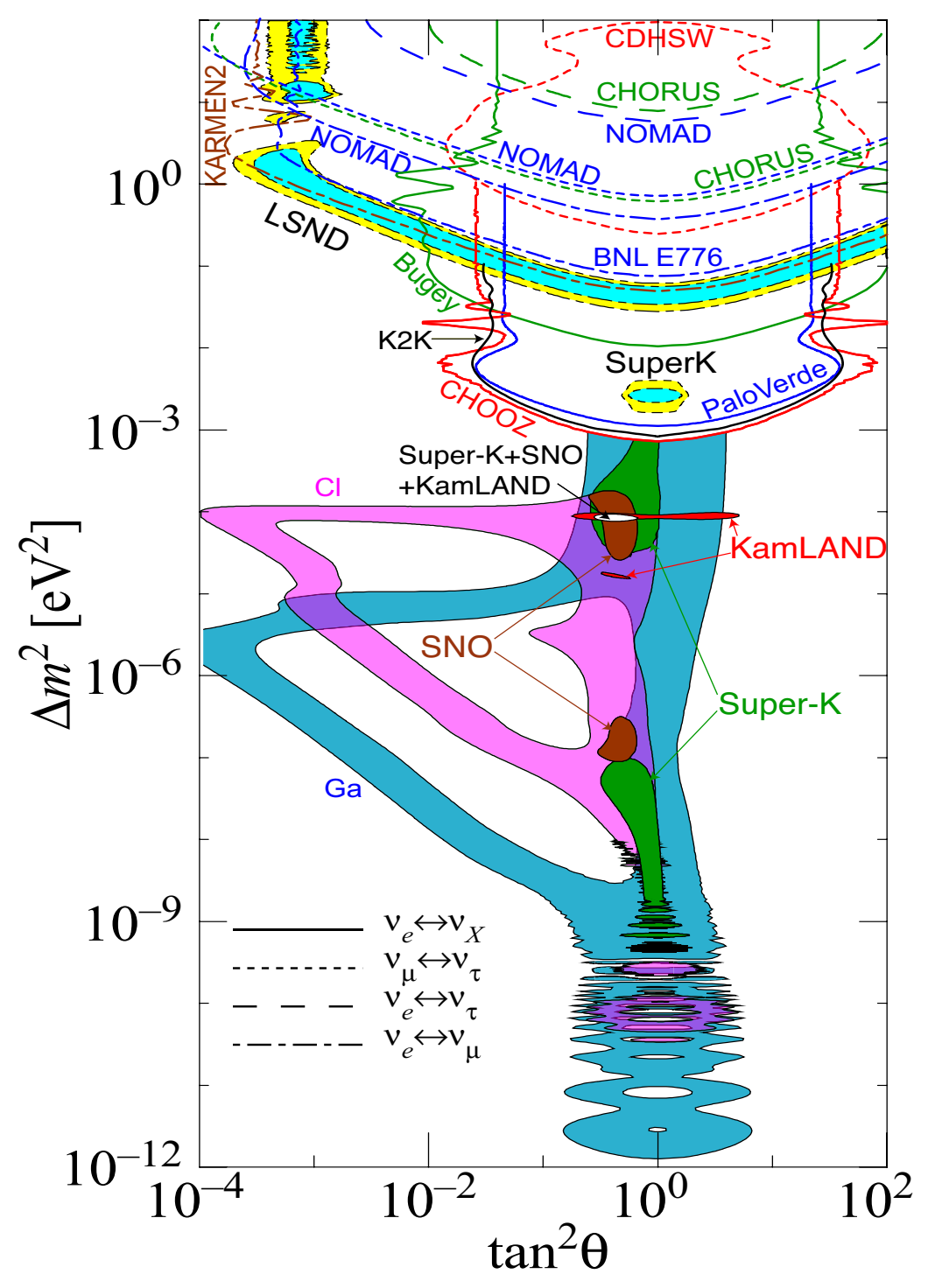

http://hitoshi.berkeley.edu/neutrino

Figure 1.11: The regions of squared mass splitting and mixing angle favored or excluded by various experiments. Figure compiled by H. Murayama (See Ref. [22]).

leptonic sector and to determining the neutrino mass hierarchy. The next generation of reactor experiments will start with Double-CHOOZ [56], using the same reactors and a similar concept to that of $\mathrm{CHOOZ}$, but making use of two detectors at different distances. Double-CHOOZ expects to start operating in 2008 and to reach a sensitivity of $\sin ^{2} 2 \theta_{13}<0.03$ at $90 \%$ confidence level for $\Delta m_{\text {atm }}^{2}=2.0 \times 10^{-3} \mathrm{eV}^{2}$ after three 


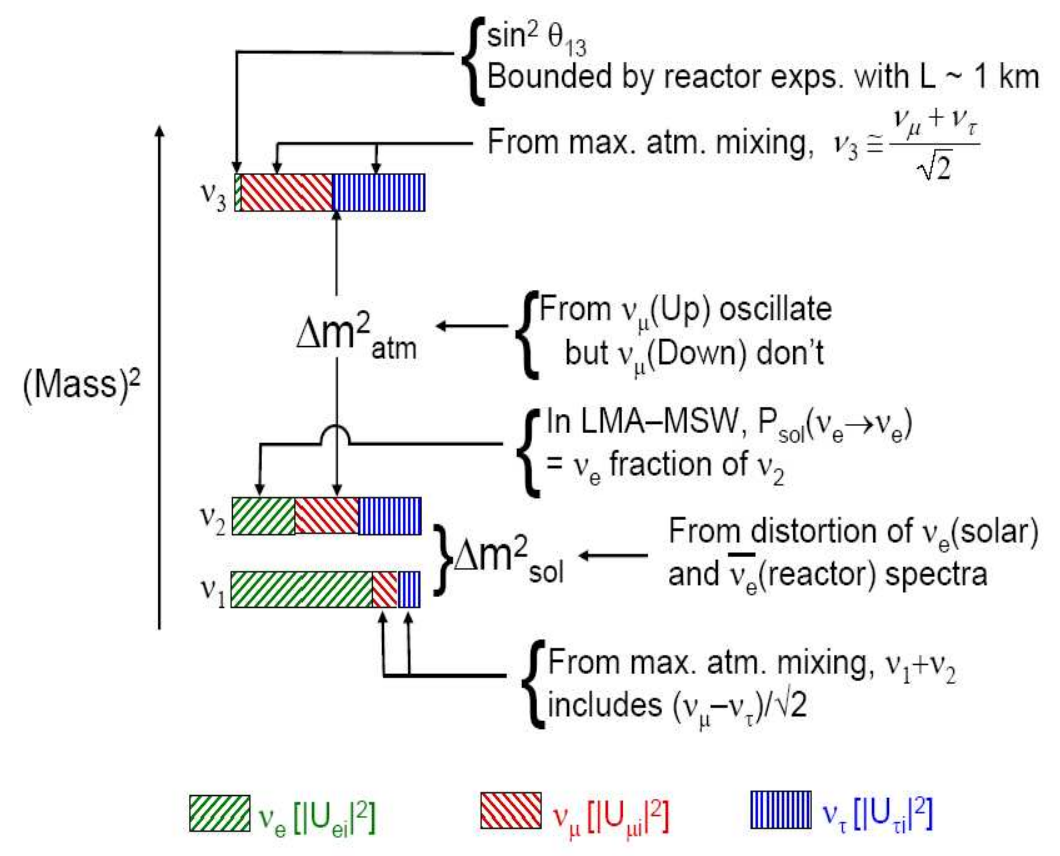

Figure 1.12: A three neutrino mass spectrum summarizing the effects of experimental results on our knowledge of the neutrino mixing matrix. The ordering of the mass eigenstates follows the normal hierarchy in increasing value of $m^{2}\left(\nu_{1}, \nu_{2}, \nu_{3}\right)$, but an analogous plot is trivially obtained for an inverted hierarchy $\left(\nu_{3}, \nu_{1}, \nu_{2}\right)$. Plot from Ref. [55].

years of running. This would represent an improvement of roughly 5 times on the CHOOZ bound. However, being a disappearance experiment, Double-CHOOZ will be blind to $C P$-violating effects. The first experiments able to probe this physics, NOvA [57] and T2K [58], are already in a state of active R\&D.

The NOvA (NuMI Off-Axis $\nu_{e}$ Appearance) experiment will share the NuMI beam with the MINOS experiment and use a 30 kton totally active liquid scintillator far detector placed $\sim 800 \mathrm{~km}$ from the beam origin on an off-axis position. The off-axis positioning provides a narrow beam energy spectrum, while the low density of the far detector enhances showering event measurements, fundamental in a $\nu_{e}$ appearance experiment. These specifications should allow NOvA to improve on CHOOZ by an order of magnitude after three years of running. If $\nu_{e}$ appearance is indeed observed, NOvA could then switch to running with an anti-neutrino beam, thus measuring 
matter effects and possibly providing the first hints on the nature of the neutrino mass hierarchy. The ability of NOvA to measure $\delta$, the $C P$-violating phase, will depend on the ability to isolate the matter effects component and resolve the mass hierarchy. Finally, thanks to very large statistics and good energy resolution, NOvA should be able to measure $\theta_{23}$ with a precision an order of magnitude better than that for the existing SuperK measurement. NOvA's construction is planned to start in 2007 and the first data to be taken in 2009 .

The T2K (Tokai to Kamioka) experiment will use a 0.77 MW neutrino beam produced at the JHF lab pointed at the Super-Kamiokande detector, $295 \mathrm{~km}$ away. The first phase of T2K will look for $\nu_{\mu} \rightarrow \nu_{e}$ oscillations, potentially placing a constraint on $\theta_{13}$ comparable to that of NOvA. In a second phase, the neutrino beam is upgraded to $4 \mathrm{MW}$ (which would become the first so-called super $\nu$ beam) and the far detector would be the proposed Hyper-K, a 1 Mton water Čerenkov detector. The first stage of the project is scheduled to start in 2008-09. If $\nu_{\mu} \rightarrow \nu_{e}$ is observed in the first stage, T2K could be able to measure $C P$ asymmetry by running also with $\bar{\nu}_{\mu}$. Because of the shorter baseline, matter effects are not as important as in the case of NOvA, and T2K's measurement might be better. However for the same reasons, T2K's sensitivity to the mass hierarchy is reduced compare to NOvA's.

As can be seen, the next decade promises many interesting results and possible discoveries, perhaps even surprises, something that in a period of 75 years has become all but the rule in neutrino physics. 


\section{Chapter 2}

\section{The MINOS Experiment}

The Main Injector Neutrino Oscillation Search experiment utilizes the NuMI (Neutrinos from the Main Injector) beam, measuring the neutrino signal $800 \mathrm{~m}$ downstream from production and $734 \mathrm{~km}$ further with similar Near and Far planar steel and scintillator detectors located at Fermilab, Batavia, Illinois, and at the Soudan Underground Laboratory, Soudan, Minnesota.

MINOS

Long-baseline experiment at Fermilab

Near Detector at NuMI

EERMILAB IIlnots

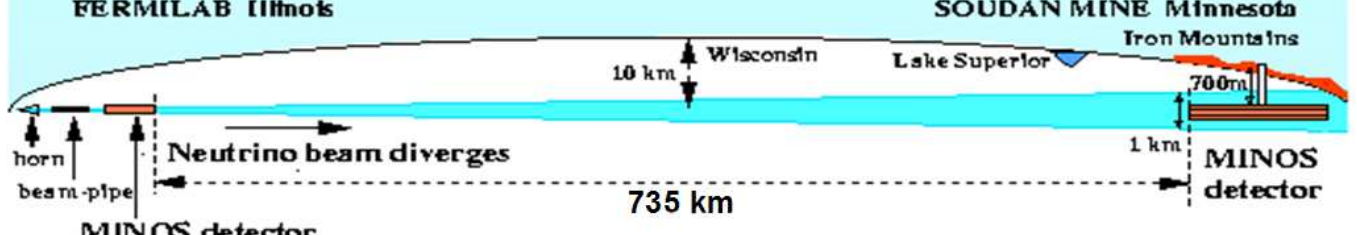

Figure 2.1: MINOS experiment overview. Relative locations of the two MINOS detectors and the neutrino beam origin are shown (not to scale).

In March 1991, the P822 collaboration, that would later become the MINOS collaboration, proposed a long baseline neutrino oscillation experiment from Fermilab to Soudan [59]. The Soudan 2 detector [60] was the originally intended neutrino beam target. By June 1993, the P822 proposal was updated to include the construction 
of another detector in a new cavern adjacent to the existing Soudan 2 cavern. The NuMI/MINOS collaboration was officially born in February 1995 with the proposal P875, titled "A Long-Baseline Neutrino Oscillation Experiment at Fermilab" [61]. The experiment was funded by the Department of Energy in November 1997 and construction started in June 1999. The MINOS Far Detector was completed in July 2003 and the Near Detector was fully assembled and commissioned by September 2004. The first neutrinos from NuMI were observed in the Near Detector in January 2005. During the construction phase, a scaled down version of the MINOS detectors, CalDet, was built at CERN, Switzerland and used with two test beams to calibrate the various detector systems and estimate detector responses. At the time of writing of this thesis, MINOS is regularly taking beam neutrino data and a total intensity of $1 \times 10^{20}$ protons-on-target has been collected.

This chapter describes the major components of the MINOS experiment and outlines its physics reach. More detailed descriptions can be found in [62].

\subsection{The NuMI Beam}

The NuMI neutrino beam results from the decays of secondary pions and kaons produced by $120 \mathrm{GeV}$ protons extracted from the Main Injector once per each cycle of $1.9 \mathrm{~s}$, for a spill duration of $8.7 \mu \mathrm{s}$. The Main Injector delivers an intensity of $2.5 \times 10^{13}$ protons per spill that are focused on a graphite target. The positively charged hadrons produced by proton collisions in the target are focused into a beam by two parabolic magnetic horns. These horns are energized with a $200 \mathrm{kA}$ current, The focused hadrons then decay within a $675 \mathrm{~m}$ long, $2 \mathrm{~m}$ diameter pipe evacuated to less than 1 torr. Most decays produce positive muons and muon neutrinos, but decays into positrons and electron neutrinos occur in $1 \%$ of the cases. A section of absorber (concrete) and rock downstream of the decay pipe stops hadrons that have not decayed. The surviving muons range out in the $240 \mathrm{~m}$ of rock interposed between the hadron absorber and the Near Detector, located $~ 800$ m downstream of the target. A detailed schematic of the NuMI beamline is shown in Fig. 2.2.

The NuMI beam is unique in that it can be tuned to specific energy spectra. Both 


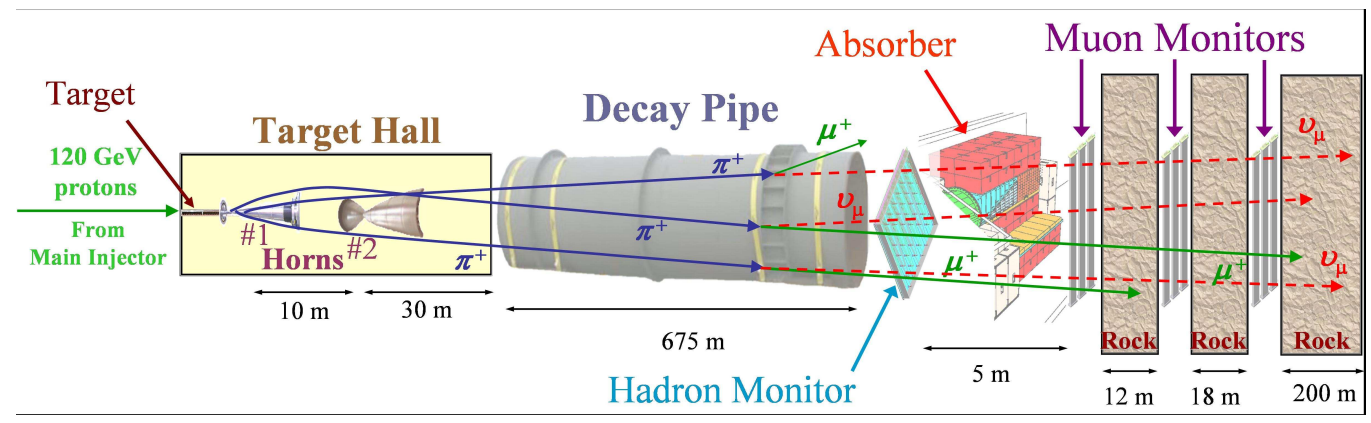

Figure 2.2: The layout of the NuMI beamline. Protons extracted from the Main Injector incident on a graphite target produce hadrons, focused by two parabolic horns, that then decay into muons and neutrinos. Any surviving hadrons and muons range out in a section of absorber and rock, as the neutrino beam continues towards the Near Detector (schematic by B. Zwaska).

the target and the second magnetic horn can be moved and, by varying their relative positions to each other and to the first horn, different beam configurations can be obtained. Configurations corresponding to the "Low", "Medium" and "High" energy spectra are depicted in Fig. 2.3. Additional target-horns relative positionings have been used for MINOS beam data taking, including placement of the target $1 \mathrm{~m}$ and $2.5 \mathrm{~m}$ behind the first horn, while keeping the second horn in the Low energy position, referred to as pseudo-Medium and pseudo-High energy configurations, respectively. During its first years of running, MINOS will predominantly use the Low energy configuration of the NuMI beam, which yields an expected beam spectrum at the Far Detector peaked around $3 \mathrm{GeV}$, thus optimizing the experiment's sensitivity to regions in oscillation parameter space constrained by SuperKamiokande and K2K (see Chapter 1). Detailed information concerning the NuMI beam is found in [63].

\subsection{The MINOS Detectors}

The MINOS detectors share a similar design in order to minimize systematic errors. This section describes the common design and technology aspects of the Near and Far detectors, followed by an overview of their particular characteristics and performance. 

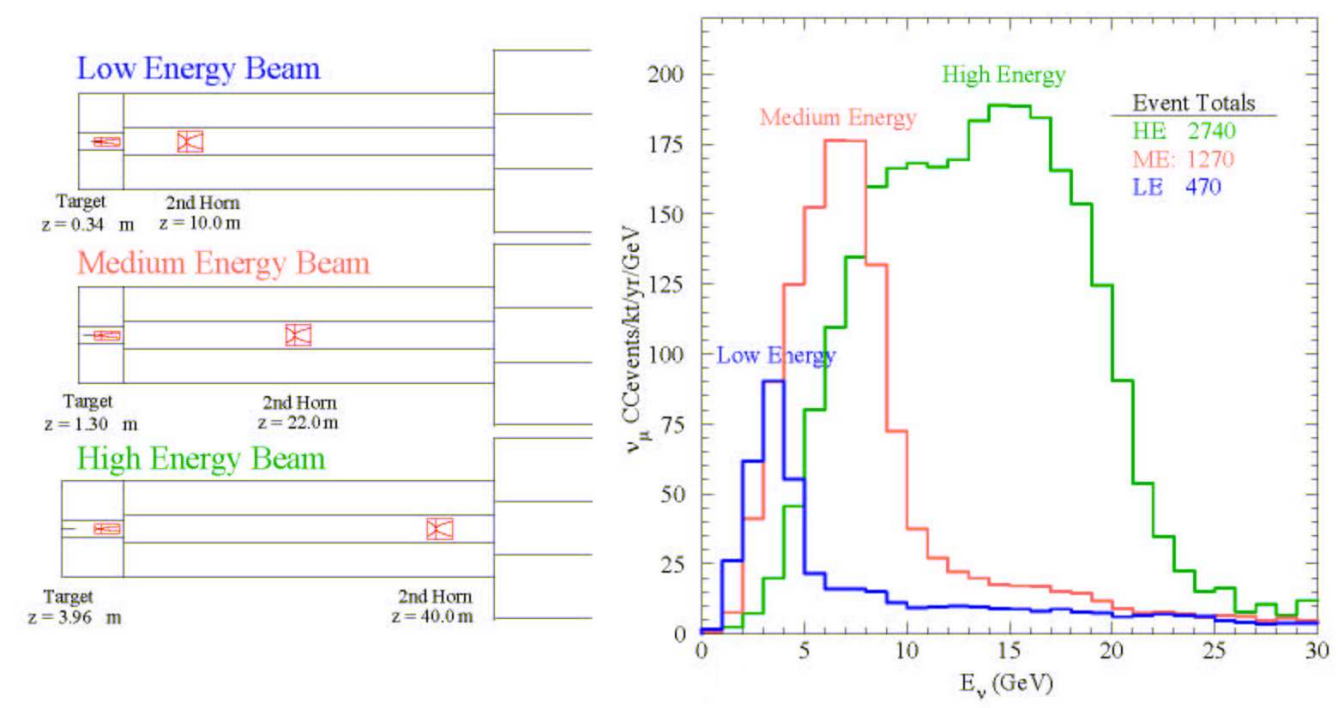

Figure 2.3: The NuMI beam spectra. Variation of the relative positions of the target and the horns, depicted in the left hand side, allows for tuning of the expected Far Detector beam $\nu_{\mu}$ energy spectrum, as shown on the right hand side.

\subsubsection{Detector Technology}

The MINOS detectors are sampling tracking calorimeters arranged as juxtaposed vertical planes, each one composed of an active layer of plastic scintillator backed by an absorber layer of steel. The scintillator consists of polystyrene infused with $1 \%$ PPO and 0.030\% POPOP fluors, shaped as $4.1 \mathrm{~cm}$ wide, $1 \mathrm{~cm}$ thick strips, co-extruded with a $\mathrm{TiO}_{2}$ reflective sheath. It is organized in six different types of modules, varying in length and containing a variable number of strips wrapped in a thin $0.5 \mathrm{~cm}$ thick aluminum skin. The absorber layer is made of $2.54 \mathrm{~cm}$ thick steel sheets. The steelscintillator planes are hung vertically on a mounting structure with an average interplanar distance or pitch of $5.95 \mathrm{~cm}$ [64], leaving an air gap between planes of $\sim 2.4 \mathrm{~cm}$. The orientation of the scintillator strips, making an angle of $45^{\circ}$ with the horizontal, is alternately rotated by $\pm 90^{\circ}$ with each successive plane, with the coordinate along the strip direction being called $\mathrm{U}$ or $\mathrm{V}$. This design, illustrated in Fig. 2.4, allows for 3D spatial positioning of particles traversing the detector. Neutrino interactions in the detector occur prevailingly in an absorber layer, producing secondary particles that escape the steel and traverse the scintillator strips, ionizing the base plastic. The 


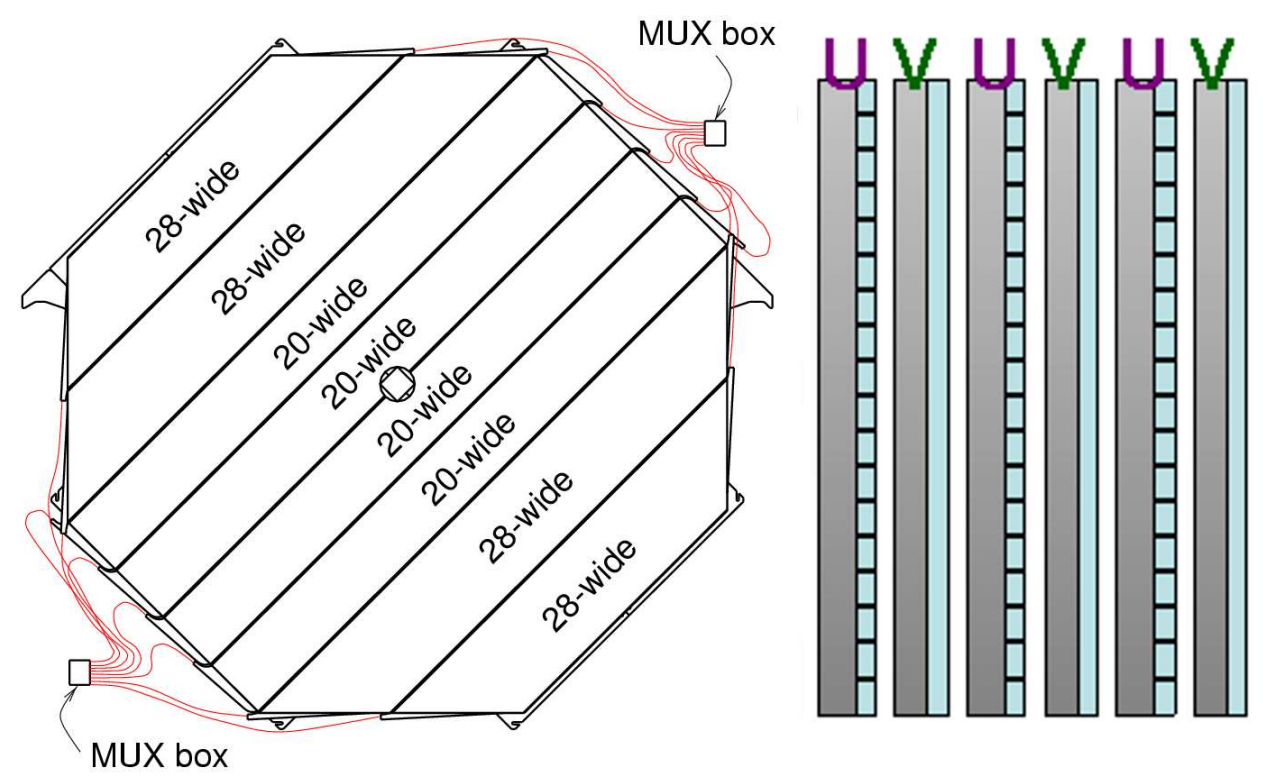

Figure 2.4: Diagram of a Far Detector MINOS scintillator plane. On the left-hand side the break-up of a scintillator plane in scintillator modules is shown. On the right hand-side, the longitudinal configuration of the steel-scintillator planes is depicted. Note the alternate strip orientation for $\mathrm{U}$ and $\mathrm{V}$ planes.

short wavelength radiation emitted by the excited molecules of the plastic excites the primary fluor, which produces ultra-violet radiation that excites the secondary fluor molecules. The secondary fluor emits longer wavelength blue light, which is collected by a Kuraray Y11 wavelength shifting fiber, with a diameter of $1.2 \mathrm{~mm}$, glued into a groove running the full length of the strip along its center. The fiber shifts the absorbed photons wavelength from blue to green and carries them to a manifold at the edge of the detector. From there, the light is carried by clear fiber cables to multi-anode photomultiplier tubes (PMTs), where it is converted to electric charge measured by front end electronics (FEE). The amount of charge measured is approximately proportional to the energy deposited in the detector by the neutrino interaction daughter particles. An image of the scintillator strips and of the PMTs is shown in Fig. 2.5.

Both of the MINOS detectors are magnetized with a field of $\sim 1.3 \mathrm{~T}$, on average, within the fiducial volume. The approximately toroidal field is produced by current 

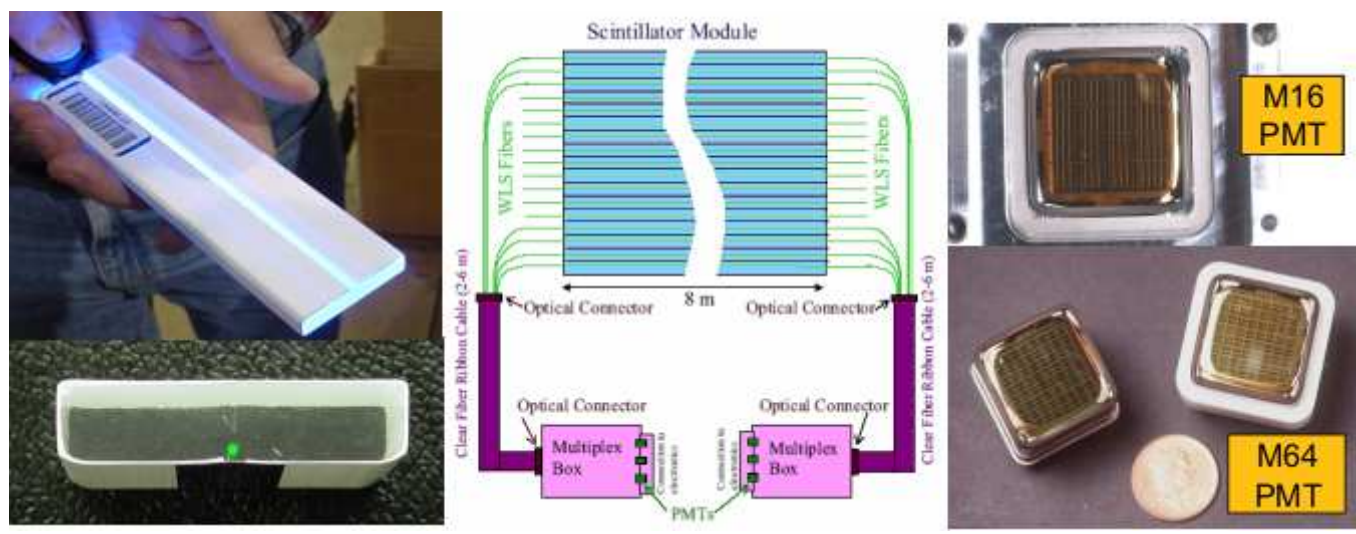

Figure 2.5: These images show sections of MINOS scintillator strips, with the WLS fiber apparent as the green dot in the bottom left image, a diagram of the scintillator light readout and the type of PMTs that read out the MINOS detectors, M16 and M64 for Far and Near, respectively.

carrying coils running through the center of the Far Detector, or offset from the center in the Near Detector case.

\subsubsection{The Far Detector}

The MINOS Far Detector is situated at a depth of $780 \mathrm{~m}$ below the surface $(2100 \mathrm{~m}$ water equivalent) in the Soudan Underground Laboratory, located on the $27^{\text {th }}$ level of the Soudan Underground Mine State Park. Placed at a distance of $735 \mathrm{~km}$ from beam production, the primary purpose of the Far Detector is to measure the oscillated neutrino beam spectum.

The total mass of the detector is $5.4 \mathrm{kt}$. The calorimeter is shaped as an $8 \mathrm{~m}$ tall, $30 \mathrm{~m}$ long octagonal prism with faces in the vertical position, and consists of 486 steel and scintillator planes organized in two supermodules. Supermodule 1 contains 250 planes, whereas supermodule 2 encompasses 236 planes. Each one of the supermodules is fitted with a $15 \mathrm{kA}$-turn coil running along its axis and through the center of each plane. An air gap of $1.5 \mathrm{~m}$ between planes 249 and 250 separates the supermodules and allows enough space for the return yokes of both coils. Fig. 2.6 shows a photograph of the completed MINOS Far Detector. The scintillator section of each plane contains 192 strips arranged in 8 scintillator modules, as shown in Fig. 2.4. The 


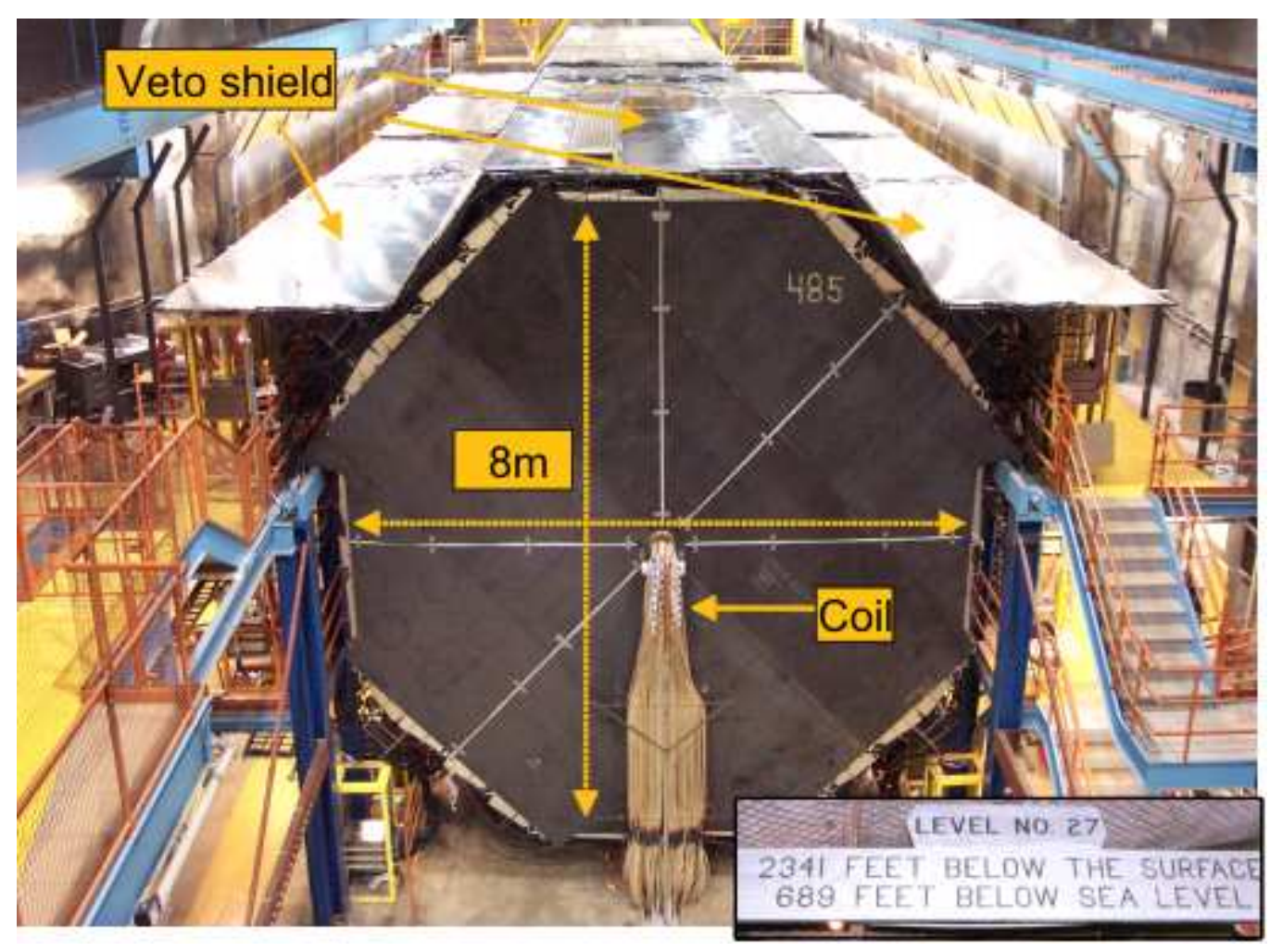

Figure 2.6: The completed MINOS Far Detector. On the sides of the detector, the yellow racks on the top and lower levels contain the MUX boxes that harbor the PMTs. The racks on the mezzanine level contain the readout electronics, high voltage mainframes, timing PCs and magnetic field monitoring computers.

first plane of each supermodule, plane 0 and plane 250 respectively, does not possess a scintillator portion and is therefore not instrumented. The scintillator strips are read out on both sides of the detector by Hamamatsu M16 photomultiplier tubes, with 16 output anodes, or pixels, and a common dynode signal. The signals are multiplexed so that 8 fibers are read out by a single PMT pixel. Demultiplexing is made possible by the usage of distinct multiplexing patterns for the readouts at each side of the detector. The PMT pixels are read out using modified Viking VA chips, produced by IDE AS of Norway. The chip contains signal shaping amplifiers and signal sampling and holding circuitry for each pixel. Three of these chips are housed in each VA Front-end Board (VFB), reflecting the fact that each box (referred to as MUX box) in the readout crates located in the upper and lower floors contains three PMTs. The 
analog signals are sent from a VFB to a VARC Mezzanine Module (VMM), where they are digitized. Six VMMs are encased on a VA Readout Control board (VARC), responsible for timestamps and control of the VA devices. Readout of the front-end electronics (FEE) is triggered by the PMT dynode signals, when one of the signals from the three PMTs connected to a VFB is higher than some threshold, generally $1 / 3$ of a photoelectron (PE). Following the dynode trigger activation, the signals from all the 16 pixels in the PMT are sent to the VARC and digitized. The Far Detector FEE provides a charge resolution of $2 \mathrm{fC} /$ count and a 1.5625 ns resolution timestamp. The Far Detector timing system uses GPS (Global Positioning System) to provide an absolute pulse per second (PPS) to a TCU (Timing Control Unit), and also to provide date and time to a network time server, housed in the Timing PC (TPC). The TCU sends an echo of the PPS to the TPC, so that the network time server can synchronize the $10 \mathrm{MHz}$ TPC clock with this hardware signal. The network time server then combines the date and coarse time knowledge from GPS with the very precise knowledge of the second from TCU and serves this time to the Read Out Processors (ROPs) which can then timestamp the detector data.

A full account of the details of the Far Detector front end electronics is given in Ref. [65].

Finally, as a means to reduce the cosmic ray background to levels compatible with those necessary for a successful atmospheric neutrino data analysis, a veto shield composed of $8 \mathrm{~m}$ long scintillator modules was deployed along the top and upper side faces of the detector. To further improve background rejection efficiency, the shield is double-layered in the top sections. Apart from higher rates due to the orientation of the modules, shield data collection steps are analogous to the ones already discussed for the regular scintillator planes.

\subsubsection{The Near Detector}

The MINOS Near Detector is located at Fermilab, near Batavia, Illinois, in an undergound hall $100 \mathrm{~m}$ below the surface, $\sim 800 \mathrm{~m}$ downstream of the MINOS target. Its main goal is to measure the unoscillated beam energy spectrum before the neutrinos depart on their journey to Minnesota and beyond. 
The detector has a total mass of 980 ton. It is composed of 282 planes arranged as a squashed octagonal prism, $4.8 \mathrm{~m}$ wide and $3.8 \mathrm{~m}$ in height. The detector pitch is identical to that at the Far Detector. A $40 \mathrm{kA}$-turn magnetic coil is fitted to the detector, running along its axis, but offset $50 \mathrm{~cm}$ horizontally from the center, which represents a distance of $1 \mathrm{~m}$ from the beam spot. In order to ensure the existence of a region with similar properties to the Far Detector, the Near Detector is composed of four different sections. The first one, as traversed by the beam, is the Veto section, which encompassess $0.5 \mathrm{~m}$ of steel. The neutrino interactions in this region are not considered for physics analyses so as to avoid background from neutron events and end effects. The second component is the Target section, containing $1 \mathrm{~m}$ of steel, and the interactions occurring in this region will be the ones used for comparison with the Far Detector. The third one is called the Hadron Shower section, with $1.5 \mathrm{~m}$ of steel, and is long enough to fully contain the showers produced by all the neutrino interactions occurring in the fiducial volume of the Target section. Finally, there is the Muon Spectrometer section, containing $4 \mathrm{~m}$ of steel, responsible for accurately measuring muon momentum either from range or from the curvature in the magnetic field. Again, given the transverse compactness of the beam and the limited transverse spread of hadronic showers in the MINOS detectors, it is not necessary to build a fully instrumented detector. Therefore, in the three upstream regions, 4 out of 5 planes are partially instrumented to a distance of $1 \mathrm{~m}$ out from the beam spot and in the Muon Spectrometer region, only every fifth plane is fully instrumented, still providing accurate curvature measurements for muon tracks. Fig. 2.7 displays an image of the completed Near Detector. Also, due to its smaller size, the Near Detector scintillator strips are read out solely on one side, with the other end of the strip being coated with a piece of reflective aluminized mylar tape to increase light yield. The higher event rates and reduced number of channels with respect to the Far Detector obviate the need for multiplexing. The single side readout uses Hamamatsu M64 PMTs, with 64 pixels, to collect scintillation light. Each PMT anode is read out through a Charge to Current Encoder (QIE) chip, capable of continuous analog processing at $53 \mathrm{MHz}$ without readout dead time. One QIE chip shares a small circuit board, the MENU module, with an ADC and a FIFO for data storage. Sixteen MENU modules 


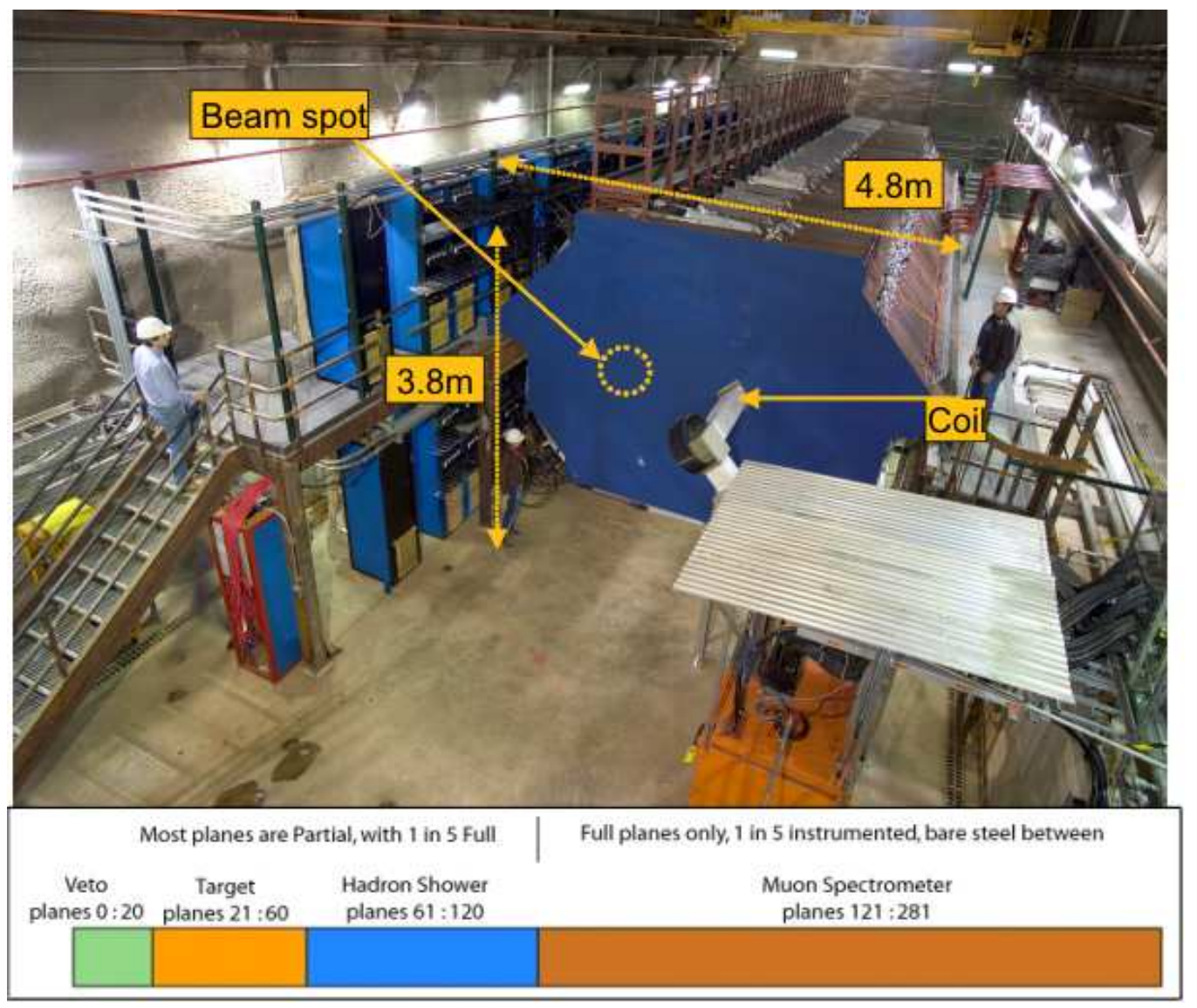

Figure 2.7: The completed MINOS Near Detector. The bottom diagram enumerates the different sections composing the detector. The highlighted beam spot in the upper picture signals where the beam enters the detector and is not a depiction of the beam width.

are integrated into a MINDER module, responsible for applying 19 ns resolution timestamps to the data and providing power, control and interface to the QIEs. The readout charge is thus divided into 19 ns time buckets and each one of them is digitized separately. Reconstruction of these digitizations is analogous to that in the Far Detector.

\subsubsection{Detector Calibration and Response}

The quality of the MINOS oscillation measurement relies heavily on an accurate determination of the neutrino energy. Therefore, careful calibration of the detector 
responses is fundamental.

\section{Charge Injection}

The Far and Near Detectors FEE feature a system with the ability to inject a known amount of charge into the electronic read out cascade, in order to determine the output ADC of the electronic channel as a function of the input charge. This relation is plotted in Fig. 2.8, showing a linear behavior up to about 8000 ADC counts, when the electronics begins to saturate.
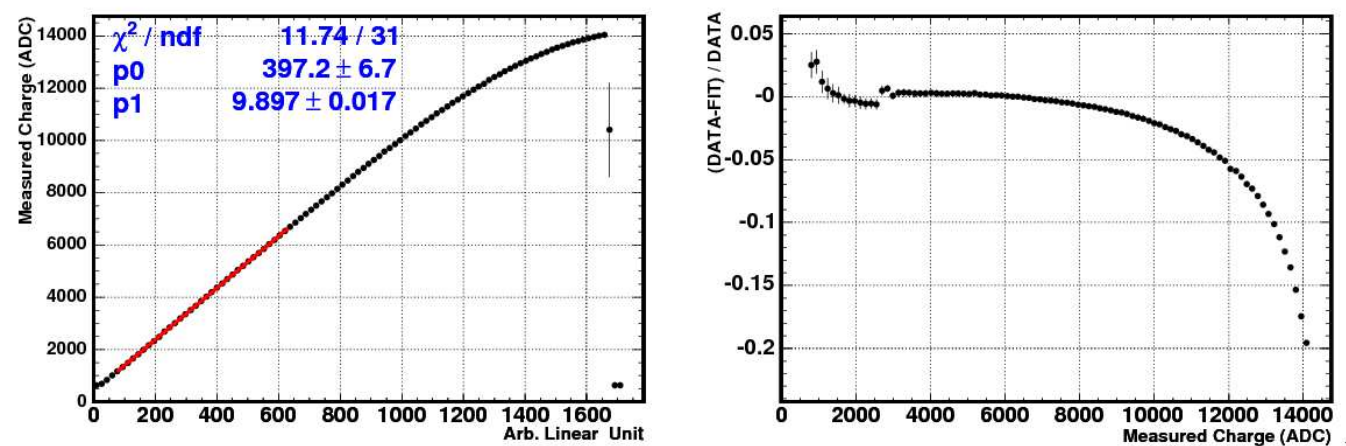

Figure 2.8: The non-linearity of response for a random electronics channel is shown on the left. The residuals shown on the right clearly demonstrate the saturation régime above $8000 \mathrm{ADC}$ counts. The reason for the small non-linearity observed for low ADC counts is undetermined.

In the case of the Far Detector, the charge injection runs are used for corrections of the non-linearity of the PIN diode readout, relevant for light injection calibration runs, as described below. In the Near Detector, the runs are used to generate a lookup table that converts ADC information output and range, from the QIE chip into a linearized ADC.

\section{Light Injection}

The MINOS Light Injection (LI) system measures and compensates for the individual gains of each one of the optical readout channels, identifying drifts in response on a channel by channel basis and linearizing the response of the PMT and electronics. The LI system employs pulsed UV LEDs to inject light in the detector's optical 
path and then compares the output to an independent measure of the light injected. This independent measurement is carried out by a positive-intrinsic-negative (PIN) photodiode that monitors the LED light intensity. Fig. 2.9 shows a diagram of the LI system. Typically, an LI run is performed before a data run starts and the gain

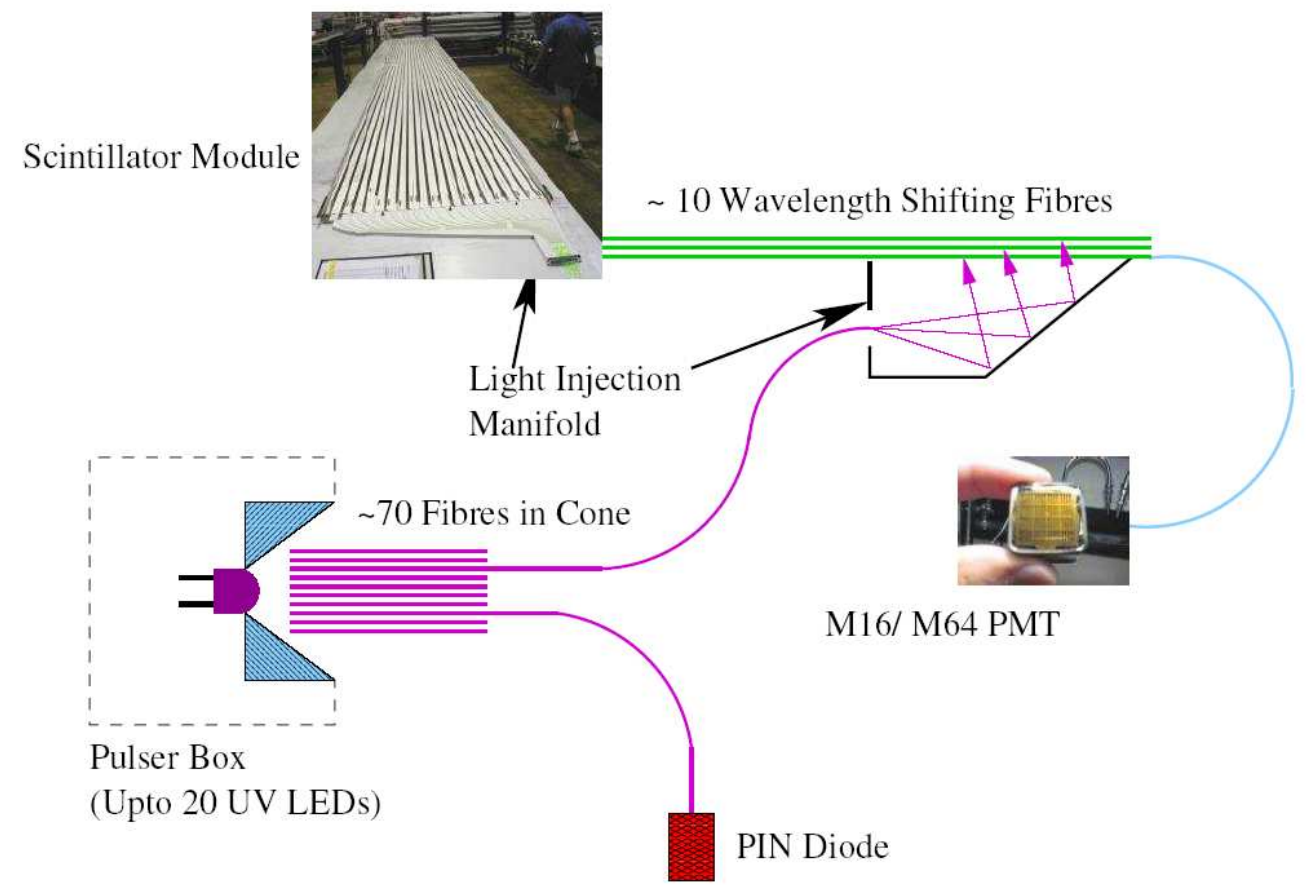

Figure 2.9: Schematics of the MINOS Light Injection calibration system. Obtained from $[66]$.

of each electronics channel is determined at a reference time. Then, special LI runs interspersed with the data taking are performed at 20 minute intervals, comparing the gain at that stage with the reference gains so as to account for any drift. The additional comparison to the PIN response eliminates the drift in light output of the LED with time. Fig. 2.10 shows the evolution with time of the average channel gain as measured by the LI system.

The LI system can also be used to correct for non-linearities in the PMT and electronics readout chain. The MINOS PMTs have a linear relation between input light level and output charge up to $100 \mathrm{PE}$. Above this value, the PMT enters a saturation 


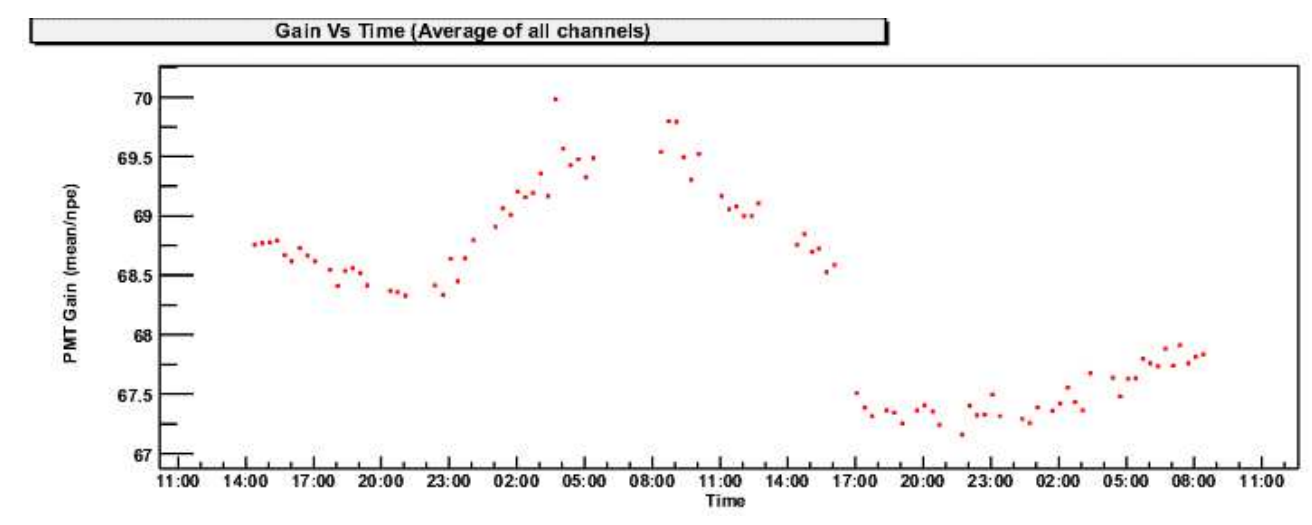

Figure 2.10: Evolution of the average channel gain with time, measured by the Light Injection system.

regime and the output charge flattens. This non-linear behavior is enhanced by the contribution of the electronics readout channels above a certain value, 8000 ADC in the case of the Far Detector. This combined effect can be corrected by pulsing the LI LEDs at different light levels, from 1-200 PE, and comparing the PMT response to the PIN response. The non-linearity on the PIN electronics readout itself can be removed using the charge injection system.

\section{Muon Calibration}

Further calibration of the response of the detectors can be accomplished through the use of muons originating from cosmic ray sources or induced by neutrino interactions. Charge and light injection correct for non-linearities in PMT and electronics, but further non-uniformities result from variations in scintillator strip yield, defects in wavelength shifting fibers and differences in the interfaces between optical elements. One can calibrate these variations by measuring the average light yield of each strip induced by cosmic muon interactions in the detector. A strip-to-strip correction factor can be obtained by normalizing the mean of the pulse height distributions for different strips, as illustrated in Fig. 2.11.

Cosmic muons also play a fundamental role in the relative energy calibration between detectors. The mean pulse height read out from a single strip end when such strip is traversed by cosmic muons is called a MIP, a unit used extensively in data 

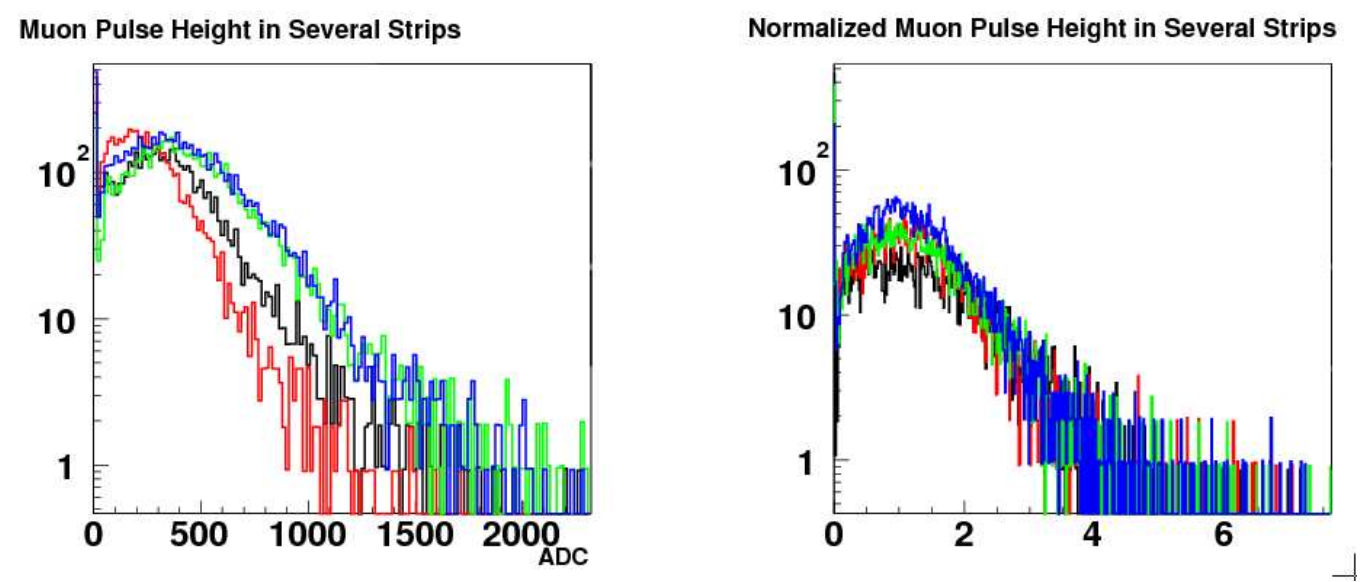

Figure 2.11: Non-calibrated reponse for several detector strips (left-hand side plot) and the normalized response after strip-to-strip calibration corrections (right-hand side plot).

analysis for the Calibration Detector, described below. However, the cosmic muon energy spectrum at each detector location is different due to distinct overburdens and geomagnetic flux effects, so establishing a unit of energy that has the same meaning for all detectors is non-trivial. The universal energy unit to be used by MINOS is named Muon Energy Unit (MEU) and is determined by analyzing the response of stopping muons over a range of planes near the end of the track. Given the similarity between detectors, the stopping muons of a certain track length will deposit almost identical amounts of energy, providing an easy method to perform inter-detector calibrations. Using only the range of planes near the end of a muon track, where $\mathrm{dE} / \mathrm{dx}$ varies little with muon momentum, improves this calibration significantly with respect to using simple range energy measurements, as described in [66]. This reference also defines $1 \mathrm{MEU}$ as the detector response to a perpendicular $1 \mathrm{GeV}$ muon traversing 1 plane of scintillator.

\section{Calibration Detector}

Determination of the absolute energy calibration requires sources of electrons and hadrons of known energy. Given the large size of the Far and Near Detectors and the constraints imposed by their underground locations, exposing them to a calibration 
beam is impractical, if not impossible. In order to obtain an absolute energy scale of the particles interacting in the MINOS detectors, a third detector was built at CERN, Geneva, Switzerland, and exposed to beams of known particles with known momenta.

The Calibration Detector (CalDet) has a mass of 12 tons and is composed of 60 $2.5 \mathrm{~cm}$ thick square steel planes with $1 \mathrm{~m}$ sides. A photograph of the detector is shown in Fig. 2.12. Like the other detectors, each plane also includes a scintillator module,

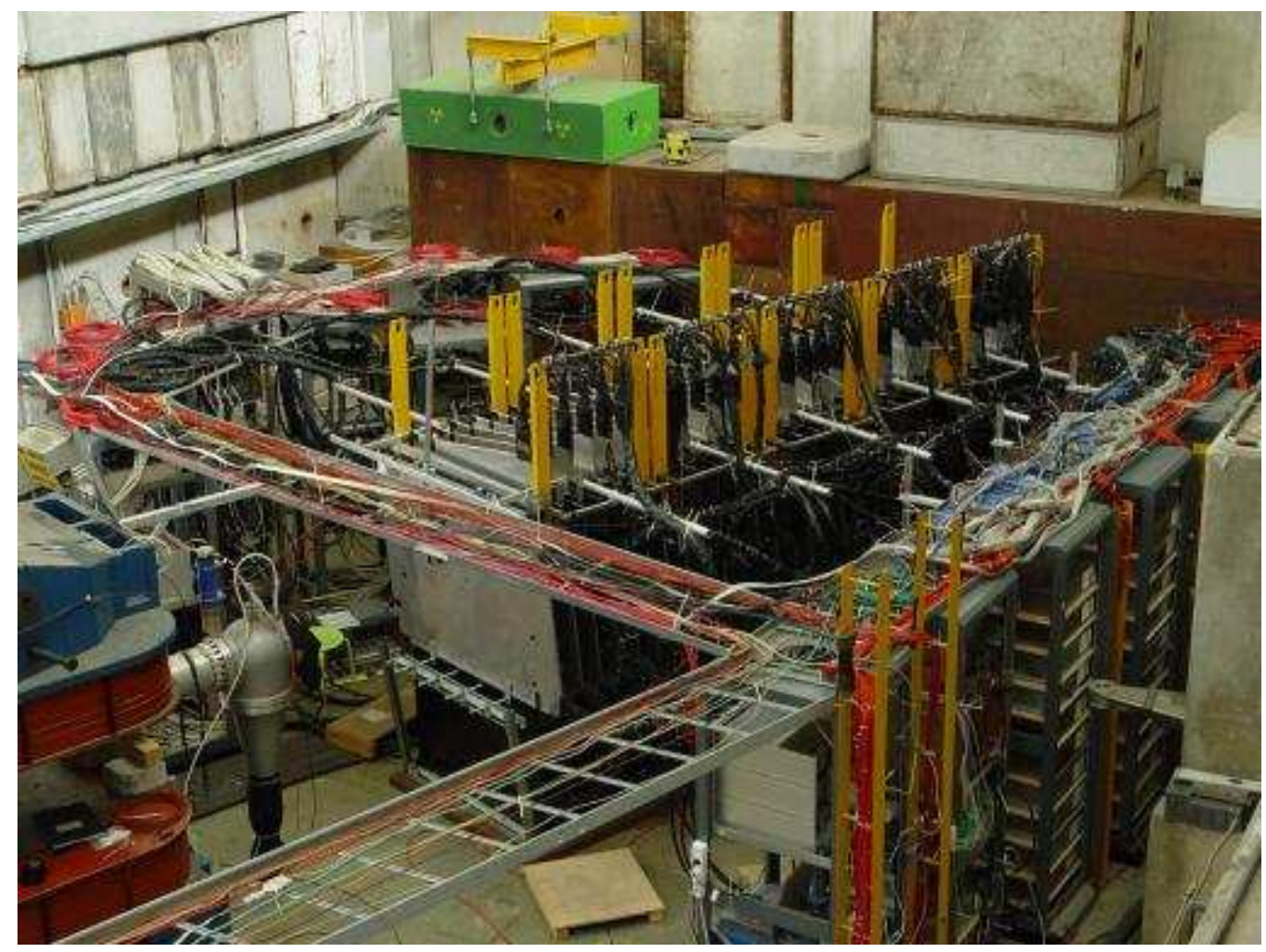

Figure 2.12: A picture of the Calibration Detector in the T7 beamline, assembled at the CERN PS building.

in this case containing 24 strips. The possibility of simultaneous use of Far and Near FEE allows for direct comparisons of the MINOS detector responses. The detector was exposed to test beams delivered by the PS ring at CERN, which were composed of pions, protons and electrons, or muons from pion decay. The test beams used were the T11 and T7 beams, the first capable of delivering particles with momentum in 
the $0.2-3.5 \mathrm{GeV} / \mathrm{c}$ range, the second, in the $0.5-10 \mathrm{GeV} / \mathrm{c}$ range. Through analysis of CalDet data, the measured hadronic shower resolution for the MINOS detectors was found to be [67]:

$$
\begin{aligned}
& \frac{(56.6 \pm 0.6) \%}{\sqrt{E[\mathrm{GeV}]}} \oplus(4.2 \pm 1.4) \% \quad \text { [protons] } \\
& \frac{(56.1 \pm 0.3) \%}{\sqrt{E[\mathrm{GeV}]}} \oplus \quad(2.1 \pm 1.5) \% \quad \text { [pions]. }
\end{aligned}
$$

The electromagnetic shower resolution was measured to be [68]:

$$
\frac{(21.42 \pm 0.06) \%}{\sqrt{E[\mathrm{GeV}]}} \oplus \quad(4.1 \pm 0.2) \% \quad \text { [electrons] }
$$

\subsection{MINOS Physics Reach}

By virtue of the comparison of the observed energy spectrum at the Far Detector with the non-oscillated expectation from measurements at the Near Detector, the MINOS experiment will perform several physics measurements with unprecedented precision. The primary goal of MINOS is to observe the oscillation-induced spectral distortion of the charged current $\nu_{\mu}$ interactions at the Far Detector, i.e. energy dependent disappearance of the beam $\nu_{\mu}$ flux at the Far Detector with respect to the expected spectrum extrapolated from the Near Detector flux. If this dominant oscillation mode is indeed observed, MINOS can:

i) Carry out a direct measurement of the $\mathrm{L} / \mathrm{E}$ dependence of the $\nu_{\mu}$ flux;

ii) Observe the oscillation dip for a neutrino energy of $1.5 \mathrm{GeV}$ if the values of the oscillation parameters $\Delta m^{2}$ and $\sin ^{2} 2 \theta$ fall into Super-K's allowed region of parameter space [69];

iii) Make a 10\% measurement of the oscillation parameters within 2.5 years;

iv) Allow a powerful test of the flavor oscillation mechanism against alternative models such as decoherence or neutrino decay. 

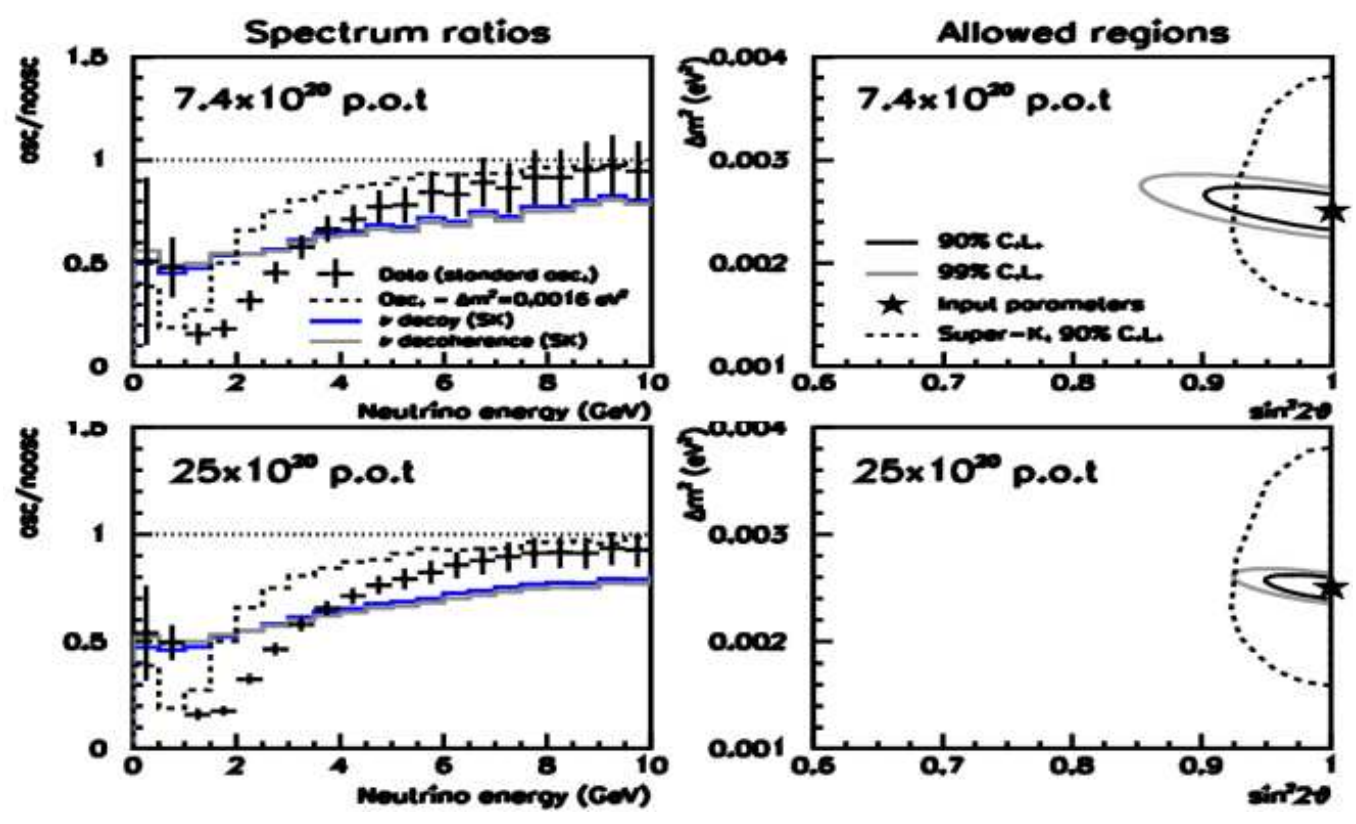

Figure 2.13: The plots above display MINOS ability to measure beam $\nu_{\mu}$ flux disappearance. The two plots on the left show the ratio of the oscillated to the unoscillated spectrum, compared to predictions from alternative models, for different numbers of protons incident on the NuMI target. The oscillation parameters used are $\Delta m^{2}=2.5 \times 10^{-3} \mathrm{eV}^{2}$ and $\sin ^{2} 2 \theta=1$. To the right, the plots show the allowed regions in the oscillation parameter space. Even for a relatively small number of protons on target, MINOS can significantly improve the current limits on $\Delta m^{2}$.

Fig. 2.13 illustrates these MINOS capabilities.

MINOS can also perform an independent statistical test of the existence of oscillations by computing the ratio of charged current $\nu_{\mu}$ events (typically long events, with a small background from $\nu_{\tau}+\mathrm{N} \rightarrow \tau+\mathrm{N}, \tau \rightarrow \mu+2 \nu, E_{\nu_{\text {beam }}}>3.5 \mathrm{GeV}$ ) to all events in both the Near and Far detectors. The ratio of ratios $R_{\text {Near }} / R_{\text {Far }}$, also referred to as T-test, is independent of beam systematic effects, as they cancel out to first order in the ratios, and will indicate existence of $\nu_{\mu} \rightarrow \nu_{x}$ oscillations if its value is a considerable departure from unity. In addition, if one can successfully separate short charged current events from neutral current events, the ratio $\left(\frac{C C}{N C}\right)_{N e a r} /\left(\frac{C C}{N C}\right)_{F a r}$ could provide discrimination between $\nu_{\mu} \rightarrow \nu_{\tau}$ and $\nu_{\mu} \rightarrow \nu_{\text {sterile }}$ oscillation modes.

MINOS is also able to conduct appearance searches, in particular the observation of the small signal induced by $\nu_{\mu} \rightarrow \nu_{e}$ sub-dominant oscillations, by measuring an 
excess of charged current $\nu_{e}$ interactions at the Far Detector with respect to the extrapolated Near Detector measurement. Any observed $\nu_{e}$ events at the Near Detector will originate solely from the small $\nu_{e}$ component of the NuMI beam. Fig. 2.14 shows the official representation of the parameter space for which MINOS can observe $\nu_{e}$ appearance, originating from early Monte Carlo studies [70]. As can be seen, MINOS can potentially improve on the current best limit set by the region excluded by the CHOOZ reactor experiment. Identifying the current sensitivity and extent of the
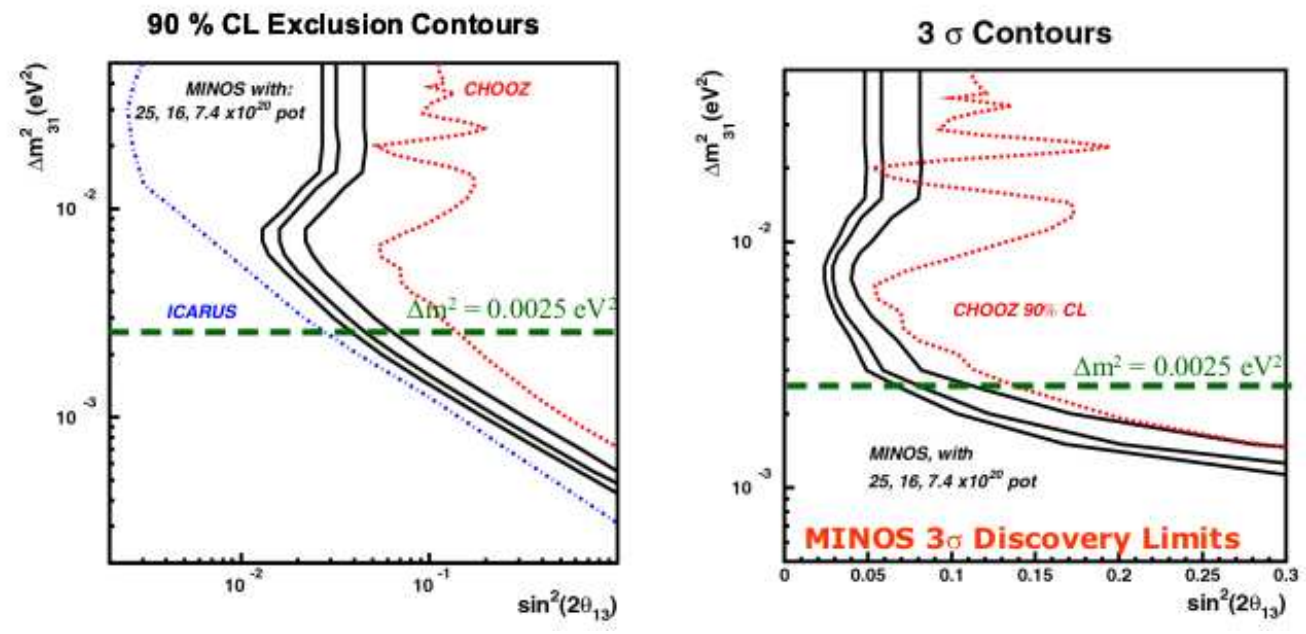

Figure 2.14: The plot on the left illustrates MINOS sensitivity to $\nu_{\mu} \rightarrow \nu_{e}$ appearance compared to CHOOZ and ICARUS. The right-hand side plot represents the allowed region contours assuming $\Delta m^{2}=2.5 \times 10^{-3} \mathrm{eV}^{2}$ and $\sin ^{2} 2 \theta=1$. The CHOOZ limit is shown for reference.

capabilities of MINOS concerning this measurement are the object of this thesis and will be discussed in detail in the following chapters.

The MINOS experiment operates the first ever large magnetized detector deployed underground. By inversion of the magnetic field in the beam parabolic horns and also of the fields generated by the Far and Near detector coils, MINOS can focus $\bar{\nu}_{\mu}$ in identical fashion to the regular $\nu_{\mu}$ focusing. Therefore, MINOS can compare the oscillation properties of neutrinos and anti-neutrinos and test CPT violation models. Charge separation of atmospheric neutrinos will also provide a test of CPT violation and in turn, place bounds on existing Lorentz-invariance violating models. MINOS 
sensitivy to a CPT violation measurement using atmospheric neutrinos is shown in Fig. 2.15.

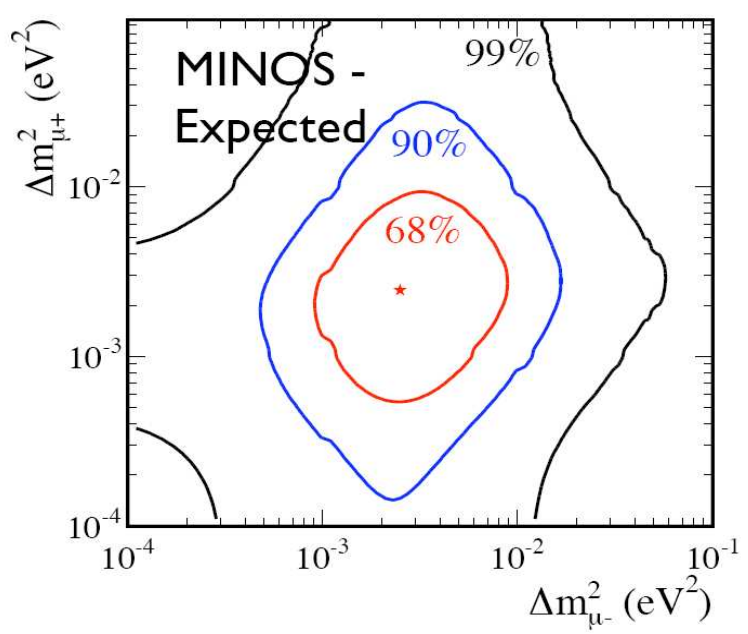

Figure 2.15: CPT violation 68\%, $90 \%$ and $99 \%$ allowed parameter regions after 4 years of atmospheric neutrino data taking.

\subsection{MINOS Atmospheric and Beam Neutrino Data}

During the Far Detector construction phase, from 2001 to 2003, the modular approach used in erecting the planes and connecting clear fiber cables and the necessary electronics, allowed for continuous data taking of both cosmic muon data and atmospheric neutrino data. After the completion of construction in July 2003, the Far Detector continued to accumulate atmospheric data in the same way and it will do so for the duration of the experiment. These data are being used in studies of neutrino oscillations via measurements of upward-going neutrino induced muons and of fully and partially contained atmospheric neutrino induced events. The total sample accumulated so far consists of 418 live days of running, corresponding to a total exposure of 6.18 kton-year, 4.54 kton-year fiducial. 91 upward going and 107 fully contained event candidates have been identified. Fig. 2.16 illustrates MINOS $1 / \beta$ distribution for cosmic ray muons and shows a preliminary zenith angle distribution obtained from the atmospheric neutrino data. 

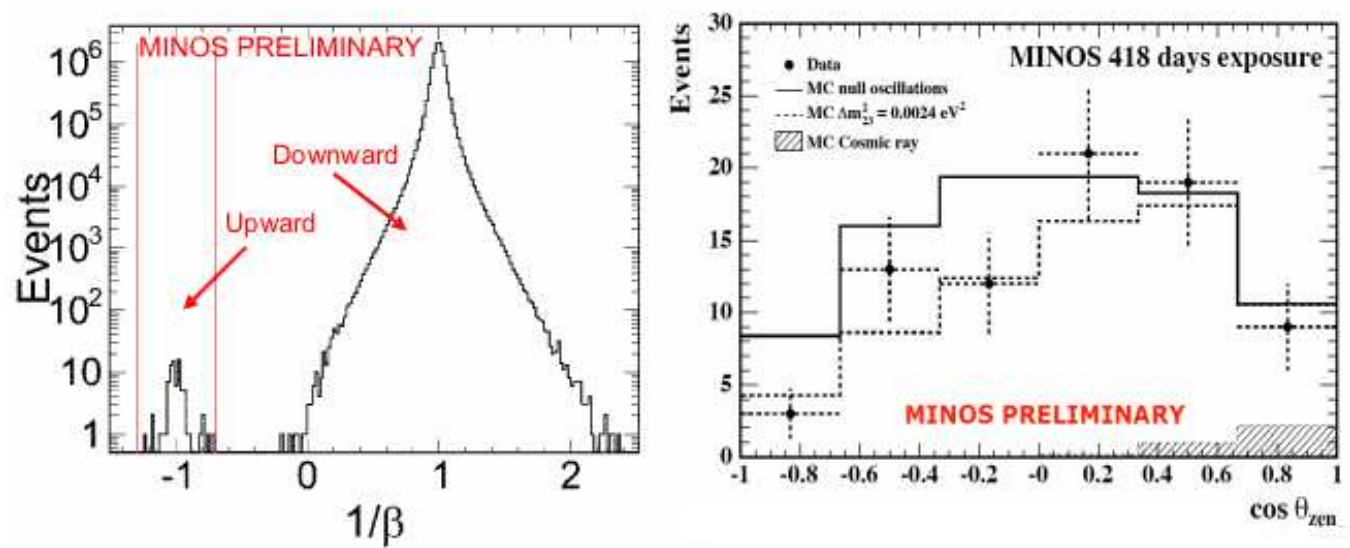

Figure 2.16: Cosmic ray muons $1 / \beta$ distribution for a 6.18 kton-year exposure is displayed on the left. Zenith angle distribution for data compared to oscillated and unoscillated Monte Carlo is shown on the right.

The ratio of ratios obtained from comparison of the up-down ratio for the fully contained data with its corresponding Monte Carlo value in the absence of oscillations is

$$
R_{\text {up } / \text { down }}^{\text {data }} / R_{\text {up } / \text { down }}^{M C}=0.62 \pm 0.14(\text { stat. }) \pm 0.02(\text { syst. })
$$

which already excludes a no oscillations result at the level of $2.6 \sigma$.

At the time of writing of this thesis, MINOS is regularly taking beam data. The first Main Injector protons reached the target hall in December 2004 and the first beam induced neutrino events were seen in the Near Detector in January 2005, coinciding with the commissioning of the NuMI beam. The first neutrino interaction observed in the Far Detector consistent with the beam spill trigger window occurred in late February 2005. As of December 2005, MINOS has collected a total beam data sample corresponding to an intensity of $1.0 \times 10^{20}$ POT. The running intensity is about $2.5 \times 10^{13}$ POT for every $1.9 \mathrm{~s}$ Main Injector cycle. The evolution of MINOS beam data collection through July 2005 is summarized in Fig. 2.17. 


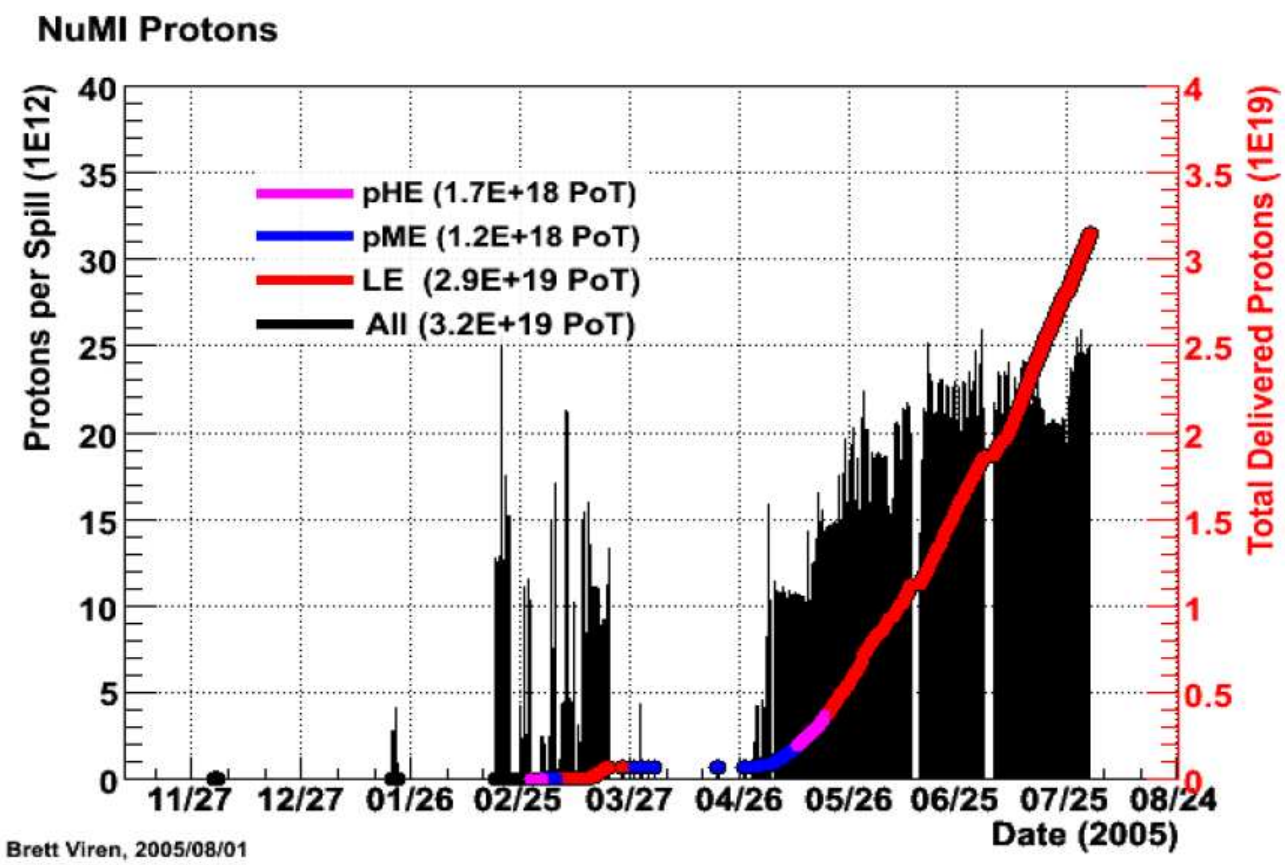

Figure 2.17: The evolution of MINOS proton intensity since the NuMI beam commissioning. Different types of beam data runs are displayed, corresponding to different target-horns configurations. The empty region in April corresponds to downtime due to repairs of leakage in the target cooling system.

\subsection{MINOS Monte Carlo Data}

In order to quantify an experiment's sensitivity to a physics measurement, as well as to compare that measurement to theoretical models, it is necessary to generate simulated data describing as accurately as possible the physical processes involved in the experiment framework. MINOS uses the Monte Carlo method to generate such data. With MINOS being a multi-detector, beam based experiment, creation of Monte Carlo events is a complex task. It entails simulating the entire NuMI neutrino beam line, creating neutrinos with random parameters, conceiving electronic versions of both detectors, simulating the neutrino interactions inside the detectors and finally simulating the transformation of the energy deposition in the detector into light and electronic responses, which can then be reconstructed in identical fashion to the real data. The full Monte Carlo simulation of MINOS data consists of the following 
elements:

\section{GNUMI}

A GEANT-3 based simulation of the NuMI neutrino beam line. The simulation of hadron production leading to the beam neutrino flux that will enter the detectors is performed using the FLUKA code, a generic purpose tool for calculations of particle transport and interactions with matter, developed at CERN and INFN Milano $[71][72]$.

\section{NEUGEN}

A neutrino event generator that simulates neutrino-nucleus interactions over the energy range of $100 \mathrm{MeV}$ to $100 \mathrm{GeV}$. It uses a pre-tabulated library of neutrino cross sections to speed up execution [73]. It originated with the Soudan 2 collaboration and its continued development within Soudan 2 and MINOS has been led by H. Gallagher at Tufts.

\section{GMINOS}

A GEANT-3 based simulation of the detectors developed at SLAC and Fermilab that also acts as a framework to select neutrino fluxes from different methods, call event generators, configure the detector geometry and pass events to GEANT-3 for tracking, recording truth hits, and storing the whole set of event parameters into ADAMO (ALEPH DAta MOdel) data structures, also designed at CERN. GMINOS also uses the GCALOR package to simulate hadron interactions in the detector. The configuration of a GMINOS job is done through an FFREAD (Free Format input processing) file or card. The generated data structure is stored in a GAF (Generic ADAMO File) file. The three components described so far are all written in FORTRAN and form the MINOS software package known as the Labyrinth [74].

\section{PhotonTransport and DetSim}

These two packages are both written in Object Oriented $\mathrm{C}++$ programing language and are based on the ROOT framework. PhotonTransport reads the data structures 
generated by GMINOS, previously converted to the ROOT data format, simulates how energy depositions in a detector are transformed into light and how that light is transported to the PMT photocathodes and converted to photo-electrons. DetSim simulates the MINOS front end instrumentation, carrying the PhotonTransport signals through the PMTs, electronics and data acquisition system. The final output is an object denominated RawDigitDataBlock, which can be processed through the MINOS Offline reconstruction chain in the same way as a real data event.

Many different types of MINOS Monte Carlo events have to be generated for analysis purposes. In the research work described in this thesis, we will be focusing on the Monte Carlo sample generated within the context of the MINOS Mock Data Challenge, to be discussed in the following chapter. 


\section{Chapter 3}

\section{The Mock Data Challenge}

During the January 2004 MINOS meeting at Cambridge, the spokesmen issued the Mock Data Challenge (henceforth referred to as MDC) to the collaboration. The underlying idea consists of generating a Monte Carlo neutrino sample with some oscillation parameters mimicking the expected real beam data, hence the Mock Data designation. The oscillation parameters used are hidden from the collaboration and known only by an MDC Truth committee. The MDC sample allows the collaboration to ascertain the readiness and quality of the work developed by the different analysis groups, uncovering the main weaknesses and flaws requiring more urgent focus, prior to the beginning of beam data collection. The MINOS Mock Data Challenge served the following purposes [75]:

1) Consolidation of the best knowledge of physics models into the data simulations.

2) Generation of a uniform and consistent set of data files that can be used in a multitude of analyses.

3) Production of files to exercise the full analysis, thereby testing the overall software framework and data handling, and testing both the Near detector and Far detector reconstruction chains.

4) Challenging the analysis groups into extracting results, especially for the $\nu_{\mu} \rightarrow \nu_{x}$ disappearance oscillation signal, but also for the possible $\nu_{\mu} \rightarrow \nu_{e}$ appearance and $\nu_{\mu} \rightarrow \nu_{\text {sterile }}$ signals. 
The MDC does not, however, serve as a test of the MINOS calibration chain, since no calibration parameters were varied in the challenge set and the same calibration constants were used in the reconstruction of the whole sample.

The research work described in this thesis was developed within the context of the Mock Data Challenge and used the MDC sample. Comparison of the results of this analysis with the true parameters used to generate the MDC sample, revealed in the 2005 Week-in-the-Woods Collaboration meeting in Ely, are presented in Chapter 5.

This chapter describes the details of generation and reconstruction of the MDC sample along with a characterization of the framework developed by the $\nu_{\mu} \rightarrow \nu_{e}$ analysis group. Emphasis is given to the data reconstruction developed by the author that is integrated into that framework.

\subsection{Event Samples and Generation}

The MDC sample is divided in two sets, a Monte Carlo (MC) set and a Challenge set. The MC set includes Near and Far detector files and the truth information is made available to the analysis groups. The MC Far set is unoscillated. A number of auxiliary Far MC files, where all the $\nu_{\mu}$-induced events have been converted into $\nu_{e}$ or $\nu_{\tau}$-induced events, is available in parallel to the nominal beam files . The Challenge set also includes Near and Far detector files, and is available in two exposures, one corresponding to 2.5 years of running and the other to 7.5 years, the latter allowing for a test of analysis results which are thus mostly limited by systematic errors. In the case of the Challenge set, the truth information is withheld from public access during the reconstruction stage and does not incorporate the reconstruction ntuples made available to the Collaboration. The Challenge Far files are oscillated with secret parameters. A break down of the files composing the MDC sample is presented in Table 3.1.

The generation of the MDC sample was done using GNUMI version 16. The energy and probability for each event were re-weighted according to the position in the detector face. In addition, the challenge set underwent re-weighting of the hadronic production model. NEUGEN3 was used to simulate the neutrino-nucleus 


\begin{tabular}{|c|c|c|c|c|}
\hline MDC Samples & Beam & $\nu_{e}$ flavor & $\nu_{\tau}$ flavor & POT/file \\
\hline \hline Far MC & 40 & 39 & 39 & $6.5 \times 10^{20}$ \\
Far Challenge & 3 & - & - & $7.4 \times 10^{20}$ \\
\hline Near MC & 229 & - & - & $1.33 \times 10^{16}$ \\
Near Challenge & 246 & - & - & $1.33 \times 10^{16}$ \\
\hline
\end{tabular}

Table 3.1: Break down of the MDC sample. The number of files in each simulation set is shown along with the corresponding number of protons-on-target contained in each file.

interactions. The following parameters were used for the MC set:

1) Quasi-elastic axial-vector mass QEL_MA.

2) Resonance production axial-vector mass RES_MA.

3) Deep Inelastic Scattering fudge factor KNO.

The Challenge data uses the same physics models, but with modified unknown values for these parameters. Concerning the oscillation parameters, the set used for the Far Challenge sample was randomly selected according to the conditions:

1) $100 \%$ Probability of existence of $\nu_{\mu} \rightarrow \nu_{\tau}$ oscillations:

i) $\log _{10}\left(\Delta m_{23}^{2}\right)$ selected from gaussian(SK value, $\left.1.5 \times 1 \sigma_{S K}\right)$.

ii) $\sin ^{2}\left(2 \theta_{23}\right)$ selected from gaussian(SK value, $\left.1.5 \times 1 \sigma_{S K}\right)$ restricted to the physical region.

2) $75 \%$ probability of existence of $\nu_{\mu} \rightarrow \nu_{e}$ oscillations:

i) parameters selected uniformly up to $\mathrm{CHOOZ} \mathrm{95 \%} \mathrm{confidence} \mathrm{level} \mathrm{limit.}$

3) $75 \%$ probability of existence of $\nu_{\mu} \rightarrow \nu_{\text {sterile }}$ oscillations.

i) parameters selected uniformly up to SK $95 \%$ confidence level limit.

4) No CPT violation, decoherence, Lorentz invariance violation, $\nu$ decay, LSND or oscillations with matter effects were included. 

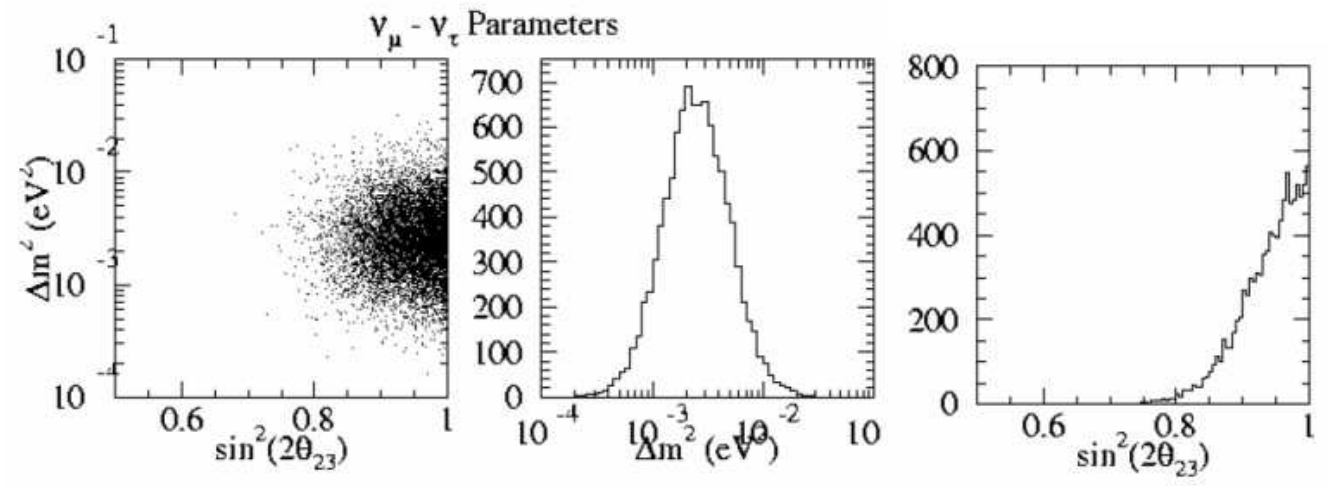

Figure 3.1: Values selected for $\nu_{\mu} \rightarrow \nu_{\tau}$ MDC oscillation parameters for several random test runs. The center and right-hand side plots show the gaussian probability distributions for $\Delta m^{2}$ and $\sin ^{2} 2 \theta$ parameters, centered around SuperKamiokande's best fit values (see Ref. [75] and references therein).

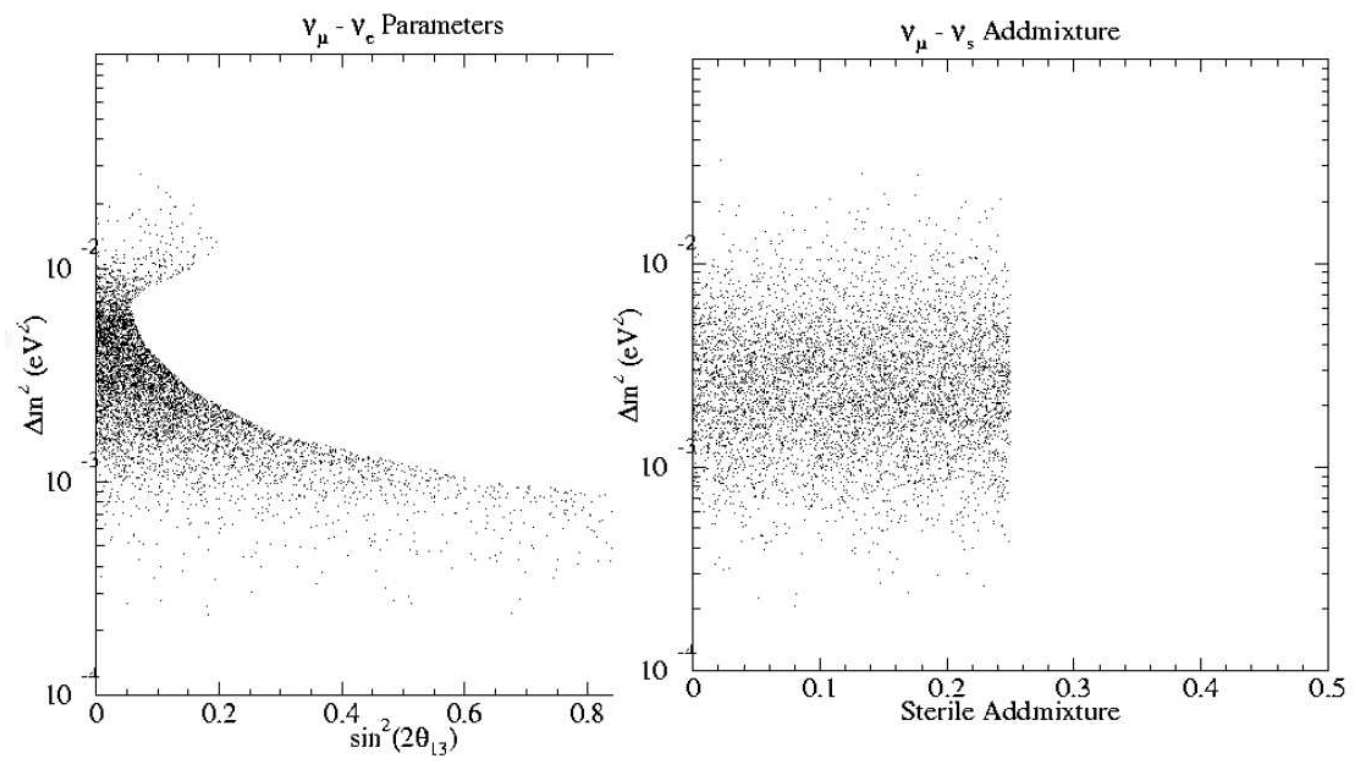

Figure 3.2: The values selected for the $\nu_{\mu} \rightarrow \nu_{e}$ (left plot) and $\nu_{\mu} \rightarrow \nu_{\text {sterile }}$ (right plot) MDC oscillation parameters for several random test runs. The parameter values are selected uniformly up to CHOOZ 95\% CL and SK 95\% CL respectively (see Ref. [75]).

Fig. 3.1 and Fig. 3.2 show the possible values for the oscillation parameters and the most probable regions of value selection.

The generation of such a large number of files as that required by the MDC 
dictated the need for substantial computing resources. It was therefore decided to split production between single machines at Fermilab, Tufts University and Indiana University, using a statically linked GMINOS executable assembled by Dr. R. Hatcher. The combined generation capacity of the 5 machines was still severely inadequate, in particular in the case of the Near sets. A total generation time of 1.5 months was estimated. The author investigated the possibility of using the recently created Tufts Linux Research Cluster facility to expedite this generation. This facility comprises 32 dual-CPU PIV-2.6GHz nodes, interconnected with a Gigabit network, that allows for submission of 64 jobs running in parallel, via the LSF submission system. A 2 TB storage space quota was secured for the Physics Department. The use of this stateof-the-art resource permitted the generation of the remaining $80 \%$ of the total MDC sample in 9 days. The generation working model used at the Tufts Cluster consists of the following phases (see Section 2.5 for details on MC components):

1) Preliminary creation of rock muon FFREAD cards and generation of the corresponding rock muon files for Near detector event overlaying (1 week/file).

2) Creation of FFREAD cards for single MC file generation (containing 1650 snarls), seeded by run number.

3) Using GMINOS to generate the GAF files ( $\sim 15$ hours/file).

4) Overlaying of each GAF file with a rock muon file ( $\sim 1.5$ hours/file). Due to the lengthy generation of rock muon files, each one is used to overlay 6 single files.

5) Splitting of each overlaid file into three 550 snarl files.

6) Conversion of the ADAMO data structures into ROOT trees (rerooting) of the split files $(\sim 0.5$ hours/file).

7) Tranferring of the reroot files to Fermilab's mass storage, a tape archive accessed through the ENSTORE interface.

This working model served as a prototype that allowed other MINOS institutions to join the Off-Site MC Generation effort, namely the College of William \& Mary. 
The post-MDC Monte Carlo generation has been assured in almost its entirety by Tufts University and the College of William \& Mary. The files stored at Fermilab have to undergo reconstruction before being made available to the collaboration and the analysis groups. The intricacies of this process are described in the next section.

\subsection{Data Reduction}

The data produced by different readout processes at the MINOS detectors is regularly sent to and stored at Fermilab's tape robot by a dispatcher process. The diverse data streams written to tape include physics event data, pulser calibration data, beam monitoring data and detector control data. The relevant streams are transferred to Fermilab's fixed target farm and subsequently undergo reconstruction using the MINOS Offline Software. The reconstructed ntuples, containing many useful quantities calculated from the raw data structures, are in turn stored back into the tape robot, where they can be picked up by the collaboration at large for further analysis.

The MINOS Offline software (Minossoft) is almost exclusively written in objectoriented $\mathrm{C}++$ and makes extensive use of the ROOT system, an object-oriented data analysis framework developed at CERN. Minossoft comprises numerous packages, able to reconstruct both raw and simulated data, perform data calibration, provide tools for access to and navigation of MINOS databases, and carry out specific types of data analysis. Minossoft is distributed throughout the Collaboration using CVS (Concurrent Versioning System) embedded in the SLAC-Fermilab SRT (Software Release Tools) code management system. At more or less regular periods, the main development trunk of the code (also known as development release) is branched into what is called a "frozen" release. These stable releases are numerically tagged and are guaranteed to compile. Some of these releases are adopted for production event reconstruction and submitted to thorough testing at the batch processing farm. The current release used at the batch farm for data reconstruction is R1.18. The processing of the MDC sample resulting in the ntuples used for the research work described in this thesis used the production frozen release R1.12. 


\subsubsection{Batch Processing}

The MINOS batch processing group was formed in June 2003 with the purpose of standardizing and automating as much as possible the reconstruction of a fast growing volume of collected data, which at that time was mostly cosmic ray data collected with the partially built Far detector. The prototype working model for batch reconstruction of MINOS data was designed, implemented and tested by the author and Prof. Howard Rubin, of the Illinois Institute of Technology. It uses 100 CPUs from Fermilab's fixed target computer farm and a locally installed MINOS MySQL database along with multiple Minossoft releases. The job submissions proceed through FBSNG (Farm Batch System Next Generation), the software responsible for scheduling job running on a given queue depending on CPU load and user priority. The steps involved in the production reconstruction of a data file are as follows:

1) A list of files to be processed is automatically generated by a preliminary job that runs a quality check on recently transferred files, eliminating the ones where data collection occurred during high voltage failures, magnet trips, etc..

2) A daily cronjob ${ }^{1}$ running on the batch farm head node picks up the list and submits the file numbers therein for processing using FBSNG.

3) In parallel, another cronjob updates the farm database every 8 hours with the latest beam monitoring and spill time information.

4) The submitted jobs are divided in three sections, input, analysis and output. During the input phase, the data file corresponding to a run number in the list is requested from ENSTORE and transferred to the working node.

5) In the analysis phase, a standard reconstruction script is used jointly with Minossoft to produce ntuples containing several useful quantities calculated from the raw data. At the end of the analysis phase a quality monitoring job runs on the output, gauging whether reconstruction was successful.

\footnotetext{
${ }^{1}$ UNIX command or script executed periodically at a time defined by the user.
} 
6) If the quality monitoring job yields successful results, the run enters the output phase, where the candidate, standard and abridged ntuples are transferred to dfarm, a large hard-disk based storage resource.

7) A cronjob archives the newly available ntuples from $d f a r m$ into the tape robot.

For Monte Carlo files, as in the case of the MDC sample, step 1) only involves generating the list of files to be run and is done manually. A diagram of the batch processing model is shown in Fig. 3.3. The tapes allocated for MINOS use are divided into different families and each family corresponds to a type of input file and/or reconstruction. This allows for efficient recycling of tapes holding obsolete reconstruction output. Since its inception, the MINOS batch farm production system has processed and archived a total of over 7 TB of data. Since September 2003, this processing has progressed into a fully-automated, continuous-running mode, with no human intervention from the time the data leaves the Soudan mine or the Near detector hall, to the time the reconstructed data is stored at Fermilab. The thorough testing necessary before the start of production runs combined with the large variety and number of data runs processed allows the uncovering of many inconspicuous and infrequently occurring problems in the MINOS offline software. The batch processing group has thus been one of the main driving forces behind several quality improvements undergone by Minossoft in recent years. Fig. 3.4 depicts a summary plot of the weekly usage of the fixed target farm, showing the share corresponding to MINOS activity. 


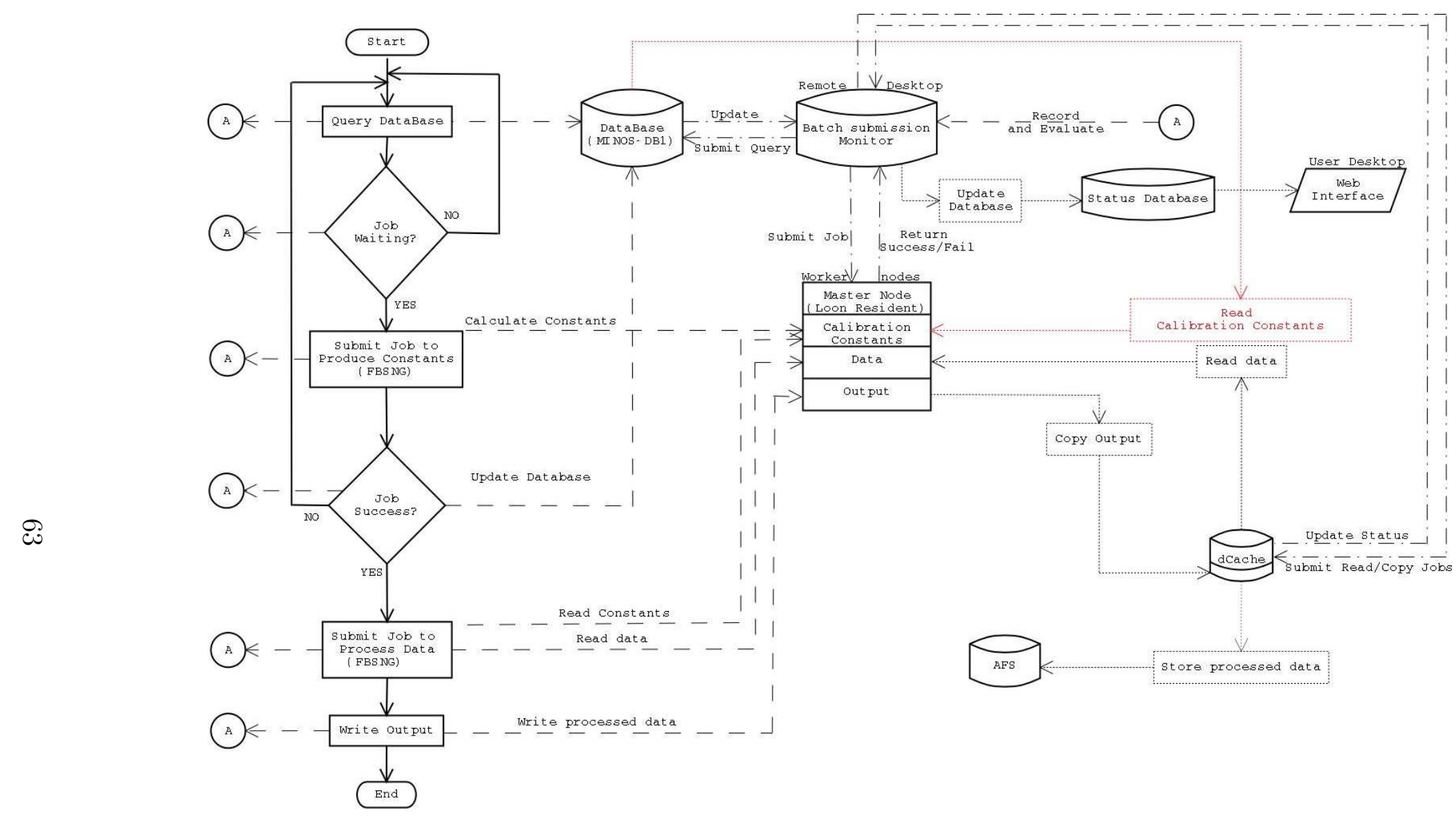

Figure 3.3: A diagrammatic view of the MINOS Batch Processing system designed and implemented by Prof. H. Rubin and the author. 


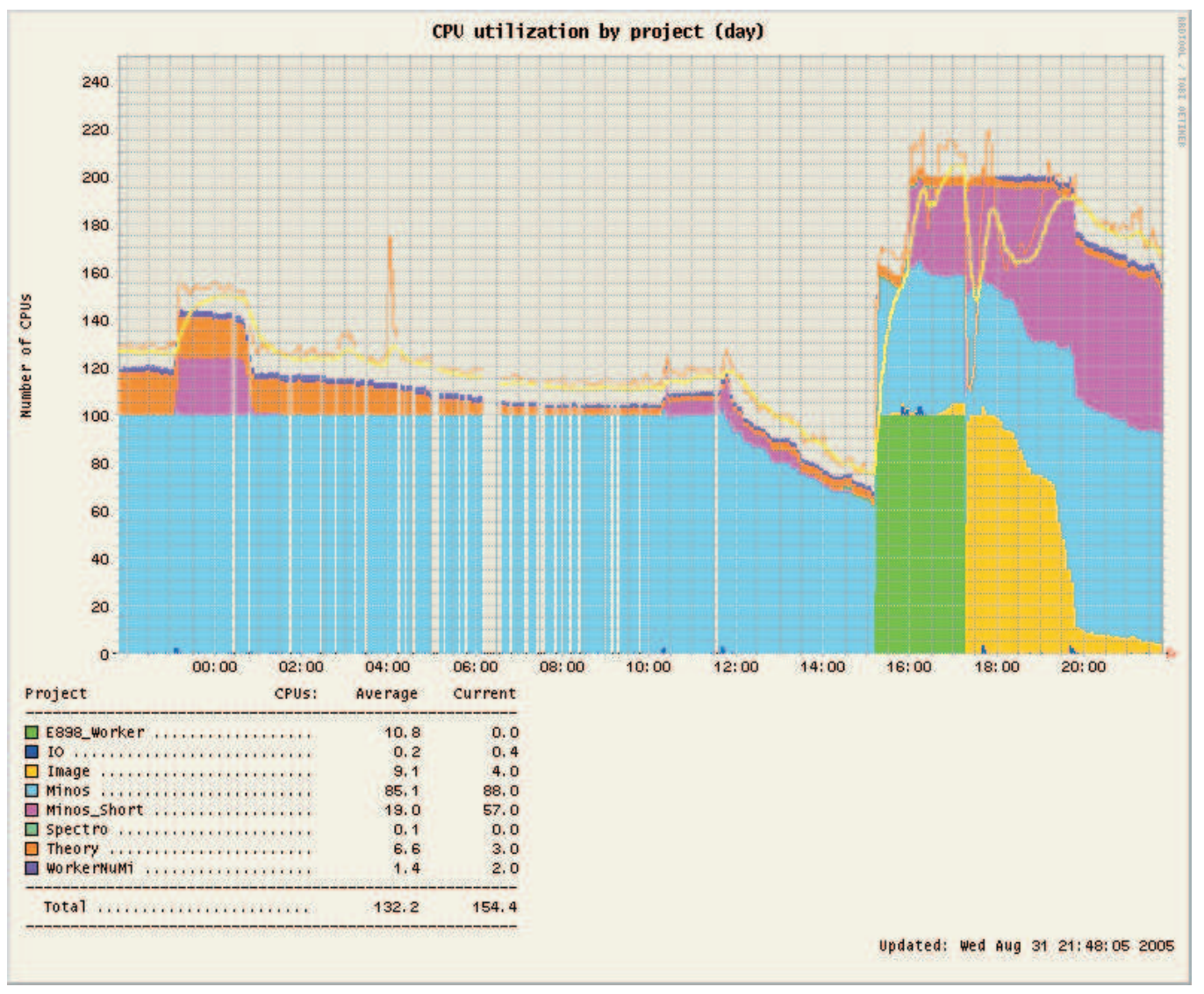

Figure 3.4: This FBSNG farm monitoring plot shows the number of CPUs occupied by each queue during a single day period. The average MINOS share for this particular day was 88 CPUs out of a possible maximum number of $100 \mathrm{CPUs}$ allocated to the queue.

\subsubsection{Reconstruction}

The reconstruction of MINOS data involves a large number of software packages. The configuration of a reconstruction job is done through JobControl scripts or macros. These macros select which software modules to run, in which order, and allow the user to set running parameters to each module. The JobControl package, also part of Minossoft, interprets the contents of the macros and passes the appropriate options to the Minossoft executable, dubbed loon by the collaboration. A typical reconstruction script, such as the ones used in the analysis stages of batch processing, comprises the 
following elements:

1) Loading of all the dynamical libraries corresponding to packages to be used by the loon executable.

2) Definition of the reconstruction path, which calls each of the packages or modules to be run in the proper sequential order, and must include the relevant components:

a) In the case of MC or Mock Data files, an input module reads the reroot file and extracts the truth information. During this stage, as mentioned in Section 2.5, the DetSim and PhotonTransport packages are used to simulate PMT and electronics response so that digitizations similar to those in the data are available for the following components of the reconstruction path.

b) A noise filter module is applied to remove spills or snarls containing an uncorrelated low number of digits with a low total amount of energy deposited $(<20 \mathrm{PE})$.

c) A module that creates lists of candidate digits from the raw data to serve as input to the remainder of the reconstruction.

d) In the case of Far detector data, a demultiplexing algorithm is applied, picking from the candidate digit list the best hypothesis for the combination of strips that form each snarl. The differentiation and selection among the 8 possible hypotheses is achieved through comparison of the East and West readouts for that particular snarl (see Ref. [76] and Ref. [77]).

e) From the demuxing output, another module creates a list of strips per snarl.

f) The list of strips is fed into a slicer module, which identifies possible multiple interactions within the same time bucket, which happens often at the high intensity environment that characterizes the Near detector.

g) In each slice, density regions are identified by a clustering module.

h) The clusters thus obtained are identified as showers and written to a list. In the case of R1.12 processing, no further reconstruction was applied to showers. 
i) A Kalman filter algorithm simultaneously finds and fits tracks in the strip information, writing the found tracks to their own list. For each track, the momentum is determined from both range and curvature. Further details on the Standard Reconstruction of tracks and showers in MINOS are available in Ref. [78].

j) The reconstruction results, along with relevant quantities calculated from them and truth info when available, are written into a ROOT data structure, which we will refer to as the Standard ntuple.

k) An output module writes out the Candidate ntuple, which, besides the reconstruction quantities, includes the original digits.

3) Configuration of the modules contained in the reconstruction path, such as choice of demuxing algorithms for beam or cosmic-ray data, choice of track fitting algorithm for Near or Far detector data reconstruction, etc..

4) Configuration of the Message Service, defining the verbosity of the job output, for either debugging or simple monitoring of job progress.

5) Job execution. The job may be configured to run the reconstruction on a selected number of snarls in the data file or to run on a snarl by snarl basis, useful for event displaying purposes.

It should be noted that, if any package used in the reconstruction script requires database information retrieval, a loon job will start by accessing the central or a local copy of the MINOS database and load all the necessary tables. For reference, all the macros used in the Mock Data Challenge reconstruction, assembled by the author and vetted by the MINOS collaboration, are presented in Appendix A.

\subsubsection{Reconstruction ntuples}

The reconstruction jobs output three types of ntuples. The first one, the Candidate output (identified by the affix "cand" in the name of the output file), contains the original digits, reconstructed quantities and truth information in the case of MC 
files. It requires the use of the loon executable and the size of each file, typically 220 MB for a Far Detector file, makes their usage cumbersome. The Candidate files are valuable when one wishes to run simplified reconstruction paths, e.g. by running a single module in the reconstruction path, with a custom configuration, avoiding rerunning the full reconstruction job.

The second output is the Standard ntuple (identified by the affix "sntp" in the name of the output file). It contains the complete strip information for each event combined with reconstruction variables and truth information for MC files. It consists of a multi-branch ROOT TTree data structure and so it can be read and used in a simple ROOT session. The ntuple size is approximately twenty times smaller than the Candidate output size, making it more manageable for analysis purposes. With a separate interface - such as the one implemented by the NueAna package, described in the next section - the Standard ntuples can also be used within the context of the Minossoft framework. In this way, Minossoft tools can be utilized to access the validity context, geometry and beam monitoring information provided by the MINOS database.

Finally, the third output consists of the Short ntuples (identified by the affix "snts"), which are essentially Standard ntuples divested of the strip information. These ntuples have the smallest size, four times smaller than Standard ntuples and can be more easily distributed to the collaboration. They are useful for quick data analysis where no alternative reconstruction methods are employed and strip based cuts are not applied.

In all three cases, for Mock data files, the truth information was removed from public access and stored in a separate undisclosed location.

Because of their good combination of portability, ease of use and flexibility, the MDC Standard ntuples produced with the Minossoft frozen release R1.12 were chosen to serve as the basic input for the analysis work presented in this thesis.

Further details related to MINOS standard reconstruction and the structure and usage of MINOS standard ntuples is available in Ref. [79] and Ref. [80]. 


\subsection{The NueAna framework}

The NueAna framework provides an infrastructure for development of $\nu_{\mu} \rightarrow \nu_{e}$ analyses. It allows easy integration and sharing of variables generated by each analysis, including tools to generate probability of identification (PID) trees, This sharing facilitates straightforward analysis comparisons and efficiency and sensitivity studies. The framework also includes one of the most extensively developed event displays available in the MINOS collaboration, dubbed the Ultimate Scanning Machine (USM); it is extremely useful for gauging analysis performance on an event by event basis, and it is the foundation of the ongoing hand scan analysis effort. The NueAna framework is an integral part of the main Minossoft CVS repository in the form of the NueAna package, being thus available and transparent to the collaboration. Different NueAna jobs can be selected at run time by using the loon executable in combination with different JobControl configuration macros. The type of job and run parameters are fully defined in the JobControl scripts, in analogous manner to reconstruction jobs.

The basic input to NueAna is the Standard ntuple format. The ntuples are read and processed to produce analysis ntuples. These contain most of the original reconstructed variables and new variables calculated either from the existing variables or from alternative reconstruction methods based on the Standard ntuples strip information. Most of the new variables result from integration of previously developed $\mathrm{C}++$ classes, used on independent analyses, as objects inheriting from a common class. The main branches of variables in the NueAna ntuples are the following:

i) AnalysisNtuples: Most of the original reconstruction variables, and also any newly calculated ones that are agreed to represent a common set of useful variables for every MINOS analysis group.

ii) MST (Minimal Spanning Tree): Obtained from applying a minimal spanning tree algorithm to perform shower reconstruction on event strip information.

iii) FracVar: Calculated by taking different fractions of the total number of planes in an event. 
iv) ShowerFit: Originating from fitting longitudinal shower profiles to the event strip information in $\mathrm{U}$ and $\mathrm{V}$ views.

v) HitCalc: Calculated from 3D hits obtained by matching of $\mathrm{U}$ and $\mathrm{V}$ strips.

vi) AngCluster: Variables computed from applying a clustering algorithm to the 3D hits referred to in the previous item.

vii) AngFitCluster: Result from fitting longitudinal and transverse shower profiles to the clusters calculated by the AngCluster object.

The last three items were developed by the author at Tufts and will be treated in further detail in the following sections. A complete variable glossary of the NueAna ntuple contents is presented in Appendix B.

At this stage, the NueAna ntuples can be fed to independent analyses, such as the Multivariate Discriminant Analysis (MDA), object of this thesis, or Artificial Neural Network (ANN) and Decision Tree (DT) analyses. After event classification is performed, the NueAna framework provides tools to transform each analysis' output in PID trees, which connect the classification to both the Standard and NueAna ntuples. In this way, easy comparison of results between different analyses is possible, and an input to the USM that allows comparison of each event's PID with the truth information (if available), while simultaneously visualizing the event and relevant plots of NueAna variables, is provided. The PID trees may then be used to create re-weighted trees, from which sensitivity and oscillation parameter fit plots can be calculated. The NueAna framework further provides macros to automatically generate Near-Far comparison plots for each variable inside the NueAna ntuple.

A schematic summary of the NueAna framework structuring is shown in Fig. 3.5.

\subsection{D Hit Shower Reconstruction}

As part of the event classification efforts undertaken by the $\nu_{\mu} \rightarrow \nu_{e}$ analysis group, Prof. J. Schneps and the author proposed a set of discriminating variables calculated 


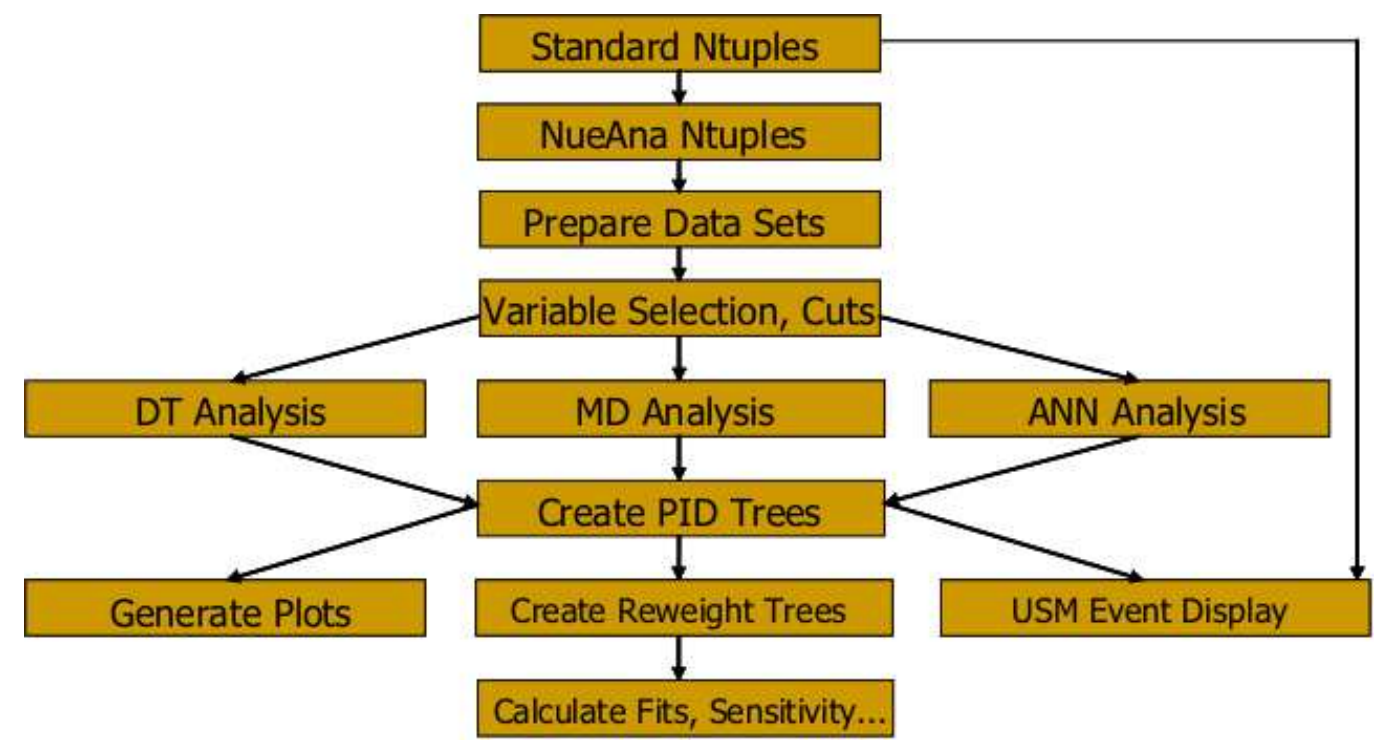

Figure 3.5: Organization of the analysis tools implemented by the NueAna framework. Starting from the Standard ntuples, NueAna ntuples are created and formatted to the input specifications of each type of analysis. The PID trees produced from each analysis output allow for generation of performance plots, individual variable plots or event scanning via the USM Event Display.

from 3D hits, which are determined from matching strips in consecutive detector planes. Additional variables are obtained by performing an alternative shower reconstruction based on clustering of the $3 \mathrm{D}$ hits via a nearest-neighbor method. This is followed by fitting the determined primary cluster with shower profiles. These fits allow the relevant properties of the shower to be characterized, such as transverse dispersion or maximum energy deposition, which are different for electromagnetic and hadronic showers. The next Sections describe in detail the 3D hit reconstruction, implemented as part of the NueAna framework, and the Tufts variables derived from them.

\subsubsection{D Hit Matching}

As noted in Chapter 2, the MINOS detector planes were erected in such a way that strip orientation is always alternated between $\mathrm{U}$ and $\mathrm{V}$ for every consecutive plane. The $\mathrm{U}$ and $\mathrm{V}$ strip orientations make an angle of $90^{\circ}$ with each other and an angle of 
$\pm 45^{\circ}$ with the horizontal. At this point it is useful to describe the MINOS coordinate systems [81]. The coordinate systems used by MINOS are right-handed. An $x$ $y-z$ system is used, where $+z$ corresponds to the horizontal component of the beam direction, $+y$ is locally vertical and $+x$ chosen such that the coordinate system is righthanded. Within the MINOS collaboration it is common to refer to $+x$ as detector west, $+y$ as detector up and $+z$ as detector north. The origin of the the detector coordinate system was chosen to be the center of plane 0 for both the Near and Far cases. The illustration displayed in Fig. 3.6 summarizes and further clarifies the coordinate systems used by MINOS.

The portion of MINOS event reconstruction concerned with track or shower finding and fitting is based on 2D algorithms that analyze separate UZ and VZ projections of each event. Depending on their spatial and timing parameters, the results for each view are then matched to form 3D tracks or showers. This procedure renders the computational implementation of the algorithm cumbersome, since two separate lists of strips have to be carried through the method, and also CPU intensive, as the reconstruction algorithm is always applied twice per event. In addition, since UZ or VZ event projections do not contain $\mathrm{V}$ planes or $\mathrm{U}$ planes, respectively, the effects of the granularity of the detector are enhanced and less information is fed to each pass of the reconstruction algorithm. This is a clear disadvantage in the case of the $\nu_{\mu} \rightarrow \nu_{e}$ analysis, where topological differentiation of electromagnetic and hadronic showers is paramount. Finally, 3D based visualizations of an event containing the complete strip information may generally convey more information to the human eye than the separate UZ, VZ representations.

The independent shower reconstruction done at Tufts is fully based on a preliminary matching of 3D hits at the strip level, and never makes use of the 2D UZ and VZ projections. The algorithm used for 3D hit matching within an event is rather simple and is described below:

1) The starting point is set at the plane where the standard MINOS reconstruction placed the event vertex (VertexPlane).

2) For every possible intersection between occupied perpendicular strips in Vertex- 


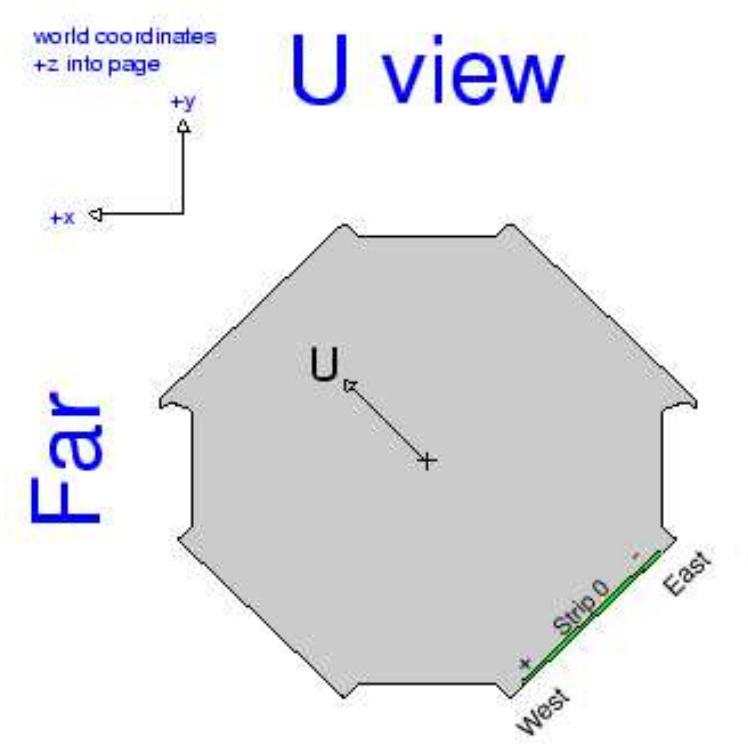

V view
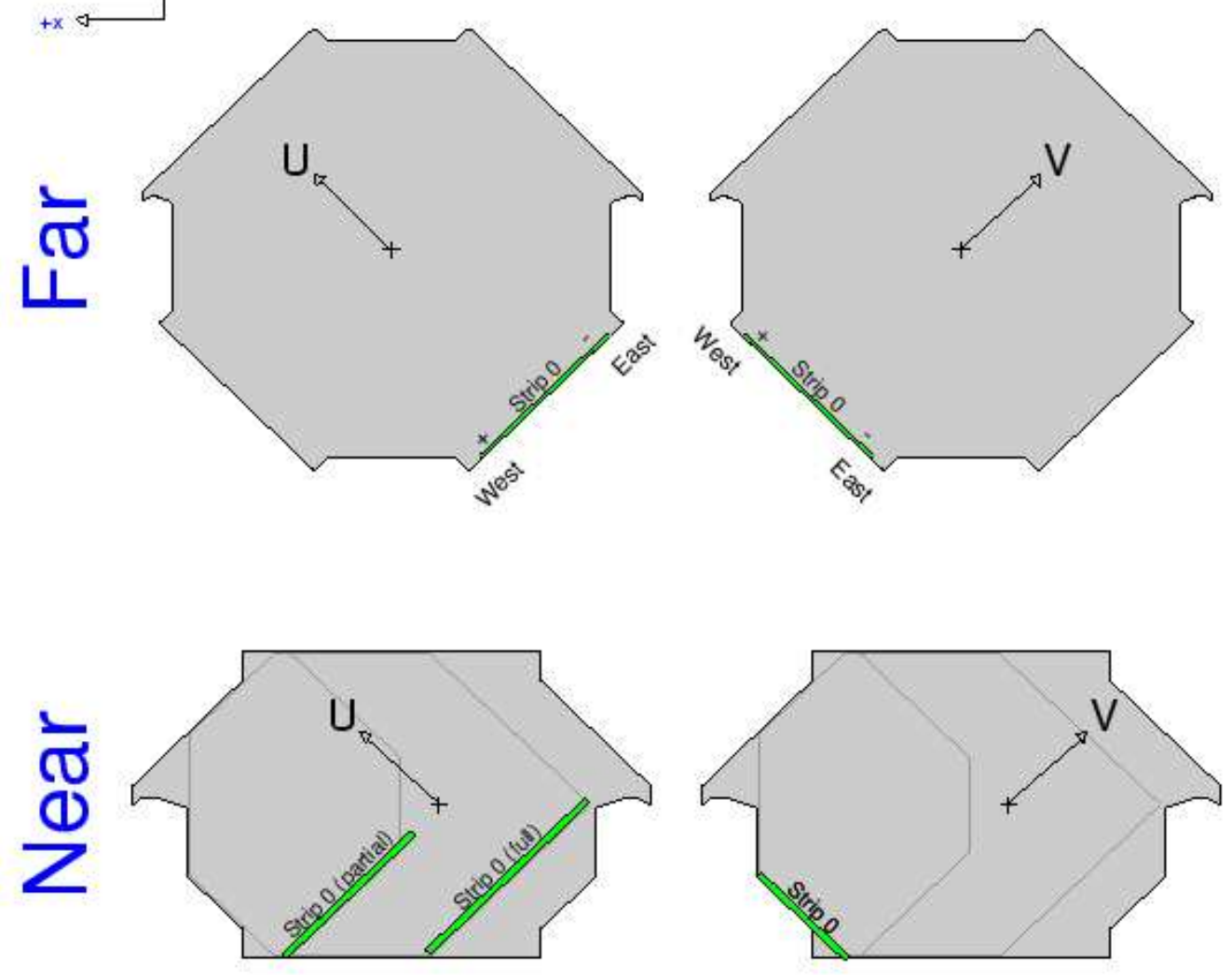

Figure 3.6: The coordinate systems used in the MINOS Far and Near detectors. The $u-v$ origin is located at the center of the coil. Plane orientations are referred to by the axis mapped out by the individual strips and not by the axis along which they lie. Strip numbers start with zero and increment with increasing $u$ or $v$. The strip 0 for each detector orientation is highlighted in green.

Plane and VertexPlane+1, the difference in strip energy is computed if the total energy contained in both strips is more than $4 \mathrm{PE}$.

3) If the energy difference between the occupied strips in consecutive planes is lower than a tunable threshold, a 3D hit is added to a list and its $x-y-z$ coordinates are calculated. Its energy is obtained from the semi-sum of the values of energy deposited in each of the two strips that define the hit. 
4) The starting plane number is incremented by 1 (corresponding to moving in the $+z$ direction) and steps 2 ) and 3) are repeated. The procedure stops when the last occupied plane in the event is reached.

The algorithm is summarized in Fig. 3.7.

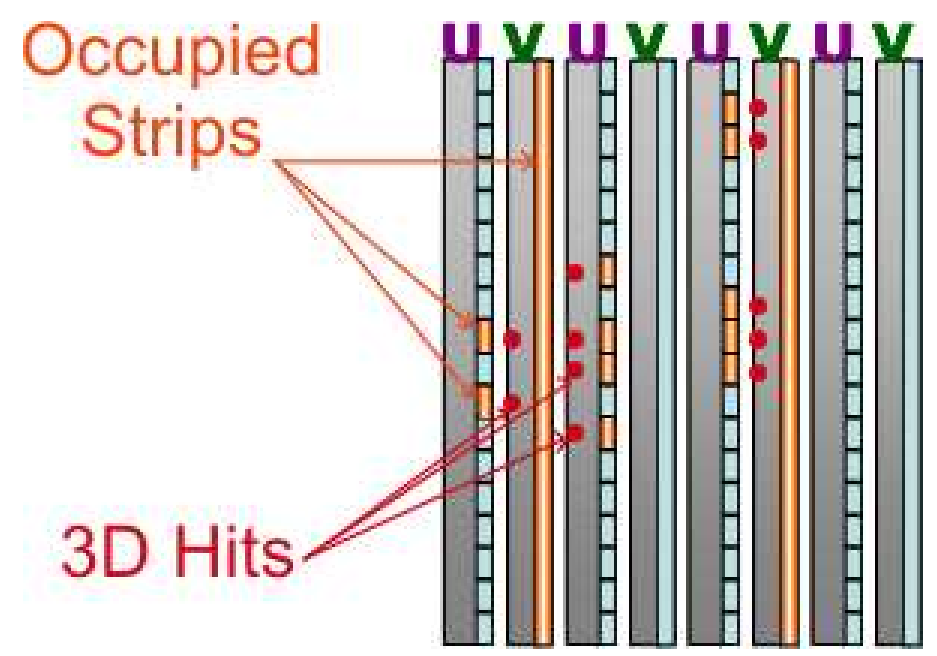

Figure 3.7: The 3D hit matching algorithm applied in a detector longitudinal section. Each pair of occupied strips (shown in orange) in consecutive planes originates a hit. The hit location is marked by a red circle. Each hit is assigned an energy equivalent to the semi-sum of the energy of its two corresponding strips.

Because a hit results from the intersection of two strips each with width $w=4.1 \mathrm{~cm}$, its transverse position resolution may be estimated to be $\sigma_{x y}=w^{3 / 2} / \sqrt{12}=2.40 \mathrm{~cm}$. The most problematic consequence of applying this algorithm in the MINOS case involves the potential for finding numerous unphysical hits, referred to as "ghost hits", derived from the position uncertainty along the full strip length for a particle traversing an occupied strip. A partial solution to this problem is provided by step (3) above, which corresponds to demanding that the energy loss by a potential particle from a plane to the next is consistent with a tunable parameter. For the analysis of this thesis, a value of $10 \mathrm{MEU} \approx 400 \mathrm{MeV}$, favoring quasi-elastic charged current $\nu_{e}$ interactions was chosen. Note that this threshold can eliminate physical hits in the first planes following the interaction vertex of a highly energetic particle $(\mathrm{E}>10 \mathrm{GeV})$, but as we will see later, this type of interaction will not be included in this analysis. 
Because the hit matching algorithm starts at the vertex obtained through Standard Reconstruction and proceeds in the direction of increasing plane number, any strip occupation due to back-scattering will not be present in the 3D hit representation of the event. Fig. 3.8 illustrates the results of the application of the algorithm to a MINOS event.
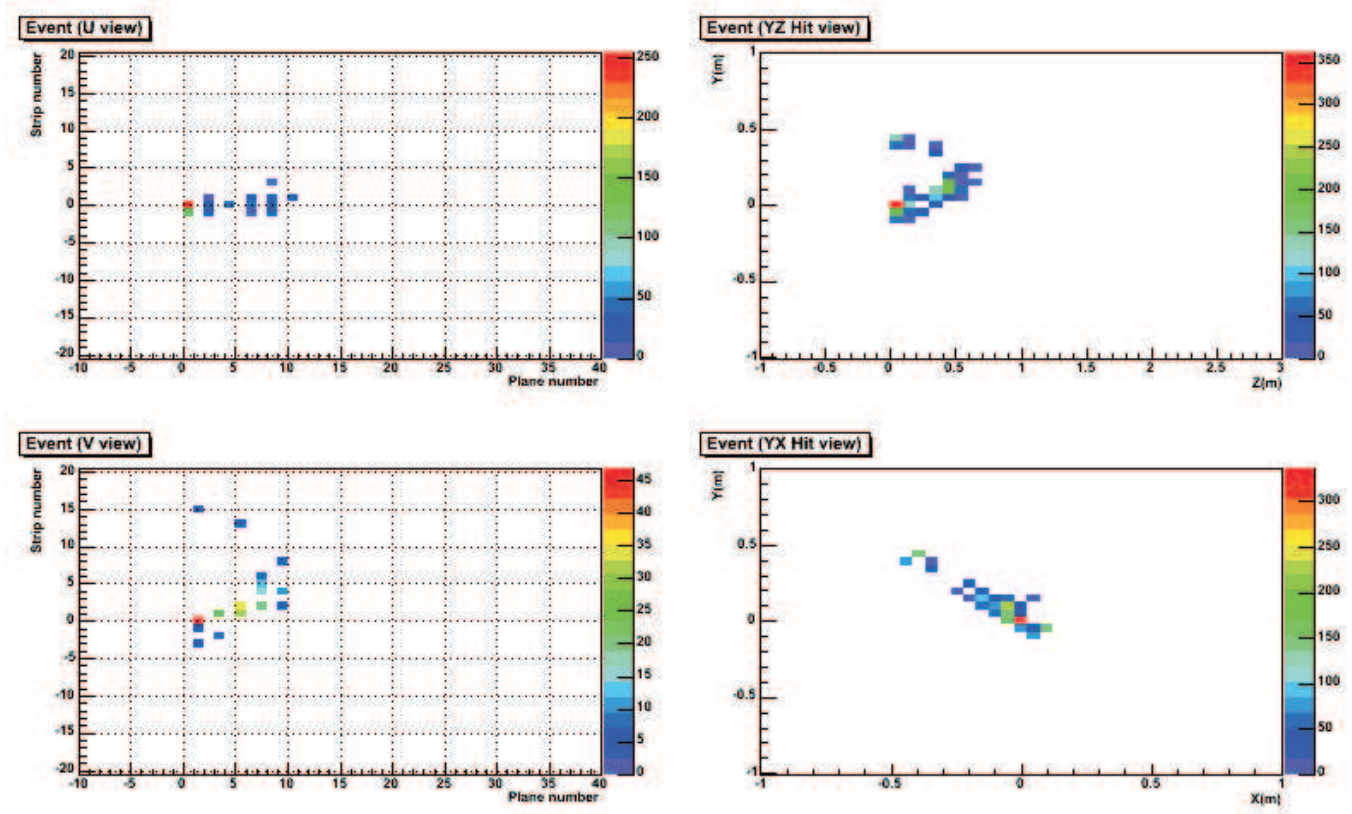

Figure 3.8: A charged current $\nu_{e}$ event shown in $\mathrm{U}$ vs plane number and $\mathrm{V}$ vs plane number representations (left plots) and in YZ and XY representations (right plots) after 3D hit matching.

\subsubsection{Angular Clustering}

The Standard Reconstruction available at the time release R1.12 was used to process the MDC sample contained a shower finding algorithm, but did not include any shower fitting tools. The standard reconstruction algorithm starts by looking for strip associations within planes, assembling together strips within 5 strips of each other that are also within a 1 ns time window. Then, cluster formation takes place in the 2D UZ and VZ projections by applying topological constraints to the strip associations, such as a minimal longitudinal span of 3 planes and a minimal number 
of 3 strips in the plane corresponding to the cluster's maximum transverse width. The $\mathrm{U}$ and $\mathrm{V}$ clusters thus formed are compared to each other and combined into 3D showers if the $\mathrm{Z}$ position of their first planes is within 6 planes and their beginning times are within 30 ns. No further reconstruction is applied to these showers.

At Tufts, a different approach to clustering of MINOS showering events was undertaken to attempt to maximize discrimination between hadronic and electromagnetic showers. Clustering is performed via a seedless nearest-neighbor algorithm applied to a representation of the event in spherical coordinates, calculated from transformation of the $x-y-z$ coordinates of the 3D hits, which are obtained as described in the previous section. The use of a spherical coordinate representation allows for simplification of clustering, which can still be done in $2 \mathrm{D}$, whilst fully conserving the directional information of the shower. One can produce several types of spherical coordinate representations of an event. For instance, taking the polar axis to be along each one of the cartesian axes will produce three different representations, as shown in Fig. 3.9. Since most of the events develop longitudinally along the beam direction, choosing the representation with polar angle along $z$ would not be useful, as the events will typically look like density regions distributed in a narrow band close to $\cos \theta_{z}=1$. The chosen representation is a combination of the representations obtained by taking the polar angle to be along the transverse coordinates, resulting in a $\cos \theta_{x}$ vs $\cos \theta_{y}$ projection of the $3 \mathrm{D}$ hits, also shown in Fig. 3.9. In this representation, a purely forward showering event would appear as a density region centered around the origin of the coordinates. After clustering in this view is completed, the hits composing the 3D showers along with shower direction are immediately available and cluster matching is not required. Because clustering is applied to such type of projection, based on polar angles calculated from the 3D hits, this method is informally known as "Angular Clustering".

The algorithm used to perform the clustering is described in detail below and is illustrated via graphical display in Fig. 3.10.

1) In the $\cos \theta_{x}$ vs $\cos \theta_{y}$ space, bound within a $[-1,1]$ interval in both coordinates, the distance of each hit to all other hits is calculated and indexed, omitting reciprocal 

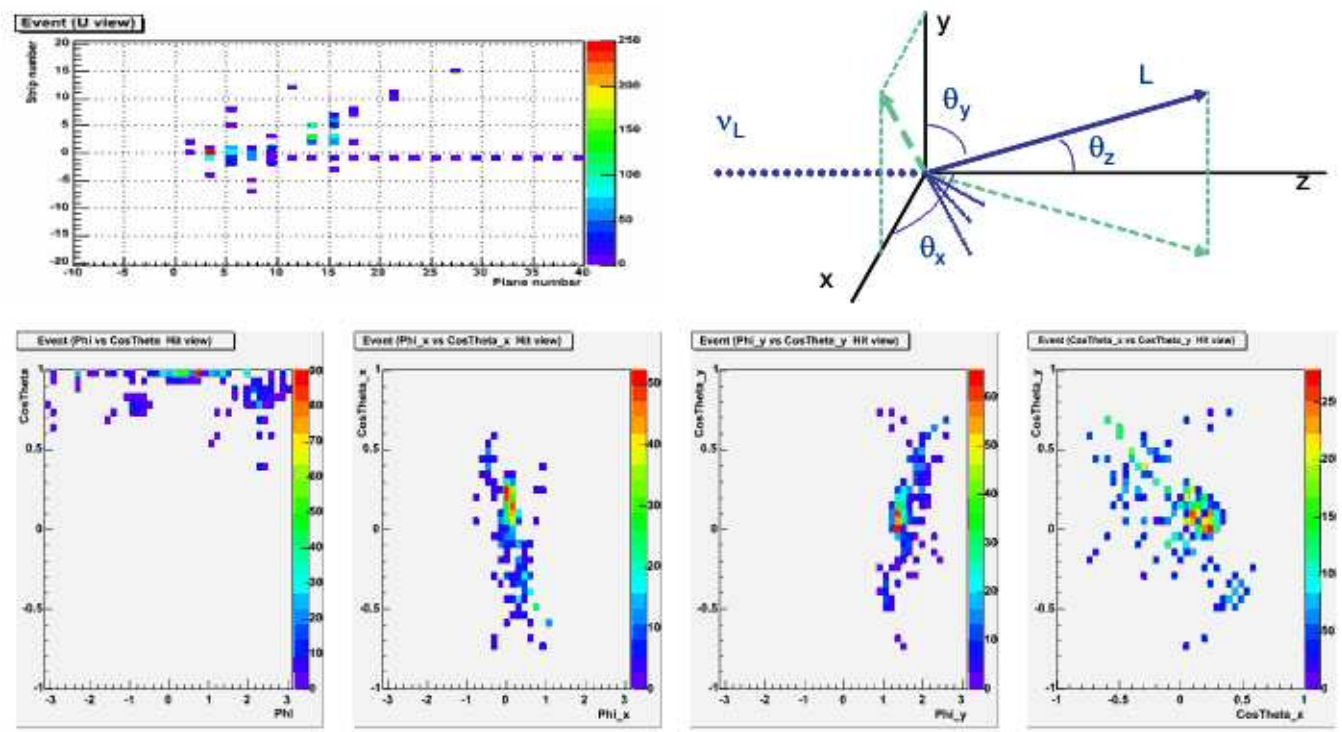

Figure 3.9: Examples of spherical coordinate representations of an event. The top part show the usual UZ event representation (left) and a diagram of a typical charged current event with the different polar angles made explicit (right). The bottom plots illustrate the different event representations obtained by choosing a polar angle along different axes. The bottom right corner plot corresponds to the event representation used in the angular clustering method.

distances in the process.

2) Following the nearest-neighbor concept, all of the hits within some tunable radius of a given hit are aggregated, with already used distances being discarded as the method progresses. Given that no preliminary seeding takes place and all hits are treated equally, the hits can be assigned multiple times to overlapping aggregates.

3) The degeneracies introduced in the previous step are resolved by histogramming the centroids of all aggregates and computing the bounds of each high density region via a recursive algorithm. Regions containing less than a small tunable number of centroids are discarded as noise. By introducing recursion at a higher level where hits have already been associated in high density regions, this method requires considerably less computing resources than a brute-force recursive algorithm that would act on individual hits. 
4) From the set of aggregates for which the centroid falls within the region bounds, the one containing the most hits is considered a cluster, thus lifting the degeneracy.

5) The cluster encompassing the largest number of hits is identified as the primary shower of the event.

6) The hits within the first 3 planes of the event vertex are added to the primary shower so as to improve the determination of shower direction. At this point, a list of clusters and all the hits contained in each of them is available for further processing.
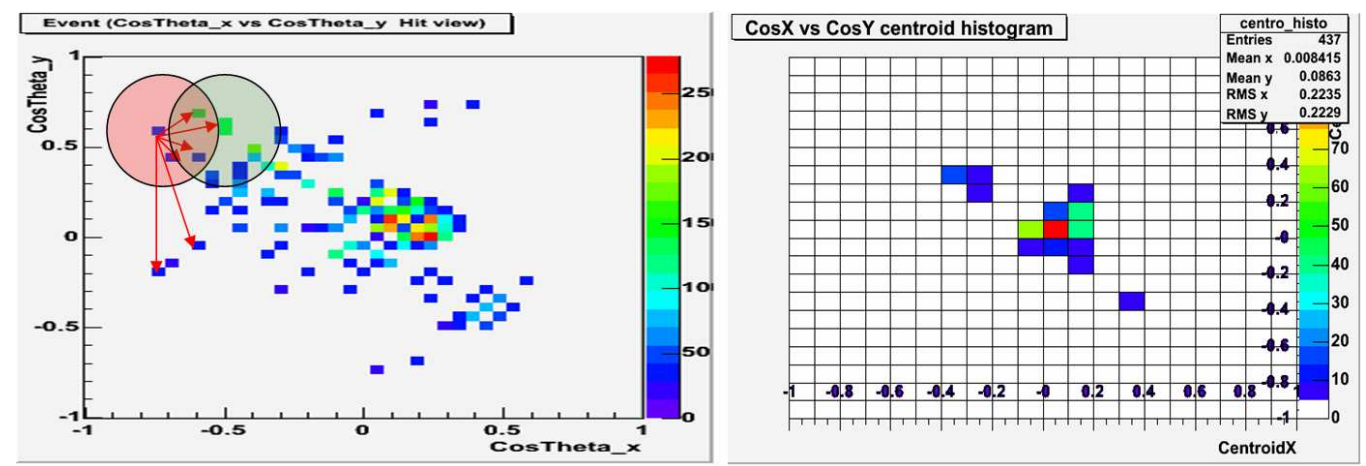

Figure 3.10: The illustration on the left summarizes the first steps of the angular clustering algorithm. For every hit, one calculates the distances to all other hits as exemplified by the red arrows. All the hits closer to a given hit than some radius form an aggregate, e.g. the hits within the red circle. The procedure is repeated for every hit, and degenerate hits belonging to two aggregates can occur, as is the case for the two hits in the overlapping region between the red and green circles. The centroids of all aggregates are histogrammed, as illustrated on the graph on the right, and a recursive algorithm employed to find the boundaries of each density region. A cluster is defined as the largest aggregate whose centroid falls within those bounds.

The use of ROOT's TVector3 and TRotation objects in the implementation of the angular clustering method decisively contributes to code simplification, as these objects offer the necessary methods to compute non-cartesian hit coordinates and perform any type of axis rotation. Fig. 3.11 illustrates the application of angular clustering to a showering event. 
As can be inferred from the description above, this method has two tunable parameters, namely the nearest-neighbor radius and the noise. In this analysis, the method's performance was optimized for quasi-elastic $\nu_{e} \mathrm{CC}$ interactions, which give rise to the most straightforward type of events that can be identified as a $\nu_{\mu} \rightarrow \nu_{e}$ signal. A value of 0.5 was used for the radius and a value of 3 for the noise parameter.

The calculation of the shower direction relies upon good performance of the standard reconstruction's vertex finder. Due to the intrinsic spatial resolution of the detector, combined with the small solid angle scanned by the clustering method in the first planes after the vertex, the hits located closer to the vertex, which carry a considerable amount of the total event energy, can easily be missed. In order to minimize this shortcoming, all the hits within 3 planes after the vertex are added to the primary shower.

The fitting procedure developed for the primary showers identified through angular clustering is discussed in the next section.

\subsubsection{Shower Fitting}

Using the 3D hit spatial coordinate centroid of the primary shower obtained through clustering, along with the vertex found by standard reconstruction, one can determine the average shower direction and quantify the shower characteristics by fitting shower profiles along that direction. This method improves on previous fitting techniques used in the NueAna analysis, which are limited to fitting the complete set of strips in an event along the $+z$ direction.

\section{Electromagnetic showers}

Electromagnetic cascade showers were first observed in high energy cosmic rays and were discovered by Blackett and Occhialini in 1933 [82]. The degradation of the energy of the incident particle results in a multiplicative process, regulated by the magnitude of the total incident energy. High energy electrons lose most of their energy through radiation via the bremsstrahlung effect, thus producing highly energetic photons. The photons undergo materialization, as pair production processes are dominant for energies above $100 \mathrm{MeV}$, or produce Compton electrons. The resulting electrons and 
positrons radiate more photons which continue the cascading process while they are generated with sufficiently large energy. For the energy ranges relevant to MINOS, the bremsstrahlung and pair production processes are well approximated by the BetheHeitler formula [83]. The characteristic amount of matter traversed for these related interactions is called the radiation length, which will be identified by $X_{g 0}$, if measured in $\mathrm{gcm}^{-2}$, or $X_{0}=\frac{X_{g 0}}{\rho}$, if measured in $\mathrm{cm}$, where $\rho$ is the material density in $\mathrm{gcm}^{-3}$. The radiation length represents both the average distance over which a high energy electron loses all but $1 / e$ of its energy by bremsstrahlung, and $7 / 9$ of the conversion length, defined as the mean free path for pair production by a high energy photon. As a consequence, the radiation length is a natural scale length for describing high energy electromagnetic cascades in a material. It is given by [84]

$$
\frac{1}{X_{g 0}}=4 \alpha \frac{N_{A}}{A} Z(Z+\zeta) r_{e}^{2} \ln \frac{183}{Z^{1 / 3}} \quad\left[\mathrm{~cm}^{2} \mathrm{~g}^{-1}\right],
$$

where $\alpha$ is the fine structure constant, $N_{A}$ is the Avogadro constant, $Z$ the atomic number of the medium, $A$ is the atomic weight of the medium, and $r_{e}$ is the classical electron radius. $\zeta$ is the correction due to the contribution of atomic electrons to the bremsstrahlung process and has values between 1.2 and 1.4. For quick estimates, an approximation to Eq. 3.1 is often used:

$$
X_{g 0} \approx 180 \frac{A}{Z^{2}} \quad\left[\mathrm{gcm}^{-2}\right] .
$$

When more than one absorber is present in the showering medium, the overall radiation length is expressed as

$$
\frac{1}{X_{g 0}}=\sum_{i} \frac{f_{i}}{X_{g 0 i}}
$$

with $f_{i}$ and $X_{g 0 i}$ the fraction by weight and the radiation length of the absorber $i$. The corresponding density is computed from

$$
\frac{1}{\rho}=\sum_{i} \frac{f_{i}}{\rho_{i}}
$$

where $\rho_{i}$ is the density of the absorber $i$.

Some relevant radiation length values for different materials are shown in Table 3.2 . 
The depth at which the largest number of secondary particles created during the multiplication process is reached, and correspondingly, where the largest energy dissipation occurs, is referred to as $t_{\max }$. Beyond this depth, the cascade decays slowly. As the cascade evolves along the depth of a calorimeter such as the MINOS Far detector, more and more electrons fall into an energy range where collision energy losses dominate radiation energy losses, effectively damping the multiplication process. The multiplication process due to a single electron will practically stop when the electron reaches a critical energy $\epsilon_{c}$, the energy at which an electron loses as much energy in collisions as in radiation, expressed as

$$
\epsilon_{c}=B\left(\frac{Z X_{g 0}}{A}\right)^{h} \quad[\mathrm{MeV}],
$$

with $B=2.66$ and $h=1.11$.

\section{Longitudinal Shower Fitting}

Theoretical descriptions of cascade showers originate from papers by Bhabha and Heitler [85] and Carlson and Oppenheimer [86]. However, the complexity of interaction mechanisms with the calorimeter medium, in particular in the case of low energy components of the cascade, requires treatments where part of the physical processes is neglected or approximated. The use of shower simulation codes which reproduce the behavior of electromagnetic showers in various media to high accuracy, allows for the inclusion, in a phenomenological way, of the various effects that the analytical approach fails to describe.

The longitudinal shower distribution used for fitting in the AngCluster module results from one of these phenomenological approaches. It is valid up to energies of $100 \mathrm{GeV}$ and it is described by [87]

$$
\frac{d E}{d t}=E_{0} \frac{b^{a+1}}{\Gamma(a+1)} t^{a} e^{-b t},
$$

where $t$ measures depth of the cascade in units of $X_{0}, E_{0}$ is the energy of the incoming particle in $\mathrm{GeV}, \Gamma$ is the Euler function and $a$ and $b$ are parameters to be determined, describing the shower rise and shower decay, respectively. In this formulation, the 
position of the shower maximum is located at

$$
t_{\max }=\frac{a}{b}=\ln \left(\frac{E_{0}}{\epsilon_{c}}\right)+d \quad\left[X_{0}\right]
$$

and $d=-0.5$ or 0.5 for incident electrons or photons, respectively.

The fitting is performed over the 3D hits belonging to the primary shower determined by angular clustering. The hit reference frame is rotated so that $+z$ is along the shower direction and the coordinates are transformed to units of $X_{0}$. Fig. 3.11 illustrates the longitudinal energy distribution of a typical electromagnetic shower and the shape of the profiles used to fit the distribution.

\section{Transverse Shower Fitting}

During the shower development, the energy is degraded into low energy electrons through ionization, Compton scattering and photoelectric interactions, which in turn generate electrons that dissipate their energy predominantly by collision. The transverse spread of the cascade is caused by diverse underlying physics processes. The photoelectric interactions and Compton scattering generate secondary electrons that are not aligned with the incoming photon direction. In addition, the secondary Compton photons are not along the primary photon directions, contributing to the widening of the cascade. Finally, multiple Coulomb scatterings of the electrons below the radiation threshold, but with enough energy to travel away from the shower core, lead to the spread of electron directions out of the axis defined by the primary particle direction. The transverse depth unit of a shower is called the Molière radius and is defined as

$$
R_{M}=\left(\frac{E_{M}}{\epsilon_{c}}\right) X_{0}
$$

where $E_{M}=\sqrt{\frac{4 \pi}{\alpha}}\left(m_{e} c^{2}\right)=21.2 \mathrm{MeV}$ and $\epsilon_{c}$ is the critical energy defined in Eq. 3.5. A quick estimate of the Molière radius of a material can be obtained from

$$
R_{g M} \approx 7 \frac{A}{Z} \quad\left[\mathrm{gcm}^{-2}\right]
$$

For a material composed of several absorbers, the total Molière radius is estimated as

$$
\frac{1}{R_{g M}}=\frac{1}{E_{M}} \sum_{i}\left(f_{i} \frac{\epsilon_{c, i}}{X_{g 0 i}}\right)
$$


where $f_{i}, \epsilon_{c, i}$ and $X_{g 0 i}$ are the weight fraction, critical energy and radiation length of absorber $i$, respectively. Table 3.2 shows relevant tabulated and calculated values for the Molière radius of different materials. From the tabulated values and the knowledge that a MINOS plane has a mass of 11.5 ton, a maximum width of $w=8 \mathrm{~m}$ and an area of $w^{2}-2\left(\frac{w}{2+\sqrt{2}}\right)$, it can be shown that a single MINOS plane corresponds to 1.54 radiation lengths and a single detector strip corresponds to 1.05 Molière radii.

\begin{tabular}{|c|c|c|c|c|c|c|}
\hline Material & $\rho\left\{\mathrm{gcm}^{-3}\right\}$ & $f_{i}$ & $X_{g 0}\left\{\mathrm{gcm}^{-2}\right\}$ & $X_{0}\{\mathrm{~cm}\}$ & $\epsilon_{c}\{\mathrm{MeV}\}$ & $R_{m}\{\mathrm{~cm}\}$ \\
\hline \hline $\mathrm{Fe}$ & 7.87 & 0.939 & 13.84 & 1.76 & 21.03 & 1.77 \\
\hline Al & 2.70 & 0.0127 & 24.01 & 8.90 & 40.25 & 4.68 \\
\hline Polystyrene & 1.03 & 0.0480 & 43.72 & 42.40 & 88.50 & 10.14 \\
\hline Air(STP) & $1.24 \times 10^{-3}$ & $1.42 \times 10^{-4}$ & 36.66 & $3.04 \times 10^{4}$ & 67.80 & 11.46 \\
\hline 1 MINOS plane & 3.50 & 1.00 & 14.38 & 4.10 & 22.23 & 3.91 \\
\hline
\end{tabular}

Table 3.2: Values of radiation length and Molière radius for the materials composing a MINOS detector plane, tabulated along with the fraction by weight for each material in a single detector plane. The calculated values for a single MINOS steel/scintillator plane are also shown.

Many parametrizations of the transverse shape of an electromagnetic shower have been introduced as a function of the calorimeter depth. A double exponential form is often employed, whereby the central core and peripheral halo of the shower are described. The parametrization used here was developed from a phenomenological analysis of data obtained from a silicon calorimeter composed of tungsten and uranium absorber layers, exposed to incoming electrons with energies of 2,4 and $6 \mathrm{GeV}$, undertaken by the SICAPO collaboration [88]. The radial energy profile is given by

$$
\frac{d E}{d r}=\frac{1}{N}\left[\exp \left(-\sqrt{\frac{r}{\lambda_{1}}}\right)+C_{12} \exp \left(-\frac{r}{\lambda_{2}}\right)\right],
$$

where $\lambda_{1}^{2}, \lambda_{2}$ are attenuation lengths in units of $R_{M}, C_{12}$ is the relative weight of the two exponential functions and $r$ represents the radial distance from the shower axis in units of $R_{M}$. $N$ is a normalization factor such that

$$
\int_{0}^{2 \pi} d \phi \int_{0}^{+\infty} \frac{d E}{d r} r d r=1
$$

with $\phi$ being the polar angle. The four parameters $\lambda_{1}, \lambda_{2}, C_{12}$ and $N$ are fitted to 
the 3D hits composing the primary shower, with the reference frame rotated so that $+z$ coincides with the shower axis, with coordinates in units of $R_{M}$.

Fig. 3.11 depicts an example of a transverse shower profile and the shape of the fitting function.
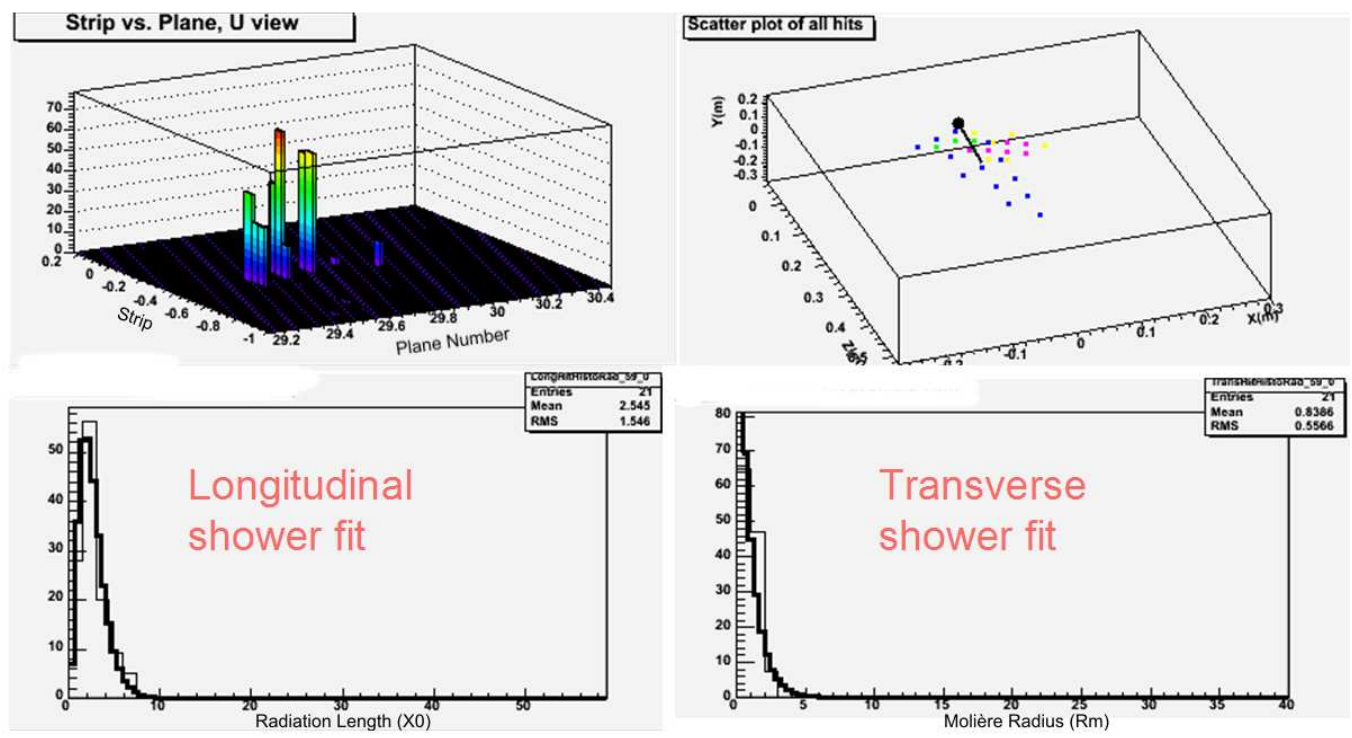

Figure 3.11: Angular clustering and fitting of a charged current quasi-elastic $\nu_{e}$ event. The top left plot depicts the strip representation of the event. On the top right, the same event is shown in a $3 \mathrm{D}$ hit representation after angular clustering. The different clusters found are presented in different colors. The black dot marks the vertex position and the black line follows the shower direction. The primary shower of the event is the largest cluster, composed of the blue hits. In the bottom left, the longitudinal energy distribution of the primary shower is shown with the fitted profile superimposed. The bottom right graph shows the transverse energy distribution for the primary shower and its corresponding fitted profile.

\subsection{The Tufts Analysis Variables}

Many useful discriminating variables can be extracted from the application of the methods described in this Section. From the 3D hit matching code, one can consider the angle made by the line defined by the vertex and the farthest hit with the beam direction. The distribution for charged current quasi-elastic events should be strongly biased towards forward events, thus small angles, whereas neutral current 
events should display a more isotropic distribution. One can also consider the projection of the hits in the $x y$ plane and define a separating line as the perpendicular to the line that goes from the vertex through the centroid of the event. The ratio between the number of hits on each side of the line gives a measure of the momentum asymmetry in the event. Neutral current events are expected to exhibit a higher degree of asymmetry and so have a variable distribution peaked at a value different from unity, a behavior opposite to the one expected for charged current events. The angular clustering method allows a primary shower in the event to be isolated and its direction calculated. The root-mean-square (RMS) distribution of the radial distance of the primary shower hits to the shower axis provides discrimination between electromagnetic and hadronic showers, as the hadronic shower distributions tend to display longer tails and less compact profiles. More precise differentiation between hadronic and electromagnetic showers can be obtained using the shower fitting parameters as discriminating variables.

Fig. 3.12 shows distributions of the variables referred to in the text for the main classes of events used in the analysis discussed in the next Chapter. All of the Tufts variables calculated in the NueAna framework are summarily described in Appendix B. 

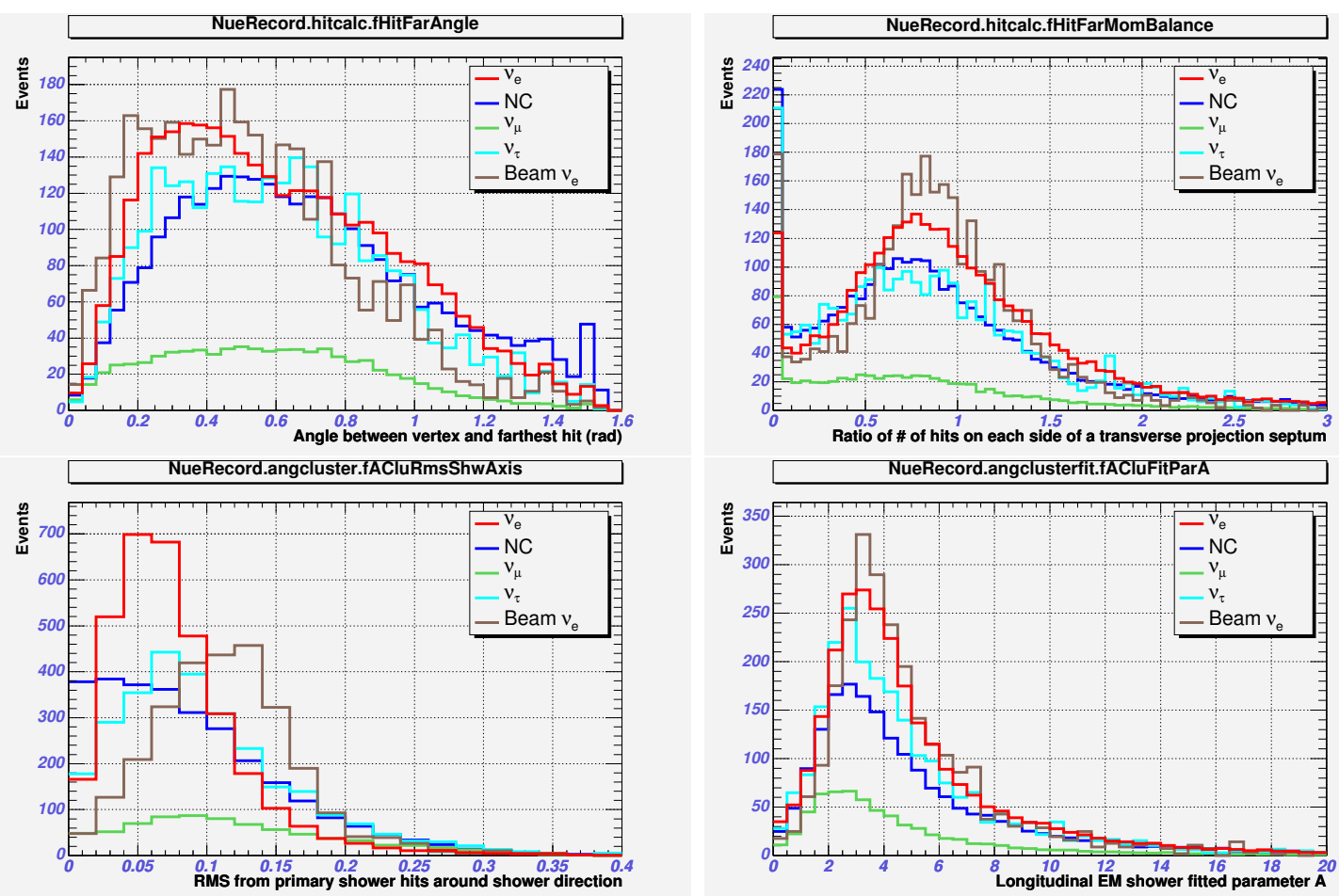

Figure 3.12: Distributions of some of the Tufts variables alluded to in the text. In order to better clarify the separation between the different types of events, the distributions for each class are scaled to the histogram with the largest integral value. 


\section{Chapter 4}

\section{The $\nu_{\mu} \rightarrow \nu_{e}$ Appearance Oscillation Analysis}

The MINOS experiment should be able to perform the most precise measurement of $\nu_{\mu} \rightarrow \nu_{e}$ appearance before the onset of the next generation of neutrino experiments, such as $\mathrm{NO} \nu \mathrm{A}$ and $\mathrm{T} 2 \mathrm{~K}$. This constitutes a very challenging measurement as it requires separation of a $\nu_{e}$ appearance signal severely limited by statistics, while making use of a sampling detector not optimized for showering event reconstruction.

The analysis described in this chapter was developed in collaboration with the Harvard MINOS group and, as other institutions joined the effort, eventually spawned the NueAna framework. Besides a visual scanning effort underway at Tufts University, $\nu_{e}$ event classification has been attempted through different multivariate methods, namely Decision Trees (DT) at Harvard, Artificial Neural Networks (ANN) at Stanford and Multivariate Discriminant Analysis at Tufts (MDA).

The first section of this chapter focuses on the characteristics of the MDA method. A detailed discussion of the different types of background involved, samples used, cuts applied and variable selection follows. The different MDA-based classification methods are described and the results obtained by application to the Far Monte Carlo sample are presented. 


\subsection{Multivariate Discriminant Analysis}

The analysis of experimental data almost always involves observations associated with various facets of the signal, particular backgrounds or systematic aspects of the experiment itself. In a general sense, data are always multivariate in nature.

One can define multivariate problems as those that are concerned with the analysis of $n$ points in $p$-space, i.e. each one of the experimental observations, detector interactions or events in our case, is associated with a $p$-dimensional vector of responses or variables. The goal of a multivariate analysis is to assign a set of these events, described by $p$-vectors of variables, to one of two or more groups.

\subsubsection{Principal Component Analysis}

A simple example of a multivariate data reduction technique is given by the Principal Component Analysis (PCA), developed by Karl Pearson in 1901 [89]. The basic idea in PCA is to describe the dispersion of an array of $n$ points in $p$-dimensional space by introducing a new set of orthogonal linear coordinates so that the sample variances (defined as the distance from the mean of the sample) of the given points with respect to these derived coordinates are in decreasing order of magnitude. Therefore, the first principal component is such that the projections of the given points onto it have maximum variance among all possible linear coordinates; the second principal component has maximum variance subject to being orthogonal to the first, and so on. The coordinates of the observations with respect to each of the principal component axes give the values for the new variables. These values are usually called principal component scores. Each of the new variables is a linear combination of the original $p$ variables, and is uncorrelated with the remaining new variables. The PCA technique is illustrated in Fig 4.1.

\subsubsection{The Fisher Discriminant Function}

The Principal Component Analysis represents the data along the directions of maximum variance. However, for some data distributions, this can severely counteract classification efforts, as shown in Fig 4.2. This classification problem was addressed 

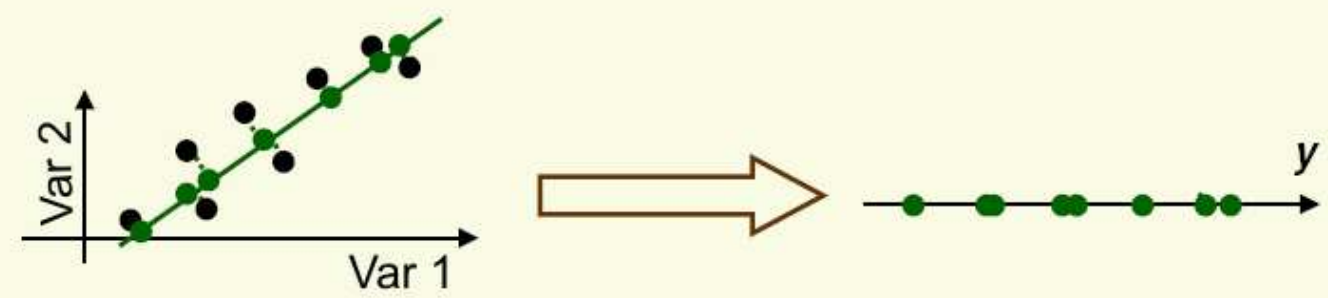

Figure 4.1: Illustration of the application of PCA to a 2D data set (black dots). The green line corresponds to the linear combination of var1 and var2 that minimizes the errors on the projections of the data points (green dots), thus lying along the direction that simultaneously maximizes the variance of every data point. This line defines the principal component axis and a $1 \mathrm{D}$ representation of the data through the variable $y$.
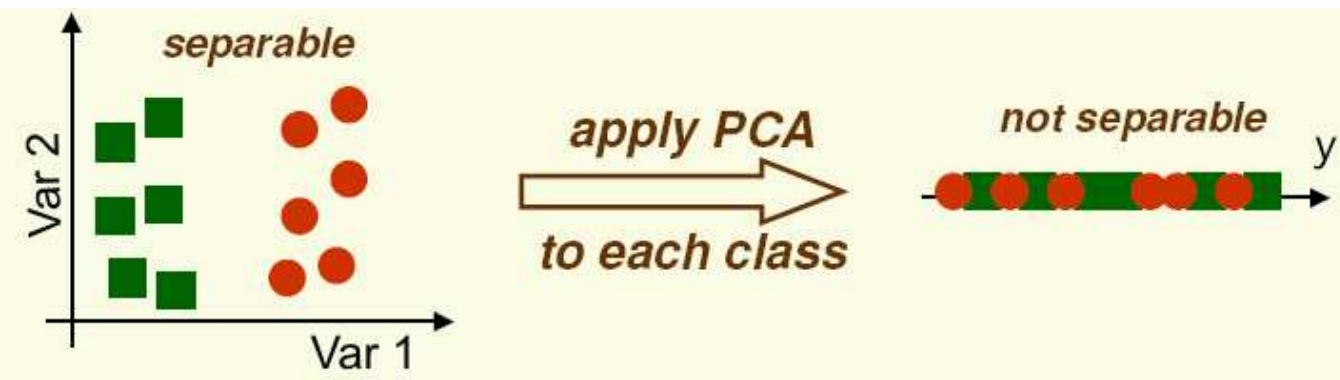

Figure 4.2: An example of a data set composed of observations divided in two classes. The principal component axis for these data is a vertical line approximately equidistant from the green and red classes of observations. The use of PCA for classification in this case is completely ineffective.

by Ronald Fisher in his 1936 paper [90], which introduces the Fisher Linear Discriminant (FLD). It is instructive to look at the application to the case of two group separation.

Consider two groups, $G_{1}$ and $G_{2}$, and their corresponding reference sets of observations (also referred to as training samples) in matrix representation, $\mathbf{X}_{1}$ and $\mathbf{X}_{2}$, such that the $n_{1}$ columns of $\mathbf{X}_{1}$ are $p$-dimensional observations on $n_{1}$ units known to come from $G_{1}$ and similarly, the $n_{2}$ columns of $\mathbf{X}_{2}$ are observations on $n_{2}$ units from $G_{2}$. One can thus calculate the vectors of means

$$
\overline{\mathbf{x}}_{m}=\frac{1}{n_{m}} \sum_{j=1}^{n_{m}} \mathbf{x}_{m j}
$$



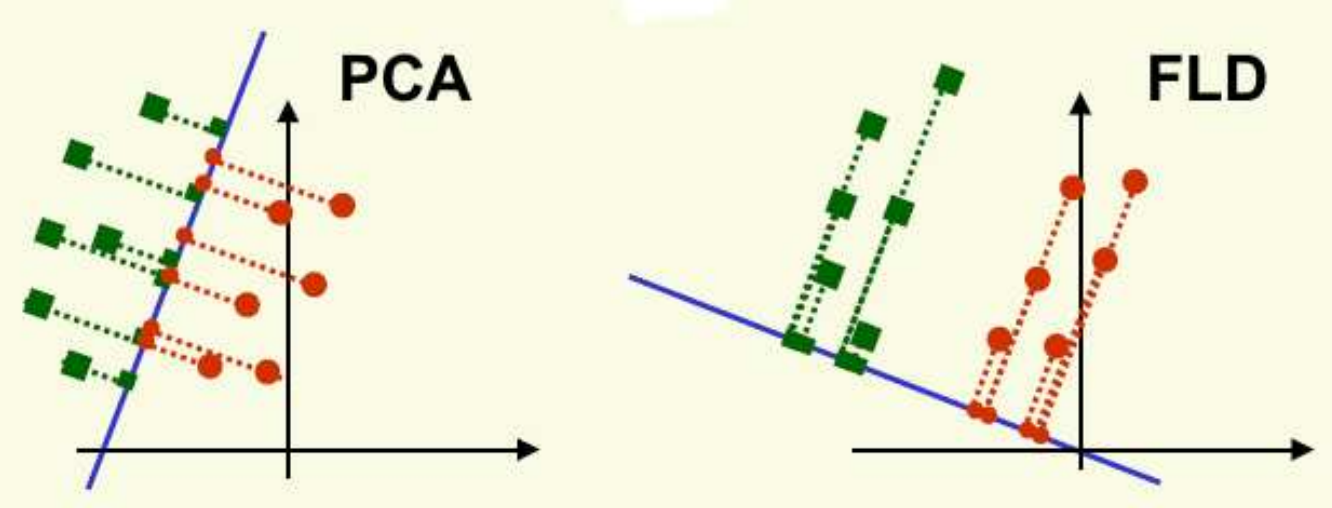

Figure 4.3: Illustration of the improvement in classification by applying the FLD technique (right) to a data set not separable through the PCA method (left). The Fisher Linear Discriminant (blue line in the plot on the right) lies along the direction that maximizes the separation between the means of the projections of the observations from the two classes or groups.

and the covariance matrices

$$
\mathbf{S}_{m}=\frac{1}{n_{m}-1} \sum_{j=1}^{n_{m}}\left(\mathbf{x}_{m j}-\overline{\mathbf{x}}_{m}\right)\left(\mathbf{x}_{m j}-\overline{\mathbf{x}}_{m}\right)^{t}
$$

where $m \in\{1,2\}$ and the superscript $t$ denotes transposing. The Fisher discriminant function is the linear combination of the $p$ original responses which exhibits the largest ratio of variance between the two groups relative to that within the groups, as shown in Fig. 4.3. . More explicitly, if the linear combination of the original $p$ variables is expressed as

$$
y=\sum_{i=1}^{p} a_{i} x_{i}=\mathbf{a}^{t} \mathbf{x}
$$

a two sample $t$-statistic for the variable $y$ may be written as

$$
T_{\mathbf{a}}=\frac{\mathbf{a}^{t}\left(\overline{\mathbf{x}}_{1}-\overline{\mathbf{x}}_{2}\right)}{\left[\mathbf{a}^{t} \mathbf{S a}\left(1 / n_{1}+1 / n_{2}\right)\right]^{1 / 2}}=\frac{1}{\left(1 / n_{1}+1 / n_{2}\right)^{1 / 2}} \frac{\left(\overline{\mathbf{y}}_{1}-\overline{\mathbf{y}}_{2}\right)}{\sigma_{y}},
$$

where $\sigma_{y}$ is the standard deviation in $y$ and the covariance matrix $\mathbf{S}$ is given by

$$
\mathbf{S}=\frac{\left(n_{1}-1\right) \mathbf{S}_{1}+\left(n_{2}-1\right) \mathbf{S}_{2}}{n_{1}+n_{2}-2}
$$

which accounts for the possibility of each of the two groups having differing covariance matrices. This $\mathbf{S}$ matrix is a particular case of the pooled within-groups covariance 
matrix, discussed below. The Fisher discriminant function is now obtained by choosing a so as to maximize $\left|T_{a}\right|$ or equivalently

$$
T_{\mathbf{a}}^{2}=\left(\frac{n_{1} n_{2}}{n_{1}+n_{2}}\right)\left[\frac{\mathbf{a}^{t}\left(\overline{\mathbf{x}}_{1}-\overline{\mathbf{x}}_{2}\right)\left(\overline{\mathbf{x}}_{1}-\overline{\mathbf{x}}_{2}\right)^{t} \mathbf{a}}{\mathbf{a}^{t} \mathbf{S a}}\right]=\left(\frac{n_{1} n_{2}}{n_{1}+n_{2}}\right)\left(\frac{\overline{\mathbf{y}}_{1}-\overline{\mathbf{y}}_{2}}{\sigma_{y}}\right)^{2} .
$$

The maximizing solution for the coefficients a is unique (up to a multiplicative constant) and is given by [91]

$$
\mathbf{a} \propto \mathbf{S}^{-1}\left(\overline{\mathbf{x}}_{1}-\overline{\mathbf{x}}_{2}\right)
$$

In the $p$-dimensional vector of observations, the vector a defines the direction of maximal group separation in the sense that the means of the projections of the observations from the two groups are maximally apart relative to the variance of the projections around their respective means. Furthermore, the variable $y$ can be seen as a rotation of the axis in the space of variables, such that the projection of events along the $y$ axis gives the clearest separation of the groups. $y$ is referred to as a linear discriminant variable and is also known as the first canonical variable. A graphical example of the FLD method application is shown in Fig. 4.4.

The generalization of the Fisher Linear Discriminant approach to more than two groups is reasonably straightforward and well documented in the literature ( $c f$. Ref. [91], Ref. [92] or Ref. [93]). Suppose we have $g$ groups $G_{1}, \ldots, G_{g}$, with the reference set of observations consisting of $n_{m}$ p-dimensional observations from $G_{m}$. The $m^{\text {th }}$ group will have a sample mean vector $\overline{\mathbf{x}}_{m}$ and sample covariance matrix $\mathbf{S}_{m}$. For the total set of $n=\sum_{m=1}^{g} n_{m}$ observations, one can compute an overall mean vector $\overline{\mathbf{x}}=\sum_{m=1}^{g} n_{m} \overline{\mathbf{x}}_{m} / n$ and a pooled within-groups covariance matrix

$$
\mathbf{W}=\frac{1}{n-1} \sum_{m=1}^{g}\left(n_{m}-1\right) \mathbf{S}_{m}
$$

We can also define the pooled between-groups covariance matrix

$$
\mathbf{B}=\frac{1}{g-1} \sum_{m=1}^{g} n_{m}\left(\overline{\mathbf{x}}_{m}-\overline{\mathbf{x}}\right)\left(\overline{\mathbf{x}}_{m}-\overline{\mathbf{x}}\right)^{t}
$$

which provides a summary of the dispersion among the group means in $p$-space. As before, if $y=\mathbf{a}^{t} \mathbf{x}$ denotes a linear combination of the original variables, the Fisher 

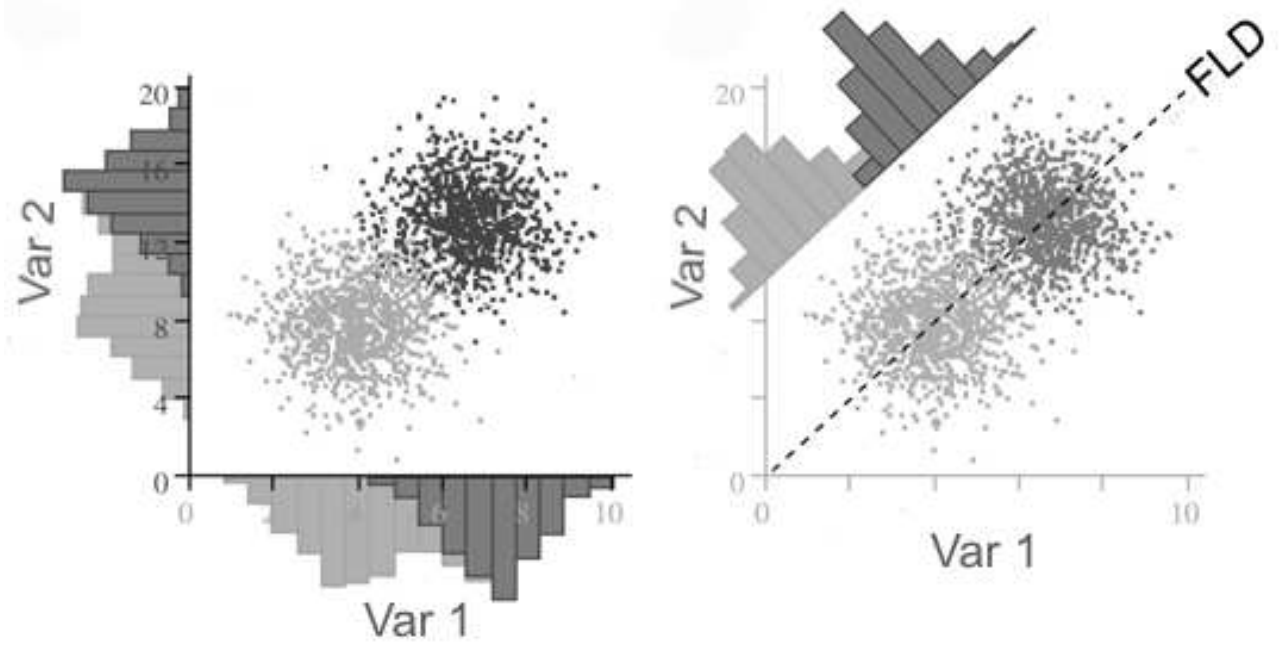

Figure 4.4: Illustration of the FLD ability to perform observation classification. In the left graph, using either one of two variables that characterize the data results in strong overlaps between the observations in the two groups. The FLD obtained (dashed line in the graph on the right) considerably increases the ability to separate the data into the two groups.

discriminant function can then be expressed as:

$$
F_{\mathbf{a}}=\frac{\mathbf{a}^{t} \mathbf{B a}}{\mathbf{a}^{t} \mathbf{W a}}
$$

For this function, the maximizing $\mathbf{a}$ is the eigenvector $\mathbf{a}_{1}$, corresponding to the largest eigenvalue, $c_{1}$, of $\mathbf{W}^{-1} \mathbf{B}$. The maximum value of the function is simply $F_{\mathbf{a}_{1}}=\mathbf{a}_{1}^{t} \mathbf{B} \mathbf{a}_{1} / \mathbf{a}_{1}^{t} \mathbf{W} \mathbf{a}_{1}=c_{1}$. The second largest eigenvalue $c_{2}$ will correspond to a second linear combination of variables, the eigenvector $\mathbf{a}_{2}$, and so on. Therefore, we can have a maximum of $g-1$ independent linear discriminant functions.

The multivariate discriminant method used in the analysis of this thesis is a generalization of the Fisher Linear Discriminant approach to 4 groups, one corresponding to signal and the other three to background, and also uses a somewhat more sophisticated discriminant function, to be described below. 


\subsubsection{Mahalanobis Distance}

The following discussion draws from the exposition in Ref. [94]. In the context of the two group example, consider the function

$$
T^{2}=\frac{\mathbf{a}^{t}\left(\overline{\mathbf{x}}_{1}-\overline{\mathbf{x}}_{2}\right)\left(\overline{\mathbf{x}}_{1}-\overline{\mathbf{x}}_{2}\right)^{t} \mathbf{a}}{\mathbf{a}^{t} \mathbf{S a}} .
$$

Inserting the maximizing solution found above, $\mathbf{a}=\mathbf{S}^{-1}\left(\overline{\mathbf{x}}_{1}-\overline{\mathbf{x}}_{2}\right)$, we have

$$
\begin{aligned}
T_{\text {max }}^{2} & =\frac{\left[\left(\overline{\mathbf{x}}_{1}-\overline{\mathbf{x}}_{2}\right)^{t} \mathbf{S}^{-1}\left(\overline{\mathbf{x}}_{1}-\overline{\mathbf{x}}_{2}\right)\right]^{2}}{\left(\overline{\mathbf{x}}_{1}-\overline{\mathbf{x}}_{2}\right)^{t} \mathbf{S}^{-1} \mathbf{S} \mathbf{S}^{-1}\left(\overline{\mathbf{x}}_{1}-\overline{\mathbf{x}}_{2}\right)} \\
& =\left(\overline{\mathbf{x}}_{1}-\overline{\mathbf{x}}_{2}\right)^{t} \mathbf{S}^{-1}\left(\overline{\mathbf{x}}_{1}-\overline{\mathbf{x}}_{2}\right)=D^{2}
\end{aligned}
$$

$D^{2}$ is called the generalized square distance between the centroids of the two groups, and is also referred to as the Mahalanobis distance, in honor of Prasanta Mahalanobis, who introduced it in 1936 [95]. It can also be noted that the maximum of the FLD function, described in Eq. 4.6 is just $T_{\mathbf{a} \text { max }}^{2}=\left[n_{1} n_{2} /\left(n_{1}+n_{2}\right)\right] D^{2}$. Now, we can define the Mahalanobis distance of an individual event from the centroid of the group, e.g. in the two group case:

$$
\begin{aligned}
& D_{1}^{2}=\left(\mathbf{x}-\overline{\mathbf{x}}_{1}\right)^{t} \mathbf{S}^{-1}\left(\mathbf{x}-\overline{\mathbf{x}}_{1}\right) \\
& D_{2}^{2}=\left(\mathbf{x}-\overline{\mathbf{x}}_{2}\right)^{t} \mathbf{S}^{-1}\left(\mathbf{x}-\overline{\mathbf{x}}_{2}\right) .
\end{aligned}
$$

One can also make explicit the difference of variances between the two groups by replacing $\mathbf{S}$ with $\mathbf{S}_{1}$ and $\mathbf{S}_{2}$ in Eq. 4.13 and Eq. 4.14, respectively, or more generally, by writing

$$
D_{m}^{2}=\left(\mathbf{x}-\overline{\mathbf{x}}_{m}\right)^{t} \mathbf{S}_{m}^{-1}\left(\mathbf{x}-\overline{\mathbf{x}}_{m}\right) .
$$

Furthermore, the Mahalanobis distance (as defined in Eq. 4.13 and Eq. 4.14 for the two group case) may be generalized by using the pooled within-groups covariance matrix introduced in Eq. 4.8:

$$
D_{m}^{2}=\left(\mathbf{x}-\overline{\mathbf{x}}_{m}\right)^{t} \mathbf{W}^{-1}\left(\mathbf{x}-\overline{\mathbf{x}}_{m}\right)
$$


However, the use of this definition is only recommended when it is known that all the groups have similar dispersion characteristics. Therefore, the use of the individual sample covariance matrices $\mathbf{S}_{m}$ for each group, as in Eq. 4.15 can be seen as the more general approach.

The meaning of the Mahalanobis distance can be better understood by considering the case of two variables $u$ and $v$ and two groups $G_{1}$ and $G_{2}$. The squared euclidian distance from the centroid of the $m^{t h}$ group is $\left(u-\bar{u}_{m}\right)^{2}+\left(v-\bar{v}_{m}\right)^{2}$. The set of points at a given distance (also known as loci) are circles, as shown in Fig. 4.5 for the two hypothetical groups. If one desires to take into account the variances of each variable,
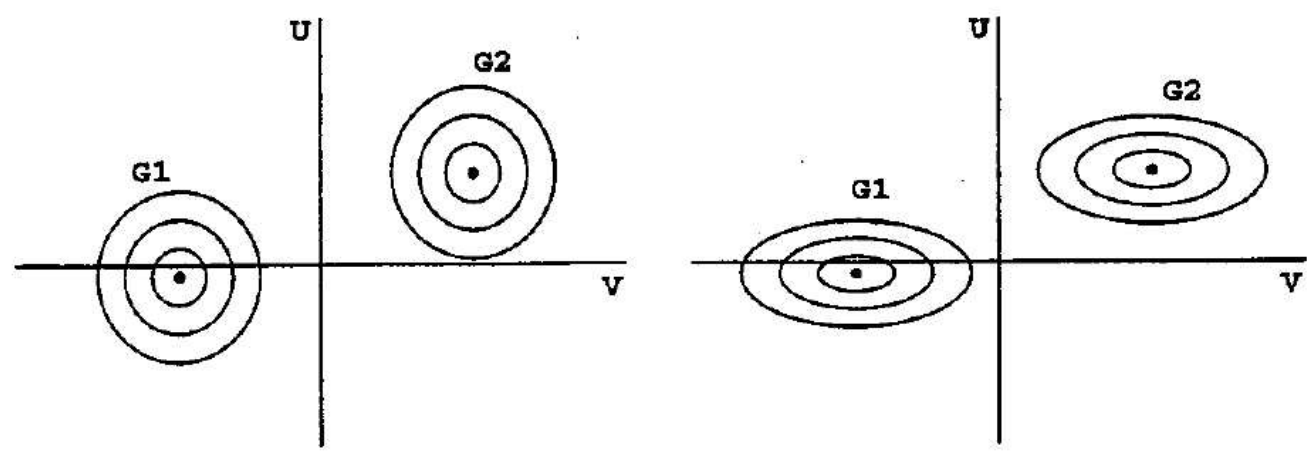

Figure 4.5: The plot on the left depicts loci of equal euclidian distance in the space of two variables $u$ and $v$ for the two groups $G_{1}$ and $G_{2}$. The plot on the right shows loci of equal standard distance.

then the statistical or standard distance $\left(\frac{u-\bar{u}_{m}}{\sigma_{u}}\right)^{2}+\left(\frac{v-\bar{v}_{m}}{\sigma_{v}}\right)^{2}$ must be used. The loci of points are now represented by ellipses, $c f$. Fig. 4.5, unless the variables have identical variances, in which case the standard distance is equivalent to the euclidian distance. The Mahalanobis distance, as defined in Eq. 4.12, becomes then [93]:

$$
D^{2}=\frac{1}{1-r^{2}}\left[\left(\frac{u-\bar{u}_{m}}{\sigma_{u}}\right)^{2}+\left(\frac{v-\bar{v}_{m}}{\sigma_{v}}\right)^{2}-\frac{2 r\left(u-\bar{u}_{m}\right)\left(v-\bar{v}_{m}\right)}{\sigma_{u} \sigma_{v}}\right]
$$

where $r$ is the correlation coefficient between the two variables, given by

$$
r=\frac{1}{n_{m}} \sum_{i=1}^{n_{m}} \frac{\left(u_{i}-\bar{u}_{m}\right)\left(v_{i}-\bar{v}_{m}\right)}{\sigma_{u} \sigma_{v}} .
$$


It is straightforward to notice that if $r=0$, i.e. if there is no correlation between $u$ and $v$, the Mahalanobis distance is simply the standard distance. For $r \neq 0$, the loci are tilted ellipses, with different inclinations for each group if they have distinct covariance matrices (and thus $r$ values), as illustrated in Fig. 4.6.
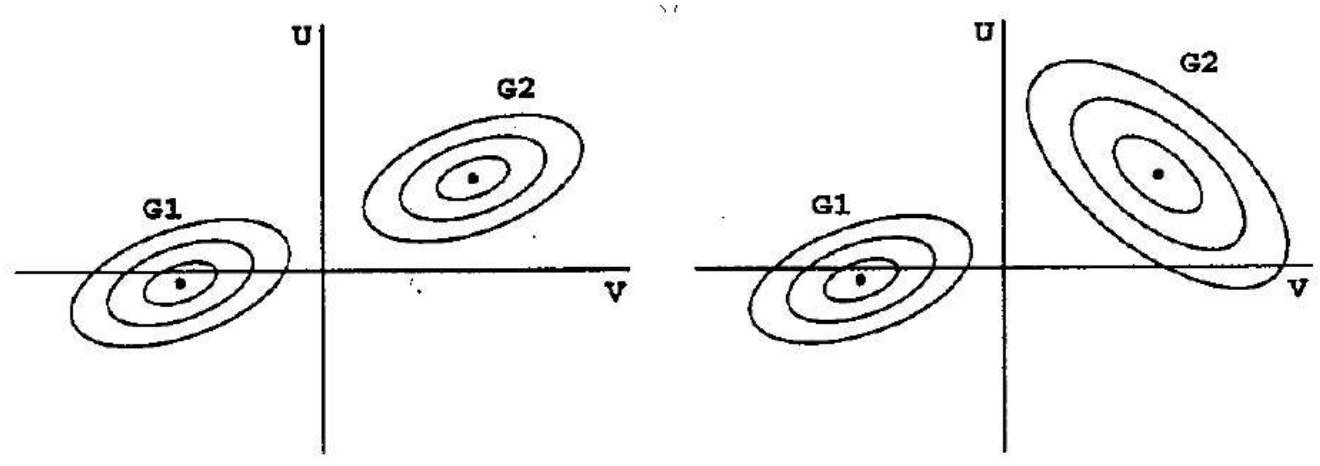

Figure 4.6: Loci of equal Mahalanobis distance using the pooled within-groups covariance matrix (on the left) and using separate covariance matrices for each group (on the right).

\subsubsection{Classification Scores}

If one uses the Mahalanobis distance as a discriminant function, which in the two group case means calculating $D_{1}^{2}$ and $D_{2}^{2}$ for an event, a simple classification criterion is to assign an event to group $G_{1}$ if $D_{1}^{2}>D_{2}^{2}$ and vice-versa. A more sophisticated approach, based on the likelihood concept, consists in defining the function $\mathcal{L}_{m}$ to be the likelihood for an event to belong to the group $G_{m}$ :

$$
\mathcal{L}_{m}=\frac{e^{-D_{m}^{2} / 2}}{\sqrt{2 \pi\left|\mathbf{S}_{m}\right|}} .
$$

This is equivalent to

$$
\ln \mathcal{L}_{m}=-D_{m}^{2} / 2-\ln \left|\mathbf{S}_{m}\right| / 2-\ln \sqrt{2 \pi}
$$

Therefore, in the two group scenario, the classification criterion is now to assign an event to group $G_{1}$ if $\ln \mathcal{L}_{1}>\ln \mathcal{L}_{2}$, equivalent to ${D_{1}^{\prime}}^{2}<{D_{2}^{\prime}}^{2}$, with $D_{m}^{\prime 2}=D_{m}^{2}+\ln \left|\mathbf{S}_{m}\right|$. 
Now we can define a probability, $P_{m}$ that the event belongs to $G_{m}$ :

$$
P_{m}=\frac{\mathcal{L}_{m}}{\sum_{j=1}^{g} \mathcal{L}_{j}},
$$

where $g$ is the total number of groups. For each event we can thus calculate $g-1$ independent a posteriori probabilities or scores. The event is classified in the group that yields the highest score. The $P_{m}$ functions are convenient in that they combine the $D_{1}^{2}, D_{2}^{2}, \ldots, D_{g}^{2}$ distances into discriminant variables with fixed limits from 0 to 1 . The $P_{m}$ are only true probabilities when all the variables from which they are assembled are gaussianly distributed. It should also be noted that this type of discriminant is quadratic in the variables, unlike Fisher's linear discriminant. Any non-gaussianity in the discriminator variables results in less than optimal classification capabilities of the method. In our case, limiting the pool of variables to the ones exhibiting stronger gaussian behavior did not yield any improvement as these variables were not always selected as the ones with the highest discriminant power.

\subsubsection{Stepwise Discriminant Analysis}

In the method discussed in the previous section it is assumed that the best set of discriminator variables, used to form the discriminant function, is known. However, it often occurs that a large number of potential discriminator variables are available, but intuition and ingenuity is not sufficient to determine the best set of variables to assemble the discriminant function. Stepwise discriminant analysis is a technique that allows the determination of the best set of variables in a systematic way.

\section{Forward Selection}

In this type of selection, the first variable entered into the discriminant function is the one that provides the most discrimination between the groups based on a statistical criterion. In the following step, the variable that is entered adds the maximum amount of discriminating power still available to the discriminant function, as measured by the same statistical criterion. This procedure progresses until all the variables are entered or until the remaining variables fail to significantly increase the discriminating power of the discriminant function. 


\section{Backward Elimination}

In the initial step of backward elimination, the discriminant function is formed by all the available variables. At each step, the variable that provides the least amount of decrease in the discriminating power, as measured by a statistical criterion, is removed. The procedure continues until no additional variable can be removed without reducing the discriminating power beyond a given threshold.

\section{Stepwise Selection}

Stepwise selection combines the forward and backward elimination procedures. It starts with no variables in the discriminant function and then, at each step, a variable is either added or removed. A variable already entered in the discriminating variable is removed if its removal does not significantly lower the discriminating power, as measured by the statistical criterion. If no variable is removed than the variable that adds the most discriminating power is entered. The procedure stops when no variable can be removed or added to the discriminant function.

\section{Statistical Criteria}

As mentioned above, the variable selection procedures are based on a measure of the discriminating power by a statistical criterion. The most commonly used criterion is known as Wilks' Lambda and is given by:

$$
\Lambda=\frac{|\mathbf{W}|}{|\mathbf{B}+\mathbf{W}|},
$$

where $|\mathbf{W}|$ is the determinant of the pooled within-group covariance matrix, $c f$. Eq. 4.8 and $|\mathbf{B}+\mathbf{W}|$ is the determinant of the sum of $\mathbf{W}$ with the pooled between-group covariance matrix, $c f$. Eq. 4.9. Wilks' Lambda corresponds to the ratio of the withingroup sum of squares to the total sum of squares. It maximizes the total separation between all groups and can be seen as a direct measure of the proportion of variance between all groups that is unaccounted for by the discriminant function. Therefore, the value of $\Lambda$ will tend to decrease as one includes useful variables in the discriminant and increase or stay the same when one includes, for example, discriminator variables 
that are highly correlated with already added variables. At each step in the selection procedures, the variable included will be the one that results in the smallest value of $\Lambda$, when all the contributions of already included variables are taken into account. Reciprocally, if a variable significantly increases the Wilks' Lambda value, it will be removed from the discriminant function.

Wilks' Lambda can be converted into an F-ratio ${ }^{1}$ using the transformation [93]:

$$
F=\left(\frac{1-\Lambda}{\Lambda}\right)\left(\frac{n-g}{g-1}\right)
$$

where $g$ is the number of groups and $n$ the total number of observations. This transformation is valid for any number of groups, but only if calculated for a single discriminator variable, as it is the case in stepwise procedures. Through this Fdistribution, with $g-1$ and $n-g$ degrees of freedom, it is easy to define a statistical significance (normally $5 \%$ is used as a minimum threshold) for the hypothesis that a variable accounts for the proportion of variance between all the groups not accounted for by already included variables. Therefore, in some applications, the $F$-value and significance are quoted instead of Wilks' Lambda. It should be noted that there are no corresponding transformations that convert Wilks' Lambda in an F-distribution simultaneously for an arbitrary set of $p$-variables and an arbitrary number of groups.

Other possible criteria, such as Rao's V or F-ratios based on Mahalanobis distances are described elsewhere (see Ref. [93]). For the purposes of variable selection for the analysis of this thesis, a stepwise selection procedure was used, based on the Wilks' $\Lambda$ statistical criterion.

\subsubsection{Implementation}

The application of the analysis method delineated above to the reconstructed and reduced MDC samples employed the software package SAS (Statistical Analysis System) version 9, in particular one of its components, the DISCRIM procedure. SAS is a popular multi-platform set of industrial and educational software tools. It is organized

\footnotetext{
${ }^{1}$ The $F$-distribution is a continuous statistical distribution which arises in testing of whether two observed samples have the same variance. If the two samples have $\chi_{m}^{2}$ and $\chi_{n}^{2}$ distributions with $m$ and $n$ degrees of freedom, respectively, the $F$-ratio is $F_{n, m} \equiv \chi_{n}^{2} / \chi_{m}^{2}$. Further details can be found in Ref. [93].
} 
into a number of modules, called products, such as base SAS, SAS/STAT (statistics), SAS/IML (matrices) and SAS/INSIGHT (interactive data analysis). Each one of these products contains several procedures called by statements in SAS macros. The analysis is run via the execution of the program macros within the SAS environment. For this thesis, the typical analysis carried out within SAS consists of:

1. Reading the SAS-formatted text files obtained from the AnaNue ntuples into SAS data libraries.

2. Carrying out any necessary pass(es) over the data libraries using $S Q L$ procedures to e.g. reweight events, eliminate events from the training sample, recast the background groups as a single group, etc..

3. Conducting a preliminary run using the STEPDISC procedure to perform a stepwise selection of discriminator variables.

4. Running the Multivariate Discriminant code using the DISCRIM procedure-the output contains the event classification results and individual probabilities of classification in each group.

5. Using the SAS/INSIGHT module for quick and simple interactive analysis of the results, e.g. the determination of the total weighted number of events classified in each group.

6. Transferring and storing of the identification summary files for posterior generation of PID trees and further analysis.

Fig. 4.7 illustrates the classification output of the DISCRIM procedure for a typical run. The complete analysis procedure will be more thoroughly clarified in the following sections.

\subsection{Background to $\nu_{\mu} \rightarrow \nu_{e}$ Appearance in MINOS}

In experimental high energy physics, the discovery or measurement of a signal invariably implies a prior accurate understanding and quantification of the types of 


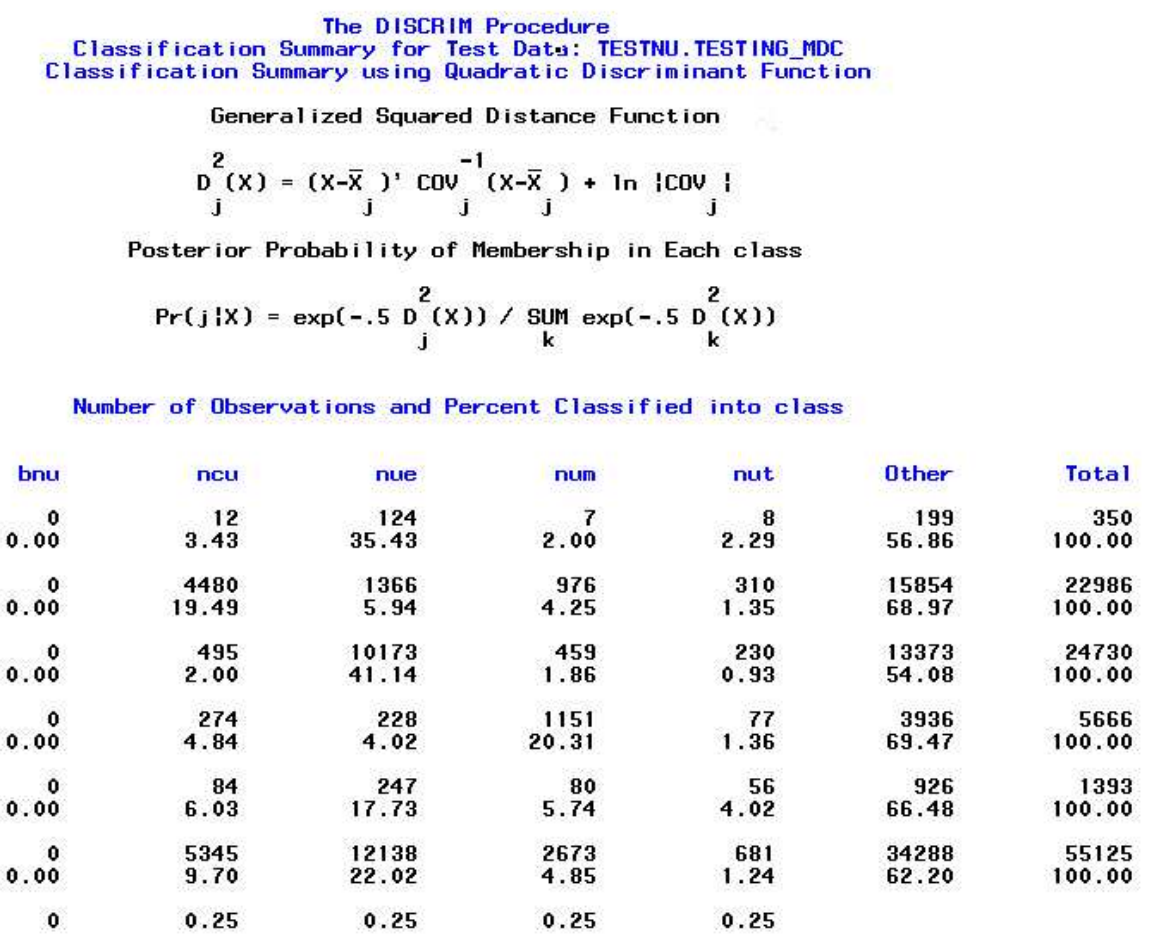

Figure 4.7: An example of the classification output of a typical DISCRIM procedure run. The type of discriminant function is shown, along with the true contents of the sample and the numbers classified into each group.

background that can emulate the signal being sought or measured. This section discusses the various types of background to a potential $\nu_{\mu} \rightarrow \nu_{e}$ appearance signal observation within the MINOS Far Detector.

For the present analysis, the MDC samples are divided in five major classes of events, namely oscillation induced $\mathrm{CC} \nu_{e}, \mathrm{NC}, \mathrm{CC} \nu_{\mu}, \mathrm{CC} \nu_{\tau}$ and beam-induced $\mathrm{CC}$ $\nu_{e}$ events. The first class corresponds to the signal we wish to measure, whereas the latter four classes correspond to potential backgrounds. The MDA procedure uses the first four classes as its classification groups. No event is classified into the fifth class, beam-induced $\nu_{e} \mathrm{CC}$ events, because these interactions look identical to the ones originated by the signal being measured. Let us now take a closer look at each type of background. 


\subsubsection{Neutral Current Events}

Neutral current interactions constitute the main source of background, in particular events with an energetic incoming neutrino that induces $\pi^{0}$ production, for which a large fraction of the energy deposited is electromagnetic, e.g. :

$$
\nu_{\mu}+N \rightarrow \nu_{\mu}+p+\pi^{0} \searrow_{\gamma \gamma} .
$$

The MINOS detectors coarse pitch severely constrains the ability to resolve the two photons originating from the $\pi^{0}$ decay. This type of background is virtually irreducible. An example is provided in Fig. 4.8.
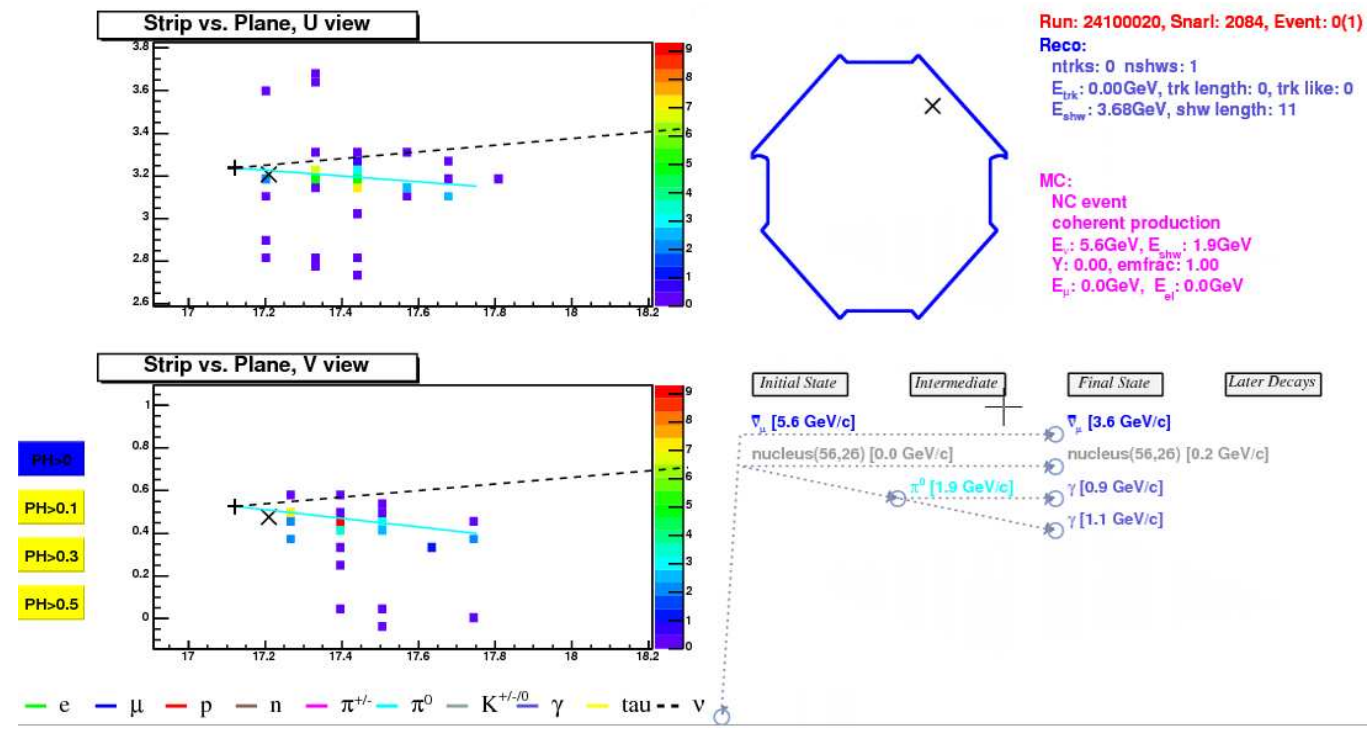

Figure 4.8: A background CPP (coherent pion production) NC event. The graphs on the left show the strip representations of the event with the truth particle vectors superimposed. On the right, the upper plot shows the vertex location in the detector and summarizes the reconstruction and truth information, whereas the bottom plot shows a diagram of the interaction extracted from truth.

\subsubsection{Charged Current $\nu_{\mu}$ Events}

The $\nu_{e}$ appearance signal may also be simulated by DIS (deep inelastic scattering) or RES (resonance production) charged current $\nu_{\mu}$ interactions with large values of 
hadronic inelasticity ${ }^{2} y$. Therefore, in high $y$ charged current $\nu_{\mu}$ events, described by

$$
\nu_{\mu}+N \rightarrow \mu^{-}+X^{+,++},
$$

where $X$ denotes the hadronic final state, the emerging muon has low momentum and the resulting short track can be hidden by the hadronic shower. The electromagnetic shower fitting variables help the elimination of this type of background, by means of the poor fitting results for hadronic showers. However, if $\pi^{0}$ production occurs and the $\pi^{0}$ carries enough energy, the resulting electromagnetic shower could closely mimic an electron shower, rendering its discrimination as difficult as for the type of background alluded to in the previous sub-section. An illustration of this background is shown in Fig. 4.9. An additional source of background arises from low $y \mathrm{CC} \nu_{\mu}$ events close to the edges of the detector, where the muon leaves the detector shortly after the interaction occurs. This can be dealt with through the use of judicious containment cuts, to be discussed below.
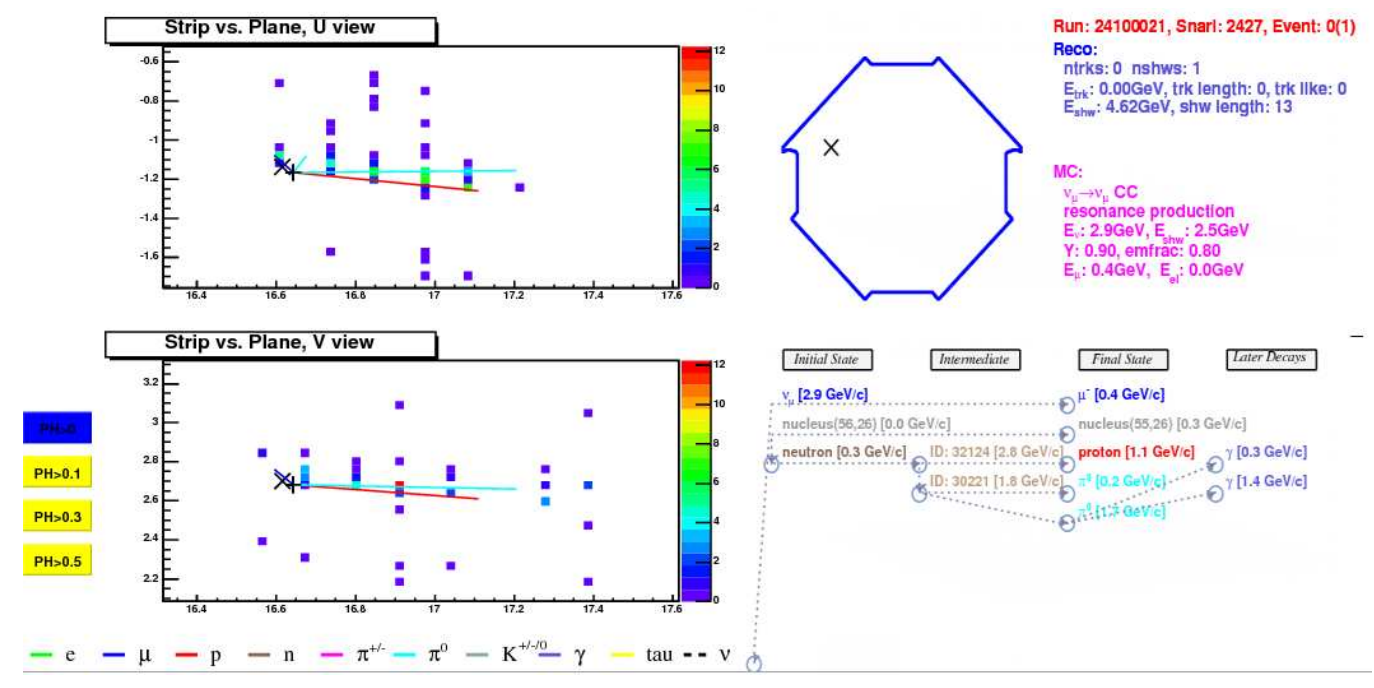

Figure 4.9: A background RES CC $\nu_{\mu}$ event. In this high $y$ event the muon track is invisible and the produced $\pi^{0}$ carries enough energy to originate an electromagnetic shower easily confused with an electron-induced shower.

\footnotetext{
${ }^{2}$ Inelasticity is a variable used in the description of the behavior of particles in inclusive reactions. It is given by $y=\frac{E_{t o t}-E}{E_{t o t}}$, where $E_{t o t}$ is the energy of the incoming particle(s) and $E$ the energy of a given particle. For our case, we make use of the inelasticity of the hadronic final state defined as $y=\frac{E_{h a d}}{E t o t} . y$ is not to be confused with the rapidity kinematic quantity.
} 


\subsubsection{Charged Current $\nu_{\tau}$ Events}

The CC $\nu_{\tau}$ interactions at the Far Detector result from $\nu_{\mu} \rightarrow \nu_{\tau}$ oscillations which are progessively suppressed for higher energy values of the incoming neutrino (e.g. less than $20 \%$ of $\nu_{\mu}$ oscillate for $E_{n u}>5 \mathrm{GeV}$, for $\Delta m_{23}^{2}=2.5 \times 10^{-3} \mathrm{eV}^{2}$ and maximal mixing). Given the additional relatively high energy threshold for $\tau$ production $(\sim$ $3.5 \mathrm{GeV}$ ), the number of $\mathrm{CC} \nu_{\tau}$ events occurring at the Far Detector will be inherently small. The background to $\nu_{e}$ appearance arises in CC $\nu_{\tau}$ interactions where the $\tau$ decays into an electron (17.8\% B.R.) or into a low multiplicity hadronic system with $\pi^{0}$ production (36.9\% B.R.),

$$
\nu_{\tau}+N \rightarrow p+\tau^{-} \searrow\left\{\begin{array}{l}
\nu_{\tau}+\overline{\nu_{e}}+e^{-} \\
\nu_{\tau}+h^{-}+\pi^{0},
\end{array}\right.
$$

where $h^{-}$stands for $\pi^{-}$or $K^{-}$.

Because of the aforementioned limited statistics, this is the less relevant source of background to $\nu_{\mu} \rightarrow \nu_{e}$ appearance. An example of a CC $\nu_{\tau}$ interaction with production of an electron is presented in Fig. 4.10.

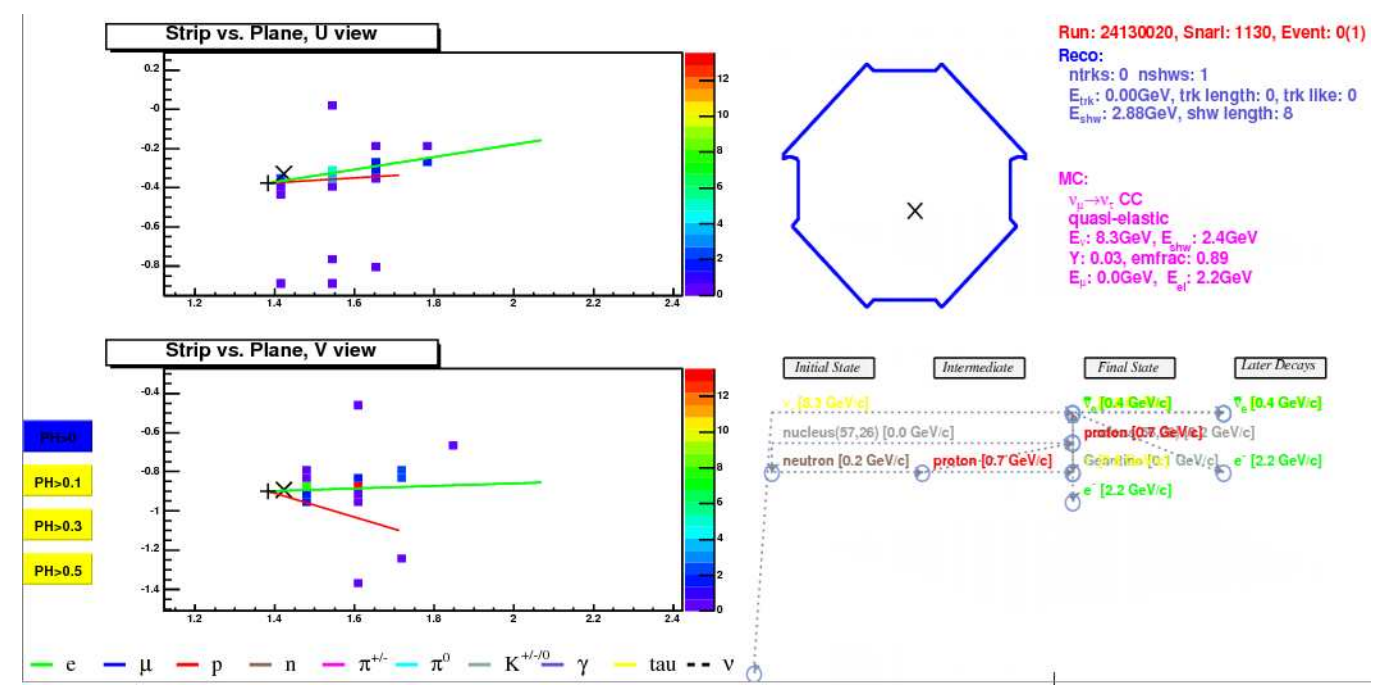

Figure 4.10: A background $\mathrm{QE} \mathrm{CC} \nu_{\tau}$ event. A QE interaction of a reasonably energetic parent neutrino produces a $\tau$ that promptly decays into an electron, producing a clear electromagnetic shower. 


\subsubsection{Beam-induced Charged Current $\nu_{e}$ Events}

This background originates from contamination of the NuMI beam by $\nu_{e}$ particles $(\sim 0.5 \%)$. These result predominantly from muon decays (recall that the MINOS horns primarily focus the secondaries $\pi^{+}$and $K^{+}$),

$$
\mu^{+} \rightarrow e^{+}+\nu_{e}+\overline{\nu_{\mu}},
$$

and $K_{e 3}$ decays,

$$
K^{+} \rightarrow \pi^{0}+e^{+}+\nu_{e} .
$$

Fig. 4.11 illustrates the expected flux of intrinsic $\nu_{e}$ in the NuMI beam with the component originated from $K^{+}$decay made explicit.
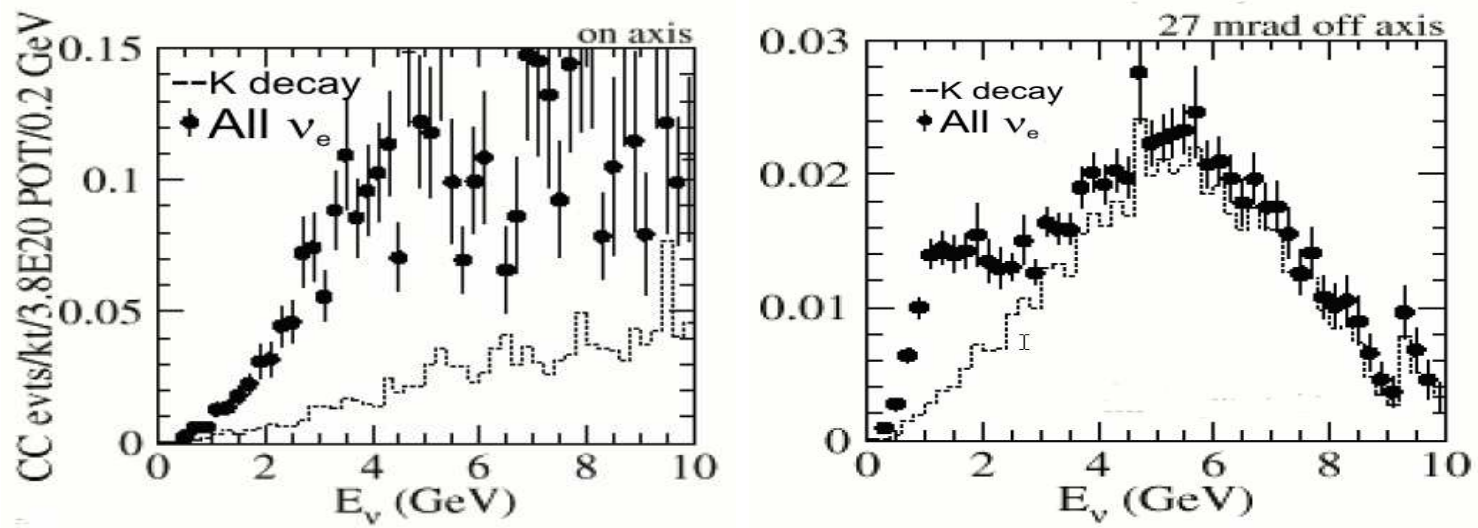

Figure 4.11: Expected flux at the Far Detector for the NuMI beam $\nu_{e}$ component at the nominal on-axis position (left plot) and, shown for comparison purposes, at an hypothetical position 27 miliradians offset from the beam axis (right plot). The portion of $\nu_{e}$ resulting from $K_{e 3}$ decays is shown explicitly. Plots courtesy of S. Kopp.

The beam $\nu_{e}$ interact in the MINOS detectors in the same way the signal $\nu_{e}$ would. However, their energy distribution is peaked at higher values than the one obtained by oscillation of the beam $\nu_{\mu}$ spectrum, as illustrated in Fig. 4.12. Furthermore, the beam $\nu_{e}$ contamination should be estimated with considerable precision (possibly better than 10\%) using the Near Detector data. In our case, the number of beam $\nu_{e}$ is estimated by running the same discriminant applied to the Far Detector on the Near Detector MDC samples. It should be noted that due to the higher transverse 

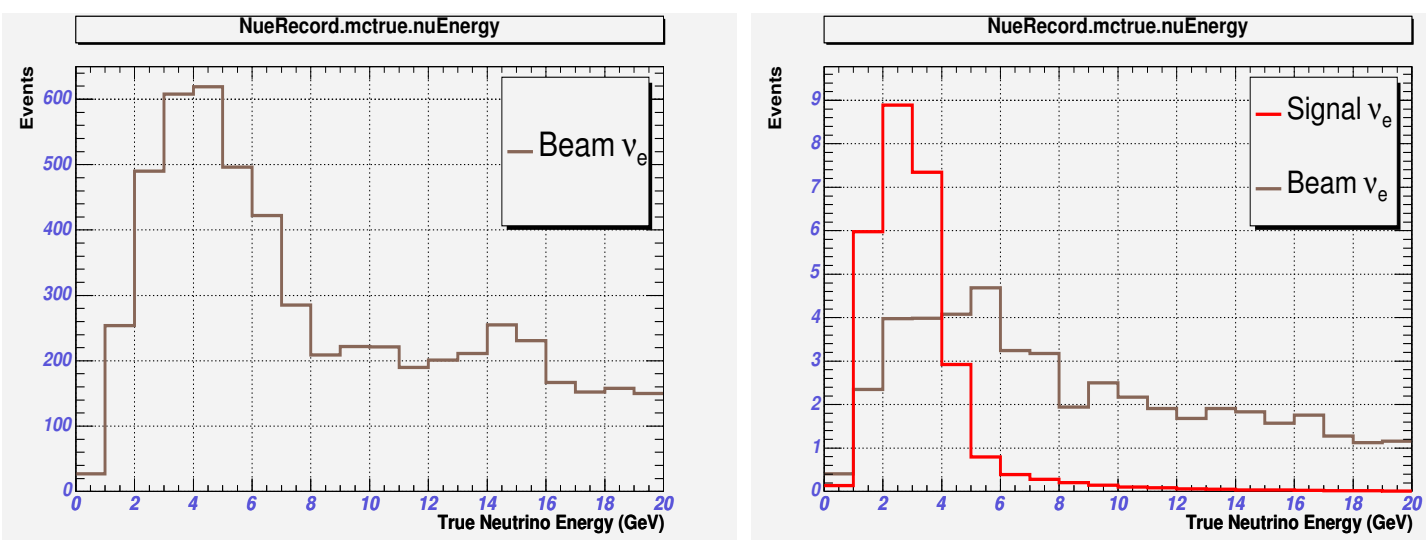

Figure 4.12: Comparison between the energy spectrum expected at the Near (left plot) and Far (right plot) Detectors for signal and beam $\nu_{e}$ events. The signal $\nu_{e}$ distribution is computed using the oscillation parameters $\Delta m_{23}^{2}=2.5 \times 10^{-3} \mathrm{eV}^{2}$, $\sin ^{2} 2 \theta_{23}=1.0$ and $\left|U_{e 3}\right|^{2}=0.01$.

momentum of the secondary $K^{+}$with respect to the $\pi^{+}$, a considerable number of the $K^{+}$-induced $\nu_{e}$ events will not reach the Far Detector (the expected number at the Far Detector is shown in Fig. 4.11, where it can be seen the $K^{+}$-induced component has a much wider angular distribution) and therefore extrapolation of the observed $\nu_{e}$ at the Near Detector to the Far Detector has to be done carefully. A typical beam-induced $\nu_{e}$ event is shown in Fig. 4.13.

In the next section, a discussion of cuts that contribute to a preliminary background reduction will be presented.

\subsection{Analysis Samples}

In order to carry out the MDA analysis, the subdivision of the MDC samples is necessary. The first subsample is the reference set of observations that, to the best of our understanding, reflects the characteristics of each of the groups in which we wish to classify the Mock Data. This sample is composed of a number of MDC Far Monte Carlo files, including some of the auxiliary files alluded to in Section 3.1. The beam and auxiliary files are assembled together in equal proportions. We refer to this set as the training sample. 


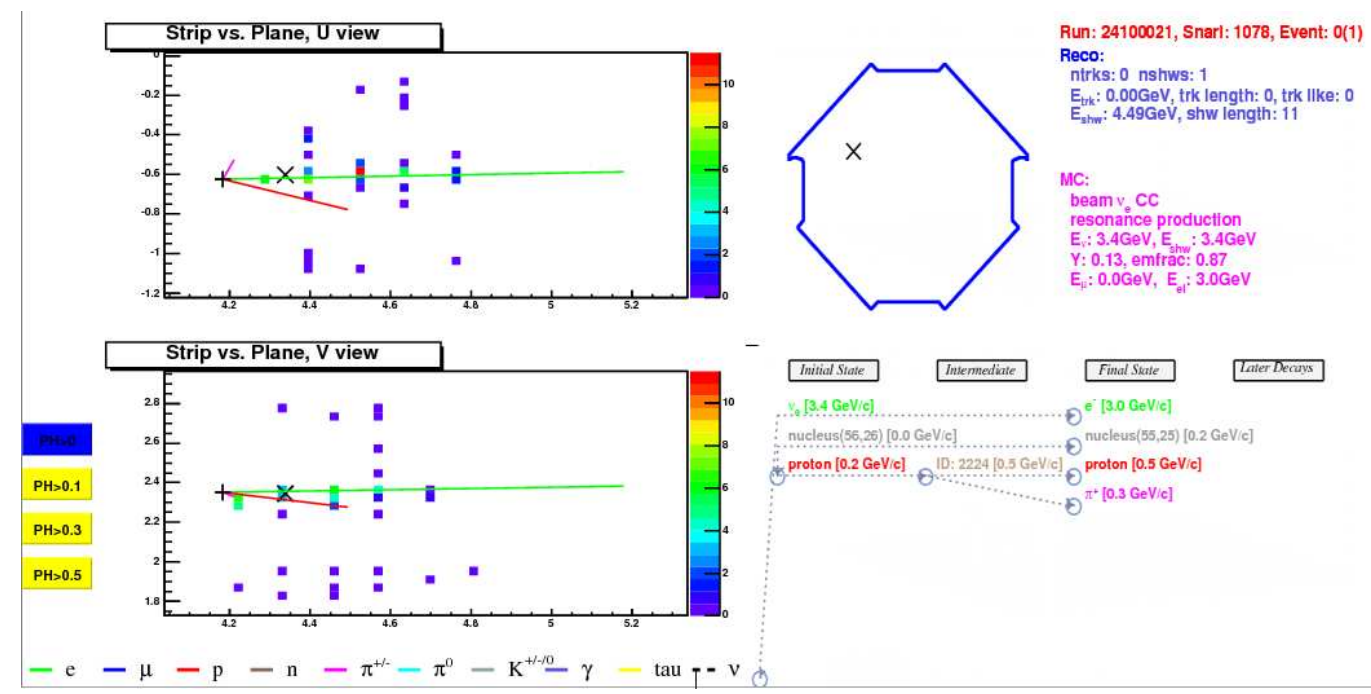

Figure 4.13: A background beam-induced RES CC $\nu_{e}$ event. The beam induced $\nu_{e}$ events peak at higher energies than the $\nu_{\mu}$ in the beam spectrum and thus, than the potential $\nu_{e}$ appearance events. Therefore, they are more prone to producing DIS and RES interactions. However, for low $y$ events, such as this one, the secondary electron still carries most of the energy and is forward enough that it may be easily confused with a signal QE CC $\nu_{e}$ event.

As a means of validating and understanding analysis performance, a test sample is defined, with size identical to the training sample, equal proportions of beam and auxiliary files, but without any overlapping events with the training sample. The multivariate discriminant built by running on the training sample is run without modification on the test sample, ensuring an unambiguous assessment of analysis performance. Since the test sample consists of MDC Far Monte Carlo files, the truth information is available and can be used to quantify classification quality and produce analysis efficiency studies. The MDA obtained from the training sample is also run on another test sample, composed of the totality of MDC Near Monte Carlo files. No training is performed on Near Detector files, as the characterizing features of each type of events should be identical to the ones found in the Far Detector, so as to ensure a meaningful extrapolation of the Near Detector results to the Far Detector.

Finally, there are three Mock Data samples, for which truth is unknown, obtained from the MDC Challenge sets. One comprises a single Far Detector Mock Data 
file, corresponding to an intensity of $7.4 \times 10^{20}$ POT, about 2 years of MINOS data taking; the second one consists of three Far Mock Data files, thus corresponding to $22.2 \times 10^{20}$ POT and 6 years of operation; the third encompasses the complete set of Near Mock Data files, corresponding to an intensity of $3.2 \times 10^{18}$ POT ( $~ 3$ days of running), and is used to constrain the fitting of $\nu_{\mu} \rightarrow \nu_{e}$ oscillation models to the Mock Data, to be discussed in the next Chapter.

Table 4.1 displays an overview of the components of each analysis sample.

\begin{tabular}{|c|c|c|c|c|}
\hline Analysis Sample & Beam & $\nu_{e}$ flavor & $\nu_{\tau}$ flavor & POT/file \\
\hline \hline Training & 19 & 19 & 19 & $6.5 \times 10^{20}$ \\
\hline Far Test & 19 & 19 & 19 & $6.5 \times 10^{20}$ \\
Near Test & 229 & - & - & $1.33 \times 10^{16}$ \\
\hline Far Mock Data (2 y) & 1 & - & - & $7.4 \times 10^{20}$ \\
Far Mock Data (6 y) & 3 & - & - & $7.4 \times 10^{20}$ \\
Near Mock Data & 244 & - & - & $1.33 \times 10^{16}$ \\
\hline
\end{tabular}

Table 4.1: Composition in terms of number of MDC files of each analysis sample used in the MDA analysis.

\subsection{Sample Cuts}

The application of cuts to the analysis samples serves multiple purposes. One of the most relevant is to ensure consistency between the Near and Far distributions of each discriminator variable. This allows for a meaningful extrapolation of the Near Detector analysis results to the Far Detector, further constraining oscillation model fitting, as we will see later. The sample cuts are also used to focus the MDA on the potentially more significant discrimination region by performing an a priori removal of obviously uninteresting events, e.g. events with long tracks, and minimizing the background sources, while keeping the largest possible amount of signal intact. Finally, all the cuts to be described have the indirect effect of reducing significantly the size of the samples to be operated on, thus drastically shortening computation times and the size of the output files to be carried through the full analysis chain. The precise values for the cuts described below were obtained in a study performed at Harvard. It consisted in measuring the evolution of a quality criterion, the figure 
of merit, defined as

$$
F O M=\frac{\text { Signal }}{\sqrt{\sum \text { Background }}},
$$

while varying the cuts applied to some key quantities, such as reconstructed energy, event length, etc.. The final cuts obtained were adopted for both the Harvard Decision Tree analysis and the Tufts Multivariate Discriminant analysis.

\section{Energy Cuts}

The application of lower and upper energy cuts eliminates those events for which too little energy is deposited in the detector to allow any unambiguous classification, and also eliminates events in the high energy range where there is a small chance of finding signal $\nu_{e}$ events. In addition, the high energy cuts remove a considerable amount of beam-induced $\nu_{e}$ background. These cuts further contribute to the harmonization of the distribution of discriminator variables between the Near and Far detectors. An example is shown in Fig. 4.14.

Quantitatively, the energy cuts are:

i) High energy cut: Accept events with total reconstructed event energy $<150 \mathrm{MEU}$ $(\sim 6 \mathrm{GeV})$.

ii) Prong cut: Individual track or shower pulse height $>5000 \operatorname{SigCor}^{3}(\sim 350 \mathrm{MeV})$.

The effects of these cuts on the data samples are summarized in Table 4.2.

\section{Length Cut}

Essentially aimed at eliminating $\mathrm{CC} \nu_{\mu}$ events from the sample, while preserving events where shower characteristics are dominant:

i) Track length cut: Accept Events with reconstructed track for which track length $<18$ planes.

\footnotetext{
${ }^{3}$ Energy unit used in MINOS that includes corrections for strip-to-srip response differences.
} 

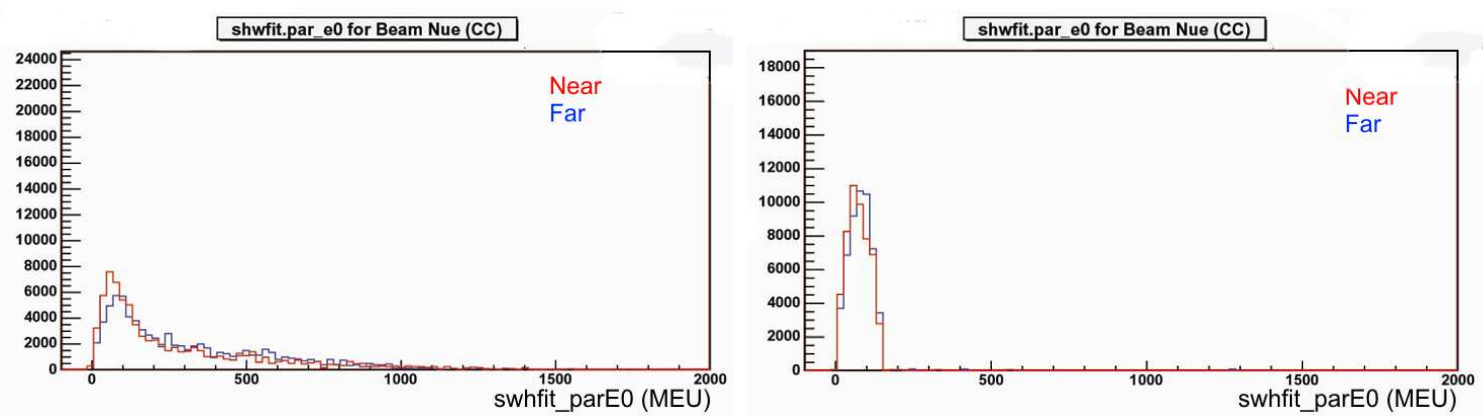

Figure 4.14: Comparison of a variable distribution for Near and Far events before and after a high energy cut. On the left, the Near and Far distributions of the variable shwfit_parE0 for beam $\nu_{e}$ events are shown without any cuts applied. On the right, the same distributions are shown after a high energy cut is applied.

\section{Fiducial and Containment Cuts}

These cuts attempt to minimize potential backgrounds derived from distortion by truncation of events whose vertex is placed close to the edges or the coil holes of the detectors. They also contribute to improve consistency between the Near and Far analyses. The containment cuts make use of the 3D hit representation of the event to evaluate confinement. The cuts used in the Far detector are the following:

i) The event vertex must be contained in the fiducial volume ( $\sim 84 \%$ of the total volume):

(a) $0.35 \mathrm{~m}<z<14.57 \mathrm{~m} \vee 16.2 \mathrm{~m}<z<29.62 \mathrm{~m}$, where $z$ represents the longitudinal coordinate along the beam direction.

(b) $0.4 \mathrm{~m}<r<3.87 \mathrm{~m}$, where $r$ is a radial coordinate perpendicular to the beam direction. The lower limit avoids the coil hole whereas the upper limit eliminates events close to the edges of the detector.

ii) Full event containment in the detector is required, with the containment region identical to the fiducial region defined above.

In the case of the Near detector, the cuts used were:

i) The event vertex must be contained in the fiducial region: 
(a) $0.5 \mathrm{~m}<z<6.5 \mathrm{~m}$, where $z$ represents the longitudinal coordinate along the beam direction.

(b) $0.0 \mathrm{~m}<r<1.0 \mathrm{~m}$. where $r$ is a radial coordinate around the beam center, located at $(1.49 \mathrm{~m}, 0.140 \mathrm{~m})$ in the Near Detector transverse coordinates $(x, y)$. This cut discards events with the vertex close to the coil.

ii) Full containment in $z$ within the Calorimeter region of the Near Detector is required; the other regions of the Near Detector are not used in the analysis.

\section{Result Summary}

The results of applying the cuts to the Far Test sample are condensed in Table 4.2. For comparison with the results to be presented in later sections, the numbers weighted by oscillation probability and exposure are also shown, along with the corresponding FOM obtained.

\begin{tabular}{|c|c|c|c|c|c|c|}
\hline & $\mathrm{CC} \nu_{e}$ & $\mathrm{NC}$ & $\mathrm{CC} \nu_{\mu}$ & $\mathrm{CC} \nu_{\tau}$ & Beam CC $\nu_{e}$ & $\mathrm{FOM}$ \\
\hline \hline Total & 63875 & 46376 & 63388 & 10011 & 1888 & - \\
Weighted & 27.61 & 1154.78 & 3375.91 & 32.63 & 70.31 & 0.41 \\
\hline Energy Cuts & 38095 & 34462 & 39989 & 3053 & 570 & - \\
Weighted & 25.85 & 854.63 & 1702.18 & 24.21 & 21.02 & 0.51 \\
\hline Length Cut & 37114 & 32934 & 11142 & 2338 & 552 & - \\
Weighted & 25.40 & 815.71 & 441.05 & 20.04 & 20.35 & 0.70 \\
\hline Fid. \& Cont. Cuts & 24730 & 22986 & 5666 & 1393 & 350 & - \\
Weighted & 17.51 & 568.91 & 186.43 & 13.21 & 12.90 & 0.63 \\
\hline
\end{tabular}

Table 4.2: Number of surviving events in the Far Test sample for each class as successive cuts are applied. Numbers weighted with oscillation parameters $\Delta m_{23}^{2}=2.5 \times$ $10^{-3} \mathrm{eV}^{2}, \sin ^{2} 2 \theta_{23}=1.0,\left|U_{e 3}\right|^{2}=0.01$ and an intensity of $9.25 \times 10^{20}$ POT, equivalent to a 10 kt-year exposure (with $84 \%$ fiducial volume), are also displayed.

The table shows a dramatic reduction in the number of CC $\nu_{\mu}$ events (94\%) as well as of the beam-induced $\mathrm{CC} \nu_{e}$ events $(82 \%)$, while $\sim 65 \%$ of the signal remains intact. The number of $\mathrm{NC}$ events is reduced by $50 \%$. A decrease in value of the FOM due to the application of fiducial and containment cuts is noticeable. As mentioned, these cuts are necessary to eliminate the background from truncated events, as well as to avoid distorted signal events that could seriously undermine the discriminant function 
calculation from the reference or training sample. The loss incurred by applying the cut is largely compensated by a higher classification quality.

Before running the MDA procedure, we have to choose the variables that will coalesce into the discriminant functions. The selection method is described in the next section.

\subsection{Variable Selection}

The AnaNue ntuples contain entries for more than 350 variables. Besides the analysis variables previously discussed, several auxiliary reconstruction variables, along with truth variables (in the case of the MC sample) are included. The choice of variables from AnaNue is registered on an input parameter file containing the names of variables to be read out.

The first step in selecting variables for analysis concerns the generation of subsets of the original AnaNue ntuples including only the relevant variables described in Appendix B with any necessary cuts applied. Since the analysis is to be run on both the Near and Far MDC samples, the Near and Far distributions of each discriminator variable should be as close as possible. The consistency check can be achieved using one of the NueAna modules, which reads a parameter file and the full or abridged AnaNue ntuples and produces a Near/Far comparison plot for each variable found therein. A visual evaluation of each plot is done and the variables showing sensible discrepancies between detectors are eliminated. At this stage, another NueAna module is used to read the ntuples, using the parameter file as an input, and to produce a data file in a SAS-readable format. The main component of variable selection may now take place.

Using the STEPDISC procedure of SAS, a stepwise selection algorithm following the description in Section 4.1.5 is applied to $\sim 150$ surviving variables. The method returns a list of variables ordered by discriminating power, according to the Wilks' $\Lambda$ criterion, as depicted in Fig. 4.15.

Although a number of steps equal to the number of variables is used with the stepwise method, typically only $2 / 3$ of the variables are present in the output list, as 
The STEPDISC Procedure

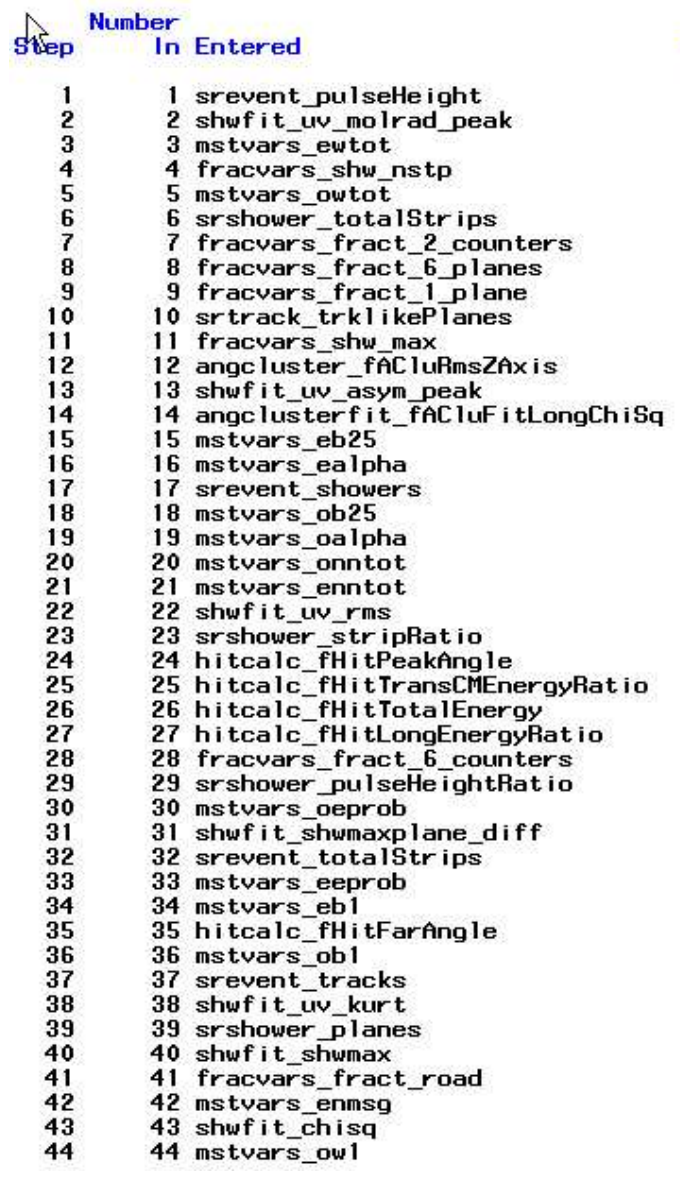

Removed

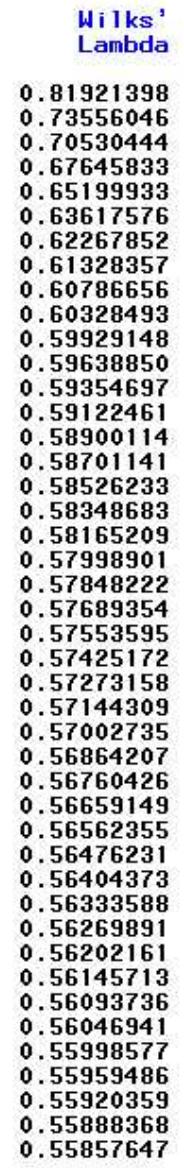

0.73556046

.70530444

0.63617576

.62267852

0.60786656

0.60328493

59929148

4

0.59122461

.58900114

0.58701141
0.58526233

0.58348683

.58165209

57689354

.57553595

0.57273158

0.57144309

.57002735

.56864207

.56659149

.56562355

56404373

.56333588

.56269891

202161

56093736

56046941

.55959486

0.55857647

Figure 4.15: An example of the output of SAS's STEPDISC procedure. The output lists the input variables in descending discriminating power, as measured by the Wilks' $\Lambda$ criterion. The best 19 variables are used to determine the discriminant function. For brevity, only the first 45 steps are shown.

none of the remaining variables adds significant discriminant power. Using subsets containing as many as 30 and as little as 10 of the best variables in the stepwise list, preliminary DISCRIM procedures are run on the training sample. The set of variables that yields the highest value of $F O M$ is chosen for the final classification steps. It was verified that the set of 19 best variables yields the best results. These can be seen by the small decrements in the value of Wilks' $\Lambda$ after the first 20 discriminator variables in the list. The fact that many variables are necessary can be attributed to 
the complexity of classifying the samples in 4 different groups, implying an accrued difficulty in defining one or two variables that strongly discriminate between all of the groups. On the other hand, using a larger number of variables is also unfavorable due to cumulation of the non-gaussianity of the variables, which increasingly biases the MDA method towards smaller separation between groups.

Finally, some care must be exercised when handling missing values. In the AnaNue ntuples, it often happens that some variables will have missing values. Missing values occur e.g. in shower fitting related variables when the fit does not converge or in 3D hit related variables when not enough contiguous strips needed to assemble the hits can be found in an event. The DISCRIM procedure is ill-equipped to handle events with variables that have missing values, as it will simply disregard such events and ignore the remaining variables. To counteract this limitation, a common technique is to impute the missing value of a variable with the mean value of that variable calculated over the full sample ( $c f$. Section 9.6 of Ref. [96]). In this way, a missing value does not bias classification towards any preferential direction, and the DISCRIM procedure operates properly.

\subsection{Analysis Procedure}

In this section, the classification results obtained trough MDA are given and the performance of the analysis methods employed is reviewed.

The complete MDA analysis chain from the AnaNue ntuples to the PID ntuples, of which several components have been discussed throughout this chapter, can be summarized as follows:

1. Definition of analysis samples.

2. Elimination of ancillary variables, such as those related to truth information or run number.

3. Iteration on graphs of relevant discriminator variables output to determine appropriate sample cuts. 
4. Visual scanning of graphs of potential discriminator variables produced for the sample with cuts to decide on a final set of variables to enter the stepwise selection.

5. Use of SAS to perform stepwise selection of the set of discriminator variables to be used to calculate the multivariate discriminant function.

6. Running of MDA on the training sample using SAS, varying probability thresholds to maximize the FOM value.

7. Application of the obtained discriminant to the classification of events on the remaining analysis samples.

8. Reading of the classification output into PID ntuples, which are employed in the assessment of classification performance through efficiency-purity and background studies.

9. Fitting of the Mock Data samples using the PID ntuples (see Chapter 5).

We will focus our attention on items $6-8$, since the remaining items have already been discussed or will be described in the next chapter.

\subsubsection{MDA Training}

As mentioned in Section 4.4, to perform classification using MDA, it is necessary to provide the method with a training sample containing events representative of the different groups we wish use for purposes of classification. By employing different types of training samples in the construction of the multivariate discriminant, it is possible to improve the identification of events that best describe the signal we wish to measure and/or focus on the most difficult distinctions between events to improve background rejection. Different types of training samples combined with MDA runs with different numbers of groups were tested in this analysis. The methods that yielded the best results in achieving $\nu_{e}$ signal separation were the following:

A. Classification into 4 groups: signal $\nu_{e}, \mathrm{NC}, \mathrm{CC} \nu_{\mu}, \mathrm{CC} \nu_{\tau}$; 
B. classification into 4 groups with the signal $\nu_{e}$ component of the training sample composed exclusively of quasi-elastic CC $\nu_{e}$, so the groups become: $\mathrm{QE} \mathrm{CC} \nu_{e}$, $\mathrm{NC}, \mathrm{CC} \nu_{\mu}, \mathrm{CC} \nu_{\tau}$;

C. classification into 2 groups and $\mathrm{QE} \mathrm{CC} \nu_{e}$ training, where the three non-signal categories form a potential background group, so that we have: QE CC $\nu_{e}$, Background ( $\mathrm{NC}, \mathrm{CC} \nu_{\mu}$ and $\mathrm{CC} \nu_{\tau}$ combined).

D. classification into 2 groups with training sample composed exclusively of QE CC $\nu_{e}$ and $\mathrm{NC}$ events, so the groups are: $\mathrm{QE} \mathrm{CC} \nu_{e}, \mathrm{NC}$.

The discriminant functions are determined by running on the Training sample without any weights applied. To estimate the event proportions expected to be found in the data, oscillation probability weights and exposure weights are applied after classification. In determining the best method to apply, both Super-K and the MINOS CC group MDC values for the oscillation parameters were used. Exposures of 2 and 2.5 years of running, corresponding to $7.4 \times 10^{20}$ POT and $9.25 \times 10^{20}$ POT, respectively, were considered.

The results of classification with these different approaches are summarized in the next sections.

\subsubsection{MDA Threshold Determination}

The multivariate discriminant used for classification in the analysis samples was described in Section 4.1.4 and is constructed without pooling among groups, i.e. each group's individual covariance matrix is used instead of a common one. Since we wish to perform classification into four groups, the output of the SAS DISCRIM procedure contains four different probability values, though only three of them are independent.

The typical probability distributions obtained by running MDA to perform classification on event samples are illustrated in Fig. 4.16 and Fig. 4.17.

The severe classification overlap between different types of event can be also demonstrated by plotting the event scores of belonging to one group against the scores of belonging to other groups. The three graphs that can be generated by plotting against the probability of belonging to the signal $\nu_{e}$ group are shown in Fig. 4.18. 


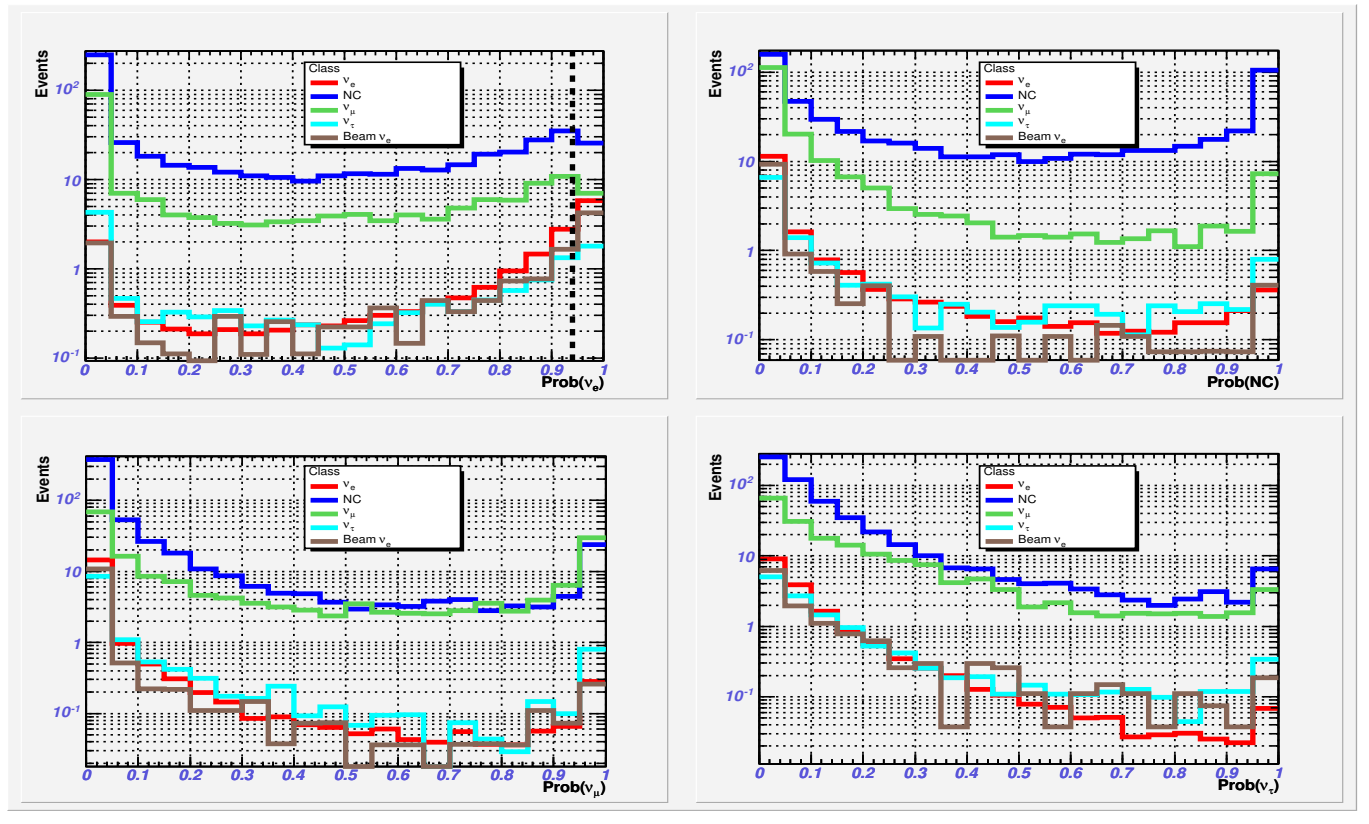

Figure 4.16: An example of the four probability or score distributions for different classes of events obtained by application of MDA to the Test sample with cuts. The signal $\nu_{e}$ events correspond to the red histogram. The plots are shown with logarithmic scale to facilitate the distinction of the several curves. The upper left plot shows the identification probability for an event of belonging to the signal $\nu_{e}$ category. The black dashed line represents the lower probability threshold that maximizes the FOM quantity in this case. The distributions are weighted by an oscillation probability at SuperK best fit values and by a 2.5 year exposure, as described in the previous section.

Looking at these distributions it is clear that one can increase the relative amount of signal against the amount of background. This can be achieved through application of a probability threshold cut, such that any event that does not have a minimum signal $\nu_{e}$ score is discarded. The optimum cut can be determined by plotting the FOM value obtained for different values of the threshold cut, where FOM is, as mentioned before, given by:

$$
F O M=\frac{\text { Signal }}{\sum \text { Background }} .
$$

The FOM vs. threshold plot defines a curve, as depicted in Fig. 4.19, and the maximum value of the curve corresponds to the optimum threshold cut in the training sample. It does not necessarily correspond to the optimum cut for the test samples, 

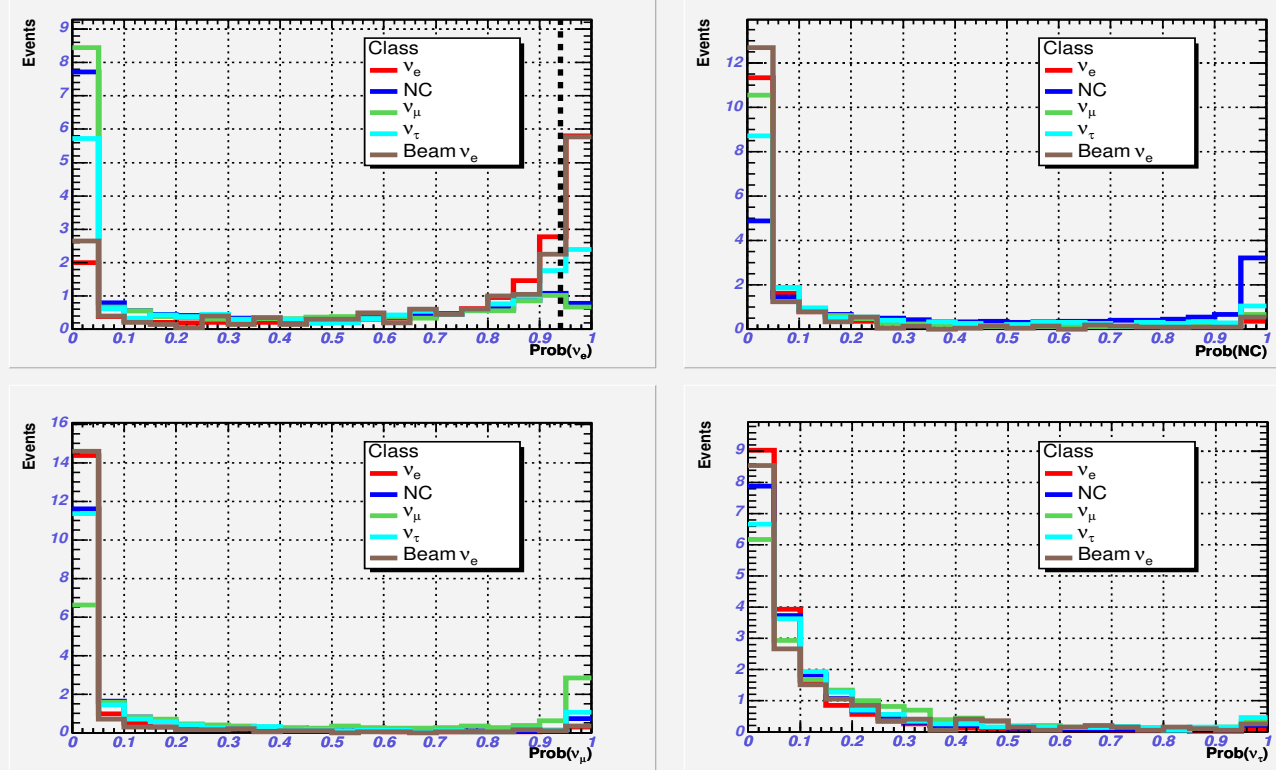

Figure 4.17: This set of plots is identical to the set presented in Fig. 4.16, except no logarithmic scale is applied and the different histograms are normalized to the signal $\nu_{e}$ histogram, so that the relative proportions of events classified into some probability regions is readily apparent. In the upper left plot it is evident that the large majority of $\nu_{e}$ events are assigned a very high score for belonging to the $\nu_{e}$ signal group.

but it constitutes a reliable approximation. Early testing of the MDA analysis, where several small non-overlapping test samples underwent classification, indicated that the variation of the procedure results with input sample is lower than $3 \%$.

The FOM curve application is similar to the concept of the $R O C$ (Receiver Operating Characteristic) diagram, also know as Neyman-Pearson curve, which plots the true positive rate (in our case, fraction of $\mathrm{CC} \nu_{e}$ correctly identified) against the false positive rate (fraction of non-signal events classified as $\mathrm{CC} \nu_{e}$ ). The larger the area underneath the ROC curve of a method, the better classifier the method is. The ROC curve corresponding to our application of the MDA method is shown in Fig. 4.20. See Ref. [97] for further details on ROC analysis. 

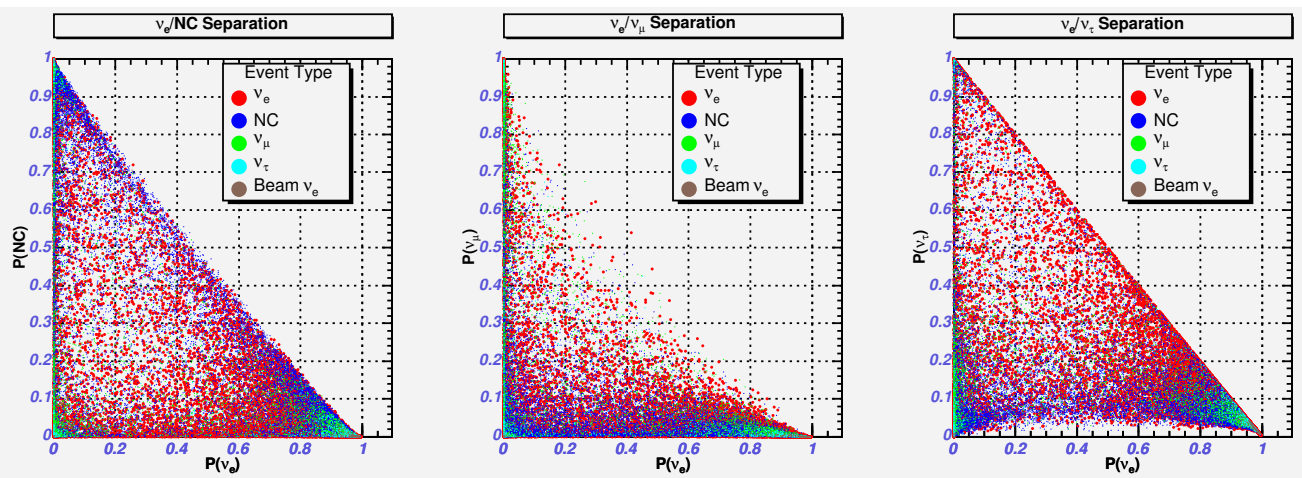

Figure 4.18: Event probability of belonging to the signal $\nu_{e}$ group plotted against the other probabilities. Although most of the true $\nu_{e}$ signal clusters in the lower bottom right corner, corresponding to high values of $\nu_{e}$ probability and low values of other probabilities, it is apparent that a large number of $\mathrm{NC}$ and $\mathrm{CC} \nu_{\mu}$ events follow the same trend. These events constitute the main source of background to the $\nu_{e}$ appearance measurement. These plots are not weighted by oscillation probability or exposure.
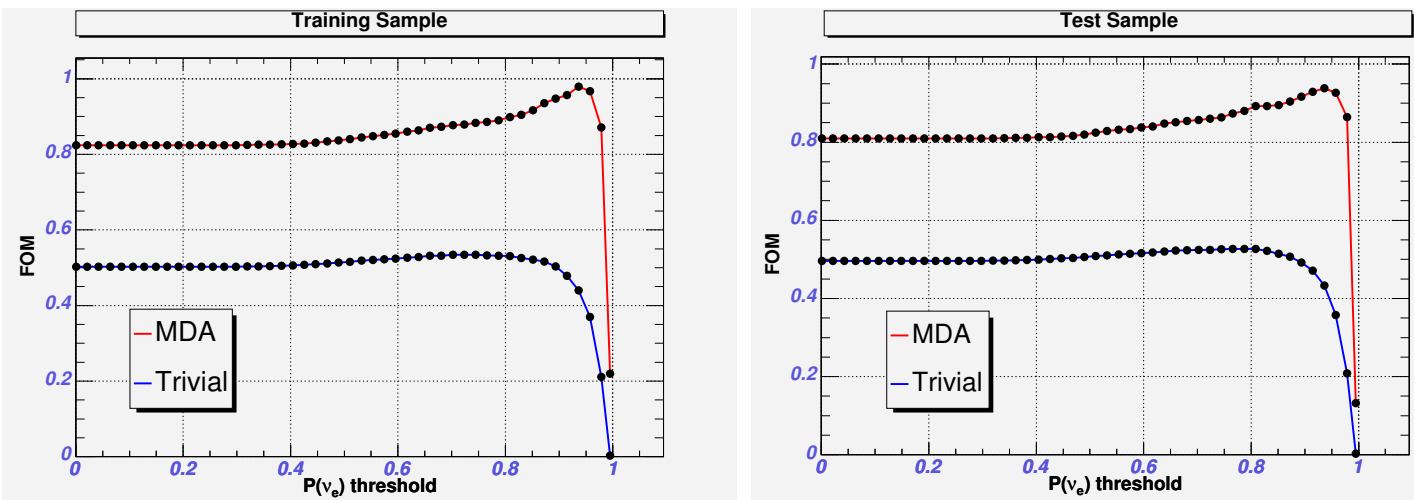

Figure 4.19: FOM vs. $\nu_{e}$ probability threshold. The left plot shows the FOM obtained for different values of the threshold cut obtained from the classification of events in the Training sample. The cut value (0.94, in this case) corresponding to the maximum FOM value is used for classification on the Test sample. The plot on the right shows the FOM evolution from classification in the Test sample. As can be seen, the choice of threshold cut from the Training sample is very consistent with the maximum obtainable $F O M$ value in the Test sample. A curve corresponding to a trivial classifier-consisting of choosing every event in the sample as signal $\nu_{e}$-is also displayed for comparison. 


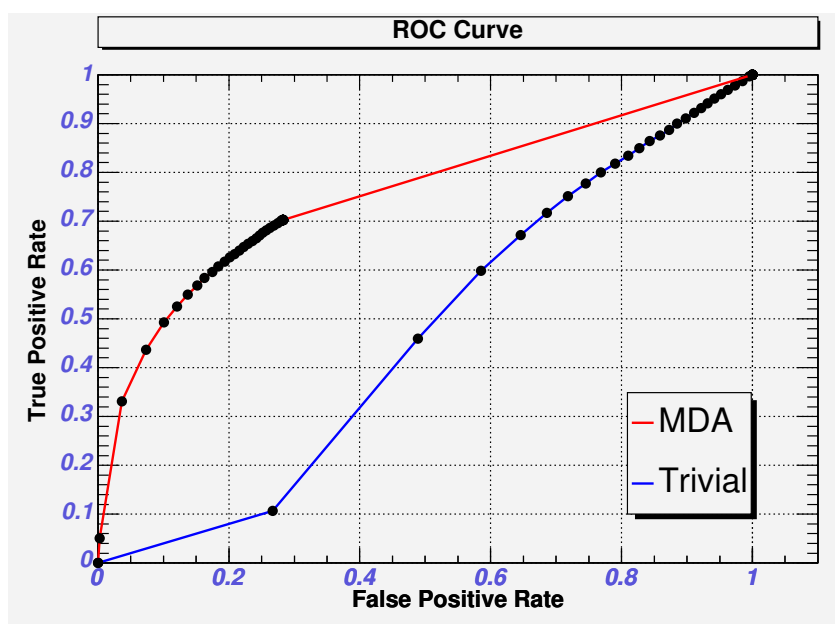

Figure 4.20: Receiver Operating Characteristic curve for the MDA method, compared with a trivial classifier. Each point in the curve represents the true positive and false positive rates calculated for a given threshold cut. The points with higher false positive rates correspond to lower values of the probability threshold cut.

\subsubsection{MDA Classification Results}

In this section, the classification results of different MDA runs on the Test sample are shown. In the tables that follow, the rows describing the sample content display the total event numbers for each event type. However, the rows related to MDA classification display only the number of events classified as the $\nu_{e}$ appearance signal.

Table 4.3 summarizes the values obtained weighted with SuperK oscillation parameters, a small value of $\theta_{13}$ corresponding to $\left|U_{e 3}\right|^{2}=0.01$, and a 2.5 year exposure.

With the purpose of deciding on the best method to use for classification on the Mock Data sample, it is appropriate to use our best knowledge of what oscillation parameters were used in the sample generation. This knowledge comes from the MINOS CC Analysis group, which determined the oscillation parameters in the Mock Data sample to be $\Delta m_{23}^{2}=2.175 \times 10^{-3} \mathrm{eV}^{2}$ and $\sin ^{2} 2 \theta_{23}=0.925$. Although the events classified by each method are the same irrespective of oscillation weights, the obtained FOM will not scale linearly when the oscillation weight varies. This is because each method selects different proportions of event types that, NC excepted, will scale differently with oscillation variations. The exposure weighting scales every 


\begin{tabular}{|c|c|c|c|c|c|c|c|}
\hline & Thresh. & $\mathrm{CC} \nu_{e}$ & $\mathrm{NC}$ & $\mathrm{CC} \nu_{\mu}$ & $\mathrm{CC} \nu_{\tau}$ & $\mathrm{Beam} \nu_{e}$ & $\mathrm{FOM}$ \\
\hline \hline Sample with cuts & - & 24730 & 22986 & 5666 & 1393 & 350 & - \\
with weights & - & 17.52 & 569.2 & 186.5 & 13.22 & 12.90 & 0.63 \\
\hline MDA method A & 0.94 & 6.57 & 33.35 & 9.61 & 2.10 & 4.58 & 0.93 \\
\hline MDA method B & 0.98 & 5.01 & 19.03 & 2.78 & 1.49 & 2.94 & 0.98 \\
\hline MDA method C & 0.99 & 5.10 & 16.48 & 2.61 & 1.33 & 3.50 & 1.04 \\
\hline MDA method D & 0.99 & 5.80 & 17.96 & 3.80 & 1.55 & 3.80 & 1.11 \\
$\Delta m_{23}^{2}=3.0 \times 10^{-3} \mathrm{eV}^{2}$ & 0.99 & 7.28 & 17.96 & 2.99 & 2.18 & 3.78 & 1.40 \\
\hline
\end{tabular}

Table 4.3: Comparison table showing the classification results obtained for four different MDA methods. The oscillation probability weight was calculated using the parameters $\Delta m_{23}^{2}=2.5 \times 10^{-3} \mathrm{eV}^{2}$ and $\sin ^{2} 2 \theta_{23}=1$, further assuming that $\left|U_{e 3}\right|^{2}=0.01$. The values presented correspond to an exposure of $9.25 \times 10^{20}$ POT. An additional weighted result was included for method $\mathrm{D}$, illustrating the potential improvement in the value of $F O M$ if $\Delta m_{23}^{2}=3.0 \times 10^{-3} \mathrm{eV}^{2}$.

event equally, so it is irrelevant in making the decision of which method to adopt. However, for completeness, the same exposure used in the Far Mock Data sample is used. The results obtained are summarized in Table 4.4 .

\begin{tabular}{|c|c|c|c|c|c|c|c|}
\hline & Thresh. & $\mathrm{CC} \nu_{e}$ & $\mathrm{NC}$ & $\mathrm{CC} \nu_{\mu}$ & $\mathrm{CC} \nu_{\tau}$ & Beam $\nu_{e}$ & $\mathrm{FOM}$ \\
\hline \hline Sample with cuts & - & 24730 & 22986 & 5666 & 1393 & 350 & - \\
with weights & - & 8.61 & 453.89 & 181.58 & 7.57 & 10.34 & 0.33 \\
\hline MDA method A & 0.94 & 3.10 & 26.70 & 9.21 & 1.19 & 3.67 & 0.48 \\
\hline MDA method B & 0.98 & 2.39 & 15.35 & 2.87 & 0.86 & 2.36 & 0.52 \\
\hline MDA method C & 0.99 & 2.41 & 13.24 & 2.58 & 0.76 & 2.81 & 0.55 \\
\hline MDA method D & 0.99 & 2.75 & 15.19 & 3.81 & 0.89 & 3.05 & 0.58 \\
\hline
\end{tabular}

Table 4.4: Comparison table showing the classification results obtained for four different MDA methods. The oscillation probability weight was calculated using the parameters $\Delta m_{23}^{2}=2.175 \times 10^{-3} \mathrm{eV}^{2}$ and $\sin ^{2} 2 \theta_{23}=0.925$, further assuming that $\left|U_{e 3}\right|^{2}=0.01$. The values presented correspond to an exposure of $7.4 \times 10^{20} \mathrm{POT}$.

For the parameters used, Method D yields the best results, as measured by the FOM criterion. This method focuses on the distinction between QE CC $\nu_{e}$ and $\mathrm{NC}$ events, using only these two groups to classify the sample. This method will thus be used for classification of the Near and Far Mock Data samples to be described in the next chapter. Fig. 4.21 and Fig. 4.22 show the stepwise selection output of the method and its FOM evolution with the threshold cut, respectively. 


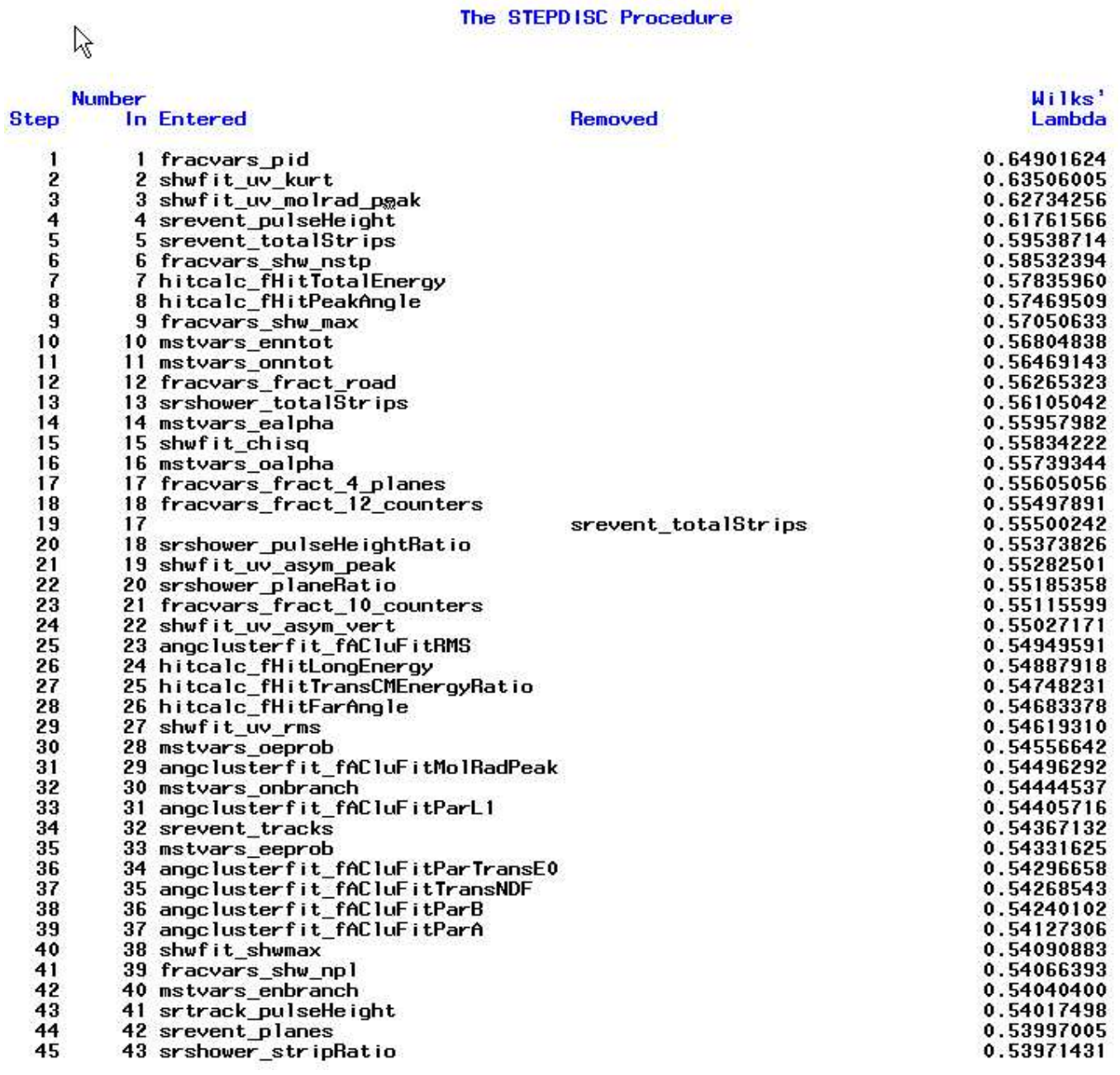

Figure 4.21: Stepwise variable selection for MDA method D. The output lists the input variables in descending discriminant power, as measured by the Wilks' $\Lambda$ criterion. Only the first 45 steps are shown. The 19 best variables were used to assemble the discriminant function.

\subsubsection{MDA Performance}

Having decided on an MDA method to apply in the final steps of the analysis, we now look in some detail at the method performance.

\section{Efficiency and Purity}

Common performance measures are obtained using the efficiency and purity concepts. Efficiency measures the quality of signal identification By comparing the number of 


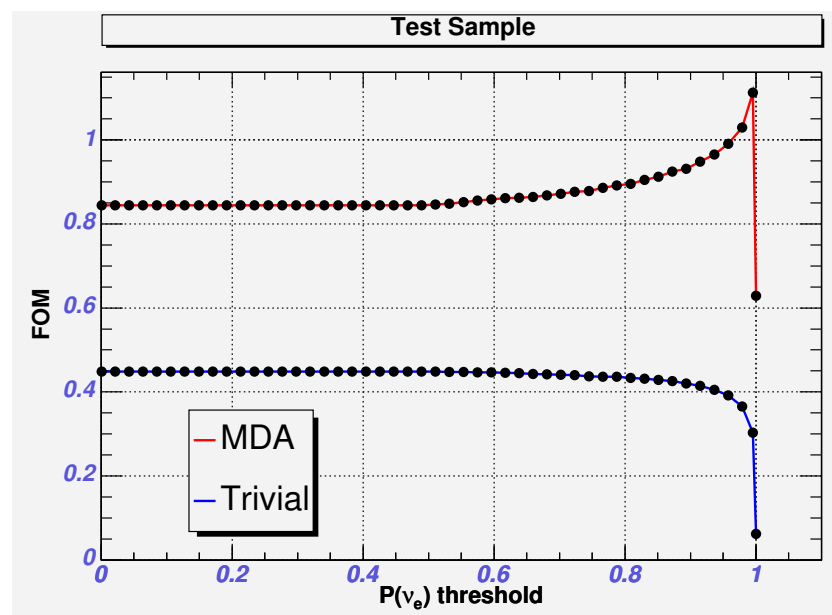

Figure 4.22: FOM vs. $\nu_{e}$ probability threshold for the determined best MDA classifier. The maximum FOM value corresponds to a threshold cut value of 0.99 . The same plot for a trivial classifier is also shown.

correctly identified signal events with the true total number contained in the sample:

$$
\text { Efficiency }=\frac{T P}{T P+T N}
$$

where $T P$ is the number of True Positives identified by the method and $T N$ the number of True Negatives, corresponding to misidentifed signal. Purity measures the extent of the contamination of the selected signal by background and is given by:

$$
\text { Purity }=\frac{T P}{T P+F P}
$$

where $T P$ is the number of True Positives as before and $F P$ is the number of False Positives.

The efficiency-purity variation with probability threshold is shown in Fig. 4.23. For some applications that make use of artificial neural networks, the point of crossing of the two curves constitutes a good approximation to the ideal choice of the method's output cut-off. It is interesting to notice that for our MDA application, as is apparent in the figure, the crossing point is consistent with the choice of threshold determined from FOM measurements.

It is also interesting to understand how the method efficiency varies with the reconstructed event energy. This is illustrated in Fig. 4.24. In the visible energy 


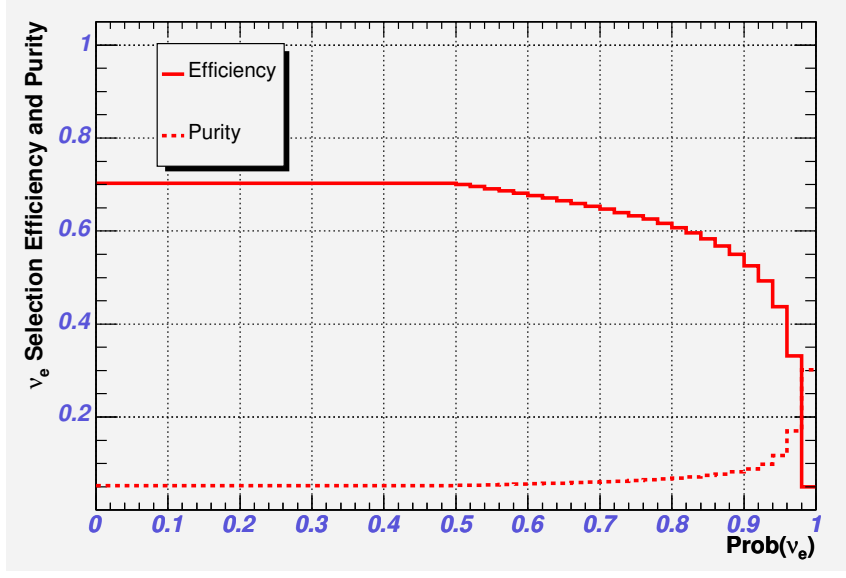

Figure 4.23: MDA efficiency and purity as a function of the probability threshold cut. The crossing of the two curves occurs at a cut value consistent with the chosen threshold of 0.99 for this method.

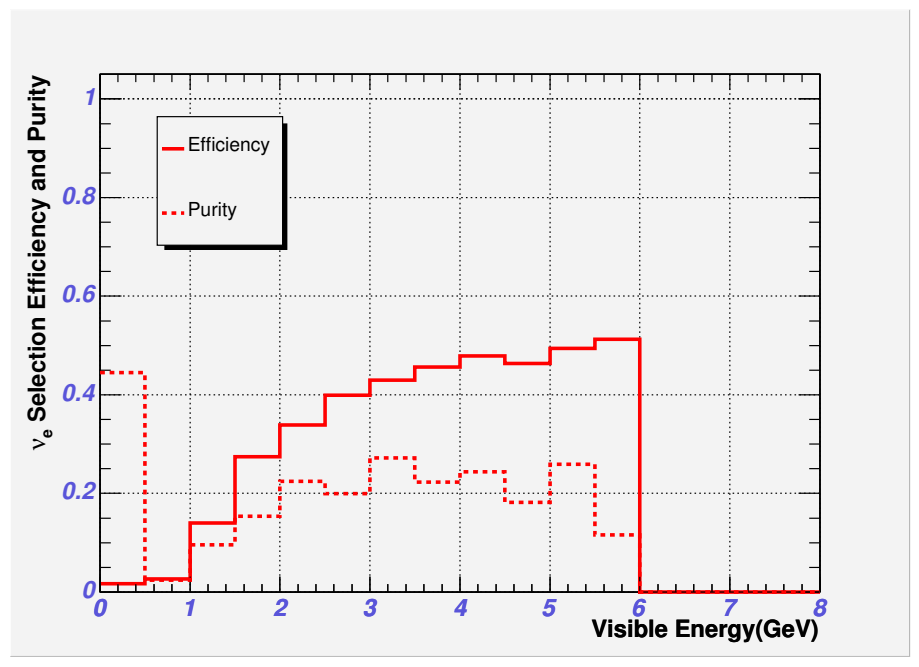

Figure 4.24: MDA efficiency and purity as a function of the reconstructed event energy. The large purity value in the first bin is due to low statistics and is not significant. The MDA method performs better in the range of 3 to $5 \mathrm{GeV}$. The curves stop at $6 \mathrm{GeV}$ due to the visible energy cut applied prior to MDA classification.

range of 3 to $5 \mathrm{GeV}$, the sample purity has the highest values, higher than $20 \%$ and the efficiency remains around $50 \%$. After $5.5 \mathrm{GeV}$, the sample contamination increases. For the oscillation parameters discussed in this thesis, the energy range of 
2-5 GeV corresponds to where most of the hypothetical $\nu_{e}$ appearance signal will be found, thus it is reassuring to see that the MDA method works best in most of that visible energy range.

\section{Energy Distributions}

The visible energy distribution of the classified events is displayed in Fig. 4.25 in SigCor units. The selected beam $\nu_{e}$ events tend to peak at larger values of visible energy. Some non-negligible amount of NC events show very low amounts of visible energy and should be investigated further by visual scanning.

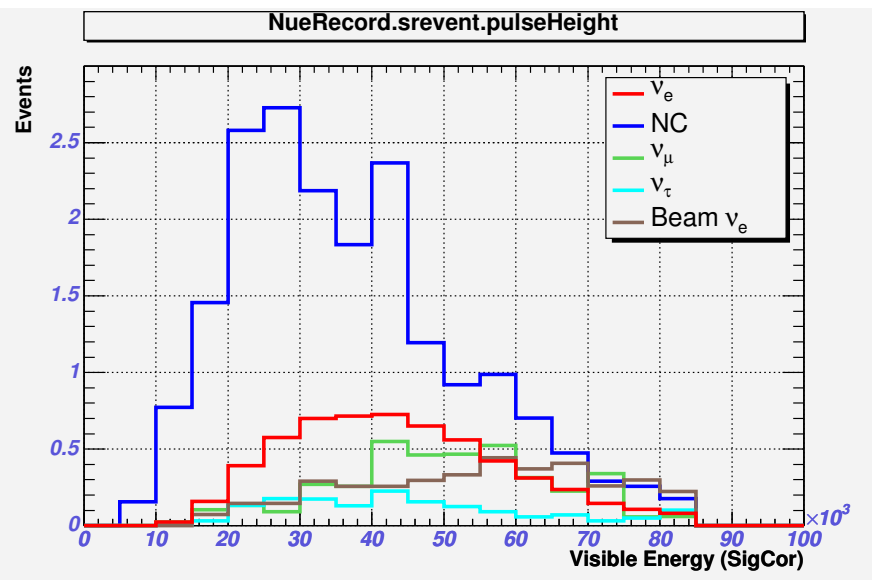

Figure 4.25: Visible energy distribution of classified events (1 GeV $\sim 15000$ SigCor).

To form a better idea of where the potential $\nu_{e}$ appearance signal, as selected by MDA, should be found, we plot the signal events as an excess over the expected background in Fig. 4.26. As can be noticed in the figure, the energy distribution of the background follows closely the signal distribution. It is therefore particularly difficult to claim a signal discovery in this situation, as the excess signal could correspond to a mere background fluctuation.

\section{Truth Quantities}

To further assess the performance of the classification method, we may look at distributions of the truth variables, which are available in the Test sample. In Fig. 4.27, 


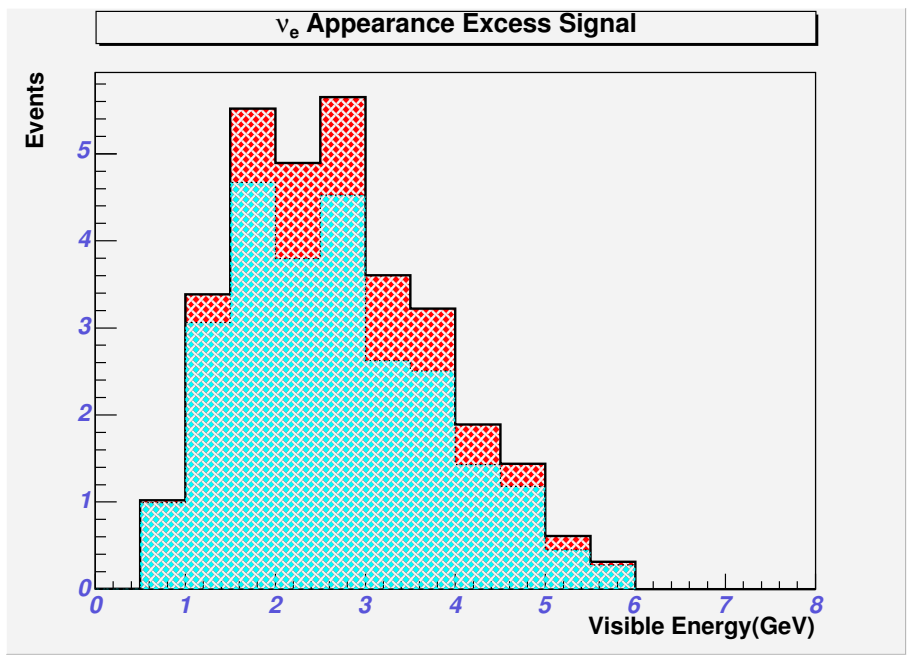

Figure 4.26: Visible energy distribution of the excess signal $\nu_{e}$ appearance over the expected background. The summed background histogram is displayed in cyan and the excess $\nu_{e}$ signal in red.

for all the events classified as signal $\nu_{e}$, the numbers of quasi-elastic, resonance production, deep inelastic scattering and coherent pion production, divided into event types is shown.

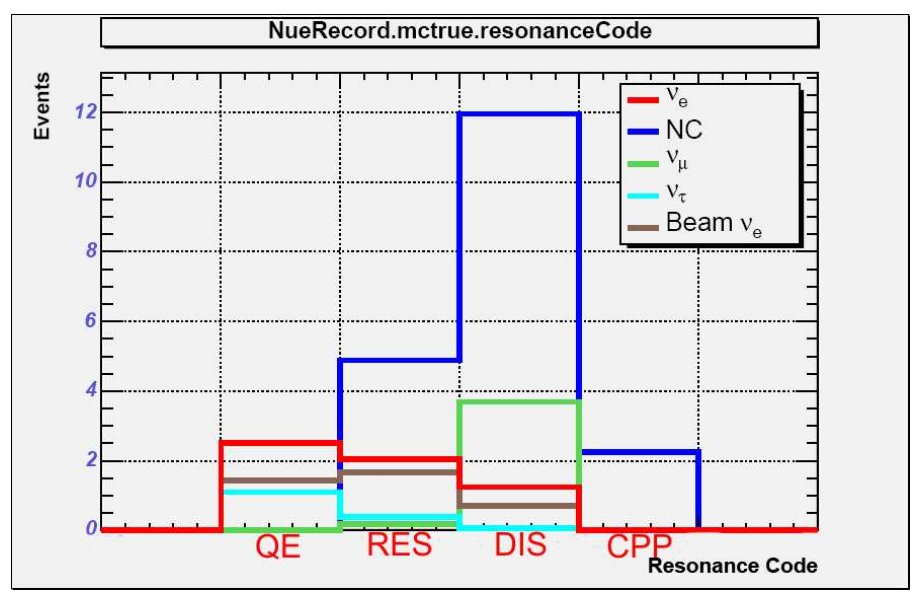

Figure 4.27: Distribution of true resonance code for MDA classified events, showing the number of selected events from each type for quasi-elastic, resonance production, deep inelastic scattering and coherent pion production interactions.

In Fig. 4.28, the distribution of electromagnetic shower energy fraction is illus- 
trated, alongside with the $y$ inelasticity distribution. In Fig. 4.29, the incoming neutrino energy distribution is plotted together with the event length measured in number of planes. This last quantity is not a truth quantity, but it does provide useful information for a discussion of the results observed in the other plots.
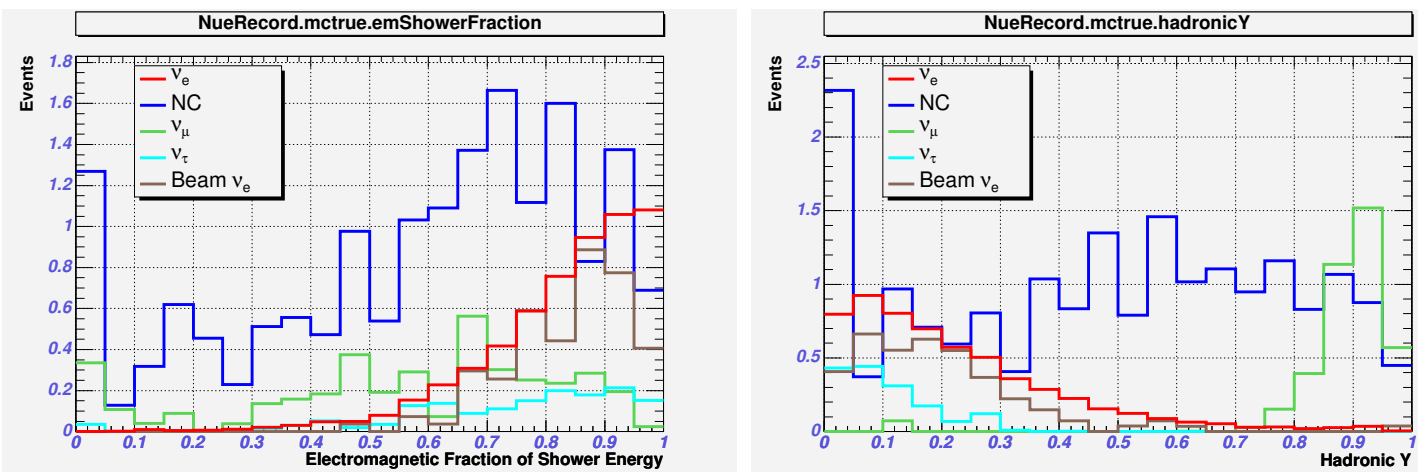

Figure 4.28: The left plot displays the EM shower energy fraction distribution of MDA classified events, whereas the plot on the right shows the $y$ distribution. Most selected NC events have high EM fraction values and about all of the selected CC $\nu_{\mu}$ events display high inelasticity.
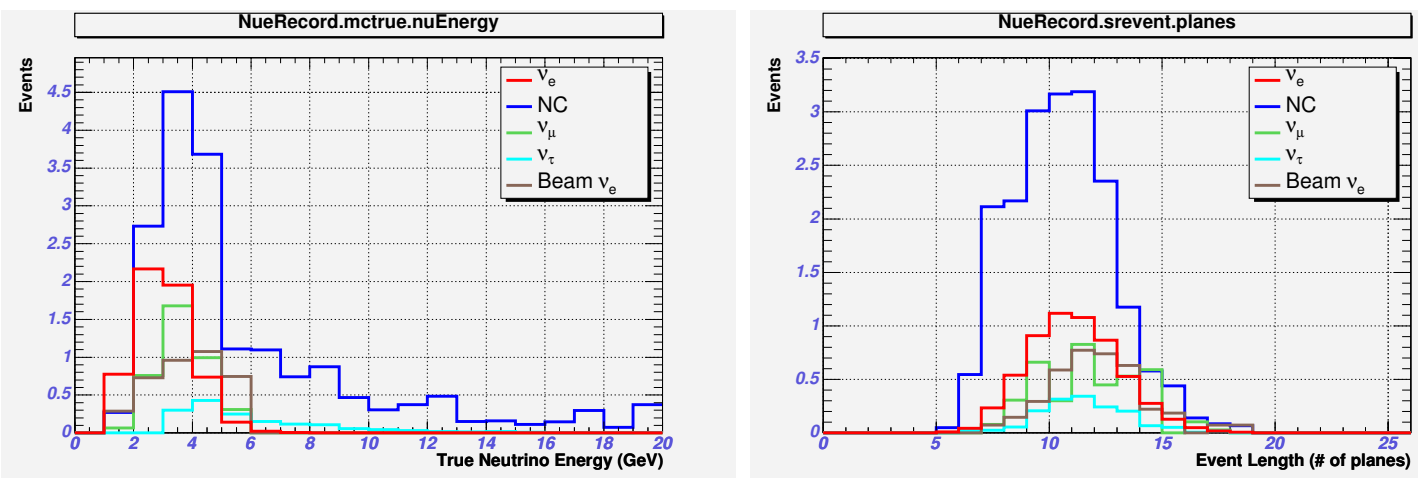

Figure 4.29: The left plot illustrates the distribution of true incoming neutrino energy for MDA classified events, whereas the plot on the right shows the event length in terms of number of planes. It can be seen that the selected beam $\nu_{e}$ originate from the same energy range as the signal. As expected, the selected $\nu_{\mu}$ events tend to be very short.

From the analysis of the truth plots, several considerations come to mind: 
i) Most of the selected signal $\nu_{e}$ originates from QE interactions, consistent with the MDA method applied.

ii) A majority of the NC background events display a large electromagnetic energy fraction, but the amount of low electromagnetic energy fraction events is not negligible. Furthermore, many events result from relatively low energy incoming neutrinos.

iii) All of the CC $\nu_{\mu}$ background is due to short events with very high $y$. However, many events have medium or low values of electromagnetic energy fraction, a fact which, in conjunction with the previous point, seems to indicate the MDA method confuses hadronic with electromagnetic showers to some degree. It is likely the short muon track low pulse height strips within the hadronic shower help to simulate an electromagnetic shower core.

iv) The selected CC $\nu_{\tau}$ background follows the signal $\nu_{e}$ behavior very closely, indicating that in the majority of the selected background events the $\tau$ decayed into an electron or into an hadronic system where the $\pi^{0}$ carried most of the momentum.

v) The beam-induced $\nu_{e}$ background logically presents the larger feature overlap with the $\nu_{e}$ appearance signal, but the use of Near Detector data will constrain the extent of its contamination.

To attempt to understand in more detail the remarks in points ii) and iii), visual scanning of individual events may be useful. A few events illustrating those points are depicted in Fig. 4.30, Fig. 4.31 and Fig. 4.32.

A limited visual scanning of events such as the ones exemplified in these plots indicates that relatively low energy NC background events will most likely have a very large electromagnetic shower fraction. In addition, it suggests that events with high multiplicity in the hadronic final state will display hadronic showers similar enough to electromagnetic showers, confusing the classification method. It is possible that this separation can be improved. The consistency of the MDA results with other 


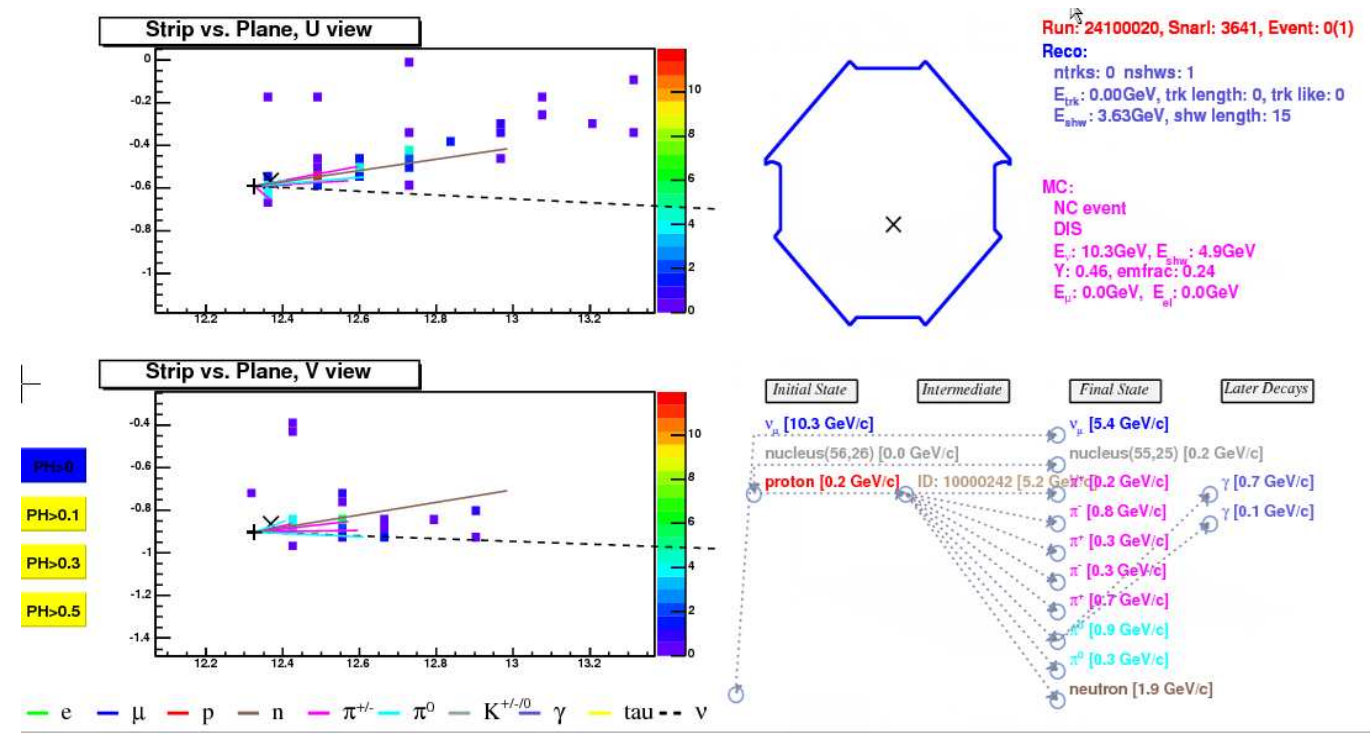

Figure 4.30: Example of a selected NC background event displaying a low value of electromagnetic shower energy fraction. It can be seen that the shower shape is almost entirely due to the secondary $\pi^{ \pm}$.
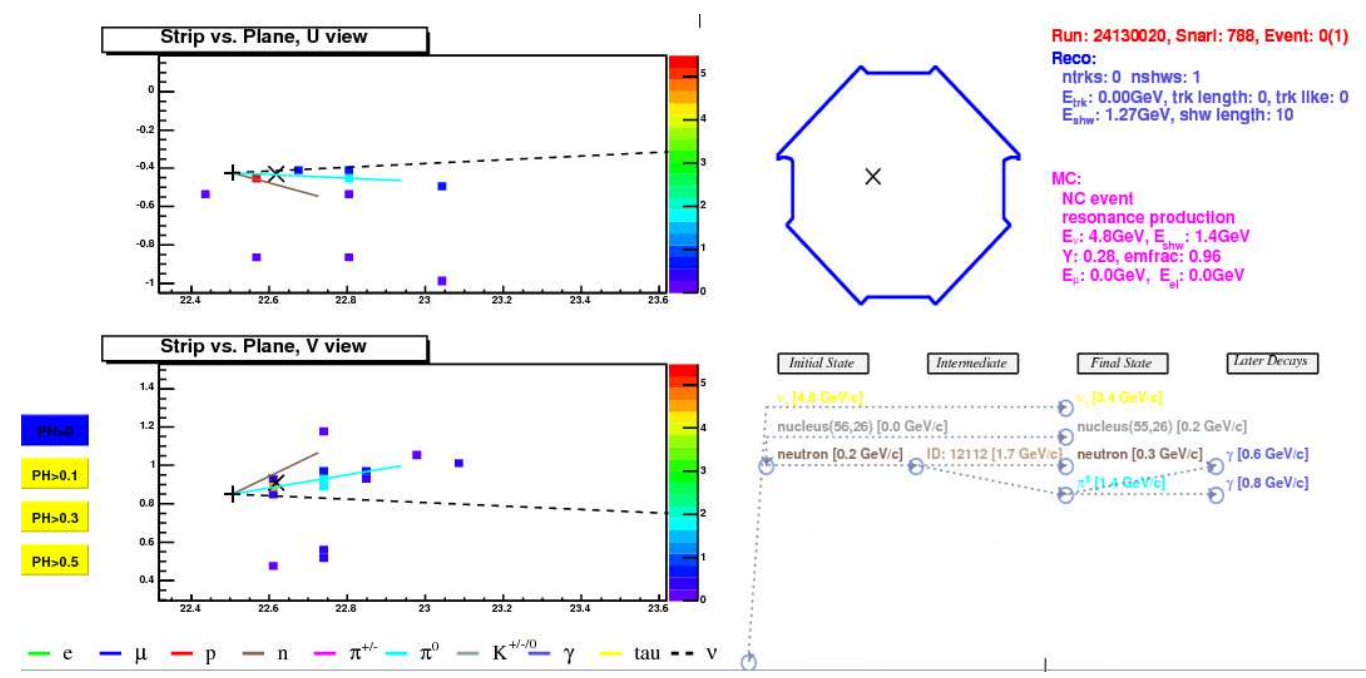

Figure 4.31: Example of a NC background event that displays a low amount of visible energy. The incoming neutrino is moderately energetic, but the total amount of energy deposited is low. However the electromagnetic energy fraction is very high, leading to the misclassification.

analyses indicates the limitation is not in the method, but rather in the amount of 


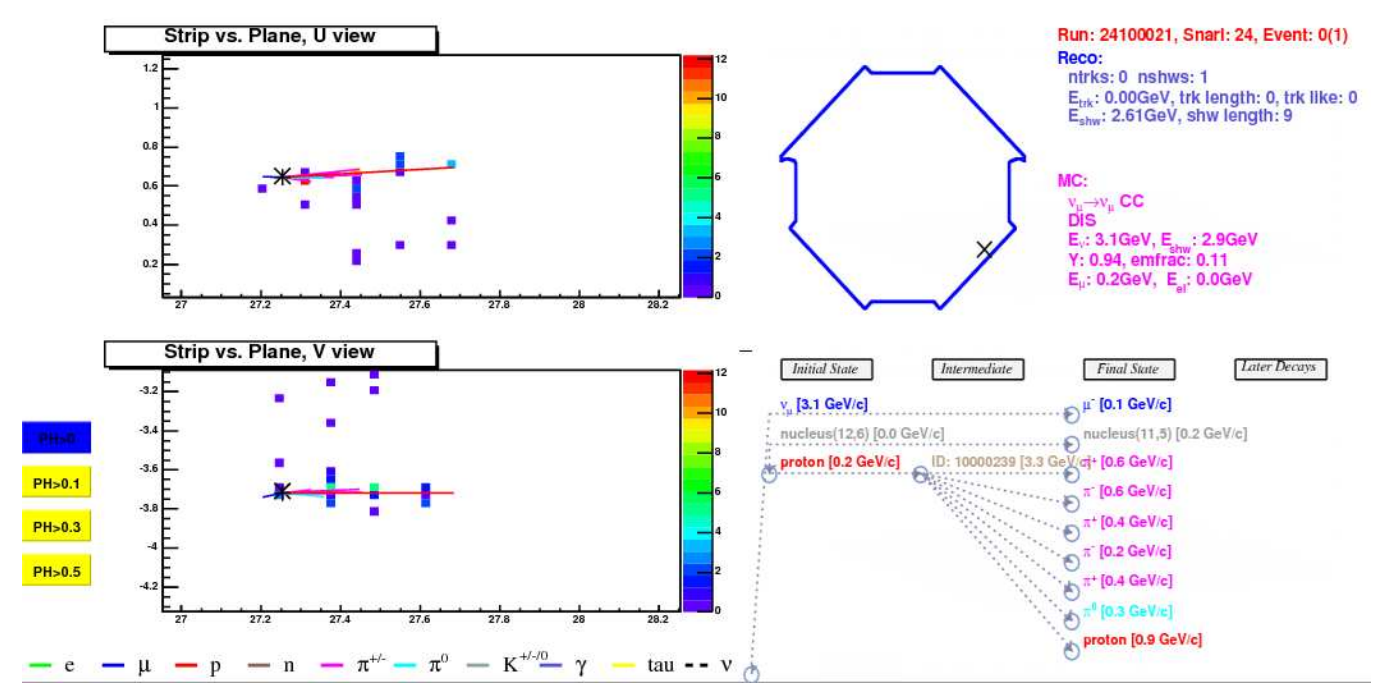

Figure 4.32: Example of a $\mathrm{CC} \nu_{\mu}$ background event with low electromagnetic energy fraction. In this event, with a very large value of $y$, the shower is almost exclusively due to hadronic energy deposition by the $\pi^{ \pm}$system, in similar fashion to the event depicted in Fig.4.30.

information available for classification. A software package that identifies subshower components within a showering event is presently being readied for production and could provide that additional information. An extensive visual scanning effort dedicated to $\nu_{e}$ appearance identification is currently underway at Tufts and the pattern recognition algorithms developed could further the classification capabilities of the automated methods.

In the next chapter, the described MDA method will be applied to the Near and Mock Data samples, in order to fulfill the Mock Data Challenge. 


\section{Chapter 5}

\section{Results and Discussion}

In this Chapter, the multivariate discriminant method investigated in Chapter 4 that yielded the best results is applied to classification of the Near Monte Carlo and Far and Near Mock Data samples. A discussion of the NEUGEN systematic crosssection parameters varied in the Mock Data Challenge fits is presented along with a description of the reweighting mechanism used to perform those fits and understand the method's sensitivity to possible sets of oscillation parameters. The Monte Carlo samples are fitted to their Mock Data counterparts to evaluate the existence of a $\nu_{e}$ appearance signal in the MDC. Finally, the true $\nu_{e}$ oscillation parameters used in the MDC are revealed and a discussion of the results obtained is presented. The sensitivity and fit results presented in the Chapter were obtained through the use of tools available in the NueAna framework that were primarily written by P. Vahle and C. Smith. Ref. [98] contains practical information on the usage of these tools.

\subsection{Classification of the Near Sample}

The classified Near MC sample will be used to fit the Near Mock Data spectrum, applying the added statistics to further constrain the NEUGEN parameters used in the Challenge set.

In order to perform MDA classification on the Near Detector MC sample, the cuts described in the previous Chapter are applied. The classification results obtained by running the MDA method D on the Near MC sample are shown in Table 5.1.

It should be noted that the events selected from the Near samples correspond 


\begin{tabular}{|c|c|c|c|c|}
\hline & Thresh. & NC & CC $\nu_{\mu}$ & Beam $\nu_{e}$ \\
\hline \hline Sample totals & - & 101200 & 641247 & 8937 \\
Sample with cuts & - & 21728 & 18965 & 888 \\
\hline MDA method D & 0.99 & 822 & 370 & 306 \\
\hline
\end{tabular}

Table 5.1: Number of events in the Near MC sample separated by type. The total numbers in the sample and the numbers after cuts are shown. The last row displays the number of events classified as $\mathrm{CC} \nu_{e}$ for each event class.

solely to background to the signal being measured, which can be extrapolated to the Far Detector, as obviously no $\nu_{\mu} \rightarrow \nu_{e}$ oscillation signal is expected to be observed at the Near Detector.

\subsection{NEUGEN Systematics and MC Reweighting}

As mentioned in Chapter 3, undisclosed values for some of the neutrino cross-section parameters used in NEUGEN were employed in the Mock Data sample generation. These parameters were the axial mass for quasi-elastic interactions QEL_MA, the axial mass for single pion production interactions RES_MA and the deep inelastic scattering "fudge" factor, dubbed KNO.

The QEL_MA and RES_MA parameters arise in the theoretical modeling of neutrinohadron interactions at low energy, in which the neutrino scatters off an entire nucleon rather than the constituent partons. As seen in Ref. [99], the general hadronic current for these processes can be expressed as a function of the form factors of the nucleon, namely the scalar $F_{S}$, pseudo-scalar $F_{P}$, vector $F_{V}$, axial-vector $F_{A}$, and tensor $F_{T}$ form factors. To first order, $F_{S}$ and $F_{T}$ can be set to 0 . The $F_{P}$ inclusion leads to small corrections to the quasi-elastic cross-sections. The squared momentum transfer $\left(q^{2}\right)$ dependence of $F_{V}$ can be written as the dipole form $F_{V} \sim\left(1-\frac{q^{2}}{M_{V}^{2}}\right)^{-2}$ and the value of the vector mass $M_{V}$ is determined with high accuracy through electron scattering experiments to be $M_{V}=0.71 \mathrm{GeV} / \mathrm{c}^{2}$. However, for the hadronic axial current $F_{A}$, the $q^{2}$ dependence has to be inferred or measured. In analogy with the 
vector case it is common to assume the same dipole form:

$$
F_{A}=\frac{-1.23}{\left(1-\frac{q^{2}}{M_{A}^{2}}\right)^{2}},
$$

where the numerical value in the numerator is obtained from $\beta$-decay measurements. This phenomenological expression agrees well with cross-section measurements carried out in low energy neutrino scattering experiments. The value of the axial-vector mass $M_{A}$ is extracted from the analysis of neutrino-nucleon scattering data and the global average value is found to be $M_{A}=1.032 \pm 0.36 \mathrm{GeV} / c^{2}$. This value is set as the default for the QEL_MA and RES_MA parameters used by NEUGEN.

The third parameter, KNO, is found in the approach used by NEUGEN to compute the total cross-section at a fixed energy. This approach assumes:

$$
\sigma_{\text {total }}=\sigma_{q e l}+\sum_{j}\left(\sigma_{r e s}^{j}+f_{j} \sigma_{d i s}^{j}\right)
$$

where the $j$ refer to the final state multiplicity. The $f_{j}$ factors are especially relevant for processes with low multiplicity in the final state and are determined from comparisons with data for each exclusive final state at a fixed energy. NEUGEN models the hadronization of the final state produced through DIS using the Koba, Nielsen, Olesen (KNO) approach [100]. This method can be interpreted as a mechanism of balancing the different contributions to deep inelastic scattering processes (in which the neutrino scatters off the partons inside the nucleon) with low multiplicity in the final states. One of the main contributions results from considering that the partons in the nucleon behave as free particles, which agrees well with the data for high energies, and the other from assuming the parton system is bound by a relativistic harmonic potential, the so called Feynman-Kislinger-Ravndal model (FKR) [101]. The well known Rein-Sehgal model [102] employs the FKR characterization of baryon resonances to successfully describe resonance production processes.

The unknown KNO scaling factor used in the Mock Data generation corresponds to $f_{2}$ and thus affects the calculation of the cross-sections for DIS interactions with two particles in the hadronic final state.

In the reweighting mechanism described below and used to obtain the results presented in this Chapter, the values of the three NEUGEN parameters described are 
not used directly. A relative weight is employed instead, so that, for instance, the default value of QEL_MA would be used if a weight of 1.0 is passed to the reweighting code, a value $4 \%$ higher than the default would be obtained with a weight of 1.04 and so on. The default values of the parameters in NEUGEN and their nominal weights in the reweighting code are summarized in Table 5.2.

\begin{tabular}{|c|c|c|}
\hline NEUGEN Parameter & NEUGEN Default Value & Nominal Weight \\
\hline \hline QEL_MA & $1.032 \pm 4 \%\left(\mathrm{GeV} / \mathrm{c}^{2}\right)$ & 1.0 \\
\hline RES_MA & $1.032 \pm 4 \%\left(\mathrm{GeV} / \mathrm{c}^{2}\right)$ & 1.0 \\
\hline KNO & $1.0 \pm 3 \%$ & 1.0 \\
\hline
\end{tabular}

Table 5.2: Default values and nominal weights for the NEUGEN parameters used in the sensitivity and MDC fits.

These parameters will be part of the set of numbers allowed to vary in the MDC fit. To perform the fit or calculate sensitivities, it is necessary to reweight each MDA-selected event contained in the Near and Far MC samples with different sets of values of fitting parameters. Using tools available in the NueAna framework, a parameter value grid is defined and the many instances obtained for each event are stored in a Reweight ntuple. The reweighting of the events is achieved by using NEUGEN and the event truth information describing the incoming neutrino energy, flavor $\left(\nu_{\mu}, \nu_{e}\right.$ or $\left.\nu_{\tau}\right)$ and interaction type (CC quasi-elastic or NC elastic, $\mathrm{CC}$ or $\mathrm{NC}$ resonance production, $\mathrm{CC}$ or $\mathrm{NC}$ deep inelastic scattering, $\mathrm{CC}$ or $\mathrm{NC}$ coherent pion production). The truth information and a set of cross-section parameters is passed to NEUGEN, which recalculates the interaction parameters and returns a weight for the event. If a set of oscillation parameters is provided, the reweighting procedure will also recalculate the oscillation probability value for the event. This reweighting method is very time consuming, thus limiting the size of the grid of values that can be used.

\subsection{MINOS Sensitivity to $\nu_{e}$ Appearance}

To estimate the range of oscillation parameter values that can be excluded using the presented MDA method, a comparison with a null $\nu_{\mu} \rightarrow \nu_{e}$ oscillation solution is 
performed. The NEUGEN weights are kept at the nominal values, the value of the "atmospheric" mixing angle is fixed at the MDC CC group best fit, $\sin ^{2}\left(2 \theta_{23}\right)=0.925$, and a grid of values for $\Delta m_{23}^{2}$ and $\sin ^{2}\left(2 \theta_{13}\right)$ is used. A histogram of the energy spectrum of selected events in the Far sample is weighted using different values for the parameter $\Delta m_{23}^{2}$, while $\sin ^{2}\left(2 \theta_{13}\right)=0$. Each one of these histograms, corresponding to a fixed value of $\Delta m_{23}^{2}$, is fitted to the matching histogram in which $\sin ^{2}\left(2 \theta_{13}\right)$ is allowed to vary. Each of these fits corresponds to a $1 \mathrm{D}$ fit in $\sin ^{2}\left(2 \theta_{13}\right)$, but can be expressed in a 2D plot. All histograms are normalized with the two values of proton intensity. The $\chi^{2}$ function used to fit the histograms was obtained from Section 28.3.4 of Ref. [103]:

$$
\chi^{2}\left[\sin ^{2}\left(2 \theta_{13}\right)\right]=2 \sum_{i=1}^{b i n s}\left[\left(N_{i}^{t h}-N_{i}^{o b s}\right)+N_{i}^{o b s} \ln \left(N_{i}^{o b s} / N_{i}^{t h}\right)\right]
$$

where, for our purposes, $N_{i}^{\text {th }}$ denotes the $i$-th bin of the null oscillation histogram and $N_{i}^{o b s}$ its oscillation solution counterpart. In bins where $N_{i}^{o b s}=0$, the second term is zero.

Since we are fitting a single parameter, $\sin ^{2}\left(2 \theta_{13}\right)$, a $90 \%$ exclusion region is defined by the set of points with $\Delta \chi^{2} \leqslant 2.71$ [104], where $\Delta \chi^{2}=\chi_{i}^{2}-\chi_{\min }^{2}$.

The results obtained for $7.4 \times 10^{20}$ POT and $22.2 \times 10^{20}$ POT are displayed in Fig 5.1. A cursory glance at the figure indicates that in $\sim 2$ years of running, MINOS should be able to exclude a larger oscillation parameter region than the CHOOZ experiment, over the $\Delta m_{23}^{2}$ range favored by current atmospheric neutrino experiments, and therefore improve on the CHOOZ limit on $\nu_{\mu} \rightarrow \nu_{e}$ oscillations. For instance, if one takes SuperK's best fit value of $\Delta m_{23}^{2}$, at $\sin ^{2}\left(2 \theta_{23}\right)=0.925$, MINOS has the potential to probe $\sin ^{2}\left(2 \theta_{13}\right)<0.09$ in 2 years, which corresponds to a factor of $\sim 1.8$ improvement on the CHOOZ limit.

\subsection{Mock Data Fitting Results}

In this section, the MC Far and Near samples are fitted to their Mock Data equivalents in an attempt to identify the presence or absence of a $\nu_{\mu} \rightarrow \nu_{e}$ oscillation signal in the Mock Data. Before the fit can be carried out, the same set of cuts used for 

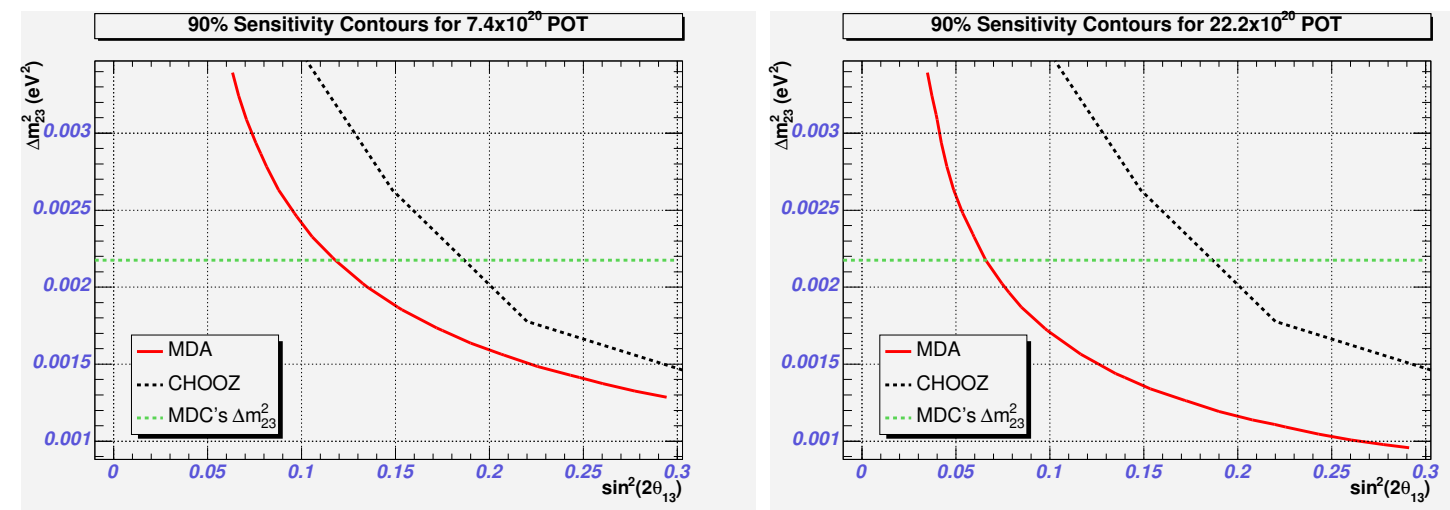

Figure 5.1: MDA sensitivity to $\nu_{\mu} \rightarrow \nu_{e}$ appearance for $7.4 \times 10^{20}$ POT (left) and $22.2 \times 10^{20}$ POT (right). The $90 \%$ limit from the CHOOZ experiment is shown for comparison. In both cases, the excluded parameter region is to the right and above of the contour lines. The MDC $\Delta m_{23}^{2}$ best fit from the MINOS CC analysis group is highlighted in green.

the MC samples is applied to the Mock Data ones and the MDA classification is performed in both the Far and Near Mock Data samples. The totals obtained are summarized in Table 5.3. Using the classified samples, two histograms divided in

\begin{tabular}{|c|c|c|}
\hline & Thresh. & Events \\
\hline \hline FD Mock Data (2 years) & - & 4174 \\
With cuts & - & 690 \\
MDA selected & 0.99 & 30 \\
\hline FD Mock Data (6 years) & - & 12644 \\
With cuts & - & 2057 \\
MDA selected & 0.99 & 106 \\
\hline ND Mock Data & - & 849608 \\
With cuts & - & 46312 \\
MDA selected & 0.99 & 1764 \\
\hline
\end{tabular}

Table 5.3: Number of events MDA classified as signal in the Near and Far Mock Data samples. The total numbers in the samples and the numbers after cuts are also shown.

20 1-GeV reconstructed energy bins are assembled for the Far and Near MC sets. An identical procedure is applied to the Far and Near Challenge (Mock Data) sets. In the case of the MC sets, a reweighted version of the Far and Near histograms is constructed for each set of fit parameter values. The MC reweighted histograms are 
then fitted to their Mock Data counterparts using a $\chi^{2}$ function analogous to the one described in Eq. 5.1, with $N^{\text {th }}$ corresponding to the MC Far and Near MC histograms and $N^{\text {obs }}$ to the respective Mock Data counterparts.

The parameters allowed to vary in the fits consist of the three NEUGEN crosssection parameters and the oscillation parameters $\Delta m_{23}^{2}, \sin ^{2}\left(2 \theta_{23}\right)$ varied by $1 \sigma$ with respect to the nominal values. $\sin ^{2}\left(2 \theta_{13}\right)$ is allowed to vary on a wider range. A parameter grid of $3 \times 3 \times 3 \times 3 \times 3 \times 21$ was used. For illustrative purposes, fits are presented where some or all of the parameters except $\sin ^{2}\left(2 \theta_{13}\right)$, which is the quantity we are interested in measuring, are kept at a nominal value. The nominal weights for the NEUGEN parameters and nominal values for the atmospheric oscillation parameters are described in Table 5.4. The range of fitted values for each parameter can be

\begin{tabular}{|c|c|}
\hline Fit Parameter & Nominal \\
\hline \hline QEL_MA & 1.0 \\
\hline RES_MA & 1.0 \\
\hline KNO & 1.0 \\
\hline$\Delta m_{23}^{2}\left(\mathrm{eV}^{2}\right)$ & $2.175 \times 10^{-3}$ \\
\hline $\sin ^{2}\left(2 \theta_{23}\right)$ & 0.925 \\
\hline
\end{tabular}

Table 5.4: Nominal weights and values for the fit parameters used in the Mock Data Challenge fits.

read from the example fit displayed on Figure 5.2.

Four types of fits were carried out in order to try to understand how the systematic effects of the cross-section parameter variation affect the final results:

i) Far Detector only, all parameters at nominal values, except for $\sin ^{2}\left(2 \theta_{13}\right)$.

ii) Far Detector only, all parameters allowed to vary.

iii) Far Detector + Near Detector, all parameters at nominal values, except for $\sin ^{2}\left(2 \theta_{13}\right)$.

iv) Far Detector + Near Detector, all parameters allowed to vary.

In the case of the Far+Near fits, the best fit values result from the combined fit of Far and Near. Two complete sets of these fits were run for intensities of $7.4 \times 10^{20}$ POT 

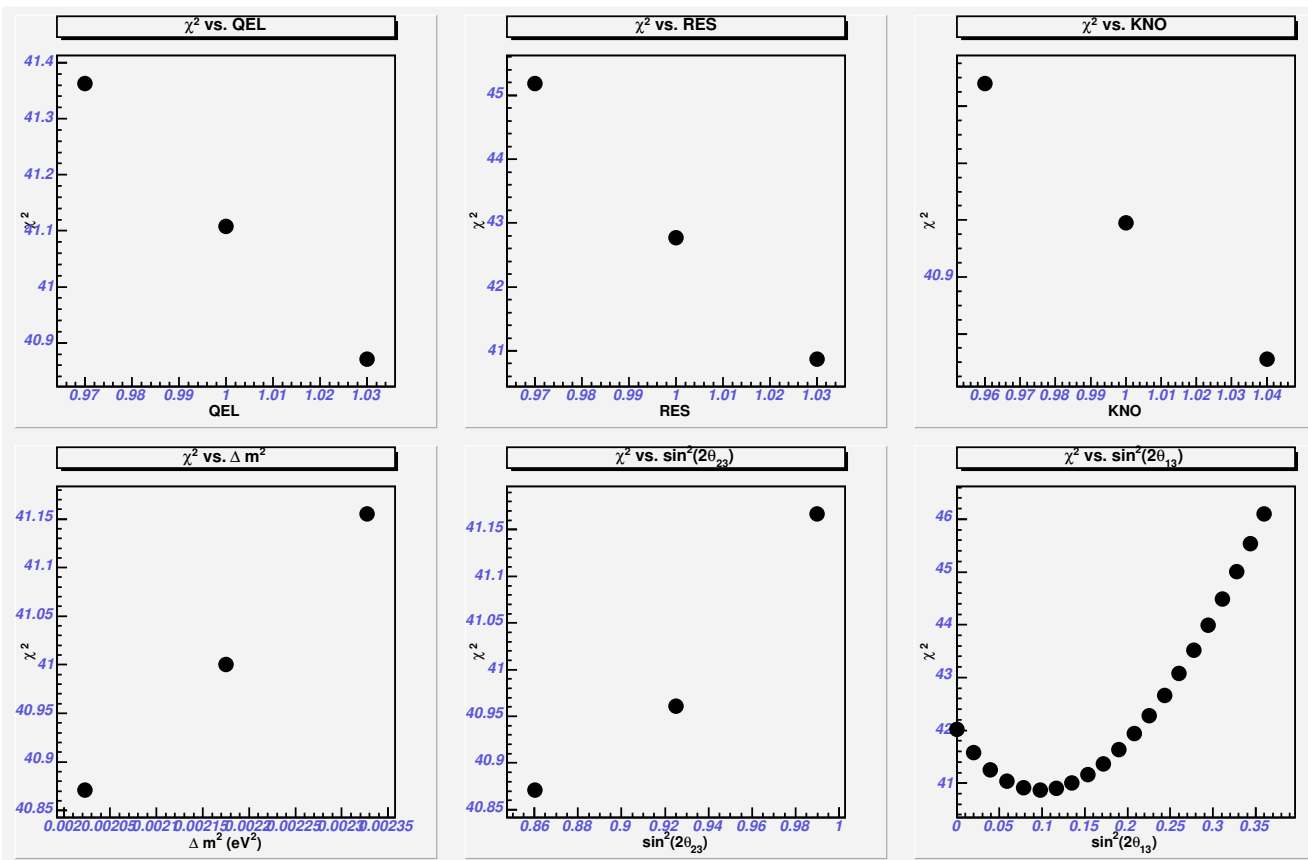

Figure 5.2: Evolution of $\chi^{2}$ fit value with the variation of Mock Data fit parameters. The possible values for the NEUGEN parameters used in the fits are shown in the top plots, whereas the bottom plots show the values used for the neutrino oscillation parameters. The evolution of the $\chi^{2}$ value for each parameter is calculated with all the other parameters held at their best fit value.

and $22.2 \times 10^{20}$ POT. The former gives us an insight on the signal discovery potential of MINOS for 2 years of running, should the true MDC parameters be nature's choice, whereas the latter allows us to probe the ability of this type of analysis to identify a signal-should it exist-if abundant statistics is available. The $\chi^{2}$ curves obtained for i) and iii) and the curves at the best fit values found from ii) and iv) are shown in Fig. 5.3. From the figure, it is clear that for both intensities a considerably better fit is obtained when the NEUGEN parameters are allowed to stray from the nominal values in the Far+Near case. The effect is less pronounced when looking at the Far only fits.

Some additional information can be be gained by plotting together the $\Delta \chi^{2}$ curves obtained from the fits. This representation is illustrated in Fig. 5.4. The figure shows that the addition of the Near Detector improves to some extent (narrower allowed 

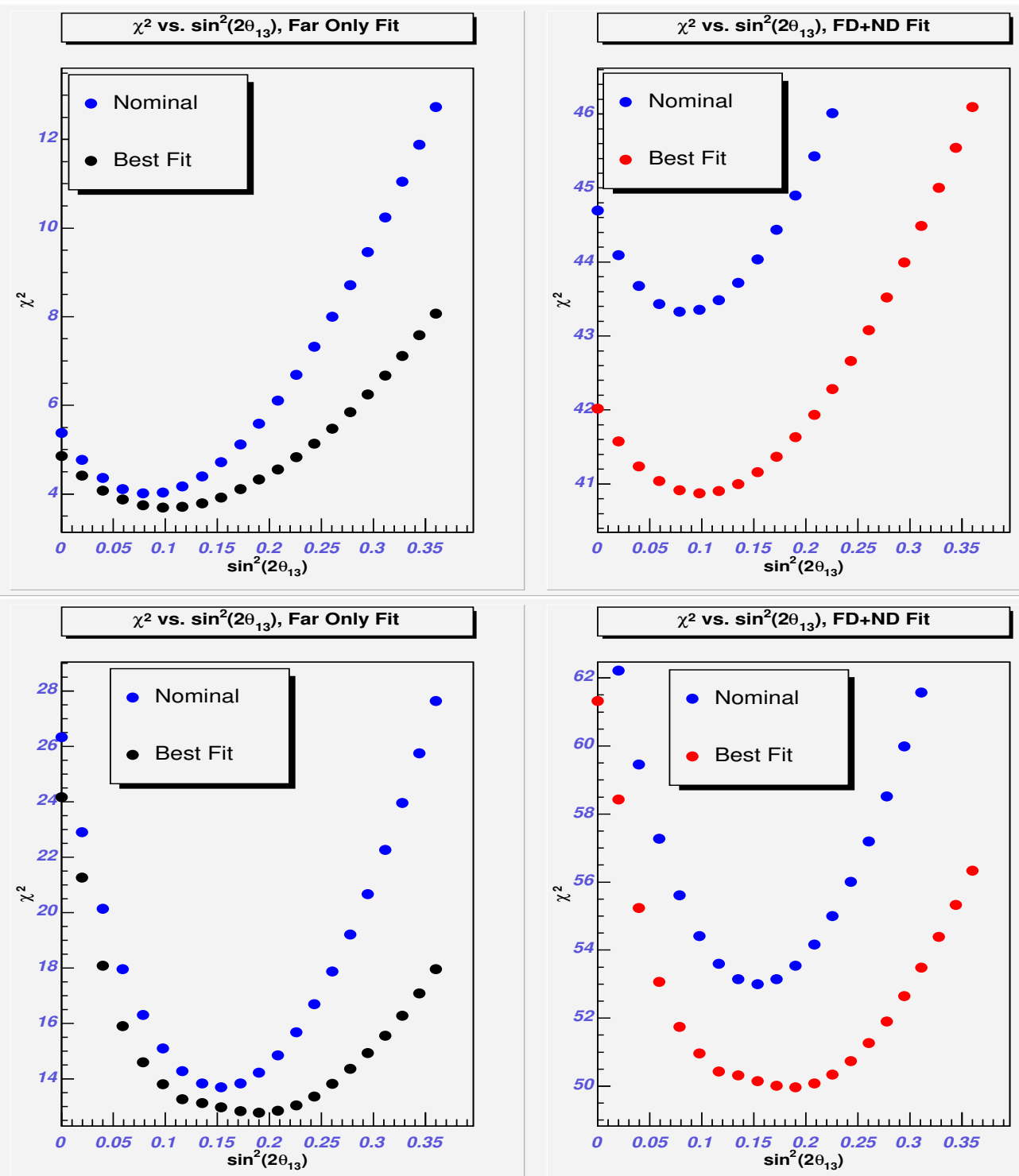

Figure 5.3: $\chi^{2}$ vs. $\sin ^{2}\left(2 \theta_{13}\right)$ curves obtained from MDC fits for two exposures. The top plots show results for $7.4 \times 10^{20}$ POT and the bottom plots for $22.2 \times 10^{20}$ POT. The Far Detector only fit results, for 20 degrees of freedom, are depicted on the left. The Far+Near Detector results, for 20+20 degrees of freedom, are shown on the right.

regions for equivalent confidence levels) the uncertainties introduced by letting the NEUGEN and oscillation parameters vary. Since the Near Detector fit is not affected by the oscillation parameter variation, the result is due to the ability of the ND fit to constrain the NEUGEN parameters. It is likely the limited improvement arises 


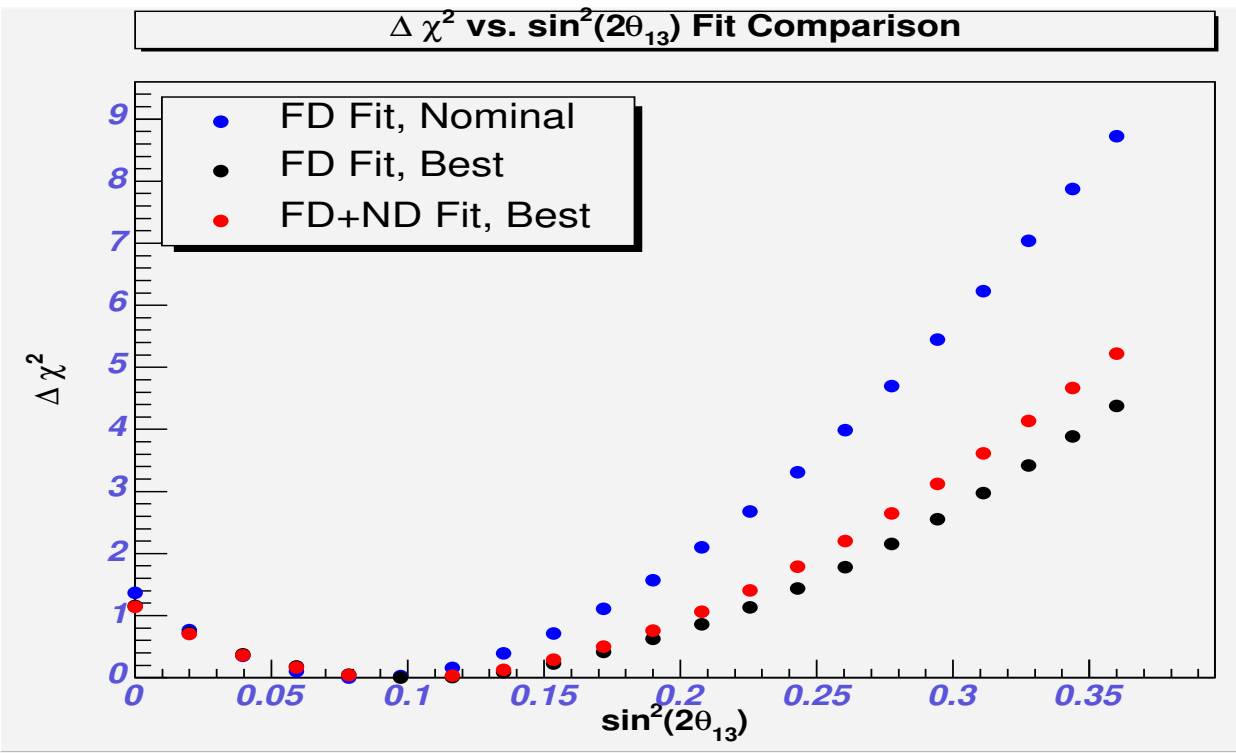

$\Delta \chi^{2}$ vs. $\sin ^{2}\left(2 \theta_{13}\right)$ Fit Comparison

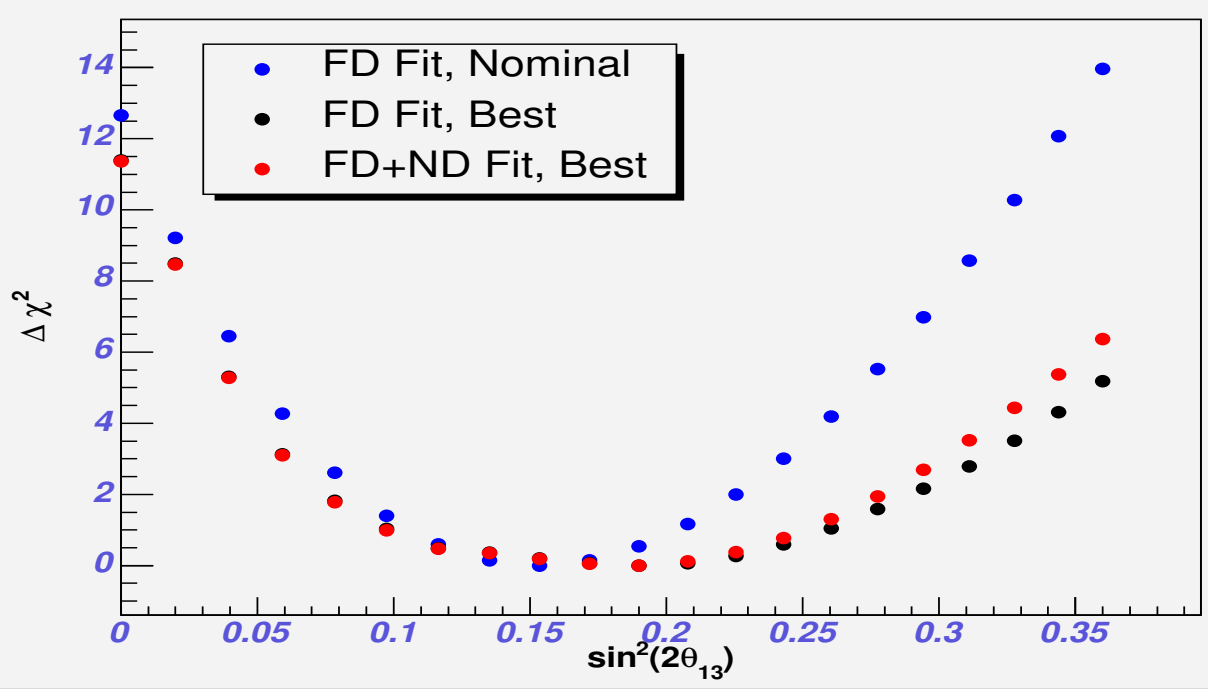

Figure 5.4: $\Delta \chi^{2}$ vs. $\sin ^{2}\left(2 \theta_{13}\right)$ curves obtained from MDC fits for two exposures. The top plot shows results for $7.4 \times 10^{20}$ POT, whereas the bottom plot shows the $22.2 \times 10^{20}$ POT results. The nominal FD+ND fit curve coincides with the nominal FD only fit and is omitted for clarity.

because the effect of the NEUGEN systematics is relatively small compared to the effects of the MINOS CC group measured oscillation parameters. This fact may be inferred from looking at the visible energy spectra of the Far and Near Challenge sets and of their corresponding fits, presented in Fig. 5.5. In the case of the Near 

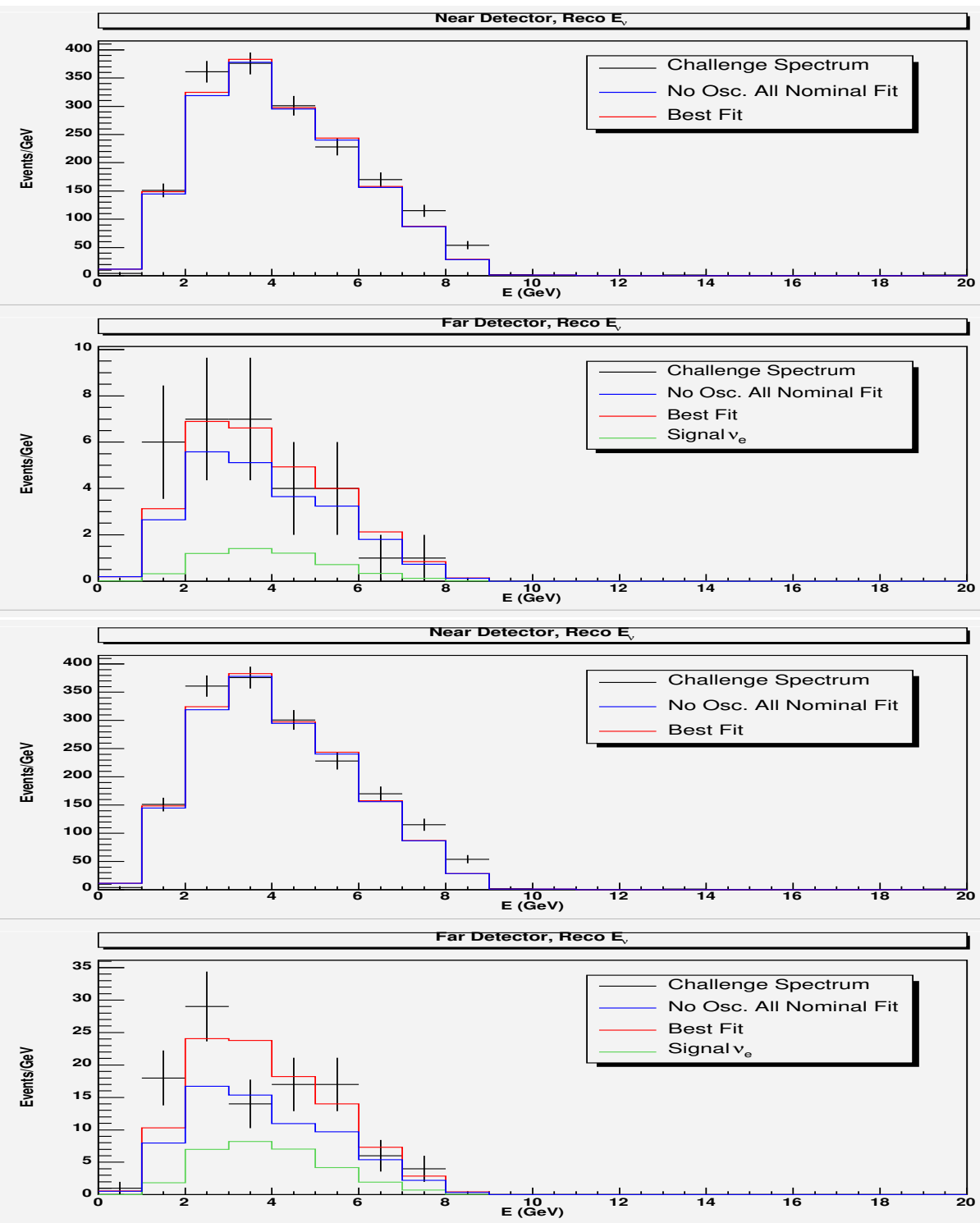

Figure 5.5: MDC fit visible energy spectra for two exposures. The top two plots show the Near and Far spectra for $7.4 \times 10^{20}$ POT, whereas the bottom two plots show the $22.2 \times 10^{20}$ POT spectra. The blue histograms depict the $\sin ^{2}\left(2 \theta_{13}\right)=0$ fit spectra. The expected signal energy distribution is shown in the Far Detector plots as the green histogram.

detector spectrum, for both intensities, the fit with nominal values is very close to that obtained when all parameters are allowed to vary, implying that the variation of the NEUGEN parameters does not greatly affect the Near fit. 
The plots also show a sensible difference between the 2 year and 6 year Far Challenge selected spectra in the 3-4 GeV bin, which may correspond to statistical fluctuations in the two other files that compose the 6 year sample. The Far spectra for 2 years display similar results for the best fit and the no-oscillation solution, whereas the 6 year Far plot shows a much stronger indication of the presence of a $\nu_{\mu} \rightarrow \nu_{e}$ signal.

The ND+FD best fit $\Delta \chi^{2}$ curves with defined 1D $90 \%\left(\Delta \chi^{2} \leqslant 2.71\right)$ and $99 \%$ $\left(\Delta \chi^{2} \leqslant 6.36\right)$ confidence levels are plotted in Fig. 5.6. The 2 year plot depicts a best fit value of $\sin ^{2}\left(2 \theta_{13}\right) \sim 0.10$, but the $90 \%$ confidence interval is clearly consistent with the absence of a $\nu_{e}$ appearance signal. When we increase the available statistics three-fold, there is a very strong indication of the presence of a signal, both at the $90 \%$ and $99 \%$ confidence levels. In fact, the 6 year curve excludes a null oscillation result to a level of $\sim 3.2 \sigma$. The difference obtained for the best fit values is most likely a consequence of the difference in the selected energy spectra for the two intensities. However, it is also apparent that in the 6 year plot a very flat region $\left(0.11<\sin ^{2}\left(2 \theta_{13}\right)<0.21\right)$ occurs, implying that small fluctations could easily shift the best fit value within that region. These results will be compared with the MDC true $\sin ^{2}\left(2 \theta_{13}\right)$ value in the next Section.

The best fit values obtained for all the parameters are condensed in Table 5.5.

\begin{tabular}{|c|c|c|}
\hline & $7.4 \times 10^{20} \mathrm{POT}$ & $22.2 \times 10^{20} \mathrm{POT}$ \\
\hline \hline$\chi_{n o-\text { osc }}^{2} / \mathrm{NDF}$ & $42.0 / 34$ & $61.3 / 34$ \\
$\chi_{\min }^{2} / \mathrm{NDF}$ & $40.9 / 34$ & $49.9 / 34$ \\
\hline QEL_MA & 1.03 & 1.03 \\
RES_MA & 1.03 & 1.03 \\
KNO & 1.04 & 1.04 \\
\hline$\Delta m_{23}^{2}\left(\mathrm{eV}^{2}\right)$ & $2.02 \times 10^{-3}$ & $2.02 \times 10^{-3}$ \\
$\sin ^{2}\left(2 \theta_{23}\right)$ & 0.860 & 0.860 \\
$\sin ^{2}\left(2 \theta_{13}\right)$ & 0.097 & 0.190 \\
\hline
\end{tabular}

Table 5.5: Best fit values for the parameters used in the MDC fit for two different intensities. The rows corresponding to NEUGEN parameters show the determined weights. The $\chi^{2}$ value obtained for no $\nu_{\mu} \rightarrow \nu_{e}$ oscillations is included for reference. 


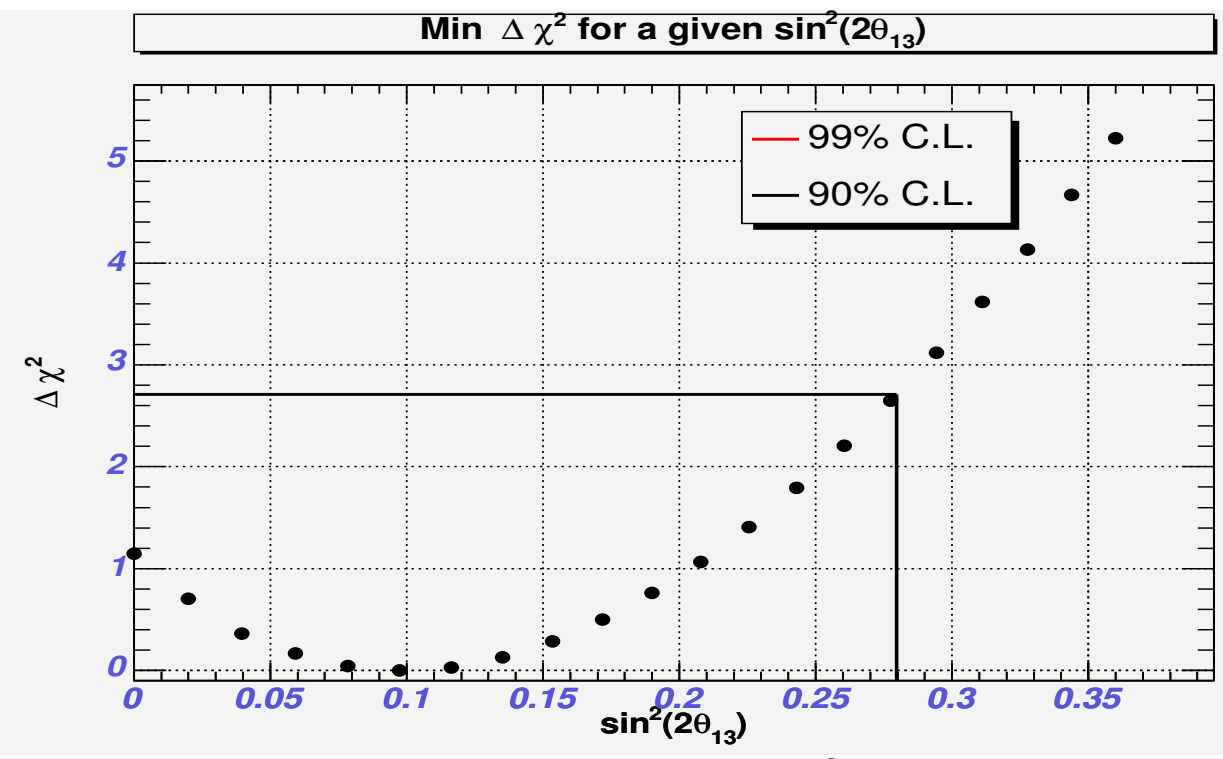

Min $\Delta \chi^{2}$ for a given $\sin ^{2}\left(2 \theta_{13}\right)$

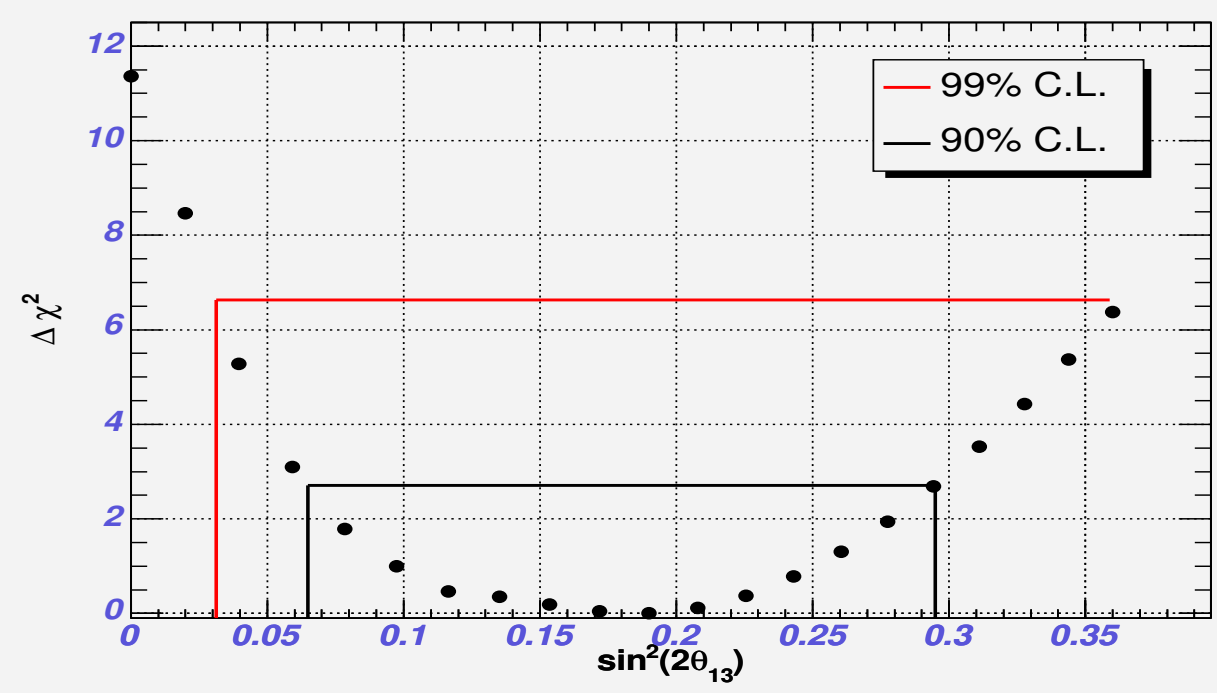

Figure 5.6: 1D 90\% and 99\% confidence intervals for the MDC FD+ND best fit using two exposures. The top two plots displays the result for $7.4 \times 10^{20}$ POT, whereas the bottom plot shows the $22.2 \times 10^{20}$ POT equivalent. In the top plot the $99 \%$ confidence interval is not displayed, as it emcompasses the complete range of $\sin ^{2}\left(2 \theta_{13}\right)$ values used in the fit. In the bottom plot, the $99 \%$ confidence interval stops at $\sin ^{2}\left(2 \theta_{13}\right)=0.36$, as there is no data available above that value. Assuming the $\Delta \chi^{2}$ continues to follow the same trend for higher abscissa values, the 99\% C.L. should close at $\sin ^{2}\left(2 \theta_{13}\right) \sim 0.37$. 


\subsection{True Parameters}

During the June 2005 MINOS collaboration meeting, a year and a half after the Mock Data Challenge was originally proposed, the complete set of parameters used to generate the Challenge set was disclosed. The true values of the parameters relevant for the analysis of this thesis are summarized in Table 5.6.

\begin{tabular}{|c|c|c|}
\hline & MDC Truth & MDA Best Fit \\
\hline \hline QEL_MA $\left(\mathrm{GeV} / \mathrm{c}^{2}\right)$ & 1.012 & 1.062 \\
RES_MA $\left(\mathrm{GeV} / \mathrm{c}^{2}\right)$ & 1.060 & 1.062 \\
KNO & 1.025 & 1.040 \\
\hline$\Delta m_{23}^{2}\left(\mathrm{eV}^{2}\right)$ & $2.12 \times 10^{-3}$ & $2.02 \times 10^{-3}$ \\
$\sin ^{2}\left(2 \theta_{23}\right)$ & 0.881 & 0.860 \\
$\sin ^{2}\left(2 \theta_{13}\right)$ & 0.152 & 0.190 \\
\hline
\end{tabular}

Table 5.6: The MDC true values used in the generation of the Mock Data samples. The results of the MDA best fit to the Challenge set for $22.2 \times 10^{20}$ POT are shown for reference and easy comparison. The NEUGEN weights determined in the best fit were multiplied by the NEUGEN default values for clarity.

The direct comparison of the MDC true value for $\sin ^{2}\left(2 \theta_{13}\right)$ with the MDA best fit value requires some care, as the best fit value of $\sin ^{2}\left(2 \theta_{23}\right)$ used to perform the fits differs slightly from the true value in the MDC. In the One-Mass-Scale approximation, described in Chapter 1 , where we have $\Delta m_{12}^{2} \ll \Delta m_{23}^{2} \approx \Delta m_{13}^{2}$, we may write:

$$
P\left(\nu_{\mu} \rightarrow \nu_{e}\right)=\sin ^{2}\left(\theta_{23}\right) \sin ^{2}\left(2 \theta_{13}\right) \sin ^{2}\left(\Delta m^{2} L / 4 E\right) .
$$

We may thus infer that the $\sin ^{2}\left(2 \theta_{13}\right)$ axis shown in the best fit plots was effectively scaled by a factor of $\sin ^{2}\left(\theta_{23_{\mathrm{MDA}}}\right) / \sin ^{2}\left(\theta_{23_{\mathrm{MDC}}}\right)$, with $\theta_{23_{\mathrm{MDA}}}=0.594$ and $\theta_{23_{\mathrm{MDC}}}=$ 0.609. Therefore, on the best fit plot axis, the true MDC value for $\sin ^{2}\left(2 \theta_{13}\right)$ should correspond to:

$$
\sin ^{2}\left(2 \theta_{13}\right)_{M D C} \times \frac{\sin ^{2}\left(\theta_{23_{\mathrm{MDC}}}\right)}{\sin ^{2}\left(\theta_{23_{\mathrm{MDA}}}\right)}=0.152 \times 1.046=0.159 .
$$

As mentioned throughout the text, other analyses were carried out within the $\nu_{e}$ analysis group with the goal of completing the Mock Data Challenge, namely the Decision Trees (DT) analysis at Harvard and the Artificial Neural Network (ANN) 
analysis at Stanford. Fig. 5.7 presents the final results obtained by the three analyses and compares them with the $\mathrm{CHOOZ}$ limit and the $\mathrm{MDC}$ true value. All three
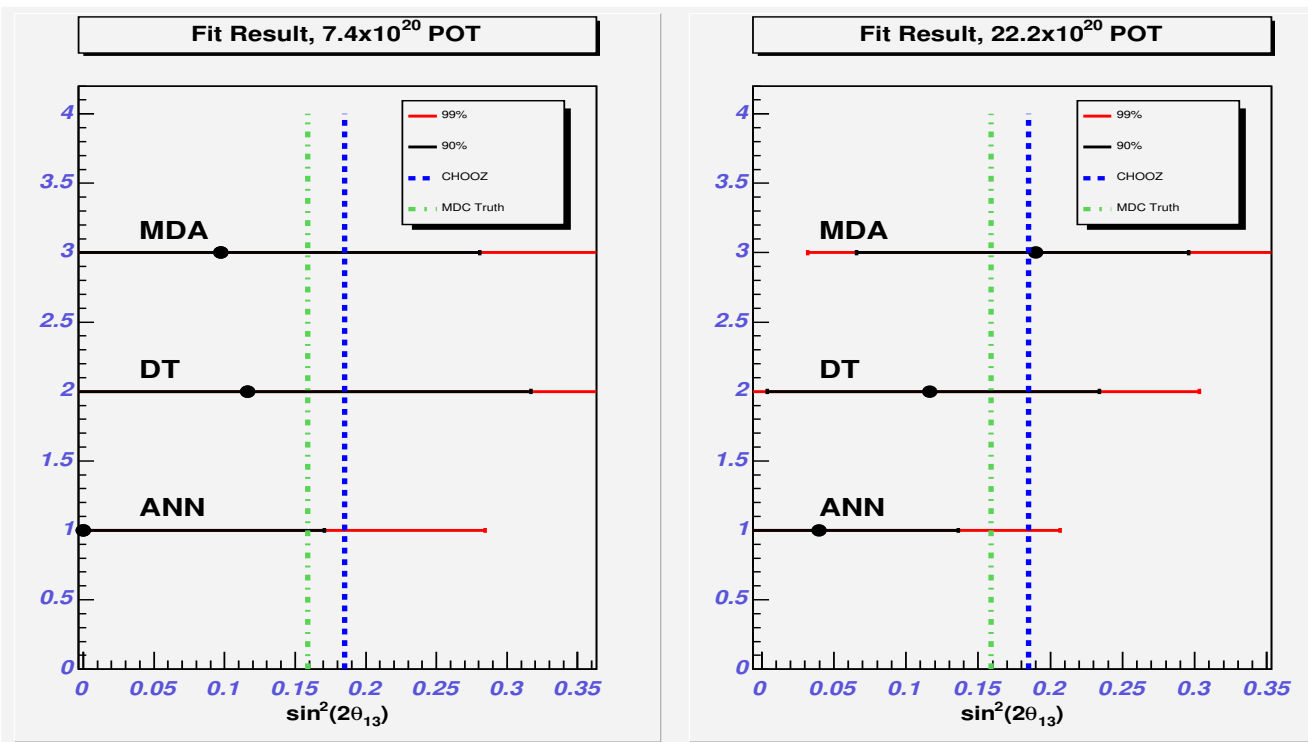

Figure 5.7: Comparison of the results of the three analyses that completed the Mock Data Challenge. The solid black and red lines represent the $90 \%$ and $99 \%$ confidence levels, respectively. The CHOOZ $90 \%$ limit is depicted as the dashed blue line and the scaled MDC true value is shown as the dot-dashed green line.

analyses yield similar results for 2 year statistics. However, for the 6 year Mock Data sample the MDA method presents the most unequivocal evidence for existence of a $\nu_{e}$ appearance signal. It is reassuring to note that three algorithmically different methods obtain consistent results.

\subsection{Concluding Remarks}

It has been shown in this thesis that the multivariate discriminant method employed was very successful in identifying a $\nu_{\mu} \rightarrow \nu_{e}$ oscillation signal in the Mock Data set and thus constitutes a valid option for event classification of the MINOS data sample when significant statistics are accumulated.

The Mock Data Challenge was instrumental in the unambiguous development and testing of analysis techniques for a strongly statistically limited measurement such 
as the $\nu_{e}$ appearance one. It should be noted that the true Challenge set values of the "atmospheric" oscillation parameters are not particularly favorable to $\nu_{\mu} \rightarrow \nu_{e}$ oscillations, as the low value of $\Delta m_{23}^{2}$ and $\theta_{23}$ away from maximal mixing decrease the $\nu_{\mu} \rightarrow \nu_{e}$ oscillation probability. A set of values closer to the best fit of SuperK could improve the signal $/ \sqrt{b g}$ factor by $\sim 50 \%$. It is nevertheless clear from the results for a $7.4 \times 10^{20}$ POT intensity that the MINOS experiment will have a low chance of observing a $\nu_{e}$ appearance oscillation signal in only 2 years of running, even if the $\theta_{13}$ value is right below the $\mathrm{CHOOZ}$ limit. On the other hand, MINOS should be able to set the best world limit even with limited statistics and continue to produce the leading results in the field until the onset of next generation reactor experiments, such as Double-CHOOZ and second generation long baseline experiments, such as NOvA and T2K.

The results shown for event classification in Chapter 4 indicate that improvements are still possible in separating electromagnetic and hadronic showers. This will require devising new discriminator variables or improvements on the existing ones, which could be achieved by a limited iteration between automated classification and visual scanning of events.

A credible MINOS $\nu_{e}$ appearance measurement will have to rely on a thorough understanding of the intrinsic $\nu_{e}$ component of the NuMI beam and of its correct extrapolation to the Far Detector. This may be achieved by using the Near Detector, results from the MIPP experiment [105], which is in the process of studying in detail hadron production in the NuMI target, and the future MINERvA experiment [106], which has the potential to make great improvements on the global knowledge of neutrino cross-sections.

With the Mock Data Challenge now completed, the next challenge will be the real data itself. 


\section{Appendix A}

\section{Mock Data Challenge Reco Scripts}

\section{A.1 Far Detector Monte Carlo Script}

void reco_MC_far(int lastPlane=485, int runNumber) \{

// Script for Farm MC Production.

// NtpSR and NtpMC trees will be out of sync. Need to follow Sue's

// procedure to work with them.

// Note that the entry point name must also be the element name.

// I do this by using a "generic" name and copying the "real" routine

// to a local version with the "generic" name.

// There are 2 runtime arguments:

// lastPlane: The number of planes installed

// runNumber: The run number

// This is used as:

// cp path/reco_far_R1.12.C reco_MC_far.C

// loon -bq "reco_MC_far.C(lastPlane, runNumber)" <MC_file>

//Link dynamic libraries

gSystem->Load ("libDataUtil");

gSystem->Load ("libRecoBase");

gSystem->Load ("libNoiseFilter");

gSystem->Load ("libDetSim");

gSystem->Load ("libPhotonTransport");

gSystem->Load("libBField");

gSystem->Load ("libNumericalMethods");

gSystem->Load ("libSwimmer") ;

gSystem->Load ("libDeMux");

gSystem->Load ("libCandStripSR");

gSystem->Load ("libCandSliceSR");

gSystem->Load ("libCandTrackSR");

gSystem->Load ("libCandClusterSR");

gSystem->Load ("libCandShowerSR"); 


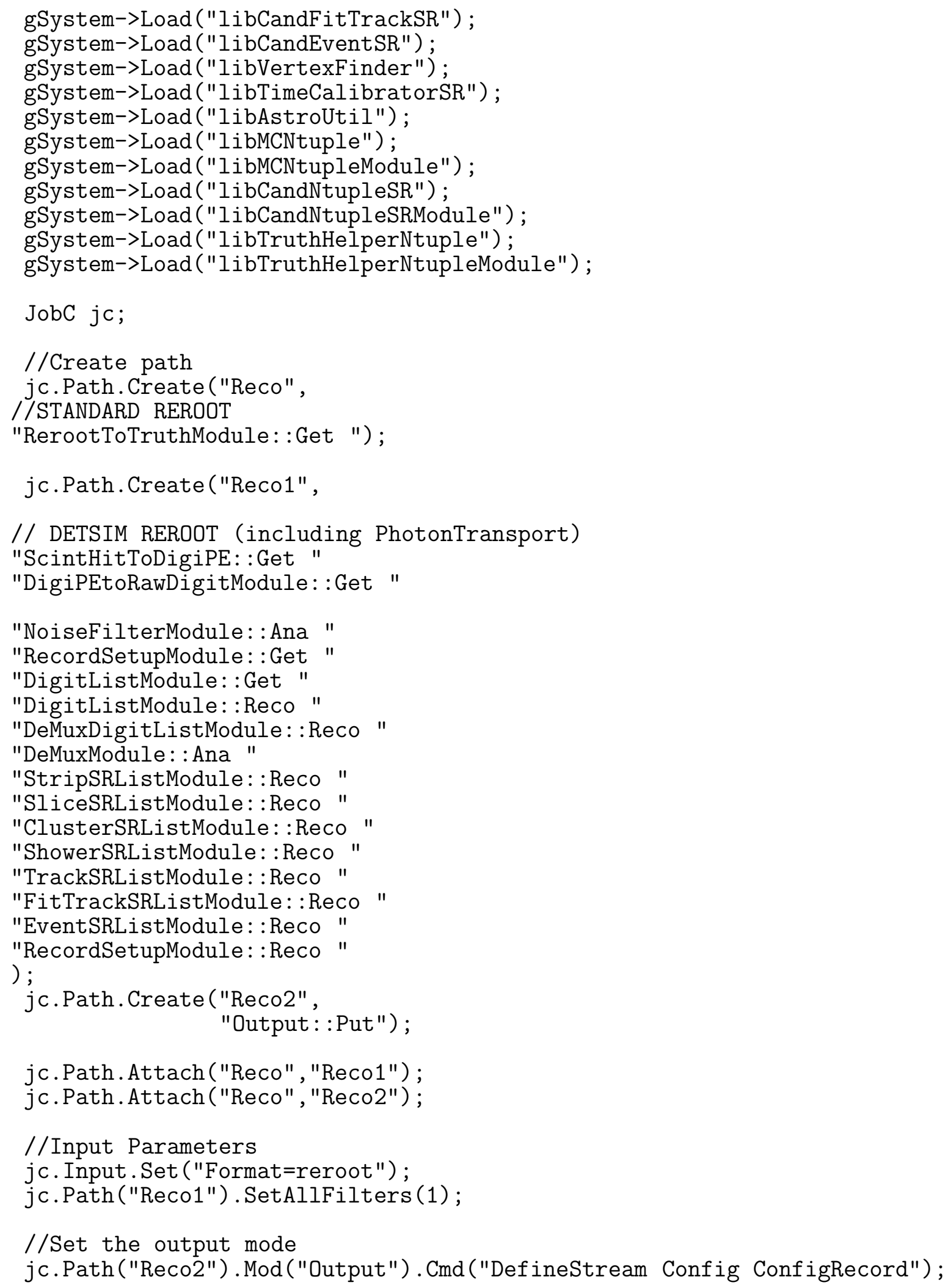


jc.Path ("Reco2") . Mod ("Output") . Set ("Streams=SimSnarl, Cand ,Config, DaqSnarl");

$1 / / / / / / / / / / / / / / / / / / / / / / / / / / / / / / / / / / /$

//Configure Reconstruction Software for Far Det Beam data.

jc.Path ("Reco1"). Mod ("SliceSRListModule") . Set ("SliceListAlgConfig=FarBeam");

jc.Path("Reco1") .Mod ("ClusterSRListModule") . Set ("ClusterListAlgConfig=FarBeam");

jc.Path ("Reco1") . Mod ("ShowerSRListModule") . Set ("ShowerListAlgConfig=FarBeam");

jc.Path ("Reco1") . Mod ("TrackSRListModule") . Set ("TrackListAlgConfig=FarBeam");

jc.Path("Reco1").Mod ("FitTrackSRListModule") . Set ("FitTrackListAlgConfig=FarBeam"

jc.Path("Reco1"). Mod ("EventSRListModule") . Set ("EventListAlgConf ig=FarBeam");

l///////////////////////////////////

// Set up RerootExodus to build the event as MC (instead of Reroot)

RerootExodus: : SetVldSimFlag (SimFlag: :kMC);

// Set the simulated date of the events as to allow for consistent calibration

// RerootExodus: : SetOverrideVldTimeStamp();

// Set Ugli to use only database.

UgliLoanPool: : SetAlwaysUseDbi(true);

// Get the AlgFactory

AlgFactory \\&af = AlgFactory: :GetInstance () ;

// AlgDeMuxDigitList AlgConfig parameters

AlgHandle ah = af.GetAlgHandle("AlgDeMuxDigitList", "default");

AlgConfig \\&acd $=$ ah.GetAlgConfig () ;

acd. UnLockValues ();

acd.Set("DeMuxAlgorithm", "AlgDeMuxBeam"); //Configure DeMuxer for beam data. acd.Set ("DeMuxAlgConfig", "default");

acd.Set("NormalizeWeights", 1); // Normalize weights to 1 if non-zero acd.Set("TrimHyps", 1); // Drop "0" weights if neg., or keep top N acd.LockValues ();

//DigitListModule parameters

//Write both canddigitlist and candmcdigitlist.

jc.Path ("Reco1").Mod ("DigitListModule") . Set ("ListsToMake=3");

jc.Path("Reco1").Mod("StripSRListModule"). Set ("ListIn=canddigitlist");

//BegPlane and EndPlane are now Registry keys.

AlgHandle alh = af.GetAlgHandle("AlgStripSRList", "default");

AlgConfig l\&alg = alh.GetAlgConfig( $)$;

alg.UnLockValues();

alg.Set ("BegPlane" ,1);

alg.Set ("EndPlane", 485);

alg.LockValues ();

//Ntuple record has its own output file so needs its own output module jc.Path.Create ("NtpSR",

"NtpSRModule: : Reco "

"NtpMCModule::Reco " //Necessary to write the MC info tree 


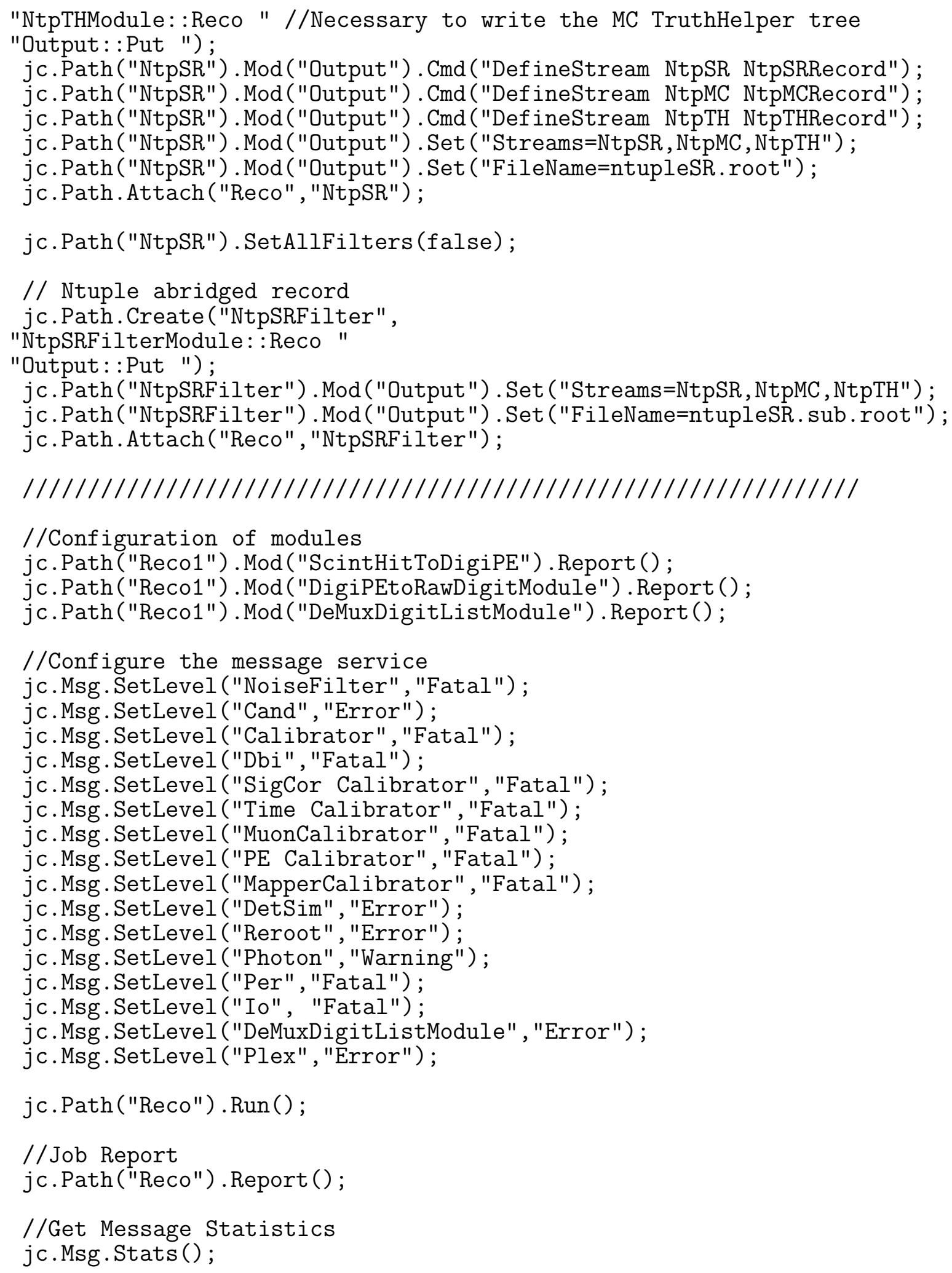




\section{A.2 Far Detector Mock Data Script}

void reco_MC_fmock(int lastPlane=485, int runNumber) \{

// Script for Farm MC Production.

// NtpSR and NtpMC trees will be out of sync. Need to follow Sue's

// procedure to work with them.

// Note that the entry point name must also be the element name.

// I do this by using a "generic" name and copying the "real" routine

// to a local version with the "generic" name.

// There are 2 runtime arguments:

// lastPlane: The number of planes installed

// runNumber: The run number

// This is used as:

// cp path/reco_fmock_R1.12.C reco_MC_fmock.C

// loon -bq "reco_MC_fmock.C(lastPlane, runNumber)" <MC_file>

//Link dynamic libraries

gSystem->Load ("libDataUtil");

gSystem->Load ("libRecoBase");

gSystem->Load ("libNoiseFilter");

gSystem->Load("libDetSim");

gSystem->Load ("libPhotonTransport");

gSystem->Load ("libBField");

gSystem->Load ("libNumericalMethods");

gSystem->Load ("libSwimmer") ;

gSystem->Load ("libDeMux");

gSystem->Load ("libCandStripSR");

gSystem->Load ("libCandSliceSR");

gSystem->Load ("libCandTrackSR");

gSystem->Load ("libCandClusterSR") ;

gSystem->Load ("libCandShowerSR");

gSystem->Load ("libCandFitTrackSR") ;

gSystem->Load ("libCandEventSR");

gSystem->Load ("libVertexFinder");

gSystem->Load ("libTimeCalibratorSR") ;

gSystem->Load ("libAstroUtil");

gSystem->Load ("libMCNtuple");

gSystem->Load ("libMCNtupleModule");

gSystem->Load ("libCandNtupleSR") ;

gSystem->Load ("libCandNtupleSRModule");

gSystem->Load ("libTruthHelperNtuple");

gSystem->Load ("libTruthHelperNtupleModule"); 


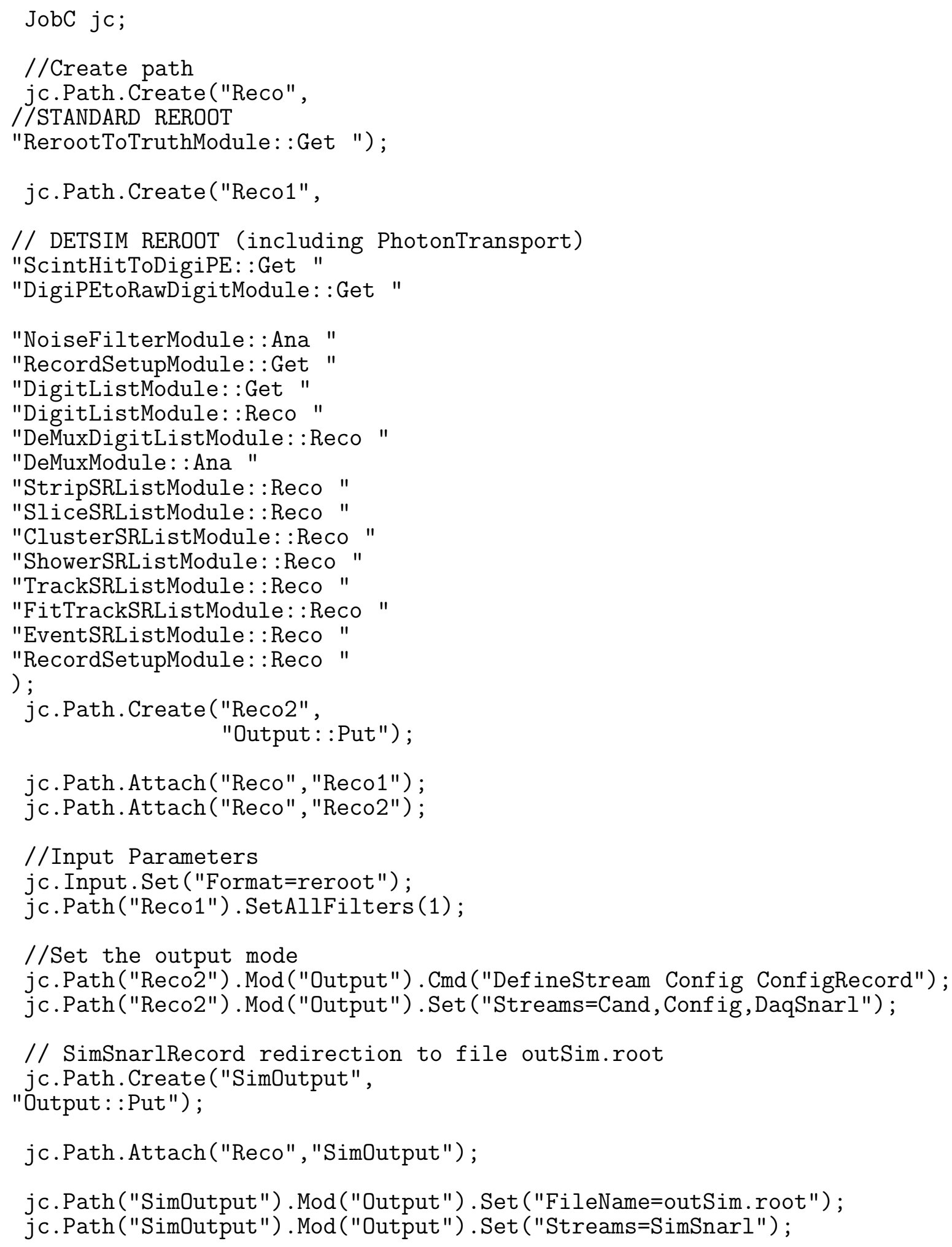


//Configure Reconstruction Software for Far Det Beam data .

jc.Path ("Reco1"). Mod ("SliceSRListModule"). Set ("SliceListAlgConfig=FarBeam");

jc.Path ("Reco1"). Mod ("ClusterSRListModule"). Set ("ClusterListAlgConfig=FarBeam");

jc.Path ("Reco1"). Mod ("ShowerSRListModule"). Set ("ShowerListAlgConfig=FarBeam");

jc.Path ("Reco1"). Mod ("TrackSRListModule"). Set ("TrackListAlgConf ig=FarBeam");

jc.Path ("Reco1") . Mod ("FitTrackSRListModule"). Set ("FitTrackListAlgConfig=FarBeam"

jc.Path ("Reco1"). Mod ("EventSRListModule"). Set ("EventListAlgConfig=FarBeam");

//////////////////////////////////

// Set up RerootExodus to build the event as MC (instead of Reroot)

RerootExodus: : SetVldSimFlag (SimFlag: :kMC);

// Set the simulated date of the events as to allow for consistent calibration

// RerootExodus: :SetOverrideVldTimeStamp();

// Set Ugli to use only database.

UgliLoanPool : :SetAlwaysUseDbi (true);

// Get the AlgFactory

AlgFactory \&af = AlgFactory: :GetInstance () ;

// AlgDeMuxDigitList AlgConfig parameters

AlgHandle ah = af.GetAlgHandle("AlgDeMuxDigitList", "default");

AlgConfig \&acd = ah.GetAlgConfig( );

acd.UnLockValues();

acd.Set("DeMuxAlgorithm", "AlgDeMuxBeam"); //Configure DeMuxer for beam data.

acd.Set("DeMuxAlgConfig", "default");

acd.Set("NormalizeWeights", 1); // Normalize weights to 1 if non-zero

acd.Set("TrimHyps", 1); // Drop "0" weights if neg., or keep top N

acd.LockValues();

//DigitListModule parameters

//Write both canddigitlist and candmcdigitlist.

jc.Path ("Reco1"). Mod ("DigitListModule").Set ("ListsToMake=3");

jc.Path ("Reco1").Mod ("StripSRListModule"). Set ("ListIn=canddigitlist");

//BegPlane and EndPlane are now Registry keys.

AlgHandle alh = af.GetAlgHandle("AlgStripSRList", "default");

AlgConfig \&alg = alh.GetAlgConfig ();

alg.UnLockValues ();

alg. Set ("BegPlane", 1);

alg. Set ("EndPlane", 485);

alg.LockValues();

//Ntuple record has its own output file so needs its own output module jc.Path.Create ("NtpSR",

"NtpSRModule: :Reco "

"Output: :Put ");

jc.Path("NtpSR") .Mod("Output"). Cmd("DefineStream NtpSR NtpSRRecord");

jc.Path ("NtpSR").Mod ("Output"). Set ("Streams=NtpSR");

jc.Path("NtpSR").Mod("Output"). Set ("FileName=ntupleSR.root"); 


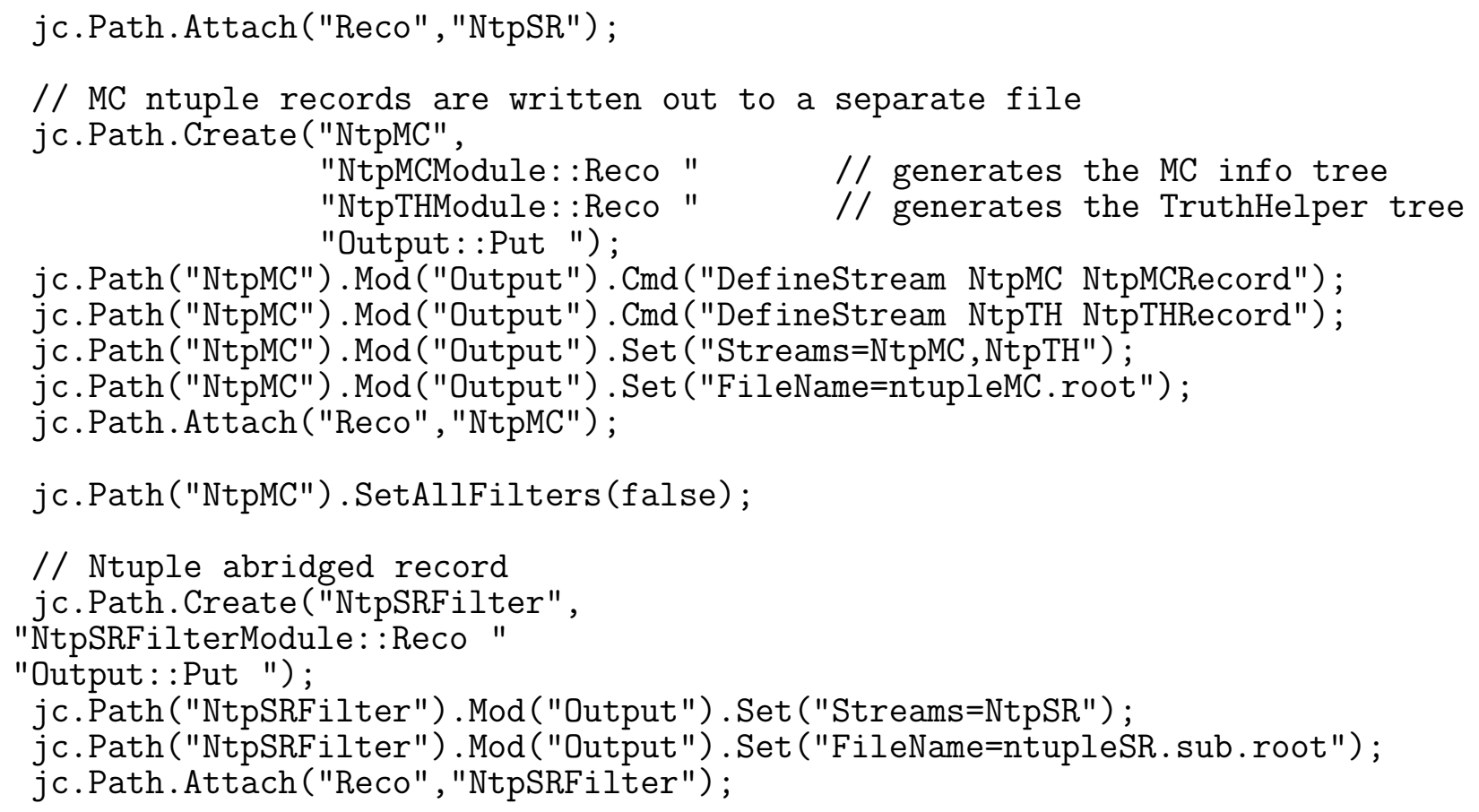


//Get Message Statistics

jc.Msg.Stats();

\}

\section{A.3 Near Detector Monte Carlo Script}

void reco_MC_near(int lastPlane=281, int runNumber) \{

// Script for Farm MC Production.

// Note that the entry point name must also be the element name.

// I do this by using a "generic" name and copying the "real" routine

// to a local version with the "generic" name.

// There are 2 runtime arguments:

// lastPlane: The number of planes installed

// runNumber: The run number

// This is used as:

// cp path/reco_near_R1.12.C reco_MC_near.C

// loon -bq "reco_MC_near.C(lastPlane, runNumber)" <MC_file>

//Link dynamic libraries

gSystem->Load ("libDataUtil");

gSystem->Load ("libRecoBase");

gSystem->Load ("libNoiseFilter");

gSystem->Load("libDetSim");

gSystem->Load ("libPhotonTransport");

gSystem->Load ("libBField");

gSystem->Load ("libNumericalMethods");

gSystem->Load ("libSwimmer");

gSystem->Load ("libCandStripSR");

gSystem->Load ("libCandSliceSR");

gSystem->Load ("libCandTrackSR");

gSystem->Load ("libCandClusterSR") ;

gSystem->Load ("libCandShowerSR");

gSystem->Load ("libCandFitTrackSR");

gSystem->Load ("libCandEventSR");

gSystem->Load ("libVertexFinder");

gSystem->Load ("libTimeCalibratorSR");

gSystem->Load ("libAstroUtil");

gSystem->Load ("libCandNtupleSR");

gSystem->Load ("libCandNtupleSRModule") ;

gSystem->Load ("libMCNtuple") ;

gSystem->Load ("libMCNtupleModule") ;

gSystem->Load ("libTruthHelperNtuple");

gSystem->Load ("libTruthHelperNtupleModule"); 


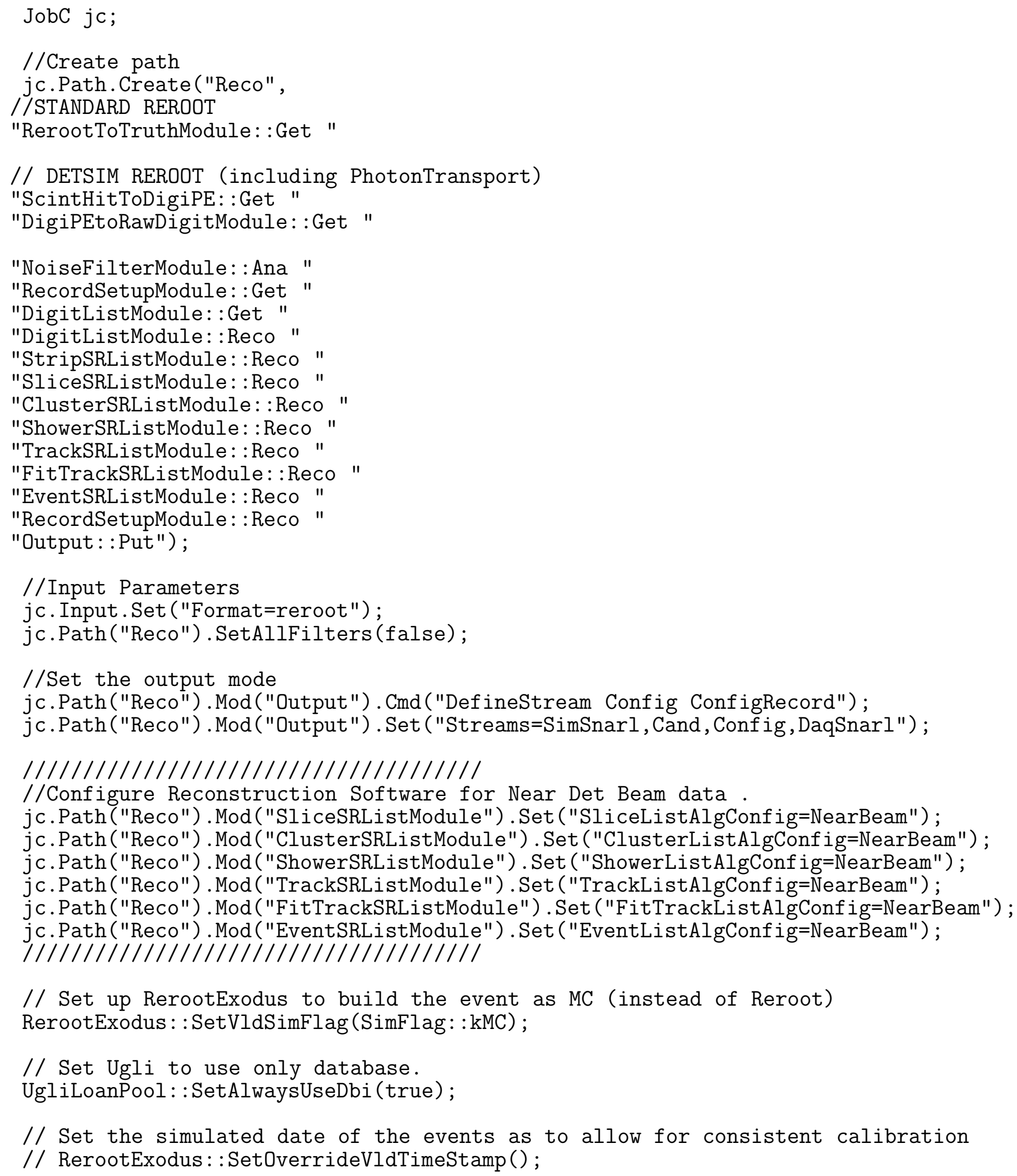


// Get the AlgFactory

AlgFactory \&af = AlgFactory: :GetInstance () ;

// AlgDeMuxDigitList AlgConfig parameters

AlgHandle ah = af.GetAlgHandle("AlgDeMuxDigitList", "default");

AlgConfig \&acd $=$ ah. GetAlgConfig( () ;

acd.UnLockValues ();

acd.Set("NormalizeWeights", 1); // Normalize weights to 1 if non-zero acd.Set("TrimHyps", 1); // Drop "0" weights if neg., or keep top N acd.LockValues ();

//DigitListModule parameters

//Write both canddigitlist and candmcdigitlist.

jc.Path ("Reco").Mod("DigitListModule"). Set ("ListsToMake=3");

jc.Path ("Reco").Mod ("StripSRListModule"). Set ("ListIn=canddigitlist");

//BegPlane and EndPlane are now Registry keys.

AlgHandle alh = af.GetAlgHandle ("AlgStripSRList", "default");

AlgConfig \&alg = alh.GetAlgConfig();

alg. UnLockValues ();

alg.Set ("BegPlane",1);

alg. Set ("EndPlane", 281);

alg.LockValues ();

//Reco's filter parameters

jc.Path ("Reco"). Mod ("StripSRListModule"). Set ("BegPlane=1");

jc.Path ("Reco").Mod ("StripSRListModule"). Set ("EndPlane=281");

//Ntuple record has its own output file so needs its own output module jc.Path.Create ("NtpSR",

"NtpSRModule: :Reco "

"NtpMCModule::Reco "//Necessary to write the MC info tree

"NtpTHModule::Reco "//Necessary to write the MC TruthHelper tree

"Output::Put ");

jc.Path("NtpSR"). Mod("Output"). Cmd("DefineStream NtpSR NtpSRRecord");

jc.Path ("NtpSR"). Mod("Output"). Cmd("DefineStream NtpMC NtpMCRecord");

jc.Path("NtpSR"). Mod("Output"). Cmd("DefineStream NtpTH NtpTHRecord");

jc.Path ("NtpSR"). Mod ("Output"). Set ("Streams=NtpSR, NtpMC , NtpTH") ;

jc.Path ("NtpSR").Mod ("Output"). Set ("FileName=ntupleSR.root");

jc.Path.Attach("Reco", "NtpSR");

// Ntuple abridged record

jc.Path.Create ("NtpSRFilter",

"NtpSRFilterModule:: Reco "

"Output: :Put ");

jc.Path ("NtpSRFilter") . Mod ("Output") . Set ("Streams=NtpSR, NtpMC, NtpTH");

jc.Path ("NtpSRFilter").Mod ("Output"). Set ("FileName=ntupleSR. sub.root");

jc.Path.Attach ("Reco", "NtpSRFilter"); 
//Configuration of modules jc.Path ("Reco") .Mod ("ScintHitToDigiPE").Report();

jc.Path ("Reco"). Mod ("DigiPEtoRawDigitModule"). Report();

//Configure the message service

jc.Msg. SetLevel ("NoiseFilter", "Fatal");

jc.Msg.SetLevel ("Cand", "Error");

jc.Msg.SetLevel ("Calibrator", "Fatal");

jc.Msg.SetLevel ("Dbi", "Fatal");

jc.Msg.SetLevel ("SigCor Calibrator", "Fatal");

jc.Msg.SetLevel ("Time Calibrator", "Fatal");

jc.Msg.SetLevel ("MuonCalibrator", "Fatal");

jc.Msg.SetLevel ("PE Calibrator", "Fatal");

jc.Msg.SetLevel ("MapperCalibrator", "Fatal");

jc.Msg.SetLevel ("DetSim", "Error");

jc.Msg.SetLevel ("Reroot", "Error");

jc.Msg.SetLevel ("Photon", "Warning");

jc.Msg.SetLevel ("Per", "Fatal");

jc.Msg.SetLevel ("Io", "Fatal");

jc.Msg.SetLevel ("Plex", "Error");

jc.Path("Reco").Run();

//Job Report

jc.Path("Reco"). Report();

//Get Message Statistics

jc.Msg.Stats();

\}

\section{A.4 Near Detector Mock Data Script}

void reco_MC_nmock (int lastPlane=281, int runNumber) \{

// Script for Farm MC Production.

// Note that the entry point name must also be the element name.

// I do this by using a "generic" name and copying the "real" routine

// to a local version with the "generic" name.

// There are 2 runtime arguments:

// lastPlane: The number of planes installed

// runNumber: The run number

// This is used as:

// cp path/reco_nmock_R1.12.C reco_MC_nmock.C

// loon -bq "reco_MC_nmock.C(lastPlane, runNumber)" <MC_file> 


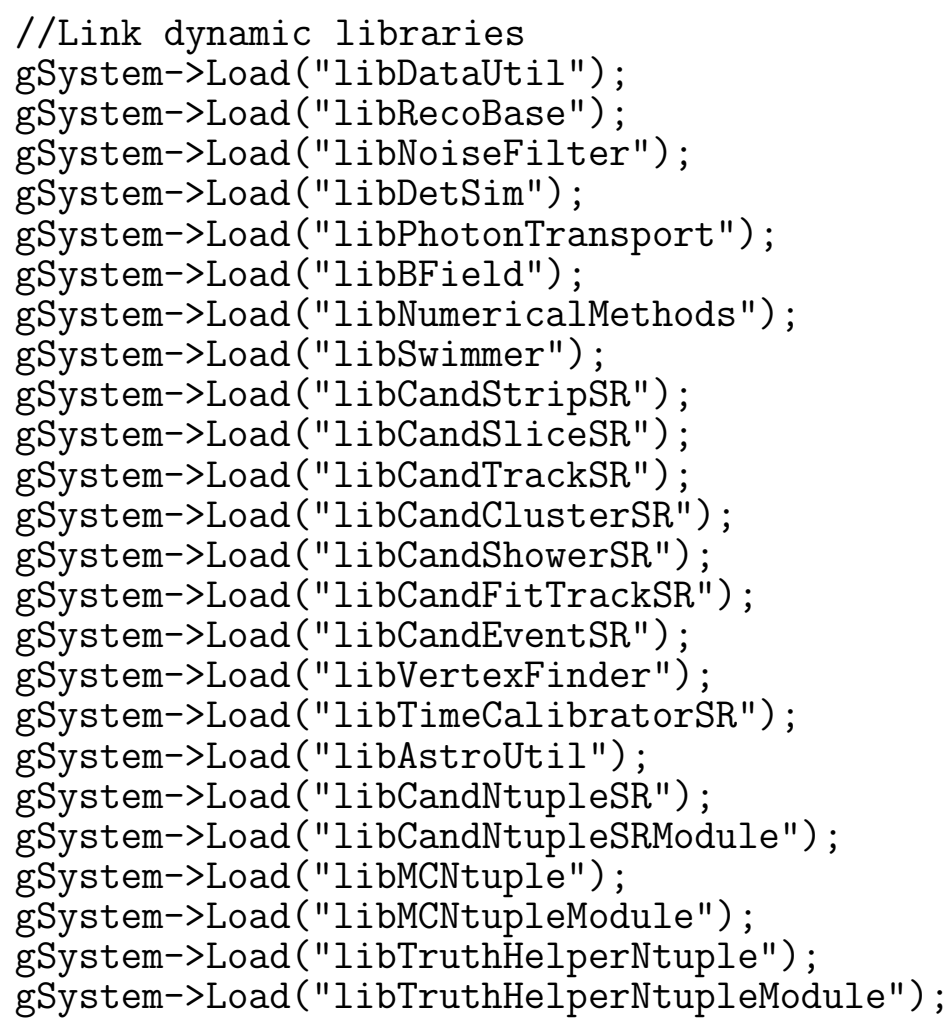


//Input Parameters

jc. Input. Set ("Format=reroot");

jc.Path("Reco"). SetAllFilters(false);

//Set the output mode

jc.Path("Reco").Mod("Output"). Cmd("DefineStream Config ConfigRecord");

jc.Path ("Reco"). Mod ("Output"). Set ("Streams=Cand, Config, DaqSnarl") ;

// SimSnarlRecord redirection to file outSim.root

jc.Path.Create("SimOutput",

"Output: :Put");

jc.Path.Attach("Reco", "SimOutput");

jc.Path ("SimOutput"). Mod ("Output"). Set ("Streams=SimSnarl");

jc.Path ("SimOutput"). Mod("Output"). Set ("FileName=outSim.root");

//////////////////////////////////

//Configure Reconstruction Software for Near Det Beam data.

jc.Path ("Reco").Mod ("SliceSRListModule"). Set ("Sli ceListAlgConfig=NearBeam");

jc.Path ("Reco"). Mod ("ClusterSRListModule"). Set ("ClusterListAlgConfig=NearBeam");

jc.Path ("Reco"). Mod ("ShowerSRListModule"). Set ("ShowerListAlgConfig=NearBeam");

jc.Path ("Reco").Mod ("TrackSRListModule"). Set ("TrackListAlgConfig=NearBeam");

jc.Path ("Reco").Mod ("FitTrackSRListModule"). Set ("FitTrackListAlgConfig=NearBeam");

jc.Path ("Reco").Mod ("EventSRListModule"). Set ("EventListAlgConfig=NearBeam");

//////////////////////////////////

// Set up RerootExodus to build the event as MC (instead of Reroot)

RerootExodus: : SetVldSimFlag (SimFlag: :kMC);

// Set Ugli to use only database.

UgliLoanPool: : SetAlwaysUseDbi (true);

// Set the simulated date of the events as to allow for consistent calibration

// RerootExodus: :SetOverrideVldTimeStamp();

// Get the AlgFactory

AlgFactory \&af = AlgFactory: :GetInstance ();

// AlgDeMuxDigitList AlgConfig parameters

AlgHandle ah = af.GetAlgHandle("AlgDeMuxDigitList", "default");

AlgConfig \&acd = ah.GetAlgConfig( $)$;

acd.UnLockValues ();

acd.Set("NormalizeWeights", 1); // Normalize weights to 1 if non-zero acd.Set("TrimHyps", 1); // Drop "0" weights if neg., or keep top N acd.LockValues ();

//DigitListModule parameters

//Write both canddigitlist and candmcdigitlist.

jc.Path ("Reco").Mod("DigitListModule"). Set ("ListsToMake=3");

jc.Path ("Reco"). Mod ("StripSRListModule"). Set ("ListIn=canddigitlist"); 
//BegPlane and EndPlane are now Registry keys.

AlgHandle alh = af.GetAlgHandle("AlgStripSRList", "default");

AlgConfig \&alg = alh.GetAlgConfig();

alg.UnLockValues ();

alg.Set ("BegPlane", 1);

alg. Set ("EndPlane", 281);

alg.LockValues ();

//Reco's filter parameters

jc.Path ("Reco"). Mod ("StripSRListModule"). Set ("BegPlane=1");

jc.Path ("Reco"). Mod ("StripSRListModule"). Set ("EndPlane=281");

//Ntuple record has its own output file so needs its own output module jc.Path.Create ("NtpSR",

"NtpSRModule: :Reco "

"Output: :Put ");

jc.Path("NtpSR"). Mod("Output"). Cmd("DefineStream NtpSR NtpSRRecord");

jc.Path ("NtpSR").Mod ("Output"). Set ("Streams=NtpSR");

jc.Path("NtpSR"). Mod ("Output"). Set ("FileName=ntupleSR.root");

jc.Path.Attach("Reco", "NtpSR");

// MC ntuple records are written out to a separate file

jc.Path.Create ("NtpMC",

"NtpMCModuleReroot::Reco " // generates the MC info tree

"NtpTHModule::Reco " // generates the TruthHelper tree

"Output: :Put ");

jc.Path ("NtpMC").Mod("Output"). Cmd("DefineStream NtpMC NtpMCRecord");

jc.Path ("NtpMC"). Mod("Output"). Cmd("DefineStream NtpTH NtpTHRecord");

jc.Path ("NtpMC") . Mod ("Output") . Set ("Streams=NtpMC, NtpTH");

jc.Path ("NtpMC"). Mod ("Output"). Set ("FileName=ntupleMC.root");

jc.Path.Attach("Reco", "NtpMC");

// Ntuple abridged record

jc.Path.Create ("NtpSRFilter",

"NtpSRFilterModule::Reco "

"Output: :Put ");

jc.Path ("NtpSRFilter"). Mod ("Output"). Set ("Streams=NtpSR") ;

jc.Path ("NtpSRFilter").Mod ("Output"). Set ("FileName=ntupleSR. sub.root");

jc.Path.Attach ("Reco", "NtpSRFilter");

$1 / / / / / / / / / / / / / / / / / / / / / / / / / / / / / / / / / / / / / / / / / / / / / / / / / / / / / / / / /$

//Configuration of modules

jc.Path ("Reco"). Mod ("ScintHitToDigiPE"). Report();

jc.Path ("Reco"). Mod ("DigiPEtoRawDigitModule"). Report();

//Configure the message service

jc.Msg.SetLevel ("NoiseFilter", "Fatal"); 


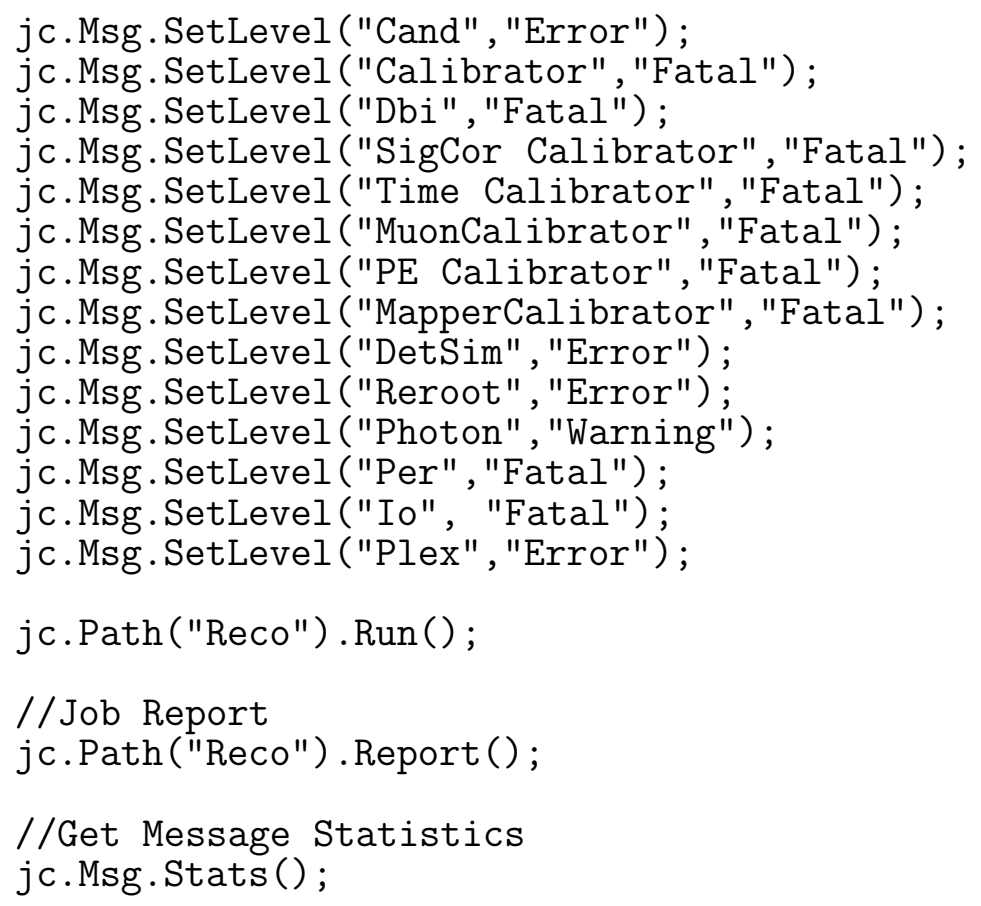




\section{Appendix B}

\section{AnaNue Variable Glossary}

This glossary of all the variables available in the AnaNue ntuple is adapted from the version in Ref. [107]. With exception of the reconstruction variables, brought over from the MINOS Standard ntuple, the variables described here result from the analysis work of J. Boehm and M. Sanchez at Harvard University, T. Yang at Stanford University, P. Vahle at University College London and the author at Tufts University.

\section{Reconstruction Event Variables}

- srevent.phMeu - Event energy

- srevent.planes - Number of planes

- srevent.showers - Number of showers

- srevent.tracks - Number of tracks

- srevent.timeLength - Time length

\section{Reconstruction Shower Variables}

- srshower.phMeu - Shower energy

- srshower.pulseHeightRatio - Shower energy/Event total energy

- srshower.totalStrips - Number of strips

- srshower.stripRatio - Number of strips/Total number of strips

- srshower.planes - Number of planes in the shower (length) 
- srshower.planeRatio - Number of planes/Total number of planes

\section{Reconstruction Track Variables}

- srtrack.phMeu - Track energy

- srtrack.pulseHeightRatio - Track energy/Event total energy

- srtrack.planes - Number of planes in track

- srtrack.trklikePlanes - Number of track-like planes

- srtrack.trklikeRatio - Number of track-like planes/Number of planes in track

- srtrack.totalStrips - Number of strips

4. 3D Hit Variables (Tufts)

- hitcalc.fHitTotalEnergy - Sum of energy of all hits

- hitcalc.fHitTransEnergy - Magnitude of the vectorial sum of the transverse projections of the vectors defined by each hit and the vertex

- hitcalc.fHitLongEnergy - Sum of the magnitudes of the longitudinal projections of the vectors defined as above

- hitcalc.fHitTransCMEnergy - Sum of the squares of the magnitudes of the transverse projections of the vectors defined as above

- hitcalc.fHitTransEnergyRatio - Ratio of hitcalc.fHitTransEnergy to hitcalc.fHitTotalEnergy

- hitcalc.fHitLongEnergyRatio - Ratio of hitcalc.fHitLongEnergy to hitcalc.fHitTotalEnergy

- hitcalc.fHitTransLongEnergyRatio - Ratio of hitcalc.fHitTransEnergy to hitcalc.fHitLongEnergy

- hitcalc.fHitTransCMEnergyRatio - Ratio of hitcalc.fHitTransCMEnergy to hitcalc.fHitTotalEnergy

- hitcalc.fHitFarMomBalance - Ratio of the sum of hit energy on the opposite side of a septum and the sum on the same side, with the septum being defined 
by the perpendicular to the transverse projection of the vector resulting from the difference of the farthest hit in the event to the vertex

- hitcalc.fHitPeakMomBalance - Ratio of the sum of hit energy on the opposite side of a septum and the sum on the same side, with the septum being defined by the perpendicular to the transverse projection of the vector resulting from the difference of the highest energy hit in the event to the vertex

- hitcalc.fHitFarAngle - Angle between the straight line joining the farthest hit with the vertex and the $\mathrm{Z}$ axis, along the incoming neutrino direction

- hitcalc.fHitPeakAngle - Angle between the straight line joining the highest energy hit with the vertex and the $\mathrm{Z}$ axis

\section{Angular Clustering Variables (Tufts)}

- angcluster.fACluRmsShwAxis - RMS value of the radial distribution of the hits belonging to the primary shower/cluster around the primary shower direction

- angcluster.fACluRmsZAxis - RMS value of the radial distribution of the hits belonging to the primary shower/cluster around the $\mathrm{Z}$ axis

- angcluster.fACluShwDirX - X coordinate of the primary shower direction

- angcluster.fACluShwDirY - Y coordinate of the primary shower direction

- angcluster.fACluShwDirZ - Z coordinate of the primary shower direction

\section{Angular Cluster Fitting Variables (Tufts)}

- angclusterfit.fACluFitParA - Longitudinal fit parameter A (shower rise)

- angclusterfit.fACluFitParB - Longitudinal fit parameter B (shower decay)

- angclusterfit.fACluFitParLongE0 - Longitudinal fit parameter E0 (showering particle initial energy)

- angclusterfit.fACluFitShwMax - Longitudinal fit maximum shower value

- angclusterfit.fACluFitE0EnergyRatio - Ratio of angclusterfit.fAcluFitParLongE0 and the total energy contained in the primary shower 3D hits 
- angclusterfit.fACluFitParL1 - Transverse fit parameter L1 (attenuation length in shower core function)

- angclusterfit.fACluFitParL2 - Transverse fit parameter L2 (attenuation length in shower halo function)

- angclusterfit.fACluFitParC12 - Transverse fit parameter C12 (relative weight between shower core and shower halo functions)

- angclusterfit.fACluFitParTransE0 - Transverse fit parameter E0 (showering particle initial energy)

- angclusterfit.fACluFitParL1 - Transverse fit parameter L1 (attenuation length in shower core function)

- angclusterfit.fACluFitLongChiSq - Longitudinal fit chi square

- angclusterfit.fACluFitLongConv - Longitudinal fit convergence

- angclusterfit.fACluFitLongNDF - Longitudinal fit number of degrees of freedom

- angclusterfit.fACluFitTransChiSq - Transverse fit chi square

- angclusterfit.fACluFitTransConv - Transverse fit convergence

- angclusterfit.fACluFitTransNDF - Transverse fit number of degrees of freedom

- angclusterfit.fACluFitAsymPeak - Asymmetry around peak

- angclusterfit.fACluFitAsymVert - Asymmetry around vertex

- angclusterfit.fACluFitMolRadPeak - Molière radius from peak

- angclusterfit.fACluFitMolRadVert - Molière radius from vertex

- angclusterfit.fACluFitMean - Mean of transverse coordinate distribution with respect to primary shower direction

- angclusterfit.fACluFitRMS - RMS of transverse coordinate distribution

- angclusterfit.fACluFitSkew - Skewness of transverse coordinate distribution

- angclusterfit.fACluFitKurt - Kurtosis of transverse coordinate distribution 
7. FracVar Variables (Stanford)

- fracvars.fract_n_planes $(n=1,2,3,4,5,6)$ - The fraction of the observed total energy which is deposited in $n$ consecutive planes with the highest pulse height

- fracvars.fract_n_counters $(n=2,4,6,8,10,12)$ - The fraction of the observed total energy which is deposited in $n$ strips with the highest pulse height

- fracvars.fract_road - The fraction of the observed total energy which is deposited in a narrow road

- fracvars.shw_nstp - The number of strips in the shower core

- fracvars.pid - The output from neural net. A likelihood of being a nue

\section{Minimal Spanning Tree (MST) Variables (UCL)}

- mstvars.e4w, mstvars.o4w - The sum of the weights in the minimal spanning tree formed from the larger than average pulse height hits in the event (the largest primary MST found) in the even or odd view

- mstvars.eb1, mstvars.ob1 - The fraction of the pulse height of hits in the primary MST that are connected by a weight $<10 \mathrm{~cm}$

- mstvars.osmtot - The total pulseheight in MIP that's picked up and put in any MST (in the odd view)

- mstvars.eeprob, mstvars.oeprob - A likelihood of being a nue

- mstvars.ealpha, mstvars.oalpha - A fit parameter, fitting an event distribution of weights to a combination of electron and pion templates (alpha*electron $+(1 \text {-alpha })^{*}$ pion $\left.)\right)$

- mstvars.ebeta, mstvars.obeta - The error on alpha

9. Shower Fit Variables (Harvard)

- shwfit.par_a - Fitted parameter a (shower rise)

- shwfit.par_b - Fitted parameter b (shower fall) 
- shwfit.par_e0 - Fitted parameter $E_{0}$

- shwfit.chisq - Chi square of the fit

- shwfit.chisq_ndf Chi square of the fit per degree of freedom

- shwfit.conv - Convergence of fit (quality of fit)

- shwfit.shwmax - Shower fit maximum

- shwfit.shwmaxplane - Shower fit maximum plane

- shwfit.max_pe_plane - Maximum plane

- shwfit.shwmaxplane_diff - Distance from shower fit maximum plane to maximum plane

- shwfit.e0_pe_ratio - $E_{0} /$ Event total energy

- shwfit.caldet_comp - Comparison of fit to CalDet

\section{Transverse Variables (Harvard)}

Note: the "uv" variables are linear combinations of the corresponding " $u$ " and "v" quantities

- shwfit.uv_asym_peak - Asymmetry around peak

- shwfit.uv_asym_vert - Asymmetry around vertex

- shwfit.uv_molrad_peak - Molière radius from peak

- shwfit.uv_molrad_vertex - Molière radius from vertex

- shwfit.uv_mean - Mean respect to vertex

- shwfit.uv_rms - RMS in coordinate

- shwfit.uv_skew - Skewness in coordinate

- shwfit.uv_kurt - Kurtosis in coordinate 


\section{Bibliography}

[1] W. Pauli, Letter to a physicists' gathering at Tubingen. December 4, 1930, reprinted in Wolfgang Pauli, Collected scientific papers, edited by R. Kronig and V. Weisskopf, vol. 2, p. 1313, New York, Interscience, 1964.

[2] F. Reines and C. L. Cowan, Detection of the free neutrino, Phys. Rev. 92, 830-831 (1953).

[3] M. Goldhaber, L. Grodzins, and A. W. Sunyar, Helicity of neutrinos, Phys. Rev. 109, 1015-1017 (1958).

[4] G. Danby et al., Observation of high-energy neutrino reactions and the existence of two kinds of neutrinos, Phys. Rev. Lett. 9, 36-44 (1962).

[5] K. Kodama et al., Observation of tau-neutrino interactions, Phys. Lett. B504, 218-224 (2001).

[6] B. Pontecorvo, Mesonium and antimesonium, Sov. Phys. JETP 6, 429 (1957).

[7] Y. Fukuda et al., Evidence for oscillation of atmospheric neutrinos, Phys. Rev. Lett. 81, 1562-1567 (1998).

[8] M. C. Sanchez et al., Observation of atmospheric neutrino oscillations in Soudan 2, Phys. Rev. D68, 113004 (2003).

[9] Q. R. Ahmad et al., Direct evidence for neutrino flavor transformation from neutral-current interactions in the Sudbury Neutrino Observatory, Phys. Rev. Lett. 89, 011301 (2002). 
[10] E. Fermi, An attempt of a theory of beta radiation. 1, Z. Phys. 88, 161-177 (1934).

[11] T. D. Lee and C.-N. Yang, Question of parity conservation in weak interactions, Phys. Rev. 104, 254-258 (1956).

[12] C. S. Wu, E. Ambler, R. W. Hayward, D. D. Hoppes, and R. P. Hudson, Experimental test of parity conservation in beta decay, Phys. Rev. 105, 14131414 (1957).

[13] E. C. G. Sudarshan and R. e. Marshak, Chirality invariance and the universal Fermi interaction, Phys. Rev. 109, 1860-1862 (1958).

[14] R. P. Feynman and M. Gell-Mann, Theory of the Fermi interaction, Phys. Rev. 109, 193-198 (1958).

[15] R. P. Feynman, Mathematical formulation of the quantum theory of electromagnetic interaction, Phys. Rev. 80, 440-457 (1950).

[16] S. L. Glashow, Partial symmetries of weak interactions, Nucl. Phys. 22, 579588 (1961).

[17] S. Weinberg, A model of leptons, Phys. Rev. Lett. 19, 1264-1266 (1967).

[18] A. Salam and J. C. Ward, Electromagnetic and weak interactions, Phys. Lett. 13, 168-171 (1964).

[19] M. Gell-Mann, Nonleptonic weak decays and the eightfold way, Phys. Rev. Lett. 12, 155-156 (1964).

[20] M. Y. Han and Y. Nambu, Three-triplet model with double SU(3) symmetry, Phys. Rev. 139, B1006-B1010 (1965).

[21] D. J. Gross and F. Wilczek, Asymptotically free gauge theories. 1, Phys. Rev. D8, 3633-3652 (1973).

[22] S. Eidelman et al., Review of particle physics, Phys. Lett. B592, 1 (2004). 
[23] H. V. Klapdor-Kleingrothaus, A. Dietz, I. V. Krivosheina, and O. Chkvorets, Data acquisition and analysis of the Ge-76 double beta experiment in Gran Sasso 1990-2003, Nucl. Instrum. Meth. A522, 371-406 (2004).

[24] Z. Maki, M. Nakagawa, and S. Sakata, Remarks on the unified model of elementary particles, Prog. Theor. Phys. 28, 870 (1962).

[25] M. Kobayashi and T. Maskawa, CP violation in the renormalizable theory of weak interaction, Prog. Theor. Phys. 49, 652-657 (1973).

[26] L.-L. Chau and W.-Y. Keung, Comments on the parametrization of the Kobayashi-Maskawa matrix, Phys. Rev. Lett. 53, 1802 (1984).

[27] B. Richter, Conventional beams or neutrino factories: The next generation of accelerator-based neutrino experiments, (2000).

[28] J. T. Pantaleone, Constraints on three neutrino mixing from atmospheric and reactor data, Phys. Rev. D49, 2152-2155 (1994).

[29] E. Akhmedov, Three-flavour effects and CP-and T-violation in neutrino oscillations, (2004).

[30] A. D. Sakharov, Violation of CP invariance, C asymmetry, and baryon asymmetry of the universe, Pisma Zh. Eksp. Teor. Fiz. 5, 32-35 (1967).

[31] M. Fukugita and T. Yanagida, Baryogenesis without grand unification, Phys. Lett. B174, 45 (1986).

[32] S. P. Mikheev and A. Y. Smirnov, Resonance enhancement of oscillations in matter and solar neutrino spectroscopy, Sov. J. Nucl. Phys. 42, 913-917 (1985).

[33] L. Wolfenstein, Neutrino oscillations in matter, Phys. Rev. D17, 2369 (1978).

[34] Q. Y. Liu and A. Y. Smirnov, Neutrino mass spectrum with numu to nus oscillations of atmospheric neutrinos, Nucl. Phys. B524, 505-523 (1998). 
[35] J. Davis, Raymond, D. S. Harmer, and K. C. Hoffman, Search for neutrinos from the sun, Phys. Rev. Lett. 20, 1205-1209 (1968).

[36] D. N. Abdurashitov et al., The Russian-American gallium experiment (SAGE) Cr neutrino source measurement, Phys. Rev. Lett. 77, 4708-4711 (1996).

[37] W. Hampel et al., GALLEX solar neutrino observations: Results for GALLEX IV, Phys. Lett. B447, 127-133 (1999).

[38] K. S. Hirata et al., Real time, directional measurement of B-8 solar neutrinos in the Kamiokande-II detector, Phys. Rev. D44, 2241-2260 (1991).

[39] Y. Fukuda et al., Constraints on neutrino oscillation parameters from the measurement of day-night solar neutrino fluxes at Super- Kamiokande, Phys. Rev. Lett. 82, 1810-1814 (1999).

[40] J. Bahcall, Solar Neutrino Viewgraphs, http://www.sns.ias.edu/ jnb/, 2005.

[41] K. Eguchi et al., First results from KamLAND: Evidence for reactor antineutrino disappearance, Phys. Rev. Lett. 90, 021802 (2003).

[42] S. N. Ahmed et al., Measurement of the total active B-8 solar neutrino flux at the Sudbury Neutrino Observatory with enhanced neutral current sensitivity, Phys. Rev. Lett. 92, 181301 (2004).

[43] T. J. Haines et al., Calculation of atmospheric neutrino induced backgrounds in a nucleon decay search, Phys. Rev. Lett. 57, 1986-1989 (1986).

[44] W. A. Mann, Atmospheric neutrinos and the oscillations bonanza, Int. J. Mod. Phys. A15S1, 229-256 (2000).

[45] C. Yanagisawa, Recent results from Super-Kamiokande, in Frontiers in Contemporary Physics 2001, Vanderbilt University, Nashville, Tennesse, USA, 2001.

[46] M. Ambrosio et al., Matter effects in upward-going muons and sterile neutrino oscillations, Phys. Lett. B517, 59-66 (2001). 
[47] T. Kajita, Neutrino physics: experimental status, in Weak Interactions and Neutrinos Workshop 2003 (WIN03), Lake Geneva, Wisconsin, USA, 2003.

[48] E. Aliu et al., Evidence for muon neutrino oscillation in an accelerator-based experiment, Phys. Rev. Lett. 94, 081802 (2005).

[49] C. Bemporad, G. Gratta, and P. Vogel, Reactor-based neutrino oscillation experiments, Rev. Mod. Phys. 74, 297 (2002).

[50] M. Apollonio et al., Search for neutrino oscillations on a long base-line at the CHOOZ nuclear power station, Eur. Phys. J. C27, 331-374 (2003).

[51] K. Okamura, PhD thesis, University of Tokyo, 1999.

[52] A. Aguilar et al., Evidence for neutrino oscillations from the observation of anti-nue appearance in a anti-numu beam, Phys. Rev. D64, 112007 (2001).

[53] S. J. Brice, MiniBooNE, Nucl. Phys. Proc. Suppl. 143, 115-120 (2005).

[54] G. Fogli, Neutrino parameters, in Next Generation of Nucleon Decay and Neutrino Detectors 2005 (NNN05), Aussois, Savoie, France, 2005.

[55] B. Kayser, The neutrino world, in Fermilab Users Meeting '04, Fermilab, Illinois, USA, 2004.

[56] F. Ardellier et al., Letter of intent for double-CHOOZ: A search for the mixing angle theta(13), (2004).

[57] D. S. Ayres et al., NOvA proposal to build a 30-kiloton off-axis detector to study neutrino oscillations in the Fermilab NuMI beamline, (2004).

[58] Y. Itow et al., The JHF-Kamioka neutrino project, (2001), hep-ex/0106019.

[59] W. W. M. Allison et al., Proposal for a long baseline neutrino oscillation experiment from Fermilab to SOUDAN: Update to P822, (1994), FERMILABPROPOSAL-0822-UPD. 
[60] The Soudan 2 Collaboration, Soudan 2: A 1000 ton tracking calorimeter for nucleon decay, Technical report, 1981.

[61] E. Ables et al., P-875: A Long baseline neutrino oscillation experiment at Fermilab, (1995), FERMILAB-PROPOSAL-0875.

[62] The MINOS Collaboration, The MINOS Detectors Technical Design Report, Technical report, 1998, NuMI-GEN-337.

[63] The MINOS Collaboration, The NuMI Beam Technical Design Report, Technical report, 1999, NuMI-346.

[64] B. Becker, R. Piteira, and A. Sousa, Surveying the Construction of Super Module 2, NuMI-GEN-964, 2003.

[65] N. Felt et al., MINOS Far Detector Electronics - User's Manual, NuMI-901, 2002.

[66] J. Hartnell, Measurement of the Calorimetric Energy Scale in MINOS, PhD thesis, St. John's College, Oxford University, 2005.

[67] M. Kordosky, Hadronic Interactions in the MINOS Detectors, $\mathrm{PhD}$ thesis, University of Texas at Austin, 2004.

[68] P. Vahle, Electromagnetic Interactions in the MINOS detectors, PhD thesis, University of Texas at Austin, 2004.

[69] The MINOS Collaboration, Proposal for a Five Year Run Plan for MINOS, NuMI-930, 2003.

[70] M.Diwan et al., A Study of $\nu_{\mu} \rightarrow \nu_{e}$ Sensitivity in MINOS, NuMI-714, 2001.

[71] A. Fasso, A. Ferrari, and P. Sala, Electron photon transport in FLUKA: Status, Prepared for International Conference on Advanced Monte Carlo for Radiation Physics, Particle Transport Simulation and Applications (MC 2000), Lisbon, Portugal, 23-26 Oct 2000. 
[72] A. Fasso, A. Ferrari, P. R. Sala, and J. Ranft, FLUKA: Status and prospects for hadronic applications, Prepared for International Conference on Advanced Monte Carlo for Radiation Physics, Particle Transport Simulation and Applications (MC 2000), Lisbon, Portugal, 23-26 Oct 2000.

[73] H. Gallagher, The NEUGEN neutrino event generator, Nucl. Phys. Proc. Suppl. 112, 188-194 (2002).

[74] R. Hatcher, Active Detector (FLS) Simulation in gminos and reco_minos, NUMI-479, 1999.

[75] R. Hatcher, MINOS Mock Data Challenge, http://home.fnal.gov/ rhatcher/mock_data/mock_data.html, 2004.

[76] B. Rebel and S. Mufson, A Demultiplexing Algorithm for the MINOS Far Detector, NUMI-L-725, 2001.

[77] B. Rebel and S. Mufson, A Demultiplexing Algorithm for Cosmic Rays in the MINOS Far Detector, NUMI-COMP-834, 2002.

[78] R. Lee, Event Reconstruction in the Near Detector, NUMI-COMP-917, 2003.

[79] R. Lee, A Description of the MINOS Reconstruction Framework, NUMICOMP-916, 2003.

[80] R. Lee, User's Guide to the CandEventSR Reconstruction Tree, NUMI-COMP$860,2003$.

[81] R. Hatcher, Numbering Conventions and Coordinate Systems in gminos, NUMI-243, 1997.

[82] P. Blackett and G. Occhialini, Some photographs of the tracks of penetrating radiation, Proc. Roy. Soc. Lond. A139, 699-726 (1933).

[83] H. Bethe and W. Heitler, On the stopping of fast particles and on the creation of positive electrons, Proc. Roy. Soc. Lond. A146, 83-112 (1934). 
[84] C. Leroy and P. Rancoita, Physics of cascading shower generation and propagation in matter: Principles of high-energy, ultrahigh-energy and compensating calorimetry, Rept. Prog. Phys. 63, 505-606 (2000).

[85] H. Bhabha and W. Heitler, Passage of fast electrons and the theory of cosmic showers, Proc. Roy. Soc. Lond. A159, 432-458 (1937).

[86] J. Carlson and J. Oppenheimer, On multiplicative showers, Phys. Rev. 51, 220-231 (1937).

[87] S. Eidelman et al., Review of particle physics, Phys. Lett. B592 (2004).

[88] M. Bosetti et al., Systematic investigation of the electromagnetic energy resolution on sampling frequency using silicon calorimeters, Nucl. Instrum. Meth. A345, 244-249 (1994).

[89] K. Pearson, On lines and planes of closest fit to systems of points in space, Phil. Mag. 2, 559-572 (1901).

[90] R. A. Fisher, The use of multiple measurements in taxonomic problems, Ann. Eugen. 7, 179-188 (1936).

[91] R. Gnanadesikan, Methods for Statistical Data Analysis of Multivariate Observations, Wiley-InterScience, New York, 2nd edition, 1997.

[92] W. R. Dillon and M. Goldstein, Multivariate analysis : methods and applications, Wiley, New York, 1984.

[93] S. Sharma, Applied Multivariate Techniques, Wiley, New York, 1995.

[94] J. Schneps, Multivariate Analysis of Atmospheric Data: Part 1 - The Ratio of Ratios, PDK-690, 1997.

[95] P. Mahalanobis, On the generalized distance in statistics, Proc. Natl. Inst. Science (India) 12, 49-55 (1936). 
[96] T. Hastie, R. Tibshirani, and J. H. Friedman, The Elements of Statistical Learning, Springer, New York, August 2001.

[97] C. Drummond and R. C. Holte, Explicitly representing expected cost: an alternative to ROC representation, in KDD '00: Proceedings of the sixth $A C M$ SIGKDD international conference on Knowledge discovery and data mining, pages 198-207, New York, NY, USA, 2000, ACM Press.

[98] P. Vahle, Nue Analysis Code, http://www.hep.ucl.ac.uk/〜vahle/Meetings/ NueAnalysis, 2004.

[99] H. Gallagher and M. Goodman, Neutrino Cross Sections, NUMI-112, 1995.

[100] Z. Koba, H. B. Nielsen, and P. Olesen, Scaling of multiplicity distributions in high-energy hadron collisions, Nucl. Phys. B40, 317-334 (1972).

[101] R. P. Feynman, M. Kislinger, and F. Ravndal, Current matrix elements from a relativistic quark model, Phys. Rev. D3, 2706-2732 (1971).

[102] D. Rein and L. M. Sehgal, Neutrino excitation of baryon resonances and single pion production, Ann. Phys. 133, 79 (1981).

[103] D. Groom et al., Review of Particle Physics, The European Physical Journal C15 (2000).

[104] W. H. Press, B. P. Flannery, S. A. Teukolsky, and W. T. Vetterling, Numerical Recipes: The Art of Scientific Computing, Cambridge University Press, Cambridge (UK) and New York, 2nd edition, 1992.

[105] R. Raja, The main injector particle production experiment (MIPP) at Fermilab, J. Phys. Conf. Ser. 9, 303-308 (2005).

[106] D. Drakoulakos et al., Proposal to perform a high-statistics neutrino scattering experiment using a fine-grained detector in the NuMI beam, (2004).

[107] T. Yang and S. Wojcicki, Near detector data/mc comparison for nue analysis, in preparation, 2005. 